\title{
Applying Funds of Knowledge in a New Zealand High School: The Emergence of Team-based Collaboration as an Approach
}

\author{
Linda Mary Hogg
}

\author{
A thesis \\ submitted to Victoria University of Wellington \\ in fulfilment of the requirements for the degree of \\ Doctor of Philosophy in Education
}

Victoria University of Wellington

November 2013 


\section{Abstract}

Underachievement by Māori and Pasifika students in New Zealand schools evidences the need for more effective teacher practices to support minoritized students' learning, in the interests of social justice. Learning about students' funds of knowledge (FoK) allows teachers to offer relevant learning experiences, achieving a fuller socio-constructivist approach to teaching and learning (González, Moll, \& Amanti, 2005). This study explores the relevance of the FoK concept in the New Zealand context, and its application at high school level.

For the purposes of this qualitative study, participants $(n=21)$ from a high school community were organized into five teams; three teams included a teacher, students, and their parents, and two included a teacher and students. Each team negotiated, implemented, and evaluated strategies for the teacher to learn about students' FoK.

Findings highlight the dual layer of benefits arising from participation in collaborative teams and teacher inquiry into students' FoK. Each team formed a temporary system which transcended school norms and values, allowed members to interact in new ways, and provided a safe space for exploration of the application of FoK. A conceptual map outlining elements of the team-based collaboration (TBC) approach identifies factors related to purpose, participants, and process which generated each team's culture. Complex interplay of contextual elements influenced teams' milieu, decision-making, and valued outcomes.

Valued outcomes included: identification of diverse FoK held by Māori and Pasifika students; development of closer teacher-student relationships and increased mutual commitment; greater evidence of effective learning behaviors in class; and pedagogical applications that were congruent with existing teaching programs. Barriers to valued outcomes suggest areas for participant training; these included the matching of strategy choice to participants to illuminate FoK, traditional teacherstudent dynamics, and time and timing. Implications for teacher practice and future research are identified. 


\section{Acknowledgements}

I learned that courage was not the absence of fear, but the triumph over it.

- Nelson Mandela

This study has been a collaborative effort. I am very grateful to all my participants, especially the students, parents and teachers who made up the teams. I owe all the participants heartfelt thanks for allowing me to work with them, and for being so generous with themselves and their time.

Dr Liz Jones and Emeritus Professor Cedric Hall, my supervisors, have provided calm constancy, meticulous care, excellent questions, and wise guidance; I owe a huge debt of gratitude for their support. I'm also grateful for conversations with my cohort group and other colleagues, and for Susan Kaiser's expert help in preparing the final manuscript. Thank you to my esteemed examiners, I greatly value your perspectives and critical feedback. It has been a privilege to undertake this work with everyone who has been involved.

Thank you to the precious people who have given me love, support, and sustenance throughout this project. I would like to single out two special individuals. Thank you Huia for your co-operation and patience as I worked towards my goal. You generously allowed me to immerse myself in this study that is so important to me. Thank you Eddy, your love, faith, and care give me strength and feed my heart. Your experience, insight and inquiring mind enhance my perspective. Lastly, your beautiful graphics are an integral part of this thesis.

Reflecting on this work highlighted funds of knowledge from my forebears. I acknowledge their courage to forge new paths, and resilience in the face of challenges, which I drew on in this work.

This thesis is dedicated to Huia:

Follow your dreams with passion, commitment, and courage,

Learn and express your true identity as a huia,

And you will attain your full beauty and soar above the clouds 


\section{Table of Contents}

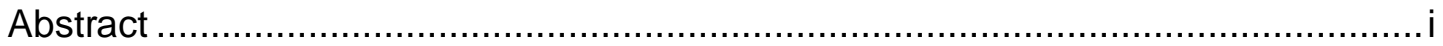

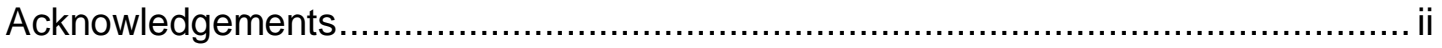

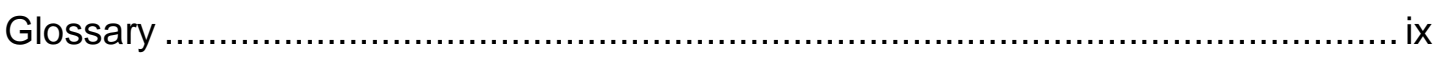

Chapter 1: Identifying the Research Focus........................................................

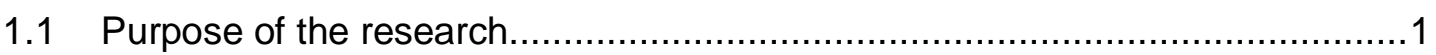

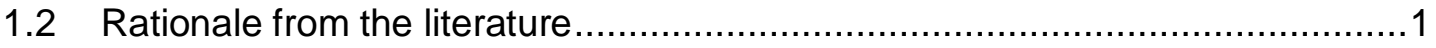

1.2.1 Ethnic differences in schooling experience and outcomes.................1

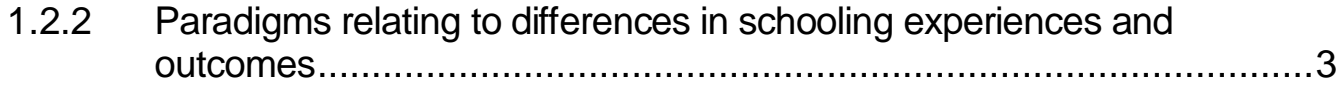

1.2.3 Education for a multicultural society ...........................................

1.2.4 Funds of knowledge (FoK) .................................................. 4

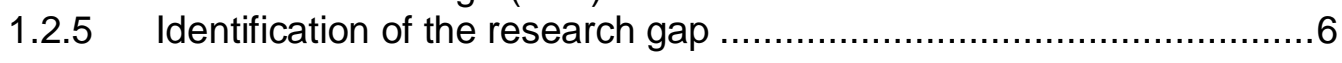

1.3 Personal relevance of the topic ...............................................................

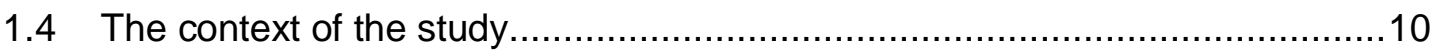

1.4.1 National educational policies in NZ .........................................10

1.4.2 Kereru High School ..................................................................... 12

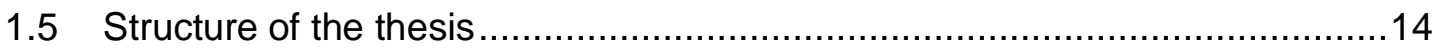

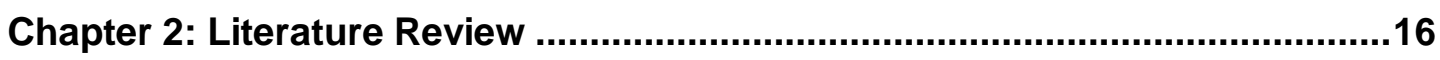

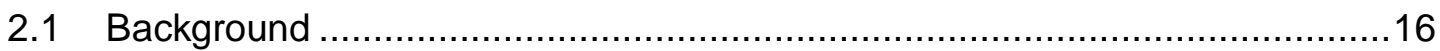

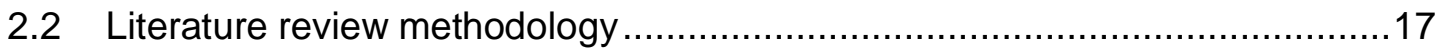

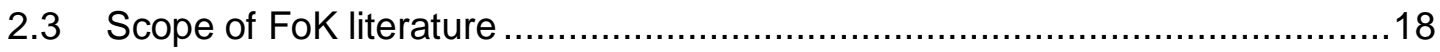

2.4 Definitions of funds of knowledge ...................................................... 19

2.4.1 FoK: A contested term ....................................................... 19

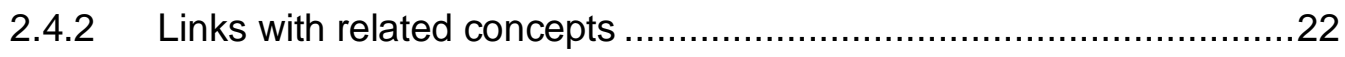

2.5 Theoretical frameworks for funds of knowledge scholarship ........................25

2.6 Approaches to identify students' funds of knowledge .................................26

2.7 Valued outcomes arising when teachers learn about students' FoK...................33

2.7.1 Illumination of rich funds of knowledge..........................................3

2.7.2 Changes in teacher perceptions......................................................

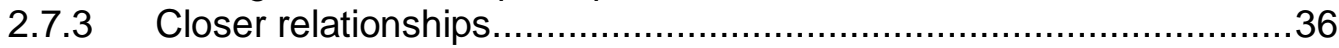

2.7.4 Applying FoK to classroom pedagogy .......................................37

2.8 Limitations of current research ..............................................................43

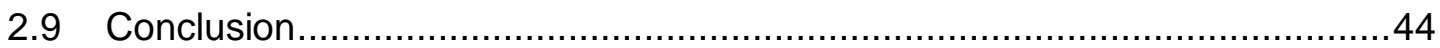

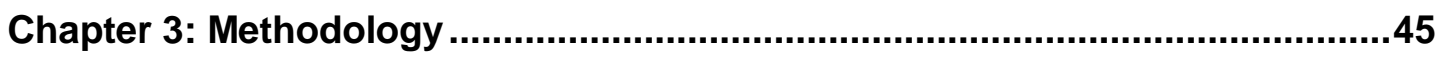

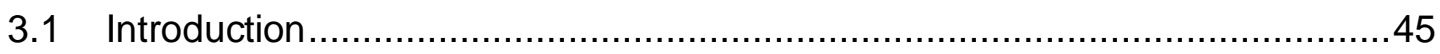

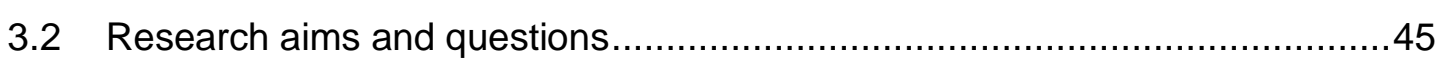

3.3 Operational definitions .................................................................... 47 


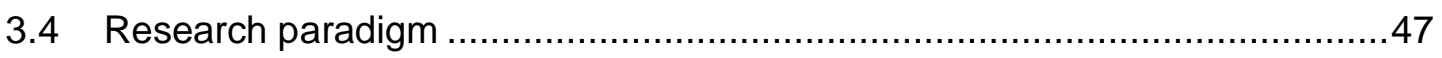

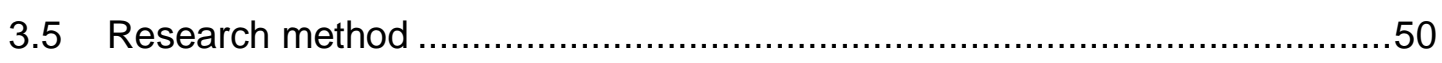

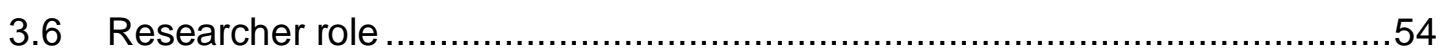

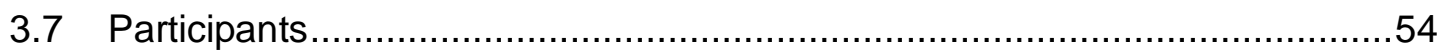

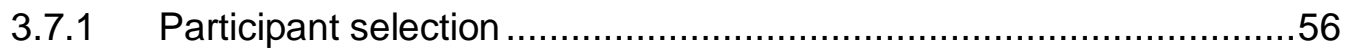

3.7.2 Participant recruitment ........................................................ 57

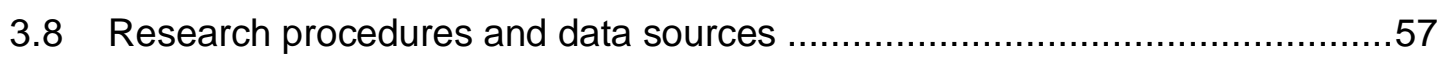

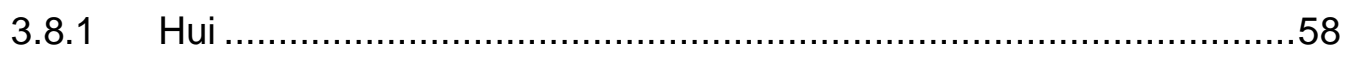

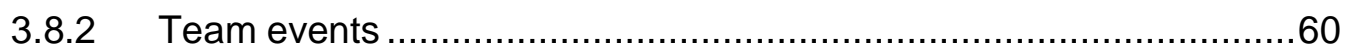

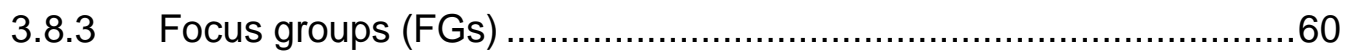

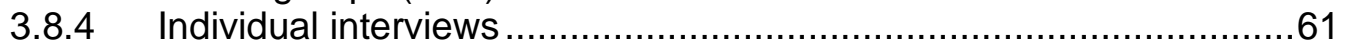

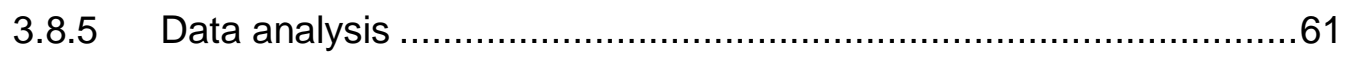

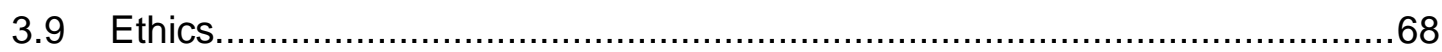

3.9.1 Voluntary informed consent......................................................68

3.9.2 Non-maleficence and beneficence ...........................................69

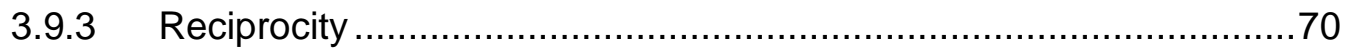

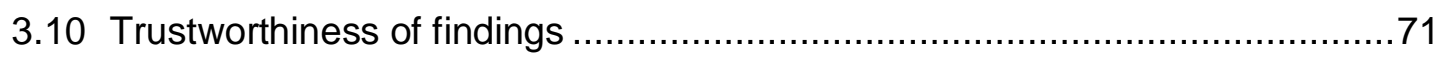

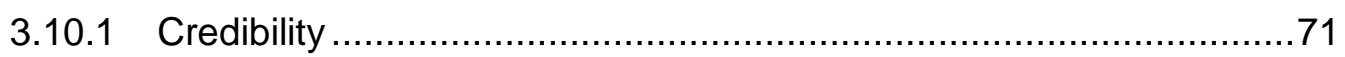

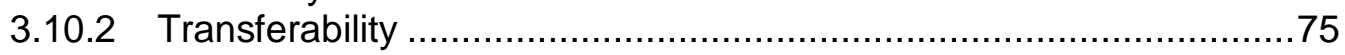

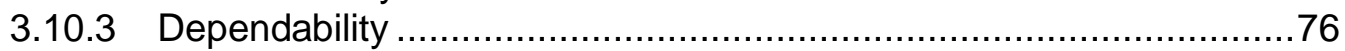

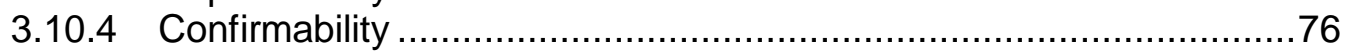

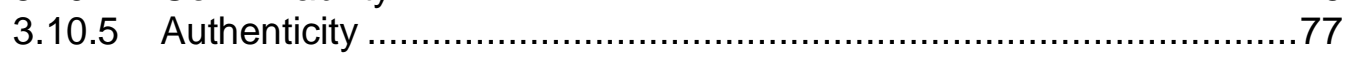

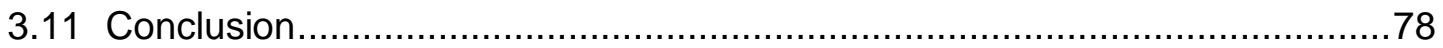

Chapter 4: Team A Findings ......................................................................79

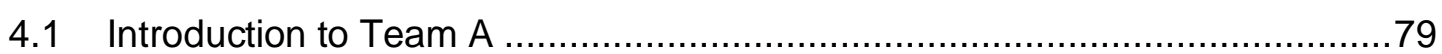

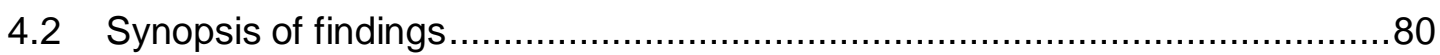

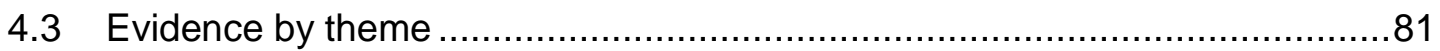

4.3.1 Preferred approaches for teacher to learn about students' FoK........81

4.3.2 Enablers that supported valued outcomes in Team A ....................81

4.3.3 Barriers to valued outcomes in Team A........................................ 86

4.3.4 Valued outcomes achieved ......................................................91

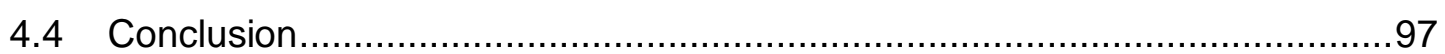

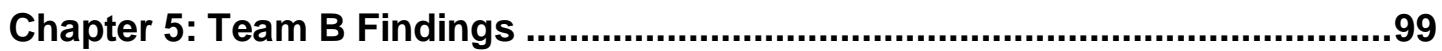

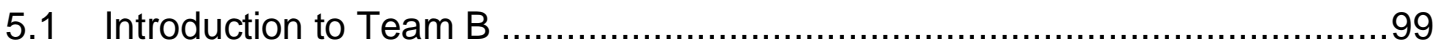

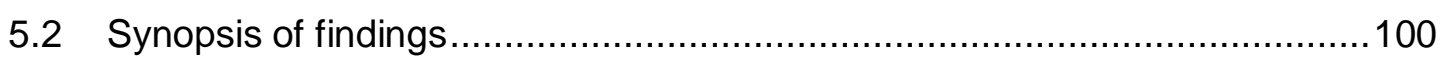

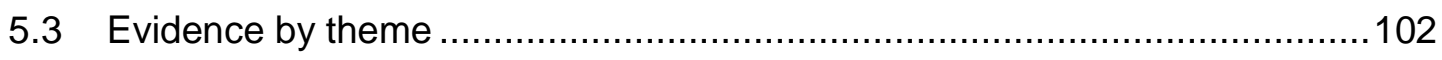

5.3.1 Preferred approaches for teacher to learn about students' FoK ......102

5.3.2 Enablers that supported valued outcomes in Team B ...................102

5.3.3 Barriers to valued outcomes in Team B.....................................112

5.3.4 Valued outcomes achieved .................................................... 114

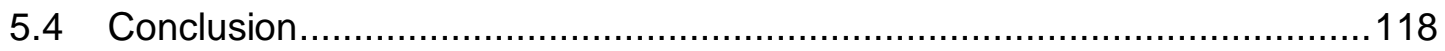


Chapter 6: Team C Findings

6.1 Introduction to Team C ................................................................. 120

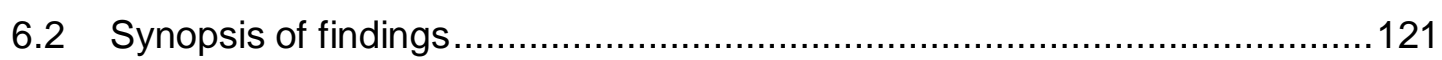

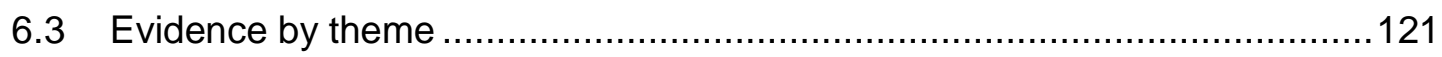

6.3.1 Preferred approaches for teacher to learn about students' FoK......121

6.3.2 Enablers that supported valued outcomes in Team C ....................122

6.3.3 Barriers to valued outcomes in Team C .....................................133

6.3.4 Valued outcomes achieved ................................................... 135

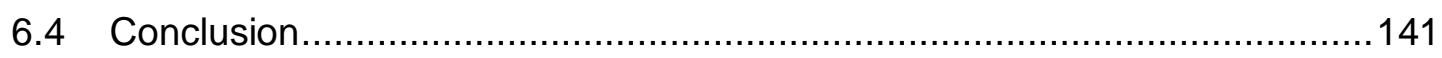

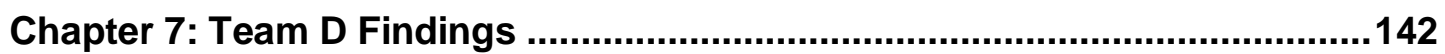

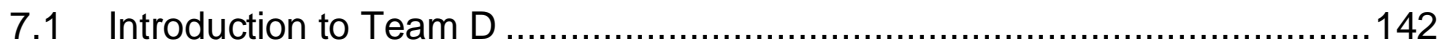

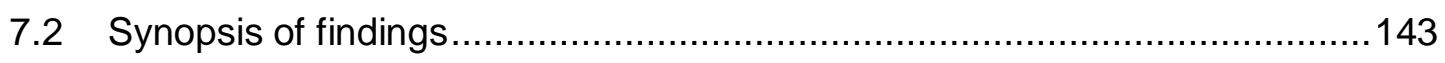

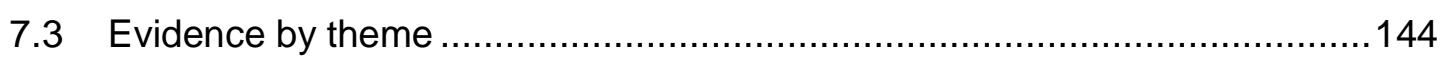

7.3.1 Preferred approaches for teacher to learn about students' FoK...... 144

7.3.2 Barriers to valued outcomes in Team D ..................................... 144

7.3.3 Valued outcomes achieved .................................................. 159

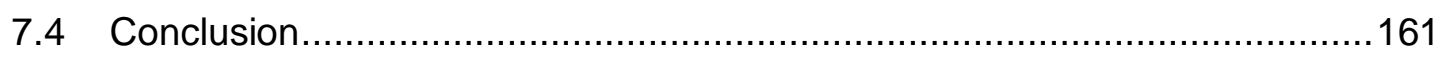

Chapter 8: Team E Findings...................................................................... 162

8.1 Introduction to Team E ................................................................... 162

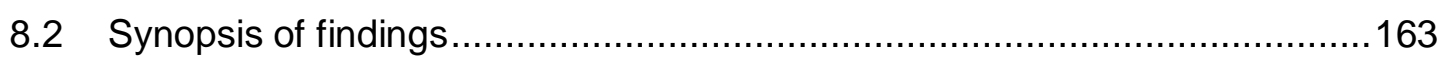

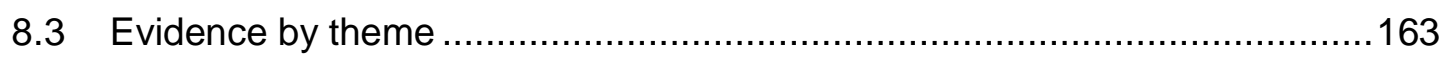

8.3.1 Preferred approaches for teacher to learn about students' FoK......163

8.3.2 Enablers that supported valued outcomes in Team E ...................164

8.3.3 Barriers to valued outcomes in Team E.....................................173

8.3.4 Valued outcomes achieved ................................................. 179

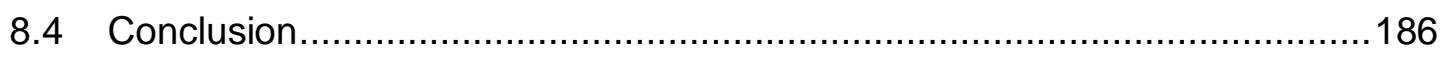

Chapter 9: Cross-team Findings ................................................................187

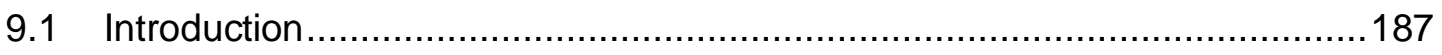

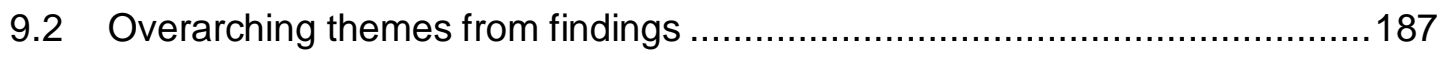

9.2.1 Team structure and process....................................................187

9.2.2 Strategies for learning about students' FoK .................................189

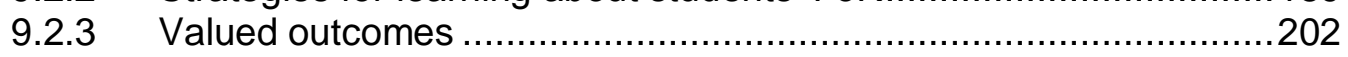

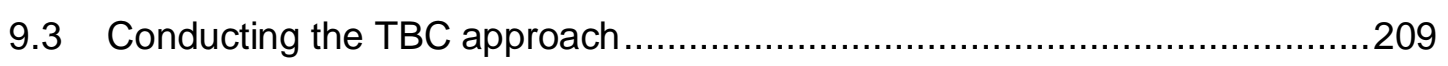

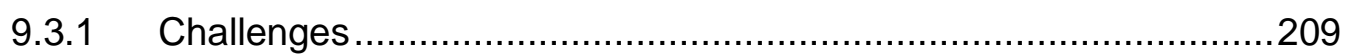

9.3.2 Supportive factors ........................................................... 217

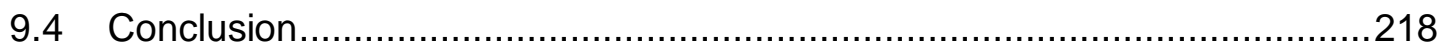


Chapter 10: Discussion

10.1 Purpose and contribution of the study ..............................................219

10.2 Outcomes from teachers learning about Māori and Pasifika students' FoK ....... 220

10.2.1 Illumination of rich FoK......................................................220

10.2.2 Teacher-student relationships and effective learning behaviors .....222

10.2.3 Applying FoK to pedagogical practice .......................................224

10.3 Outcomes from the team-based collaboration approach (TBC) ...................226

10.3.1 Elements of TBC ................................................................226

10.3.2 Teams were systems that transcended school and family cultures ....229

10.4 Influence of other contextual factors on findings ......................................234

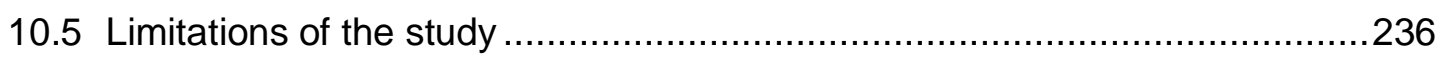

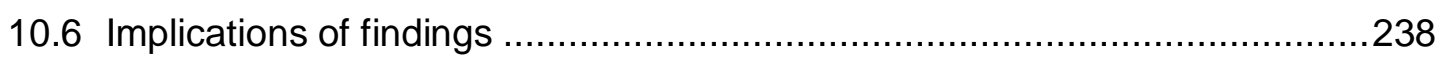

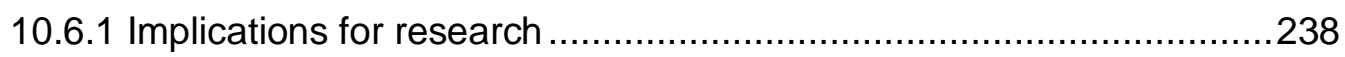

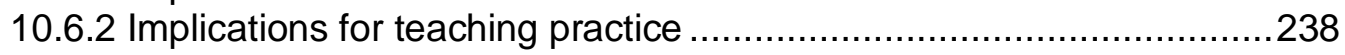

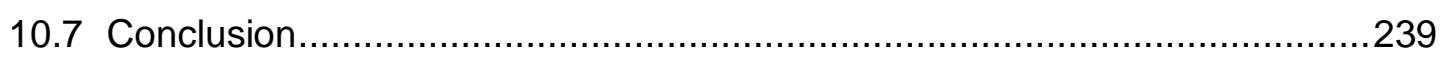

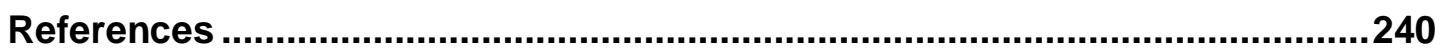

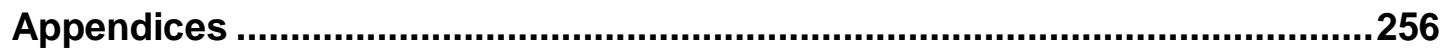

Appendix A: Confirmation of ethical approval for the research ..........................257

Appendix B: Completion of research events by participants, by team ..................258

Appendix C: Broad themes in findings emerging from data analysis ....................260 


\section{List of Tables}

Table 2.1: Related concepts 23

Table 2.2: Methodologies employed or described to identify students' funds of knowledge

Table 2.3: Classifications of pedagogical applications of FoK (Rodriguez, 2013).....37

Table 3.1: Key elements of this case study................................................50

Table 3.2: Overview of demographic profile of participants, by team .....................55

Table 3.3: Overview of demographic profile of participants, by group....................55

Table 3.4: Data collection related to research questions....................................58

Table 3.5: Node summary table (extract) .......................................................65

Table 3.6: Time spent in the field............................................................ 72

Table 4.1: Examples of positive inclusive actions in Team A .............................82

Table 4.2: Attendance, absence and lateness in Team A ....................................88

Table 5.1: Examples of ways that Kate talked to students ................................. 106

Table 6.1: Contributions of Team C participants in team hui..............................123

Table 6.2: Ways that Georgia positioned herself as a learner..............................127

Table 6.3: Examples of students' FoK revealed by Team C strategies ................. 135

Table 8.1: Acknowledgement of students' FoK by Tom ................................... 167

Table 8.2: Examples of FoK and ways they were shown .................................. 171

Table 8.3: Dates of strategy decisions, planned and actual implementation ........178

Table 9.1: Teams' approach to achieving inclusivity ........................................ 188

Table 9.2: Strategies agreed and implemented by teams ................................. 190

Table 9.3: Selected features of chosen strategies ......................................... 191

Table 9.4: Students' assessment of relationship with teacher before and after fieldwork .................................................................... 205

Table 9.5: Application of FoK to classroom pedagogy by teams ........................209

Table 9.6: Features of participant observation related to this study .....................211

Table 9.7: Researcher contributions in team hui.............................................213

Table 10.1: Examples of students' FoK .......................................................220 


\section{List of Figures}

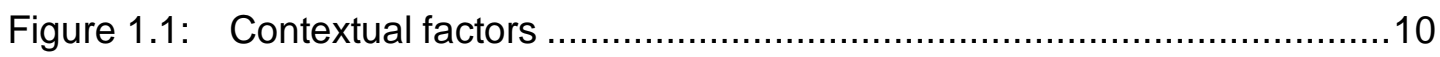

Figure 2.1: Relationship between participatory learning theories, processual approaches to culture, and funds of knowledge..............................21

Figure 2.2: Conceptual bases identified in funds of knowledge texts ...................25

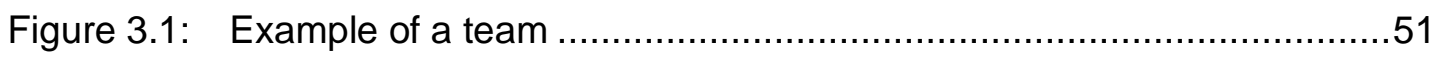

Figure 3.2: Cyclical process undertaken by teams .........................................52

Figure 3.3: Graphic overview: Themes from Team D .....................................66

Figure 4.1: Team A participants at start and end of study ...............................79

Figure 5.1: Team B participants ........................................................... 100

Figure 5.2: Elements of whanaungatanga .................................................. 109

Figure 6.1: Team C participants .......................................................... 120

Figure 7.1: Team D participants at start and end of study ................................ 142

Figure 8.1: Team E participants .............................................................. 162

Figure 8.2: Conversation pattern in Team E ............................................. 165

Figure 10.1: Conceptual map: Elements of the TBC approach.............................227

Figure 10.2 The location and nature of the temporary overlapping boundary ......231

Figure 10.3 Congruence between internal and external contextual elements:

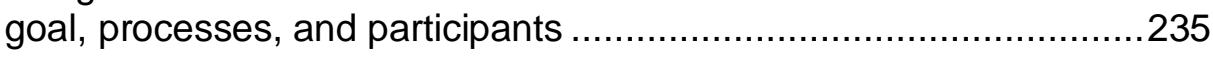




\section{Glossary}

\section{Māori or New \\ Zealand word}

ākonga

dean

decile

E Tipu E Rea

He Kākano

Hui

Integrated Studies

Ka Hikitia

Kapa haka

Kereru

Manaakitanga

Māori

Māoritanga

Marae

NCEA (National

Certificate of

Educational

Achievement)

\section{Meaning}

Learner

Pastoral care role, involving care of students in a year level

Decile rating is an indicator of socio-economic status of communities. In New Zealand, each school is assigned a decile rating indicative of the extent to which it draws its students from low socio-economic communities. The rating scale sorts approximately $10 \%$ of schools into each of ten deciles: Decile 10 schools draw the least from low socio-economic (SES) communities, whereas decile 1 schools include the largest proportions of students from low-SES communities (Ministry of Education, n.d.).

Kereru High School's local version of Te Kotahitanga. This name is a pseudonym.

A TPL initiative with a focus on management systems and structures, to improve their alignment with features of school leadership that are effective for Māori students.

Meeting. The Māori term hui is used for many research events, to show respect for the Māori participants.

Some classes at Kereru High School were engaged in an Integrated Studies program. This program created a framework whereby common thematic units of study were applied in students' English, Social Studies, Science, and Mathematics classes. Therefore, learning in four subject areas was linked, to enhance students' experience of congruence across subject disciplines.

To step up, to lift up, to lengthen one's stride; also the name of the Māori Education Strategy, 2008-2012

Māori performing arts

Wood pigeon native to New Zealand

Manaakitanga is a Māori term which encompasses ideas related to respect, hospitality, nurturing, and kindness.

Indigenous peoples of New Zealand

Māori ways of doing things

Sacred Māori building complex for public ceremonial use, including a meeting room (wharenui), kitchen and dining area (wharekai) and other spaces (Adds, Hall, Higgins, \& Higgins, 2011).

New Zealand academic qualification. NCEA qualifications relevant to each cohort group of school students were: Year 11: NCEA Level One; Year 12: NCEA Level Two; Year 13: NCEA Level Three. To earn credits for NCEA, students complete internal and external assessment tasks.

Pākehā
New Zealander of predominantly European descent, Caucasian 
Māori or New

Papa

(Papatuanuku)

Pasifika

Pepeha

Rangi

(Ranginui)

Te Kotahitanga

Te reo Māori

Tikanga Māori

TPL

Whaea

Whakataukī

Whānau

Whakapapa

Years 9-13

\section{Meaning}

Papa is a Māori god, the Earth mother, who with Rangi has an important role in Māori creation mythology.

A general term which refers to peoples from all Pacific nations, although these peoples are ethnically and culturally diverse. Some Pasifika peoples encompassed by this term include Samoan and Cook Island Māori.

Pepeha is a Māori term which means an introduction of oneself.

Rangi is a Māori god, the sky father, who with Papa has an important role in Māori creation mythology.

A large scale research and professional development project which began in New Zealand in 2001, with the aim of improving mainstream schooling for Māori The project has three strands: gathering student narratives and other data related to Māori students' schooling experience; using student narratives and other literature to develop a description of effective practice; and using student narratives as a tool for TPL.

Māori language

Māori shared practices and principles

Teacher Professional Learning

Mother; used to address a woman enacting this role

Māori proverb

Extended family or group of people with a strong bond

Whakapapa is a Māori term for genealogy, expression of identity through the establishment of links. This is a very important concept for Māori, who define themselves by their whakapapa.

Cohort levels for New Zealand high school students. Equivalencies with U. S. grades are:

Year $9=$ Grade 8

Year $10=$ Grade 9

Year $11=$ Grade 10

Year $12=$ Grade 11

Year $13=$ Grade 12 


\section{Chapter 1: Identifying the Research Focus}

This chapter sets the scene for the research. Firstly the rationale for the study is presented, drawing on literature and factors that attracted me personally to the field and research questions. The research was conducted in a New Zealand (NZ) secondary school; therefore, in the second half of the chapter NZ's national education environment and school setting are described. Lastly the overall structure of the thesis is explained.

\subsection{Purpose of the research}

The primary purpose of the research was to investigate perceptions of valued outcomes arising from teacher inquiry to identify ethnic minority students' funds of knowledge (FoK) within the context of a NZ secondary school.

\subsection{Rationale from the literature}

\subsubsection{Ethnic differences in schooling experience and outcomes}

In 2010, Penetito noted that "the achievement gap between Māori and non-Māori has been an embarrassment to governments for at least 30 years" (p. 259), highlighting New Zealand schooling's longstanding failure to fulfil the potential of Māori students. Reflecting on her personal experience as a Māori high school student in the 1990s, Grootveld (2013) states:

One of the striking memories I have about my secondary education was the number of Māori and Pasifika friends who dropped out or were kicked out of school along the way. I knew then that something just didn't add up. From a class of 30-something Māori students in Year 9, to only five of us left in Year 13... This was not about naughty Māori kids with behavioral issues and whānau who didn't care. This was about a system that excluded students and whānau, many (not all) teachers with deficit attitudes, an under-resourced, low-decile school, a curriculum based on Western knowledge, a lack of positive Māori academic role models, and students who were bored and could not see the value and relevance of academic learning. (p. 27)

Recent educational statistics demonstrate the persistence of systemic ethnic educational disparities in schooling experience. For instance, in 2008, NZ achievement data for Year 11 secondary school students relating to the attainment of the New Zealand Certificate of Educational Achievement (NCEA) Level One show considerable differences between ethnic groups. NCEA Level One, the basic school 
qualification, was achieved by $48 \%$ of Pasifika ${ }^{1}, 53.5 \%$ of Māori, $79.6 \%$ of Pākehā, and $74.5 \%$ of Asian students. This pattern of around $20 \%$ differential in the success rate of Māori and Pākehā students is repeated for Year 12 and 13 students entered for NCEA Levels Two and Three (NZQA, 2010). These achievement gaps have reduced since 2002, when $35 \%$ of Year 11 Māori and $30 \%$ of Year 11 Pasifika students achieved NCEA Level One (Mallard, 2003). However, the relative underachievement of Māori and Pasifika students remains a serious concern.

Māori and Pasifika students dominate discipline statistics in NZ, as measured by stand-downs $^{2}$ and suspensions ${ }^{3}$. Forty-seven per cent of students suspended in 2000 were Māori, who comprised 21\% of the total school population (Ministry of Education, 2005). In 2006, $21.7 \%$ of students were Māori, but with a stand-down rate of 56 per 1000 and a suspension rate of 15 per thousand, Māori were the ethnic group most likely to be stood-down or suspended from school (Ministry of Education, 2007a). Pasifika students are also over-represented in suspension statistics. Although in 2006 Pasifika students comprised $9.1 \%$ of school students, their stand-down rate was 42 per 1000 students, and their suspension rate was 10 per 1000 students (Ministry of Education, 2007a). These statistics echo similar ethnic disparity in American discipline statistics, where recent parent reports indicate $19.6 \%$ of Black students were suspended, compared to less than $9 \%$ of Whites and fewer than 7\% of Asian students (KewelRamani, Gilbertson, Fox, \& Provasnik, 2007). Moreover, differences in the ways that students from the various ethnic groups are disciplined, together with links between disciplinary procedures and academic achievement, highlight concerning causes and effects of school discipline practices (Gregory, Skiba, \& Noguera, 2010).

Alternative education (AE) sites in NZ provide schooling for a very small minority of Year 9-11 students alienated from mainstream education, due to either multiple expulsions, behavioral problems, or other issues (Ministry of Education, 2002b). In 2000 , Māori comprised $25 \%$ of secondary school students and $78.3 \%$ of students in $A E$ (Denny, Clark, \& Watson, 2004). Although students in $A E$ reported their $A E$ environments were supportive and nurturing (Denny et al., 2004), it is concerning that Māori students are more likely to be alienated from mainstream schooling. Given the strong directive embedded in government policies regarding national goals of inclusivity and social equity, and the government's acknowledgement of

1 In this thesis Pasifika is used as an umbrella term, to encompass diverse peoples from South Pacific ancestry, such as Samoans and Cook Islanders.

2 Stand-downs, which disallow students from attending school for up to five days, are decided by school principals in the event of serious breaches of school rules

3 Suspensions are initially decided by principals for very serious breaches of rules; they may result in the suspended student being excluded from a school. 
underperformance of the education system for Māori (MoE, 2010), these statistics present an urgent challenge to NZ schools and teacher educators (Hogg, 2008).

\subsubsection{Paradigms relating to differences in schooling experiences and outcomes}

Various theories have been advanced to explain ethnic and racial disparities in student achievement. Relevant factors include students' socio-economic status (Harker \& Nash, 1990; Nicholson, 2000), student culture (Belgrave \& Allison, 2006), teacher expectations (Good \& Brophy, 1970; Rosenthal \& Jacobson, 1966; Timperley \& Robinson, 2002), teacher-student relationships (Bishop \& Berryman, 2006; Hawk, Cowley, Hill, \& Sutherland, 2002), monocultural schools (Bishop \& Glynn, 1999), teacher quality (Darling-Hammond \& Bransford, 2005; Hardy, 2006), teachers' pedagogical practice (Alton-Lee, 2003), teacher competence and use of culturally responsive teaching practice (Banks, 2007; Baskerville \& Bondy, 2010), and culturally responsive classroom management (Savage, 2010).

One suggested cause of underachievement by some ethnic groups is deficit theorizing by teachers (Bishop \& Berryman, 2006; González, 1995; Irvine \& York, 1993; Valencia, 2010). A teacher's deficit mindset may be hidden from the holder, due to unconsciously held attitudes and beliefs, and lack of understanding of how these may create obstacles to student achievement (Aguilar \& Pohan, 1996). Bishop (2005) argues that in NZ, as a result of the dominant colonialist discourse, deficit thinking is an entrenched mindset. By this Bishop means that deficit thinking is intricately linked with prevailing notions of schools as a tool of power and control with a missionary civilizing role, to redress perceived inferiority in indigenous Māori. Bishop, Berryman, Tiakiwai, and Richardson (2003) report that most ideas expressed by NZ teachers about Māori students state that Māori children themselves, their homes, and life outside school are to blame for their poor educational achievement; they also found that many teachers expressed frustration regarding Māori students' low achievement levels. This suggests that, simultaneously, teachers want to support Māori students and are constrained by their deficit thinking.

\subsubsection{Education for a multicultural society}

As for other international settings, the NZ population is becoming increasingly ethnically diverse, while teachers remain relatively homogenous. Thus, disparity is growing between teaching professionals - mainly middle class white females - and students - projected to be 35\% Polynesian by 2021 (Ministry of Education, 2002a). Teachers can work effectively with students from cultures other than their own (Hawk, et al., 2002) when they can relate to them, and support their identity and 
learning as cultural beings (Bishop et al., 2003; Hawk, et al., 2002). When teachers appreciate that they themselves are cultural beings, as are all individuals (Delpit, 1995), self-awareness of unconsciously held cultural perspectives, values, and practices can develop (J. E. King, 2004).

Children from both working class and middle class backgrounds have access to language-rich environments (Heath, 1983). Outside school, minority ethnicity children engage with rich literacy resources and experiences (Andrews \& Yee, 2006; Smythe \& Toohey, 2009). However, in school, teachers tend to recognize and draw on knowledge and experiences of white middle class children much more frequently. The richness of ethnic minority children's lifeworld experience tends to far exceed that of their school experience (Andrews \& Yee, 2006). Therefore, many "disadvantaged" students are actually disadvantaged by a fundamental misalignment between their own FoK and that of the teacher (Baker, Street, \& Tomlin, 2001; Irvine, 2003; Rosebery, McIntyre, \& González, 2001; Vélez-Ibáñez \& Greenberg, 1992). This is not a neutral situation, since students' and teachers' FoK may be in direct conflict with one another (Ballenger, 1999; González, 1995; Michaels, 1985; Tharp \& Gallimore, 1993; Vélez-lbáñez \& Greenberg, 1992; Warren \& Rosebery, 1996). Potential consequences may include learning and/or relationship problems between teacher and student, due to underlying differences or misunderstandings of "our ways of being in the world" (Gee, 1996, p. viii). Ethnic minority students who succeed report achievement involved loss of cultural identity (Bishop \& Glynn, 1999; Fordham \& Ogbu, 1986; Penetito, 2010).

Working class and some ethnic minority students (such as African American and Latino $^{4}$ in USA, Afro-Caribbean in Britain and France, and Māori in NZ) may be described as minoritized. Rather than relating to their numerical status in a society, this term describes students who "have been ascribed characteristics of a minority and are treated as if their position and perspective is of less worth" (Sleeter, 2011, p. 1). This term is used throughout the thesis, because it encompasses a range of student groups for whom there are serious social justice concerns.

\subsubsection{Funds of knowledge (FoK)}

When considered alongside changing demographic structures, a range of research findings highlight the urgent need to identify strategies to enhance connections between dominant-culture teachers and students from non-dominant cultural groups.

4 In this thesis Latino is used as an umbrella term, to encompass diverse peoples from Latin American ancestry, such as Mexicans and Puerto Ricans. 
The FoK concept (Moll, Amanti, Neff, \& González, 1992; Moll \& Greenberg 1990; Moll et al., 1990) offers such a possibility, presenting an assertive rebuttal of the prevalent deficit theorizing mindset. It offers a mechanism to reframe negative, fatalistic views of minoritized students. By challenging stereotyped thinking and low expectations for these groups, Moll et al. (1990) argue that teacher engagement with the FoK concept could allow the eventual possibility of improved pedagogical practices, and greater student achievement across diverse students.

FoK has been defined by leading scholars (Moll et al., 1992, p. 134) as "historically accumulated and culturally developed bodies of knowledge and skills essential for household or individual functioning and wellbeing" 5 . A range of studies in diverse fields, including literacy, cultural geography, and youth cultures provide evidence of the diversity of FoK in different contexts (Thomson \& Hall, 2008). Some examples of students' FoK are desert gardening (Kahn \& Civil, 2001), bilingualism (Dworin, 2006; Moll, 1992), musical expertise (Conant, Rosebery, Warren, \& Hudicourt-Barnes, 2001) and specific language vernacular, such as signifying (Lee, 2001). Zipin (2009) noted students' FoK may be "dark" (p. 320), for instance, knowledge about bullying, alcoholism, discrimination, or other challenging issues. FoK held by Latino teenagers from low income, working class families in Detroit, Michigan, USA came from "homes, peer groups and other systems and networks of relationships" (Moje et al., 2004, p. 38), including popular culture. Andrews and Yee (2006) underscore FoK's essentially personalized quality, arguing FoK are dynamic, like students and their families.

If teachers do not strategically seek information about students' FoK, then these remain hidden and may subvert new learning (Moje et al., 2004). Patterson and Baldwin (2001) report FoK research "brought us face to face with our ignorance, and our arrogance" (p. 127). These findings highlight the importance of teachers learning about their students, to minimize problems or missed opportunities arising from mismatch of formal learning experiences and students' FoK.

To develop knowledge and skills to successfully support culturally diverse students, teachers need to learn about students as individuals. Specifically, knowing students' FoK allows teachers to offer relevant learning experiences, achieving a fuller socioconstructivist approach to teaching and learning (González et al., 2005). How can this be done? Reliance on information about characteristics and pedagogies of cultural groups - characterized by generalized information from secondary sources - has pitfalls; for example, its effectiveness is constrained by the degree to which

5 Section 2.4.1 discusses commonalities and differences in definitions of FoK employed by different scholars, and identifies and justifies the definition utilized in the current study. 
cultural groups themselves are homogenous and static (González, 1995). Also, some individual differences include behavior and degree of identification with the cultural group (Hansen, 1992). In our shrinking world, approximately 200 million people live outside their birth country (Vertovec, 2009), and "translocal, transnational, and transborder communities" (González, Andrade, Civil, \& Moll, 2001 , p. 116) are growing. Change from migration and technological advances continually create new and mixed practices. "Students increasingly draw from an intercultural and hybrid knowledge base, appropriating multiple cultural systems, as youth culture permeates greater and greater spheres" (González, 2005, p. 37). Thus sociocultural influences and other aspects of identity make each individual unique (Grant \& Sleeter, 2007), raising questions about meaningful frameworks for teachers to know their students.

FoK offers a conceptual framework for informing effective practice for minoritized students. It is centered on the principle that the best way to learn about lives and backgrounds is through a focus on learning about "what people do and what they say about what they do" (González, 2005, p. 40). The potential of this approach lies in its ability to identify what is - rather than what is missing, and to engage with individuals - rather than assumptions and stereotypes. It thus brings a post-modern perspective (González, 1995) to multicultural education. With knowledge of students' FoK, teachers can draw on students' experiences and priorities, validate their knowledge and life values, and scaffold learning from the familiar. This means that when teachers recognize and value personalized communities of practice (Lave \& Wenger, 1991) and FoK students have gained from them, then these Discourses (Gee, 1999) can support students' apprenticeship into academic fields of Discourse.

\subsubsection{Identification of the research gap}

The use of the FoK concept has been very positively received, particularly by volunteer teachers and Latino families in Tucson, Arizona, USA (González et al., 2005), but there is a relative paucity of knowledge of its application at high school level, particularly in NZ.

This study explores use of the FoK concept in the context of a NZ high school, focusing on seeking evidence of its value. The research question and sub-questions as stated provide a clear unit of analysis (valued outcomes) and enable investigation of participants' hoped-for valued outcomes, and actual valued outcomes achieved. Preferred strategies for teachers to learn about students' FoK are identified, as defined by collaboratively selected strategies by teacher, student, and parent participants. Issues that enhanced or constrained strategy effectiveness are described. Thus, the research question was designed to generate findings related to 
preferred approaches for identifying students' FoK and the perceived worth of this endeavor as identified by participants.

\title{
1.3 Personal relevance of the topic
}

Discussing researcher subjectivity, Fine and Weis (2002) wrote:

\begin{abstract}
Our obligation is to come clean "at the hyphen", meaning that we interrogate in our writings who we are as we co-produce the narratives we presume to collect. It is now acknowledged that we, as critical ethnographers, have a responsibility to talk about our own identities, why we interrogate what we do, what we choose not to report, on whom we train our scholarly gaze, who is protected and not protected as we do our work. (p. 284)
\end{abstract}

This section is a personal statement of key aspects of my identity, shaped by sociocultural contexts within my personal and professional life thus far. I begin by describing some elements of my personal history that led me to this study and influenced my knowledge and beliefs as the researcher. Secondly, I explain how these factors affected my research design. Lastly, I reflect on ways my identity influenced my enactment of the researcher role in fieldwork.

I was the oldest child in a Catholic family of eight brothers and sisters. My family lived in a small South Island town and all the children received a Catholic primary school education. Within this setting I received my first lessons about ways of being, such as the importance of prescribed Catholic procedures, and gender roles. For me the messages started to unravel when I struggled to find a place for myself, observed inequities, and began to recognize value in other ways of being.

I left home at seventeen to gain my Bachelor of Commerce and Diploma of Teaching, and became a secondary teacher. My secondary teaching career spanned 22 years and was a great source of stimulation and pleasure to me. I spent 12 years teaching in Porirua, a low socio-economic city north of Wellington, which included large Māori and Pasifika communities. I also lived and taught for two years on Rarotonga, in the Cook Islands (Cl). Many Māori and Pasifika students taught me about social injustices they routinely experienced, most of which I had been privileged to avoid. My prior experience of inequity had been largely on the basis of gender and religion. As the oldest daughter in my family, I fought against unfair treatment of girls, but was deeply shocked as I gained awareness of the struggles of others of Māori and Pasifika ethnicity. The same students also widened my knowledge and experience in other ways; for instance, I learnt from them about Malcolm X, rap, and ways to approach projects collectively. 
While teaching at Porirua College, various professional development opportunities greatly influenced me. For example, AIMHI research (Hawk \& Hill, 2000) confirmed the fundamental nature of good teacher-student relationships for effective work with Māori and Pasifika students. I learnt about peer coaching (Joyce \& Showers, 1980) and its potential for work with professional adult learners. I observed students achieving highly in some areas and failing in others. For seven years, as elected staff representative on the school Board of Trustees, I worked alongside parent and student representatives. Parents and students faced challenges in discussion with high status professionals; however, their perspective was important for the school to develop as a place that met the local community's needs. Due to the principal's vision, both adult and student panels conducted interviews for senior positions, allowing me to witness students' skills and high quality reflective thought.

My awareness and appreciation of deeply embedded social justice issues deepened over the years from personal relationships with individuals from various cultural backgrounds; walking alongside non-Pākehā loved ones has afforded me glimpses of life in their shoes, and shown me responses to their difference.

Currently I am employed as a lecturer at Victoria University of Wellington. I teach within the Graduate Diploma of Teaching and Bachelor of Arts programs. I wanted this position in order to bring my experiences and learning to teacher trainees. Reflecting on my history, I realized my own upbringing had been monocultural, and in order to be effective as a teacher, I needed to learn about myself and be open to other ways of being. Therefore, in taking up my current job, although I am a Pākehā woman, I hoped my experiences might help trainees to unravel some widely held myths, such as stereotyped ideas and deficit theorizing around Māori and Pasifika students.

Thus I was drawn to design a study that might illuminate benefits arising from teachers learning about Māori and Pasifika students as a way to encourage teacher reflection on beliefs, and move beyond deficit theorizing and essentialized notions of ethnic groups. From my own experience, I believed this work could improve teacherstudent relationships and potentially enhance effectiveness of teaching and learning for minoritized students. I was fortunate to meet Professor Christine Sleeter, who introduced me to the concept of FoK, and I immediately appreciated the alignment of the philosophy and practice of FoK with my own concerns.

Bateson's words, introduced to me by González, resonated for me: 
The encounter with persons, one by one, rather than categories and generalities, is still the best way to cross lines of strangeness. (Bateson, cited by González, 2005, p. 29)

In my life, development of close relationships with "othered" individuals has been a key factor in helping me to understand their reality. Therefore I was attracted to a research approach in which teachers interacted with Māori and Pasifika students in intimate settings. González nuances Bateson's (2000) advice, stating "it is only through face-to-face interaction and one-to-one encounters with persons, through mutually respectful dialog, that we can cross constructions of difference" (González, 2005 , p. 44, italics added), emphasizing the role of openness and respect in creating readiness for new learning about individuals "othered" by society. Therefore, this research question and approach tie together my interests in drawing on perspectives of multiple participants, especially voices of minoritized students, and investigating impacts arising when teachers learn about their FoK.

I brought beliefs, values, and FoK to the researcher role, which influenced my interactions. My teaching background affected how I positioned myself (Davies \& Harré, 1990) with school managers, teachers, and students. My interactions with parents were informed by Board of Trustees experiences, and my identity as mother to my Māori daughter. For example, my previous career as a secondary school teacher, including management and timetabling experience, gave me knowledge about the culture and organization of schools.

My prior experiences working within Māori and Pasifika communities also contributed to my FoK. Thus, starting with recruitment, I worked to build relationships with Māori and Pasifika students, knowing this was a necessary foundation for us to work together (Hawk et al., 2002). Key elements of relationships identified by Hawk et al. (2002) I tried to enact were empathy, caring, respect, going the extra mile, and patience.

The research site was in a community with which I had occasional previous involvement. Earlier, I had three types of professional interaction with school community members: networking and collaboration within professional groups (when I was a secondary teacher); observation of student teachers at the school; and availability as adviser to the Specialist Classroom Teacher as well as economics and accounting teachers. The latter two interactions occurred during my current position at Victoria University of Wellington. On a personal level, I had very few connections with local residents, and had spent no time at local events. Therefore, from the perspective of parents and students, I began this project very much as an outsider. 
As an outsider, I knew I would have to earn participants' respect. Narayan (1993) argued that the key issues are power, reciprocal benefits and relationship, rather than insider-outsider status as such, suggesting that truly respectful methodology enhances validity and reliability.

\subsection{The context of the study}

The purpose of this section is to provide thick, rich description of the study context without compromising participants' confidentiality. Figure 1.1 provides an overview of contextual factors described in the thesis.

My research was undertaken within the Kereru High School (KHS) community ${ }^{6}$, Wellington, NZ. Therefore, information is presented about NZ's national education policy and KHS. Other contextual factors were myself - the researcher (see Section 1.3) - and participants. An overview of participants' demographic profiles is provided in Section 3.7. To provide clarity for the reader, descriptions of participants in each case are provided in Chapters 4-8, each of which is devoted to a case.

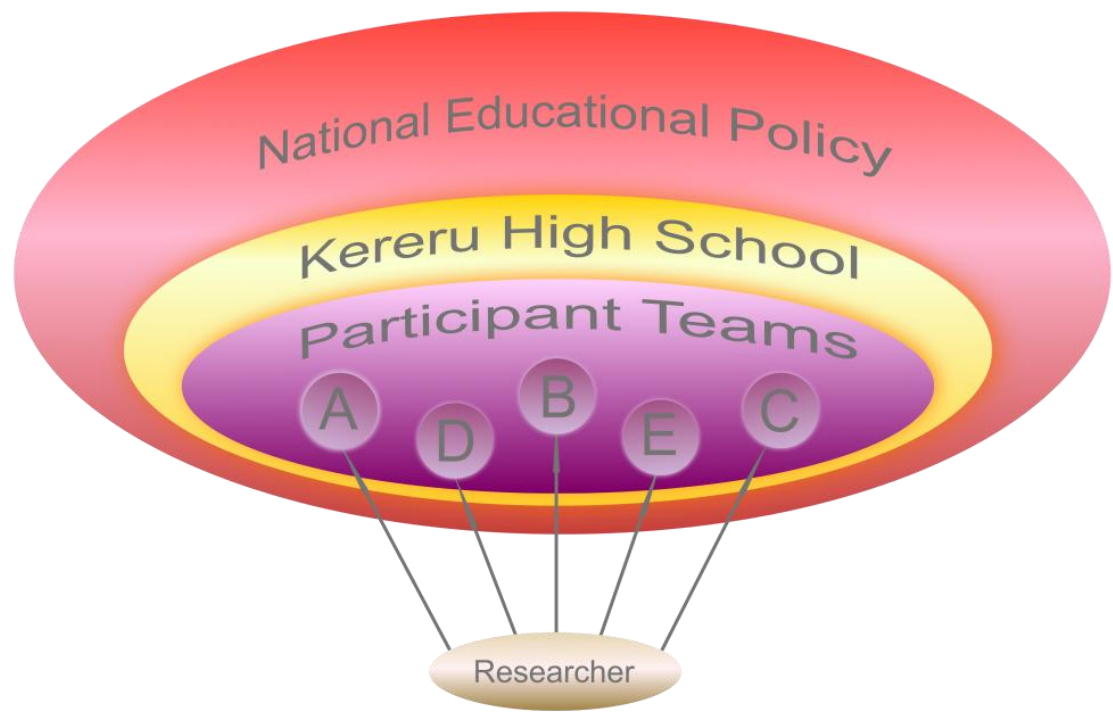

Figure 1.1: Contextual factors

\subsubsection{National educational policies in NZ}

NZ government policy provides strong directives regarding inclusivity for culturally diverse learners, particularly for Māori, the indigenous peoples of NZ, as a key priority. The main points of key documents are described to illustrate current goals, policies, and practices within the NZ schooling sector.

$6 \quad$ All names (persons, places and organizations) are pseudonyms. 


\subsubsection{Ka Hikitia}

Ka Hikitia, the Māori education strategy 2008-2012 (Ministry of Education, 2007b), outlines a vision of schooling in which teachers build on strengths of Māori, and relationships between homes and schools feature collaboration and communication. In practice, Ka Hikitia remains a largely aspirational document.

\subsubsection{Tomorrow's Schools}

The Tomorrow's Schools reforms (Lange, 1988) devolved accountability to schools by establishing school Boards of Trustees (BOTs), which took on a governance role. Elected parents, students, and other community members joined school principals and teachers as BOT members, thus establishing collaboration and shifting decision-making power to the local level. Simultaneously, requirements within National Education Goals (NEGs) and National Administrative Guidelines (NAGs) created school-level accountability for national equity goals.

\subsubsection{NZ Curriculum}

The recently updated NZ Curriculum (Ministry of Education, 2007c) stated "effective pedagogy requires that teachers inquire into the impact of their teaching on their students" (p. 35). The key role of a "focusing inquiry... establishes a baseline and a direction. The teacher utilizes all available information to determine what their students have already learned and what they need to know next" (p. 35), thus informing pedagogical decision-making. This policy thrust was reinforced by NZ Teachers Council (2009) requirements, stipulating teachers must engage in reflective practice, demonstrating competence by applying teacher inquiry.

\subsubsection{Standards for registered teachers}

The Registered Teacher Criteria identify elements of quality teaching in NZ. The overarching statements strongly highlight teachers' responsibility to promote inclusivity, explicitly stating teachers should "promote equitable learning outcomes" (NZTC, 2009, p. 1), specifying that "in an increasingly multi-cultural Aotearoa NZ, teachers need to be aware of and respect the languages, heritages and cultures of all ākonga (learners)" (p. 1). 


\subsubsection{Kereru High School}

\subsubsection{Demographic profile of KHS students}

In 2011, when fieldwork was conducted, KHS was a Decile 4 school $^{7}$ with almost 1,000 students. The main ethnic groups represented in the student population were Māori (42\%), Pākehā (46\%), and Pasifika (10\%).

\subsubsection{Special features of curriculum delivery}

WHĀNAU UNIT

The school's whānau unit ${ }^{8}$ aimed to provide a learning environment specifically tailored to Māori students' cultural identity, which students could opt into instead of mainstream classes.

\section{INTEGRATED STUDIES}

In 2011 KHS operated an integrated studies (IS) program for some mainstream junior classes. IS created a framework whereby common thematic units of study were applied in English, Social Studies, Mathematics, and Science classes. Therefore, learning in four subject areas was linked, to enhance students' experience of congruence across subject disciplines.

\subsubsection{Māori and Pasifika student achievement}

Achievement rates for KHS students in NCEA (at all three levels) were below national and decile means. Therefore, not only were ethnic disparities a nationwide issue, but Māori and Pasifika students at KHS were more at risk of underachievement than their peers at some comparable schools.

\subsubsection{Strategic goals}

The school principal, Anna, stated an overarching strategic school goal was to raise Māori student achievement. She stated within the overarching goal, two objectives were improvement of home-school connections with Māori whānau and learning about how to improve learning for Māori students. Vanessa, the senior manager responsible for teacher professional learning (TPL), noted the school also aimed for improvements in achievement for Pasifika students, and that no student should have to sacrifice cultural identity for academic success.

7 In New Zealand, each school is assigned a decile rating indicative of the extent to which it draws its students from low socio-economic communities. The rating scale sorts approximately $10 \%$ of schools into each of ten deciles: Decile 10 schools draw the least from low socio-economic (SES) communities, whereas decile 1 schools include the largest proportions of students from low-SES communities (Ministry of Education, n.d.).

8 All Māori words are italicized, with the exception of hui, due to its frequency. 
Anna stated, in accordance with the research focus, the school "need(ed) to engage better with the Māori community, to draw in their support, to raise the expectations they have of their children, all these sorts of things" (interview, 14m.51s-15m.03s). Participating in the present study was one of several initiatives Anna hoped would assist progress and generate relevant knowledge:

\begin{abstract}
I see value to us in what you're doing. To me, it will dovetail into some of the things that I want to do with the school around consultation... we've had limited success in the past, and my understanding is that your research is going to help us do that better... it will inform us. (interview, $2 \mathrm{~m} .16 \mathrm{~s}-2 \mathrm{~m} .44 \mathrm{~s}$ )
\end{abstract}

For Anna, the most engaging aspect of the present study was exploration of a team approach, with its focus on both student and parent perspectives. The school had embedded practices that drew on student voice (see Section 1.4.2.5); thus, improving home-school communication and gaining parent voice was their goal.

\title{
1.4.2.5 Teacher professional learning (TPL)
}

BACKGROUND: TE KOTAHITANGA

The Te Kotahitanga project (Bishop et al., 2003) is a large-scale research and TPL project that began in NZ in 2001 . The project has three strands: gathering student narratives and other data related to Māori students' schooling experience; using student narratives and other literature to develop a description of effective practice, known as the Effective Teaching Profile; and using student narratives as a TPL tool.

\section{E TIPU E REA: THE SCHOOL'S LOCAL VERSION OF TE KOTAHITANGA}

Since KHS wanted to be a participating school in the second round of the $\mathrm{Te}$ Kotahitanga project but was ineligible because of its location, a local version was implemented in 2008-9. This program - E Tipu E Rea ${ }^{9}$ - was therefore KHS's "response to Māori underachievement, using the principles of Te Kotahitanga which were really successful up in the North Island, and transferring a lot of the work they did into our school and our context" (Georgia, interview, 3m.42s-4m.03s). Knowledge and tools were gained from annual Te Kotahitanga conferences.

\section{HE KĀKANO}

In 2011, when fieldwork for the present study was conducted, along with a number of schools throughout NZ, KHS was in their second year of participation in $\mathrm{He}$ Kākano, a TPL initiative with a focus on school management systems and structures, to improve their effectiveness for Māori students.

In He Kākano's inaugural year, 2010, the principal, board and senior managers participated, completing an audit of senior management policies and operation. In

9 This name is a pseudonym 
2011 , heads of faculty ${ }^{10}$ participated, auditing faculties to assess the curriculum's cultural responsiveness. To this end, faculties presented student achievement data by ethnicity, and outlined plans to address disparities.

\section{SCHOOL-BASED TEACHER PROFESSIONAL LEARNING (TPL)}

A school-based TPL program maintained a strong focus on inquiry-based TPL. Each year, teachers designed, conducted, and shared findings from an individual inquiry on a topic of their choice. A benefit of this approach, according to Anna, was utilizing teachers' current expertise as a strength. Therefore personalization of individual inquiries was seen as both affirming for teachers and supporting professional learning and enhanced practice.

KHS's approach to individual inquiries incorporated peer coaching and target students. Teachers formed pairs of buddies, and each worked with another buddy pair to enhance accountability. The principal presented inquiry-based TPL as a way to support all teachers to demonstrate registration requirements:

If you're part of our program, and everyone is, and you gather evidence along the way, and we'll talk to them about how we're doing that, then you've got it nailed. Because it's all about reflection, it's all about inquiry, it's all about feedback from students, they just fit together. (Anna, interview, 31m.49s$32 \mathrm{~m} .06 \mathrm{~s})$

Each teacher chose target students, from whom they collected data relating to how their practice was working for them. The principal stated "it's the kids who aren't engaging, they're the ones that we want for your target students" (interview, $27 \mathrm{~m} .01 \mathrm{~s}-27 \mathrm{~m} .08 \mathrm{~s})$. Summing up the underlying philosophy, she stated:

The idea is to get feedback from students. I mean how do you measure your effectiveness unless you ask your audience. (interview, 27m.30s-27m.41s)

Unlike the notion of using students' prior learning, the term or concept of "funds of knowledge" was previously unknown to school managers and teachers involved in data collection.

\subsection{Structure of the thesis}

In this opening chapter, I outlined the rationale for the study and described the research context. In Chapter 2, a narrative literature review provides a critical analysis of scholarly work in the field of FoK, highlighting how research questions addressed in this study will contribute new knowledge. Chapter 3 provides an overview of the research methodology, focusing on features of the design and

\footnotetext{
${ }^{10}$ In NZ schools, a faculty is a grouping of teachers of related subjects. For example, a Science Faculty could include teachers of Biology, Chemistry, and Physics.
} 
procedures that verify trustworthiness of findings. Findings related to each case (Teams A, B, C, D, and E) are presented in separate chapters (Chapters 4-8), in order to allow space for thick, rich description of each team's identity and experience. Cross-case findings and general findings related to the actual experience of fieldwork are discussed in Chapter 9. Finally, Chapter 10 concludes the thesis with a discussion of links between findings and other scholarly work, reflection on limitations of the study, and consideration of implications for further research and teaching practice. It should be noted that although the main body of literature is described in Chapter 2, it became apparent upon analysis that additional literature - relating to student voice, parental involvement, and systems theory was also relevant. Because this was not part of my original thinking, it is introduced in Chapter 10. 


\section{Chapter 2: Literature Review}

In the Māori pupil lies the greatest reservoir of unused talent in the population. (Commission on Education in New Zealand, 1962, p. 414)

Teachers are generally poorly informed about the socio-cultural backgrounds of their students, and... we don't know what difference it would make if they were knowledgeable. (Penetito, 2008, p. 14)

\subsection{Background}

FoK scholarship has its roots in educational anthropology and builds on two lines of research by scholars with social justice concerns (Hogg, 2011). The first line of study dates back to the 1960-70s, when studies of minoritized students' schooling experiences (such as A. R. King, 1967; Rosenfield, 1971; Ward, 1971; Wolcott, 1967) reported findings related to deficit theorizing of these students. Findings of gross inequity within the American schooling system prompted Ryan's (1972) statement that schools were "culturally deprived" (p. 61), and Spindler and Spindler's (1983) assertion of systemic "intense brutality" (p. 75) towards minoritized students by schools. However, despite the rich findings within these qualitative studies, at the time they fell on deaf ears.

The second line of research relevant to FoK work investigated practices of low-SES families, generating new knowledge that exposed the inaccuracy of popular deficit theorists' assumptions. For example, Lewis' (1966) seminal study of American families living in poverty highlighted skills and resources utilized by families to survive. Heath's (1983) important study of literacy practices in low-SES and highSES families not only illuminated literacy practices within low-SES families, but also the significance of congruence (or incongruence) between teachers' and families' questioning style. Heath's groundbreaking work thus reinforced conclusions by Ryan (1972), Spindler and Spindler (1983), and others. Furthermore, Heath demonstrated that when teachers were culturally responsive, by adjusting practice to better match students' home cultures, improved academic achievement resulted.

The term 'funds of knowledge' was introduced by Vélez-lbáñez (1988), building on Wolf's (1966) description of various resources and knowledge that households manipulate to make ends meet in the household economy, which he called 'funds.' For example, two types of funds identified by Wolf were caloric funds (for provision of adequate nutrients) and replacement funds (to maintain assets). Vélez-lbáñez (1988) developed the term 'funds of knowledge' to describe findings from his ethnographic study that investigated practices of economically vulnerable, low-SES 
Mexican families in Mexico and USA. FoK which Vélez-lbáñez (1988) found to be shared by clusters of households related to "a great array of familial, household, neighbourhood, and institutional contexts... (and) included information and formulas" (p. 38) pertinent to household operation and wellbeing. Thus, through exchange within networks, low-SES Mexican families had access to diverse FoK, such as engineering, butchering, job opportunities, and transportation routes. Benefits from FoK included reducing families' reliance on market purchases, and helping them to optimize value from scarce financial resources.

Shortly afterwards, Vélez-lbáñez began to collaborate with other educationalists, including educational psychologists and anthropologists at the University of Arizona, Tucson, Arizona, USA. Thus the group of "Tucson academics" (Hogg, 2011, p. 668) emerged, who led research efforts applying the FoK concept to school settings; Luis Moll, Norma González, James Greenberg, and Carlos Vélez-lbáñez worked with teachers and doctoral students, including Martha Civil, Rosi Andrade, Joel Dworin, Martha Floyd-Tenery, Kathy Whitmore, Cathy Amanti, Douglas Fry, Elizabeth Saavedra, and Javier Tapia. Much FoK research is the work of this group; of the texts analyzed in this literature review, they contributed 11 journal articles, 14 book chapters and the government reports.

As described in Section 1.2.1, recent studies confirm the shameful continuation of problems affecting minoritized students in schools, and the persistence of deficit theorizing. Around the globe, the same students remain disadvantaged in schools, and therefore in later life experiences (e.g., through reduced access to tertiary study and lower earning power). However, given increasing levels of international migration within our shrinking world, a new imperative emerges for this field of work.

\subsection{Literature review methodology}

This narrative review of FoK studies focused on research in school settings that used the term 'funds of knowledge,' and were available in English. Studies in other educational settings (e.g., early childhood, tertiary study, and special education) were excluded. Also excluded were studies related to FoK in fields beyond education, such as medicine.

My search for literature utilized Victoria University of Wellington's library catalogue, as well as a range of electronic databases, including Scopus, Educational Resources Information Centre (ERIC), A+ Education, Proquest, Index New Zealand, and World Cat. I originally sought to identify and draw on peer-reviewed journal articles or books, which provided methodology details as well as reporting on 
research findings related to FoK in school settings, so that rigorous critique could be applied. However, due to a relatively small number of papers which met these criteria, and identification of a significant number of theory or position papers on the topic, I widened my criteria for analysis. Other texts included described research findings (even if methodology information was very brief), as well as those that conceptualized the topic. I argue this is justifiable given that this area of research is still relatively new, resulting in a significant number of position papers seeking to explore the concept's potential. Within these parameters, I located more than 90 texts in the field. These included more than 60 journal articles, 25 book chapters, as well as government reports and conference presentations.

\subsection{Scope of FoK literature}

Analysis of literature revealed current FoK research related to school settings is relatively limited in terms of geographical and educational contexts. I located only 21 texts originating outside USA, including ten from Australia, six from the United Kingdom, two from Canada, and one each from Spain, Finland, and NZ, indicating most FoK theorizing and research has been by Northern American and Australian scholars. The one NZ study related to a school setting that I found was FoxTurnbull's (2012) study of primary school students' application of FoK to support their technology learning.

Texts examined reported on research in a range of settings, including households, households and classrooms, and schools/ classrooms. School settings included bilingual classrooms, elementary schools, middle schools, and high schools. In some texts, the research setting was not identified. This range of research contexts indicates that most knowledge has been developed in the area of elementary and middle school education. I found only thirteen texts related to studies at high school level. Of these, six related to Australian studies, including five reporting on the Redesigning Pedagogies in the North (RPin) project, and the remaining seven related to studies in USA (Bailey, 2011; Camangian, 2010; González \& Moll, 2002; Moll \& Cammarota, 2010; Moll, Soto-Santiago, \& Schwartz, 2013; Lee, 2001; Seiler, 2001). Four literature reviews explore: divergent definitions of FoK (Hogg, 2011); theoretical frameworks (Hogg, 2012); conceptual critique (Oughton, 2010); and pedagogical applications (Rodriguez, 2013). A further three texts were position papers relating to application of the FoK concept to high school learning (Hattam \& Prosser, 2008) and assessment (Klenowski, 2009; Lee, 1998). Therefore, these findings made me wonder about the transferability of a FoK approach at different 
levels of schooling and international settings, and, what elements would support meaningful transfer to NZ high school contexts (Hogg, 2011).

\subsection{Definitions of funds of knowledge}

\subsubsection{FoK: A contested term}

Differences in scholars' definitions of the term 'funds of knowledge' highlight its contested nature. Areas of difference relate to FoK as sources or areas of knowledge, and questions of what knowledge and whose knowledge is included in FoK (Hogg, 2011). Although Tucson academics initially defined FoK in relation to knowledge relevant to household functioning and well-being (e.g., Moll et al., 1990), which helps families to "survive, get ahead, or thrive" (Moll et al., 1990, p. 2), more recent definitions have included knowledge that relates to individual functioning as well (e.g., Moll et al., 1992). Later, González and Moll (2002) defined FoK as "based on a simple premise... that people are competent and have knowledge, and their life experiences have given them that knowledge" (p. 625). The latter definition removes the previous emphasis on FoK as functional, and highlights both the situated nature of FoK, and the role of activity, practices, and routines in its development. All definitions above share a focus on FoK as knowledge and skills developed outside the school setting. For example, Moll et al. (1992) state that FoK are "culturally developed" (p. 134) and arising from "life experiences" (González \& Moll, 2002, p. 625). Aligned with this, Rios-Aguilar (2010) used items from the Latino-Hispanic Household Survey as proxies for FoK. These items provided data related to households' labour history, social interactions, frequent activities, educational experiences, and language use.

In contrast, Kumpulainen and Lipponen (2012) used a definition which varies from this conceptualization; they discuss students' FoK as knowledge developed from class fieldtrips - visits to a science museum and a forest.

Rios-Aguilar, Kiyama, Gravitt, and Moll (2011) observe a link between the centrality of practices in FoK and habitus (Bourdieu, 1977), which I will return to later in this section. González and Moll (2002) align their definition with a processual view of culture, meaning "the everyday lived experiences... the processes of everyday life, in the forms of daily activities" (González, 2005, p. 41). González writes:

We focused on practice - what households actually do and how they think about what they do. In this way, we opened up a panorama of the interculturality of households, that is, how households draw from multiple cultural systems and use these systems as strategic resources. (González et al., 2005, p. 10) 
Thus, the FoK concept sits within poststructuralist perspectives, which reject notions of culture as bound, static, and essentialized. González (2005) argues that this conceptualization:

... allows the possibility of seeing beyond the classroom and glimpsing the circulating discourses and shifting fields of power that shape students' lives. Most importantly, we are able to begin to contextualize the hybridity that emerges from the intersection of diverse funds of knowledge. (p. 44)

Recent work emphasizes the relationship between FoK and households' and individuals' wider social context (González, Wyman, \& O'Connor, 2011; Moll et al., 2013). As such, FoK "are always open to transformation" (Moll et al., 2013, p. 181) to navigate dynamic social, economic, and political circumstances.

In a recent development in conceptualizations of FoK, Zipin, Sellar, and Hattam (2012) suggest FoK includes aspirational imaginings, which they argue could potentially result from a transformative approach to "enable the learners whose lives inhabit abject-liquid spaces to exercise their agency to 'recontextualize' potential new futures from their senses of lived realities" (p. 188). This idea relates to Yosso's (2005) notion of aspirational capital, and arises from Zipin's (2009) main contributions to the field: findings of "abject dimensions" (Zipin et al., 2012, p. 190) in low-SES Australian high school students' lives. In 2009, Zipin coined the term "dark funds of knowledge" (p. 320) to describe knowledge and experience of challenging situations such as violence and discrimination. These findings are in stark contrast to those reported by most FoK scholars, described in Section 1.2.4.

Some scholars (Fitts, 2009; Moje et al., 2004) treat FoK as encompassing both areas of knowledge, in accordance with the above definitions, and sources of knowledge. Moje et al.'s study of Latino youths' FoK identified four main sources of FoK: family, community, popular culture, and peer groups. However, within participatory learning discourse, fusing these concepts does not lead to ambiguity, because learning is seen as action (Sfard, 1998) and:

ongoing learning activities are never considered separately from the context in which they take place. The context, in its turn, is rich and multifarious, and its importance is pronounced by talk about situatedness, contextuality, cultural embeddedness, and social mediation. (p. 6)

This argument is borne out by González et al.'s (2001) finding that mathematical practices applied in households, such as in pattern making, cannot be separated from their context. Therefore, participatory learning theories (e.g., Lave \& Wenger, 1991; Rogoff, 1990) are conceptually aligned with González's (2002) argument about the suitability of a processual approach to culture. Figure 2.1 synthesizes 
these ideas to show the relationship between participatory learning, processual approaches to culture, and the definition of FoK adopted in this study.

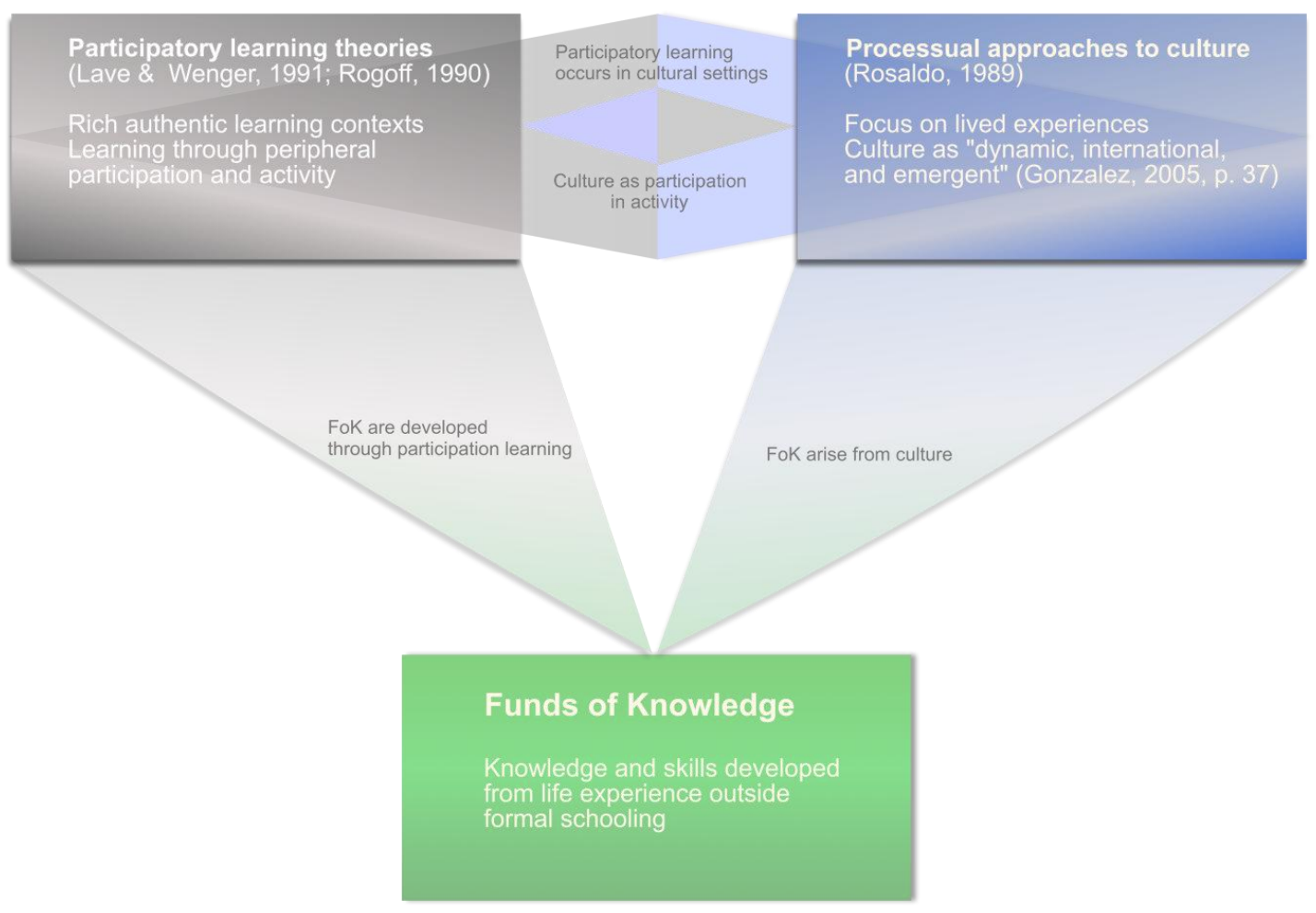

Figure 2.1: Relationship between participatory learning theories, processual approaches to culture, and funds of knowledge

Fox-Turnbull (2012) took a different approach, classifying sources of FoK according to whether they were developed from participatory enculturation (including active participation and peripheral observation) or passive observation (e.g., from watching movies or reading books).

Another area of debate relates to who has FoK. Leading scholars' discourse seems to indicate evolving notions: FoK of households (e.g., Moll \& Greenberg, 1990), students (e.g., Moll, 2005) and individuals in general (González \& Moll, 2002). The question of who has FoK has implications for research methodologies and units of analysis. For instance, Moll (2005) regretted reliance on household ethnographies, which did not fully illuminate students' FoK.

Other views of who has FoK include: networks of households (Vélez-lbáñez, 1988), minority ethnic groups (Andrews \& Yee, 2006), children and young people (Andrade, 1994; Calabrese Barton \& Tan, 2009; Moje et al., 2004; Varelas \& Pappas, 2006), teachers (Hughes \& Pollard, 2006), adults (Bouillion \& Gomez, 2001; Civil \& Bernier, 
2006; Hammond, 2001; Olmedo, 1997), or individuals in general (Basu \& Calabrese Barton, 2007; Fitts, 2009; Rowsell, 2006; Upadhyay, 2005). The definition of FoK applied and conceptualization of who has FoK have implications. For instance, impacts include teachers' views regarding whose expertise can assist scaffolding of new learning. I would argue recognition of parents' FoK potentially allows teachers to demonstrate the value they assign to parents' areas of expertise. However, such an approach could be flawed, if it neglects the relevance of the level and context of planned learning experiences for students. Furthermore, Moje et al. (2004) found that when teachers were unaware of students' FoK, then these could get in the way of new learning, highlighting the potential of a sociocultural approach to learning (Vygotsky, 1978). Therefore, the definition I utilized acknowledges all persons have FoK.

In terms of the issue of whether FoK are located with particular populations, such as minority ethnic groups, Rios-Aguilar et al. (2011) argue that:

Terminology one uses to describe either families' funds of knowledge or individuals' capital is based primarily on social class and the privileges associated with them. 'Funds of knowledge' has become the accepted term for describing capital in lower income and immigrant communities. In addition, it seems likely that funds of knowledge are being traded for traditional forms of capital. (p. 179)

Thus, Rios-Aguilar et al. (2011) highlight issues of "social class, ideology, and power that must situate the understanding of funds of knowledge" (p. 166), in accordance with Bourdieu's (1977) cultural capital theory.

For the purpose of the study, FoK are defined as knowledge and skills developed from life experience outside formal schooling. I decided restricting FoK according to functionality could be cumbersome, and possibly would be seen as arbitrary by participants. I considered it important that the definition was clear and understandable to all team members, since they would collectively determine how teachers would learn about the students' FoK. Also, I was unconvinced by arguments that further restricted FoK, because all areas and sources of knowledge from life experience contribute to students' prior knowledge as they engage in school learning. Furthermore, areas of FoK not seen as purposeful in terms of household or individual functioning may be an individual's passion, giving it high relevance. In constructing this definition, I am locating (but not limiting) the work within the framework of sociocultural learning theory (Vygotsky, 1978).

\subsubsection{Links with related concepts}

My reading of scholarly literature from other fields highlighted some related notions, described in Table 2.1. While acknowledging their conceptual links with FoK, this 
study locates itself specifically within FoK scholarship, due to my interpretation of its distinctive elements, and their alignment with my own FoK and philosophy.

Table 2.1: Related concepts

\begin{tabular}{|c|c|c|c|}
\hline Concept & Reference & Definition or explanation & Comments \\
\hline $\begin{array}{l}\text { Informal } \\
\text { learning }\end{array}$ & $\begin{array}{l}\text { Hager, } \\
2012\end{array}$ & $\begin{array}{l}\text { Informal learning is defined as } \\
\text { oppositional to formal learning. } \\
\text { Features include: contingent, } \\
\text { opportunistic, emergent, tacit, } \\
\text { focused on the learners as } \\
\text { individuals, contextualized, and } \\
\text { holistic }\end{array}$ & $\begin{array}{l}\text { Hager states that informal learning, } \\
\text { historically undervalued, is prominent } \\
\text { in sociocultural learning theories. He } \\
\text { argues that an appreciation of } \\
\text { diverse, complex informal learning is } \\
\text { timely }\end{array}$ \\
\hline $\begin{array}{l}\text { Everyday } \\
\text { knowledge }\end{array}$ & $\begin{array}{l}\text { Flick, 1998, } \\
2006\end{array}$ & $\begin{array}{l}\text { Everyday knowledge is socially } \\
\text { mediated and distributed, as well } \\
\text { as shared, conventionalized and } \\
\text { intersubjective }\end{array}$ & $\begin{array}{l}\text { Flick presents a model to illustrate } \\
\text { the impact of everyday knowledge on } \\
\text { the research process }\end{array}$ \\
\hline $\begin{array}{l}\text { Learning } \\
\text { from our } \\
\text { lives }\end{array}$ & Field, 2012 & $\begin{array}{l}\text { Life is "a permanent process of } \\
\text { learning, which requires constant } \\
\text { reflection on all the institutions } \\
\text { and practices in which we } \\
\text { engage" (Field, 2012, p. 176) }\end{array}$ & $\begin{array}{l}\text { Field's interest here is on } \\
\text { methodologies that investigate how } \\
\text { individuals learn from their lives }\end{array}$ \\
\hline $\begin{array}{l}\text { Community } \\
\text { cultural } \\
\text { wealth }\end{array}$ & $\begin{array}{l}\text { Yosso, } \\
2005\end{array}$ & $\begin{array}{l}\text { Six forms of capital comprise } \\
\text { community cultural wealth: } \\
\text { Aspirational: holding hope despite } \\
\text { barriers; linguistic: languages and } \\
\text { forms of expression; social: } \\
\text { networks and networking; } \\
\text { navigational: operate within social } \\
\text { institutions; familial: from } \\
\text { extended family relationships; } \\
\text { resistance: acquired through } \\
\text { experiences of addressing } \\
\text { inequity }\end{array}$ & $\begin{array}{l}\text { Yosso is a critical race theorist who } \\
\text { developed this model as an } \\
\text { alternative conceptualization of } \\
\text { capital, to disrupt other theories } \\
\text { which privilege White, middle class } \\
\text { knowledge }\end{array}$ \\
\hline $\begin{array}{l}\text { Cultural } \\
\text { capital }\end{array}$ & $\begin{array}{l}\text { Bourdieu, } \\
1977\end{array}$ & $\begin{array}{l}\text { Individuals have habitus: a set of } \\
\text { dispositions that lead to a way of } \\
\text { understanding and interpreting } \\
\text { the world. Class differences lead } \\
\text { to some students having (and } \\
\text { benefiting from) social and } \\
\text { interpersonal dispositions that are } \\
\text { highly valued by institutions in } \\
\text { society }\end{array}$ & $\begin{array}{l}\text { As a critical theorist, Bourdieu is } \\
\text { interested in how habitus reinforces } \\
\text { class structures within society }\end{array}$ \\
\hline $\begin{array}{l}\text { Family } \\
\text { strengths }\end{array}$ & $\begin{array}{l}\text { Harry, } \\
\text { Klingner, \& } \\
\text { Hart, 2005 }\end{array}$ & $\begin{array}{l}\text { Although not defined, reported } \\
\text { findings of family strengths } \\
\text { include parents' commitment to } \\
\text { children's education, parenting } \\
\text { and organizational skills, and } \\
\text { children's courteous, co-operative } \\
\text { and helpful behaviors }\end{array}$ & $\begin{array}{l}\text { Harry et al. are motivated to report } \\
\text { these findings to disrupt persistent } \\
\text { criticism of African American } \\
\text { childrearing practices, and highlight } \\
\text { difficulties these parents face in } \\
\text { utilizing their cultural capital to } \\
\text { support their children at school, } \\
\text { related to their subjection to negative } \\
\text { stereotypes }\end{array}$ \\
\hline
\end{tabular}




\begin{tabular}{|c|c|c|c|}
\hline Concept & Reference & Definition or explanation & Comments \\
\hline $\begin{array}{l}\text { Funds of } \\
\text { identity }\end{array}$ & $\begin{array}{l}\text { Saubich \& } \\
\text { Esteban, } \\
2011\end{array}$ & $\begin{array}{l}\text { Saubich and Esteban suggest } \\
\text { 'funds of identity' to connect FoK } \\
\text { and the identity making process. } \\
\text { They argue "in our view, FoK } \\
\text { involve the material and } \\
\text { resources that people internalize } \\
\text { and use to construct meaning. } \\
\text { Identity is embedded in tangible, } \\
\text { historical cultural factors such as } \\
\text { social institutions, artefacts and } \\
\text { cultural beliefs" (Saubich \& } \\
\text { Esteban, 2011, p. 98) }\end{array}$ & $\begin{array}{l}\text { This work is grounded in } \\
\text { psychological notions }\end{array}$ \\
\hline $\begin{array}{l}\text { Place- } \\
\text { based } \\
\text { education } \\
\text { (PBE) }\end{array}$ & $\begin{array}{l}\text { Penetito } \\
(2008)\end{array}$ & $\begin{array}{l}\text { Penetito (2008) states "the } \\
\text { purposes underlying PBE are } \\
\text { often intended to satisfy } \\
\text { indigenous peoples' aspirations in } \\
\text { education as a priority... a } \\
\text { second important category of } \\
\text { literature on PBE revolves around } \\
\text { issues of ecological literacy, } \\
\text { community studies, the specific } \\
\text { relationship between place and } \\
\text { space, the relationship between } \\
\text { place and identity, and the politics } \\
\text { involving biotechnology and } \\
\text { biodiversity" (p. } 6 \text { ) }\end{array}$ & $\begin{array}{l}\text { Penetito argues that PBE addresses } \\
\text { the concern expressed by Watson } \\
\text { (1967): "Not in New Zealand or } \\
\text { anywhere else does there appear to } \\
\text { have been a systematic, carefully- } \\
\text { disciplined effort to test whether a } \\
\text { teacher's knowledge of the local } \\
\text { scene or his participation in it, has } \\
\text { any significant effect upon his interest } \\
\text { or capacity to integrate this } \\
\text { knowledge into the scholastic work of } \\
\text { his classroom, or into the emotional } \\
\text { toning of his relationships with pupils" } \\
\text { (p. } 40 \text { ) }\end{array}$ \\
\hline
\end{tabular}

FoK scholarship focuses on social justice aims, specifically to develop knowledge of how education practitioners can "exploit fully cultural resources... of communities surrounding schools - their expertise, knowledge and artefacts - and utilize these in a systematic manner" (Kumpulainen \& Lipponen, 2012, p. 112). A key issue of interest to many FoK scholars is the inequity arising in schools due to lack of recognition and respect for FoK of minoritized students. For example, although minoritized students have relevant prior knowledge, standard assessment techniques do not take account of Latino students' FoK; consequently, culturally biased assessment practices result in over-representation of Latino students in remedial programs (Rios-Aguilar, 2010).

Thus, contributions to knowledge related to FoK of minoritized students have the potential to support the breakdown of systemic issues that contribute to academic underachievement within these groups. (See Section 2.7 for discussion of valued outcomes from identification of minoritized students' FoK.)

One underlying aim of the FoK approach to teaching and learning is to make schooling more relevant for minoritized students (Moll \& González, 2004). Although place-based education also has this aim, it puts the focus on place rather than on skills and strengths from people's activities. 


\subsection{Theoretical frameworks for funds of knowledge scholarship}

As noted previously, the definition of FoK applied in this study locates it within sociocultural learning theories. Together with critical theory, this was the most popular theoretical framework for FoK scholars, with many situating the work within both frameworks (Hogg, 2012), as shown in Figure 2.2.

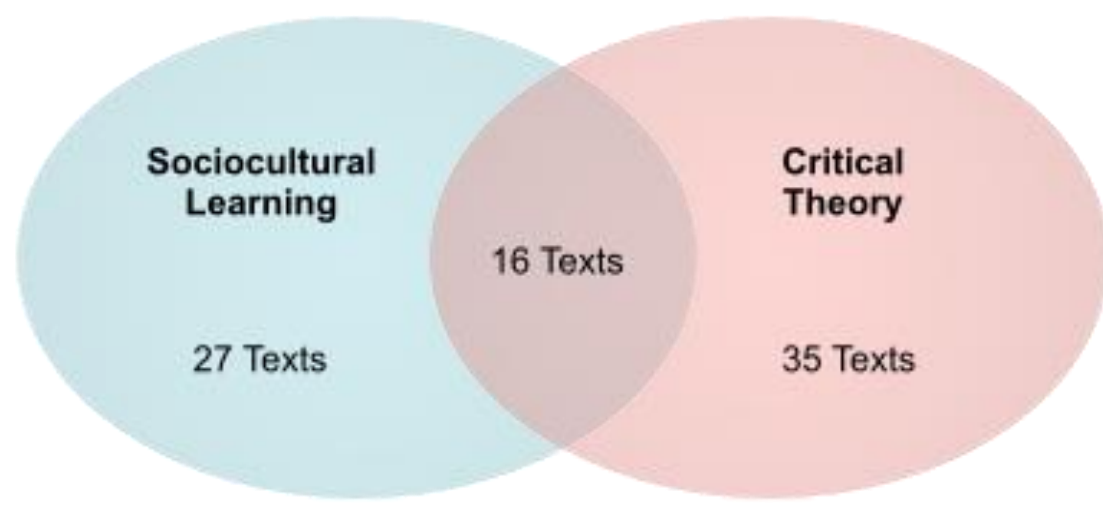

Figure 2.2: Conceptual bases identified in funds of knowledge texts

Divergent thinking regarding the theoretical basis of FoK work evident is unsurprising, given differences in FoK definitions. Other conceptual frameworks cited are hybridity theory (e.g., Calabrese Barton \& Tan, 2009; Fitts, 2009; Moje et al., 2004), systems theory (Patterson \& Baldwin, 2001), and difference theory of caring (Antrop-González \& De Jesus, 2006).

My own understanding of how application of FoK supports learning aligns with Vygotsky's (1978) sociocultural learning theory, which I argue is appropriate for the exploratory nature of the present study. Vygotsky perceives "learning and development as dynamic processes, social, cultural and historical by nature, and in a dialectical relationship with each other" (Wink \& Putney, 2002, p. 62). Moll and Whitmore (1993) assert understanding and applying the zone of proximal development must take this into account, recognizing the importance of social interaction and semiotic tools, together with "a focus on meaning" (p. 39), compatible with Vygotsky's (1978) views of humans acting to transform their lives, assisted by cultural tools. Within this, students' life goals and values - which provide the purpose of learning - are of paramount importance, influencing perspectives of the relevance of specific learning experiences. I agree with Erickson's (2007) analysis that elements of students' lifeworld experience form highly personalized sets of communities of practice (CoPs), each with its own activity focus and discourse, including associated inter-personal and intra-personal communication: ways of talking, thinking, interacting, reading, and writing (Gee, 1996, 1999). Within CoPs, learning occurs with support of more expert individuals, who may be peers, 
parents, community members, or teachers. Fitts (2009) points out it is important for minoritized students to be positioned as experts in school, to redress historical inequity arising from neglect of their perspectives and offer opportunities for dominant culture students to develop new understandings. Therefore, within sociocultural learning theory, teachers have a pivotal role as designers and managers of learning experiences and classroom interaction.

Hybridity theory (Bhabha, 1994) has close links with sociocultural learning theory, because of its focus on dialogic interaction, and notions of third space. All FoK scholars who locate the work within hybridity theory (Calabrese Barton \& Tan, 2009; Fitts, 2009; Moje et al., 2004) also identify sociocultural learning theory as a conceptual framework. Hybridity theorists identify first space as knowledge and discourses from home, community, and peer groups. Second space includes knowledge and discourses from formalized institutions such as schools and churches. Three views regarding the role of third space (also called hybrid space) in promoting academic achievement are all relevant to FoK work (Calabrese Barton \& Tan, 2009; Fitts, 2009; Moje et al., 2004). They are:

- To scaffold academic learning through making connections to FoK

- To support development of expertise in new CoP discourses

- To allow competing discourses and knowledges to be addressed.

The latter function is most relevant for minoritized students, due to incongruency between their CoPs and dominant discourses (Moje et al., 2004). It is also the most challenging for teachers to implement (Fitts, 2009).

\subsection{Approaches to identify students' funds of knowledge}

Diverse methods employed in studies to identify students' FoK and research settings are summarized in Table 2.2. 
Table 2.2: Methodologies employed or described to identify students' funds of knowledge

\section{Methodologies}

Research examples

School setting

Researchers conduct ethnographic interviews in households, discuss findings and Moll et al., 1990

collaborate with teachers in study groups to consider implications for practice, classroom Moll \& Greenberg, 1990 observations

"The funds of knowledge methodology" (González, 1995, p. 238): teachers conduct Moll, 1992

ethnographic interviews in households, discuss findings collaboratively and consider Moll et al., 1992

implications for practice in study groups, classroom observations

González et al., 1995

Patterson, Baldwin, Gonzales, Guadarrama, \& Keith, 1999

González et al., 2005

Patterson \& Baldwin, 2001

Floyd Tenery, 2005

Amanti, 2005

Hensley, 2005

Sandoval-Taylor, 2005

Browning-Aiken, 2005

Messing, 2005

Brenden, 2005

Mercado, 2005

Ethnographic interviews in households

Vélez-lbáñez \& Greenberg, 1992

lementary \& middle

Elementary \& middle

\section{Home visits, including use of other instruments}

(such as surveys, self-portrait, self-definition)

Andrews \& Yee, 2006

Ayers, Fonseca, Andrade, \& Civil, 2001

Saubich \& Esteban, 2011

Cremin, Mottram, Collins, Powell, \& Drury, 2012

Macias, 2013

Middle

Middle

Kindergarten \& elementary

Elementary

Kindergarten, elementary \& middle

Elementary

Unstated

Unstated

Elementary

Elementary

Middle

Elementary \& middle

Unstated

Elementary

McIntyre, Kyle, Moore, Sweazy, \& Greer, 2001

Family visits

Edwards, Pleasants, \& Franklin, 1999

(Households)

Elementary

Collecting parents' stories

Comber \& Kamler, 2007

Middle

Unstated

Elementary/kindergarten

Unstated

Literacy audit and case study of a student

Elementary

Meetings to discuss identified topics

Riojas-Cortez \& Flores, 2009

Elementary

Observation, surveys, interviews and artefacts (such as photos and documents)

Moje et al., 2004

Elementary

Conducting a community scan, with some involvement of students as researchers

Smythe \& Toohey, 2009

Middle

Neighbourhood walk with student

Sugarman, 2010

Elementary

Conducting a participatory sewing circle

González et al., 2001

Elementary

(Community) 


\begin{tabular}{|c|c|c|}
\hline Methodologies & Research examples & School setting \\
\hline Assignment of autobiographical tasks or community based inquiry & $\begin{array}{l}\text { González \& Moll, } 2002 \\
\text { Rogers, Light, \& Curtis, } 2004 \\
\text { Camangian, } 2010\end{array}$ & $\begin{array}{l}\text { High } \\
\text { Elementary } \\
\text { High }\end{array}$ \\
\hline Assignment of oral history tasks & $\begin{array}{l}\text { Olmedo, } 1997 \\
\text { Flores Carmona \& Delgado Bernal, } 2012\end{array}$ & $\begin{array}{l}\text { Unstated } \\
\text { Elementary }\end{array}$ \\
\hline Designing learning which calls on students to apply their cultural knowledge & Maher, Epaloose, \& Tharp, 2001 & Middle \\
\hline $\begin{array}{l}\text { Designing learning which calls on students to write about personal experiences of family } \\
\text { members }\end{array}$ & $\begin{array}{l}\text { Dworin, } 2006 \\
\text { González \& Moll, } 2002\end{array}$ & $\begin{array}{l}\text { Elementary } \\
\text { High }\end{array}$ \\
\hline Designing learning which calls on students to reflect on and discuss personal experiences & $\begin{array}{l}\text { Calabrese Barton \& Tan, } 2009 \\
\text { González \& Moll, } 2002\end{array}$ & $\begin{array}{l}\text { Middle } \\
\text { High }\end{array}$ \\
\hline Designing learning which allows students to choose learning context and process & Basu \& Calabrese Barton, 2007 & Middle \\
\hline $\begin{array}{l}\text { Read-alouds of informational texts and encouragement of student comments and } \\
\text { observations }\end{array}$ & Varelas \& Pappas, 2006 & Elementary \\
\hline $\begin{array}{l}\text { Creation of a safe learning environment where a teacher also shares personal funds of } \\
\text { knowledge }\end{array}$ & Upadhyay, 2005 & Elementary \\
\hline $\begin{array}{l}\text { Asking students, with the help of family members, to collect and present significant } \\
\text { artefacts }\end{array}$ & $\begin{array}{l}\text { Hughes \& Greenhough, } 2006 \\
\text { Hughes \& Pollard, } 2006\end{array}$ & $\begin{array}{l}\text { Elementary } \\
\text { Elementary }\end{array}$ \\
\hline Asking students to collect and explain significant artefacts & Zipin, 2009 & High \\
\hline Students use of sketchbooks and writer's notebooks & Thomson \& Hall, 2008 & Middle \\
\hline Encouraging students to talk about their lives; Students create clay animation videos & Henderson \& Zipin, 2010 & High \\
\hline Class brainstorm and survey & Fraser-Abder, Doria, Yang, \& De Jesus, 2010 & Middle \\
\hline Science lunch group & Seiler, 2001 & High \\
\hline Class discussion of local issues & $\begin{array}{l}\text { Zipin, } 2009 \\
\text { Zipin et al., } 2012\end{array}$ & $\begin{array}{l}\text { High } \\
\text { High }\end{array}$ \\
\hline Employment of teacher aides from same culture as minority students & Martin-Jones \& Saxena, 2003 & Elementary \\
\hline Calling for offers of parental involvement & Kahn \& Civil, 2001 & Elementary \\
\hline Extrapolating FoK information from Latino/Hispanic Household Survey & Rios-Aguilar, 2010 & \\
\hline
\end{tabular}


One method which has been very influential and successful in the field incorporates home visits. This method was designed and refined by Moll, González, and colleagues (see Moll et al., 1990; Moll et al., 1992), and, was termed "the funds of knowledge methodology" (González, 1995, p. 238). In this model, teachers (with researchers' support) collect data during home visits, using an ethnographic approach. Key features fundamental to the approach's success are identified as:

- Before home visits are conducted:

○ training in ethnography, including readings and practice

- Over the course of home visits:

- questionnaires which provide guidelines for interviews

- clear expectations for fieldnotes

- study group sessions for teacher participants and researchers (González et al., 2005)

In study group sessions, Moll reports:

We discuss the background readings, introduce observations and note-taking, revise interview procedures, review findings from each visit, and discuss classroom practices and implications. (González et al., 2005, p. 17)

Study groups establish an environment where sociocultural learning practices can be activated within the group of teachers and researchers, thus fulfilling a 'mediating' function (Vygotsky, 1978), supporting teachers to gain an understanding of FoK from data collected and consider implications for practice. Moll notes:

It is through the process of writing field notes and discussing them that one gives theoretical form and substance to the connections forged empirically between the households and the teachers. (González et al., 2005, p. 19)

Thus, a major contribution of this work to the field was the development and full description of a research model, including detailed discussion of the theoretical basis and methodology, which allows replication and supports achievement of valued outcomes. This work highlights challenges teachers face when they take on a research role, and identifies strategies that have proven to be successful for teacher participants (e.g., Amanti, 2005; Floyd Tenery, 2005; Hensley, 2005; Messing, 2005). However, Baquedano-López, Alexander, and Hernández (2013) see researchers' mediation as problematic, interpreting it as patronizing to parents.

An alternative model for providing support to teachers undertaking FoK work was offered by Cremin et al. (2012). In this work, teachers were provided six days of professional development to develop researcher knowledge and skills, and linked 
researchers supported teachers to interpret information about students and families. One point of difference was that teachers were not accompanied by researchers when conducting home visits. This study design feature related to the researchers' goal to implement an approach that might be sustainable for teachers in the long term, due to its lack of reliance on external support (Cremin et al., 2012).

Apart from the possible unfamiliarity of undertaking research outside their own classrooms, arguably most teachers are unused to observing students or others as investigators of FoK, which adds a further layer of challenge (Cremin et al., 2012). Factors that could create challenges for teachers as they look at students with new intent include their own social interaction style and beliefs about students and families. Therefore, I decided it was important for me to include a study group forum for teacher participants. Although resource constraints affected their form in this study, my planning was informed by key features of teachers' study groups (See Chapter 3).

Many published works relating to application of the funds of knowledge methodology" (González, 1995, p. 238) describe studies in Arizona, USA where leading scholars Moll and González are based, and further afield in USA (e.g., McIntyre et al., 2001; Mercado, 2005; Patterson et al., 1999). Studies applying this method have all related to elementary or middle school settings. Mclntyre et al. (2001) adapted the model slightly, using the term family visits to place the focus on the people and off the home. Like González et al. (2005), they found some families did not want home visits; some preferred to meet on their porch, or at a completely different location. In Spain, Saubich and Esteban (2011) employed a range of psychological tools in home visits, including art-based tools, rather than ethnographic interviewing.

Riojas-Cortez and Flores (2009) used an approach in which parents met regularly to discuss their knowledge about a set of pre-determined topics, such as play and literacy, scientific cultural literacy, and literacy within the home. As for ethnographic home visits conducted in González et al.'s (2005) FoK methodology, discussion related to pre-set topics, although the topics themselves differed. Unique features of this approach included: meeting with multiple parent participants to collectively discuss and affirm their FoK, the research setting, and the use of activities after discussions that involved parents and children together (which proved to be a motivator for participation). Activities included playing traditional Mexican games and parents and children writing cartitas de carino (affection notes) to each other. 
A number of studies identified students' FoK by assignment of tasks requiring students to draw on their own, family members' or community members' FoK. These included oral history tasks (Olmedo, 1997), movie production (Bailey, 2011), writing tasks (Camangian, 2010; Dworin, 2006), research for a video production (PirbhaiIllich, 2010), and reporting on home nutrition practices (Calabrese Barton \& Tan, 2009). Arts-based techniques included use of sketchbooks and notebooks (Thomson \& Hall, 2008) and production of clay-animation videos (Henderson \& Zipin, 2010). Diverse examples demonstrate how learning activities in various subject disciplines can usefully draw on students' FoK.

González and Moll (2002) argue that study of community FoK is highly suitable for high school students. Moll and Cammarota (2010) describe participatory action research projects undertaken by high school students that added to students' FoK by shifting their thinking:

... past self-condemnation for unjust social realities to the realization that
subordination or secondary status results from institutional or systematic
constraints. Engaged praxis requires students to use this realization
specifically for change by learning how their situation or reality is not fixed but
indeed malleable with direct challenges to oppressive barriers. (Moll \&
Cammarota, 2010, p. 298)

Study of community FoK has also been successfully used at elementary level (Flores Carmona \& Delgado Bernal, 2012; Rogers et al., 2004). Smythe and Toohey (2009) engaged a class of elementary students as researchers, and collaboratively identified and mapped community resources, thus focusing on place-based assets, rather than FoK held by people.

However, Zipin et al. (2012) found that in their research setting, north of Adelaide, Australia, their assumption of community, following Gutiérrez and Rogoff's (2003) definition - "a co-ordinated group of people with some traditions and understandings in common" (cited in Zipin et al., 2012, p. 183) - was not applicable. The area included migrants and refugees from diverse countries, within which there were some small cohesive communities, but the local population was "in flux, unsettled, often internally diverse, sometimes fractiously so" (Zipin et al., 2012, p. 183), exemplifying the dynamic nature of "liquid modernity" (Bauman, cited in Zipin et al., 2012 , p. 184). When students made photomontages of their community, "highly disparate testimonies" (Zipin et al., 2012, p. 184) were revealed, which did not allow for neat synthesis. Therefore, the researchers changed the unit of analysis from community to lifeworlds: "spaces where 'kids hang out together' " (Zipin et al., 2012, p. 185). However, the change created "risks of diminishing comprehension of the macro-meso-micro scalar complexities and depths" (Zipin et al., 2012, p. 185). 
Therefore, discovery - through students' FoK - of multiple perspectives within a complex and diverse community challenged researchers' and teachers' conceptualization of community FoK.

Some studies utilized approaches focused on establishing an environment conducive to drawing out students' FoK. Methods included provision of bi-lingual teacher aides from the same culture (Martin-Jones \& Saxena, 2003), calling for parental involvement (Kahn \& Civil, 2001), encouraging student comments (Henderson \& Zipin, 2010; Varelas \& Pappas, 2006), and teacher modeling of personal sharing by talking about their FoK (Upadhyay, 2005). Because they all focus on enhancing safety in the classroom environment as a way to identify students' FoK, I regard them as indirect approaches. I also have a concern about the ability of these strategies alone to effectively draw out all students' voices, because of diverse factors which affect students' likelihood to volunteer information and get teacher attention. This was borne out by Henderson and Zipin's (2010) finding that students were "initially dubious and tentative about revealing substantive dimensions of their lives" (p. 189). Because different students experience different treatment and outcomes at school (e.g., Bishop \& Berryman, 2006; Sleeter, 2011), I am sceptical that this approach would be sufficient to draw out students' FoK, without teacher skill, reflexivity and commitment to social justice aims. Woodrow (2013) found that although teachers asked about students' FoK, they quickly reclaimed center stage and did not allow space for students to discuss their FoK and relate it to the learning. Therefore, it seemed that greater intentionality was necessary for FoK to be integrated into classroom learning experiences. However, Seiler's (2001) study had some distinctive features: the researcher met with male African American high school students within a Science lunch group setting, to talk about Science and doing Science activities. Thus, this research setting included fewer members, who had all volunteered, and had a defined purpose.

Sharing significant artefacts within class settings was used to learn about elementary (e.g., Hughes \& Greenhough, 2006) and high school students' FoK (Zipin, 2009). This approach gives students greater autonomy than other methods. Potentially, its strength could arise from this feature - students can choose meaningful artefacts to share. However, constraints such as security worries or fear of putdowns may prevent selection of highly meaningful artefacts. Therefore, this approach would require teacher skill and planning to minimize such risks. Also, artefacts privilege material possessions, which arguably may be seen to reinforce the high value that society places on socio-economic status, and may disadvantage the very students whose FoK with whom the teacher is least familiar. 
I sorted research methodologies to identify students' FoK into five categories:

1. Ethnographic interviews in households

2. Class activities related to students' and community members' FoK

3. Class activities related to local community

4. Students bring artefacts to school

5. Other

This analysis highlights the influence of diverse research traditions. For instance, Category 1 studies drew on ethnographic traditions from anthropology. Some Category 2 studies utilized oral history activities with students, thus drawing on history methodology. Within Category 5, Saubich and Esteban (2011) utilized various psychological tools.

Diverse approaches used raises questions about multiple perspectives relating to, and relative transferability and manageability of, different possible approaches in different contexts. Although home visits were highly valued by families in Tucson, Arizona, USA (Floyd Tenery, 2005), two academic Māori parents in Wellington, New Zealand stated they would not welcome this type of visit by a teacher (personal communication, September 10, 2008), highlighting the existence of multiple perspectives regarding approaches for learning about students' FoK. These questions informed my decision to create a research design that drew out perspectives of different stakeholders, whom I identified as teachers, students, and parents. Thus, my aim was to avoid making assumptions about participants' views, by incorporating a process which illuminated and respected their perspectives. Therefore, my role included informing participants about all approaches to learning about students' FoK that I knew of. After this training, each team selected approach(es) they wished to apply. (See Section 3.8 for research procedures, and Section 9.2.2 for selected approaches.)

\subsection{Valued outcomes arising when teachers learn about students' FoK}

\subsubsection{Illumination of rich funds of knowledge}

FoK research has tended to investigate and apply the concept in studies of minoritized groups. As evidenced by the following examples, many studies involve Latino participants, and attest to rich FoK held by Latino students (Macias, 2013; Moje et al., 2004; Moll, 1992), households (Browning-Aiken, 2005; Cremin et al., 2012; Floyd Tenery, 2005; González et al., 1995; Gutiérrez, 2002; Mercado, 2005; Moll et al., 1990; Moll et al., 1992; Moll, Vélez-lbáñez, \& Greenberg, 1989; Olmedo, 1997; Patterson \& Baldwin, 2001; Sugarman, 2010; Vélez-lbáñez \& Greenberg, 1992), parents (Riojas-Cortez \& Flores, 2009), para-educators (Monzo \& Rueda, 
2003), and communities (Bouillion \& Gomez, 2001; González et al., 2001; Moll \& Greenberg, 1990; Vélez-lbáñez, 1988).

One key finding from investigations of FoK of Latino families relates to the role of reciprocal social networks which contributed to all households' FoK, stressing that FoK are not always held within individuals or families, but may be available through exchange (e.g., Vélez-lbáñez \& Greenberg, 1992).

Other work highlights FoK held by diverse groups, including: Aboriginal Australians (Pirbhai-Illich, 2010), African Americans (Lee, 2001; Rogers et al., 2004; Seiler, 2001), Appalachians (Mclntyre et al., 2001), Haitians (Conant et al., 2001), Moroccans (Saubich \& Esteban, 2011), Native Americans (Maher et al., 2001), Punjabi-Sikhs (Marshall \& Toohey, 2010; Smythe \& Toohey, 2009), Vietnamese (Patterson \& Baldwin, 2001), and low-SES students in Britain (Andrews \& Yee, 2006) and Australia (Henderson \& Zipin, 2010; Ovsienko \& Zipin, 2007; Zipin, 2009). A further area of interest relates to FoK of students whose lives are transnational (Moll et al., 2013), building on social justice concerns related to Latino students whose lives are complicated by sociopolitical elements such as illegal immigration.

\subsubsection{Changes in teacher perceptions}

For teachers, participating in home visits and engaging with information about the reality of students' lives and FoK has been transformative, even when the research has been conducted "in his or her own back yard...(by teachers) trying to consider a familiar context with the new eyes of a trained observer" (Messing, 2005, p. 184). Comber and Kamler (2007) refer to this as teachers turning around to students, using a metaphor which highlights change in teacher attitude, contrasting with the common phrase to turn students around, which locates change with the student.

Inaccurate assumptions were disrupted, leading to more appreciative views of families (Comber \& Kamler, 2007; Cremin et al., 2012; Floyd Tenery, 2005; Hensley, 2005; Patterson \& Baldwin, 2001) and communities (Sugarman, 2010). In many studies, teacher participants retrospectively recognized their unconsciously held deficit perspectives. Comber and Kamler's (2007) methodology included engaging teacher participants in cross-generational interviews early in the project, which surfaced beliefs and provided the basis for further reflection after learning about FoK.

Examples of teachers' statements highlight the impact of involvement in FoK research on their beliefs:

I didn't realize it at the time, but I used to believe that my students had limited opportunities in life. I thought that poverty was the root of many of their 
problems, and that this was something too big for me to change as a teacher. (González et al., 1995, p. 451)

I think that's one thing that we as teachers - I think we're guilty of judging the kids and maybe even looking down on them - not seeing them as equals but as inferior to us because of the kinds of - the class of society that they may come from. I never intentionally judge the kids, not doing it intentionally and this helped me so much to realize that kids are kids - no matter what socioeconomic level they're from and that we need to encourage them in every way. And these visits helped to do that. It gave credence to what they do at home. I learned to value everything that they did at home. (Messing, 2005, p. 189)

This teacher appeared to also gain an enhanced appreciation of herself as a cultural being, as shown by her comment that she became more tolerant of various behaviors in the classroom, knowing "I need to be more understanding, because my background is very different from their background" (Messing, 2005, p. 190). This understanding is foundational for teachers' development as effective multicultural practitioners (Banks, 2001; King, 2004; Sleeter, 2008; Valli, 1996). Teachers' FoK research changed their perceptions of families and students and generated new notions of how to approach teaching (Messing, 2005; Patterson \& Baldwin, 2001).

Teachers also gained deeper understandings of historical (Browning-Aiken, 2005) and sociopolitical factors affecting families' lives (Floyd Tenery, 2005). Shifts in thinking created discomfort for teachers, as they were forced to confront inaccurate notions. One teacher mused, "why did it take me so long to get to where I am now?" (Patterson \& Baldwin, 2001, p. 131).

In her study of seven teachers' evaluations of their participation in "the funds of knowledge methodology" (González, 1995, p. 238), Messing (2005) concluded that teachers' new perspectives led to "a questioning of established roles and understandings. It is this questioning that has the potential for leading to educational change" (p. 184). However, deeply embedded beliefs proved hard to change and some teachers found it challenging to shift their focus to FoK (Comber \& Kamler, 2007; Riojas-Cortez \& Flores, 2009). The following extract illustrates Nola's learning about Ewan's FoK:

He goes fishing and crabbing with his dad most weekends, and his uncle, so he's extremely involved in it and very knowledgeable as well. He can tell you every part of the fishing rod and the sort of fish and the beaches and the lakes, and... his oral language is very, very good. (Comber \& Kamler, 2007, p. 305)

Nola did not maintain a focus on Ewan's FoK in her final written analysis of the case study, showing a continuing reliance on deficit terms. For example:

He's not risk taker (sic).

He won't even attempt to do anything. (Comber \& Kamler, 2007, p. 305) 
Therefore, for some teachers, discordant beliefs remained intact despite learning about students' FoK.

\subsubsection{Closer relationships}

When teachers learnt about students' FoK through home visits, student-teacher relationships improved. Teachers reported placing a greater value on students and teachers understanding each other as people, and breaking through traditional teacher-student relationships to achieve this (Amanti, 2005), as well as feelings of connection (Riojas-Cortez \& Flores, 2009), excitement and pride (Comber \& Kamler, 2007), and stronger commitment to students (Hensley, 2005; Mclntyre et al., 2001).

Impacts also extended to parent-teacher relationships. Fundamental to this shift was the focus on teacher as learner, not "imparter of the knowledge type person" (Messing, 2005, p. 185-186). For some teachers conducting home visits, parents' appreciation of their interest was immediately apparent. For example, Cremin et al. (2012) reported that in one family, both parents changed their work shifts to be at home for the teacher's visit. Floyd Tenery (2005) stated family members farewelled her with a care package of home-cooked food. Hensley (2005) reported the "climate of the teacher-home relationship" (p. 146) was positively affected by teachers' appreciation of FoK, because parents began to feel valued. Exchange and reciprocity were key elements in building rapport with parents (Cremin et al., 2012; Messing, 2005) and students (Flores Carmona \& Delgado Bernal, 2012), and allowed both trust and friendship to blossom. Amanti (2005) reported:

Parents whose homes we visit drop by in our classrooms to make social visits or to extend invitations to our students' birthday parties or First Communion celebrations. New babies are brought by for us to meet. (p. 140)

Furthermore, when teachers learn about students' FoK, students felt more valued, and this impacted positively on their learning (Comber \& Kamler, 2007; Sugarman, 2010) and made them more self-assured in class (Cremin et al., 2012). For instance:

Not only has the length of Willem's writing increased, but traces of Marc's home visit are visible in that Willem now sees going to the football and events with his extended family as legitimate writing material - in part, because his teacher valued his lifeworld outside school. (Comber \& Kamler, 2007, p. 300)

Camangian (2010) found that high school students valued their teacher's interest and care in their problems, as well as the opportunity to reflect deeply on lessons from their life experiences. Hensley (2005) reported she approached students differently, enacting an ongoing search to deepen her knowledge. For instance: 
A child recently came to school wearing a T-shirt with a handpainted cactus. Prior to being involved in this project, my response to this shirt would have been to say, "Isn't that a cute shirt!" Instead, I wanted to know where he got it and who made it. (p. 147)

This finding highlights a key difference between this conceptual framework and other notions of knowing students, which do not specifically value students' and families' strengths and skills. However, the different approaches taken by Camangian (2010) and Hensley (2005) exemplify debate within the field regarding the scope of relevant FoK, especially "dark" FoK (Zipin, 2009, p. 320).

Flores Carmona and Delgado Bernal (2012) argue that relationships between teachers, students and parents in FoK work are based on solidarity, in accordance with Nieto's definition:

Solidarity with and empathy for students are not simply sentimental emotions. For teachers who think deeply about their work, solidarity and empathy mean having genuine respect for their students' identities - including their language and culture - as well as high expectations and great admiration for them... it means trusting them. (Nieto, cited in Flores Carmona \& Delgado Bernal, 2012, p. 119)

\subsubsection{Applying FoK to classroom pedagogy}

Teacher participants' new knowledge of rich and diverse FoK within students' families led to "redefinition of local households as containing important social and intellectual resources for teaching" (González et al., 1995). Training and collaborative reflection in study groups were key elements that supported teachers' success in learning about FoK and applying it to classroom practice (Brenden, 2005; González et al., 1995; González et al., 2005; Messing, 2005).

\section{STRATEGIES FOR IMPROVING PEDAGOGICAL PRACTICE}

Learning about students' FoK helped teachers improve their pedagogical practice (Amanti, 2005; Browning-Aiken, 2005; Comber \& Kamler, 2007; Fraser-Abder et al., 2010; Gutiérrez, 2002; Hensley, 2005; Messing, 2005; Patterson \& Baldwin, 2001; Sandoval-Taylor, 2005; Sugarman, 2010). These may be classified according to Rodriguez's (2013) typology of pedagogical approaches utilizing FoK, summarized in Table 2.3.

Table 2.3: Classifications of pedagogical applications of FoK (Rodriguez, 2013)

\begin{tabular}{lll}
\hline \multicolumn{2}{c}{ Three key themes: } & \multicolumn{1}{c}{ Explanation: } \\
\hline 1 & $\begin{array}{l}\text { Co-construction of knowledge to } \\
\text { deepen or extend students' academic } \\
\text { understanding through FoK. }\end{array}$ & $\begin{array}{l}\text { "Pedagogy as perspective and relevance across social/ } \\
\text { cultural/ historical contexts" (p. 95). }\end{array}$ \\
2 & $\begin{array}{l}\text { Recognition and encouragement for } \\
\text { utilization of multiple FoK. }\end{array}$ & $\begin{array}{l}\text { "Pedagogy as resistance to schooling hegemonies } \\
\text { (particularly to cultural deficit thinking) and as relationship } \\
\text { and interaction that promote a sense of humanity" (p. 96). } \\
\text { Focus on incorporation of FoK, rather than supplementary. }\end{array}$ \\
\hline
\end{tabular}


3 Process of classroom transformation involving teachers and students as learners and agents within and beyond the classroom.
"Pedagogy as micro- and macro-level consciousness and as conduit for personal, institutional, and societal transformation" (p. 98).

Focus on community cultural practices and heritage knowledge. Includes transformative learning related to "dark FoK" (Zipin, 2009, p. 320).

Theme 1 includes development of new curriculum units from themes from students' FoK, so that units of learning were "responsive to the interests of students and to the pedagogical objectives of teachers" (Browning-Aiken, 2005, p. 167). For example, Lee, a Hmong teacher, focused strongly on environmental science to highlight the relevance of Science examples to Hmong culture, and help Hmong students "look at Science not as a "difficult subject but a subject that was connected to their lives"' (Upadhyay, 2009, p. 224). Hensley (2005) drew on families' quilting traditions to help students learn about tessellations. Further examples of Theme 1 utilized dialogic inquiry to draw out students' FoK to scaffold their academic learning (Calabrese Barton \& Tan, 2009; Kumpulainen \& Lipponen, 2012).

As Amanti (2005) noted, such efforts were "not about replicating what students have learned at home, but about using students' knowledge and prior experiences as a scaffold for new learning" (p. 135). A further finding from her study was that learning about students' FoK was a valuable diagnostic assessment tool, thus helping her to avoid teaching concepts students have mastered, gaining time for advanced skill development. Students' subsequent schoolwork reflected benefits from activities that highlighted their FoK. For instance, after students "used shoeboxes to introduce themselves to their new Year 2 teacher" (Hughes \& Greenhough, 2006, p. 479), the teacher "was struck by the impact... on the children's creative writing. She talked about several children having 'literacy breakthroughs' as a result of this work" (Hughes \& Greenhough, 2006, p. 481).

Theme 2 applications primarily centered on students' FoK. Examples include: literature studies that drew on African American students' signifying expertise (Lee, 2001); cross-curricular units on horses linked to Latino students' shared interests and experience (Amanti, 2005) and local geology and mining (Browning-Aiken, 2005); a dream house construction unit (Ayers et al., 2001); a mathematics-science unit relating to a classroom garden (Kahn \& Civil, 2001); and writing a soap opera (Thomson \& Hall, 2006). Some Theme 2 pedagogical applications did double duty by enhancing academic knowledge and strengthening bonds within families. For example, oral history assignments helped students learn about family members and appreciate the value of their knowledge (Flores Carmona \& Delgado Bernal, 2012; 
Marshall \& Toohey, 2010; Olmedo, 1997). Camangian's (2010) use of students' auto-ethnographies also did double duty by enhancing the classroom ethic of care and informing students' critical analysis of society.

Patterson and Baldwin (2001) stated instructional changes involved "two simple shifts: more use of students' background knowledge; and more focus on relevance and student inquiry" (p. 133). Co-construction of learning related to students' FoK allowed them greater ownership (Bouillion \& Gomez, 2001; Sandoval-Taylor, 2005) and challenged school norms regarding acceptable topics (Pirbhai-Illich, 2010).

Theme 3 applications moved the focus beyond the classroom. Examples were authentic learning activities with real-life purposes (Bouillion \& Gomez, 2001; Marshall \& Toohey, 2010). For instance, on a neighbourhood walk elementary students identified their concern about the state of a local river, and this became the topic of their science investigation. Therefore, this authentic learning activity drew on students' FoK and related to their identities as citizens within the local community (Bouillion \& Gomez, 2001). Further examples include: engagement with Mien parents as experts to collectively build a Mien house and garden as part of a crosscultural Science unit (Hammond, 2001); student research into gangs and weapons to inform a video production (Pirbhia-Illich, 2010); and publishing picture books from grandparents' stories of earlier life in India (Marshall \& Toohey, 2010). Further benefits arose from students' growing identity as knowledge producers. Marshall and Toohey (2010) found students were highly motivated to "get the stories 'right,' not only for their eventual audience but also for the grandparent-tellers" (p. 226).

\section{NEW THINKING ABOUT STUDENTS, PARENTS, AND SELF}

Involvement in FoK research alerted teachers to a new way of knowing and interacting with students and parents. Teachers became more strategic in their relationships with parents, deliberately building rapport and finding out about their talents and work as a way to garner pedagogical ideas (Cremin et al., 2012; Hensley, 2005). Attention to students' questions led to a group of Haitian boys (who were building drums and learning traditional drumming rhythms) learning about physics concepts and academic language by recording and describing sound waves of various drum rhythms (Conant et al., 2001). Messing (2005) reported that one teacher stated:

She never lacks in ideas for class projects, or for assistance in the classroom, since she sees students as contributors to the curriculum. (p. 193)

Therefore, teachers' new awareness potentially allowed for ongoing improvement of understanding and development of relevant classroom practice. 
Teachers began to tap into parents' expertise, involving them in classroom learning and curriculum development (Hammond, 2001; Hensley, 2005). For instance, as part of a multicultural bread-making unit, Hensley (2005) described how she invited family members to teach the class how to make tortillas, resulting in a successful visit by a parent and two grandparents. Positive consequences included growth in students' pride and self-esteem from seeing the value teachers placed on their parents' knowledge. Hensley (2005) also describes how, at her invitation, one father (Jacob) wrote a musical for the class to perform. Apart from generating a successful, enjoyable learning experience for the class, this experience proved life-changing for Jacob. He discovered his passion for working with children, the value of his skills, the potential of his talent, and experienced empowerment, appreciation, and success. Jacob's participation at school continued to grow; he wrote another musical, and became an effective leader within the school parent-teacher association. Thus, involvement of parents from minoritized groups empowered them through greater and more balanced representation (Hensley, 2005).

Tuning in to thinking about FoK in relation to planning learning experiences also caused some teachers to reflect more consciously on their FoK and draw on these in teaching (Hensley, 2005).

\section{CHALLENGES}

Implementing classroom activities that drew on FoK was not without challenges. Two studies reported challenges arising from illumination of students' "dark" FoK (Zipin, 2009, p. 320). For instance, in the Creative Partnerships project, students worked with a professional playwright to develop "filmic word sketches of hypothetical families" (Thomson, Hall, \& Russell, 2006, p. 31). The project aimed to provide students "the opportunity to develop their potential, their creativity and imagination through sustainable partnerships with creative and cultural organizations" (Creative Partnerships Nottingham (n.d.), cited in Thomson et al., 2006 , p. 29). The following is an excerpt from the students' collective work, capturing its dark tone:

We see a girl getting ready to go out in the home of the CARTISES. Amy goes out with a boy called Rayorno, who is a gangsta and her mum and dad hate him. Her gran Roseanne loves her job and is very hard working but most nights she comes home at 1.30 or $2.00 \mathrm{am}$ and is always drunk. Amy is missing her friend Emilia.

Amy: She wrote me a letter that made me want to see her even more because she was PREGNANT with a little girl. It's great! I want a baby with Rayorno, yeh cool. Anyway see ya I am going to try and beg Rayorno for a baby. (Thomson et al., 2006, pp. 32-33) 
Although teenage pregnancy was common within the area, it was unclear what resources students used to develop their script: what was the relative influence of soap operas and real life experiences? Did the script expose "dark" FoK? (Zipin, 2009 , p. 320). This was an issue for the school principal, who saw the work as "something that would potentially bring offence to sections of their school community" (Thomson et al., 2006, p. 31). A further risk was damage to the school's reputation, "which, in a competitive educational market with declining population, could be dangerous" (Thomson et al., 2006, p. 35). The script generated discussion about issues arising from teaching staff's lack of involvement, and the project was shut down. Therefore, in that context, the aim to produce high quality art, "an important reservoir of ideas, comment, critique, and surprise" (Thomson et al., 2006, p. 40) was at odds with school managers' needs. A further challenge that compromised teachers' ability to integrate students' FoK into classroom experiences was national curriculum constraints (Thomson \& Hall, 2008). Other issues were experienced in the RPin project, which aimed to "make the community curricular" (Zipin et al., 2012, p. 183) (also see Section 2.6). Teachers were shocked and scared by students' "experience of darkly violent effects of poverty and racism" (Zipin et al., 2012, p. 185). Some teachers preferred to avoid this emotional work (Ovsienko \& Zipin, 2007).

Rogers et al. (2004) found low-achieving African American students tended to speak of themselves in terms of deficiencies and undervalue their own expertise. They needed support to identify their own FoK. Rogers et al. (2004) concluded "by definition (FoK) are acquired and thus often preconscious i.e., readily practiced but difficult to explain" (p. 180). Students initially did not expect their low-SES community to include experts, but finding expert chefs, athletes, housekeepers and others helped them name their own FoK.

Saubich and Esteban's (2011) Living Morocco Project promoted further reflection regarding challenges of applying FoK to classroom pedagogy. I noted that the six teaching units described were "largely based on the funds of knowledge that were identified" (p. 94) as held by a Moroccan family living in Catalonia, Spain, such as FoK regarding Morocco's animals and geography. The stated aim of the study was to identify the Moroccan students' "linguistic and cultural resources... in order to explore ways in which these... can be used to extend the sociolinguistic repertoires - including academic language - of all students" (Saubich \& Esteban, 2011, pp. 99100). For example, in one activity, students located Morocco and Catalonia on a map and drew routes between the two. In a second activity, students worked in pairs, with one reading an animal's description, and the other cutting and pasting 
features onto a drawing to match information from the reading. Learning objectives included identifying the location of Morocco and physical distance between Morocco and Catalonia, and identifying animals of Morocco. However, I wondered what academic learning resulted for the Moroccan students. Although the classroom activities successfully disrupted some deficit theorizing towards Moroccan families in Catalonia, Saubich and Esteban (2011) noted that it was challenging to shift teacher practice so that application of the FoK concept was "at the heart of teaching" (p. 99), equivalent to moving from Theme 1 to Theme 2, according to Rodriguez's (2013) typology of pedagogical applications.

A difference between Saubich and Esteban's (2011) approach and González's (1995) "funds of knowledge methodology" (p. 238) was the apparent absence of a study group. Therefore, this distinction seemed to uphold González et al.'s (2005) claim about the central role of teachers' study groups to support teachers in FoK work. Cremin et al. (2012) provided training in researcher skills and knowledge for teacher participants, but found that teachers still found it challenging to apply their learning to classroom pedagogical practice. Therefore, I wondered how can teacher preparation and collaboration support rigorous theoretical grounding, development, and description of pedagogical strategies that recognize, relate to, and reframe students' FoK to support academic learning?

Challenges also affected oral history projects. Translating and developing written texts from grandparents' audio-recorded stories was difficult when they were "more in the form of memories than a linear life story" (Marshall \& Toohey, 2010, p. 226). This was resolved by the teacher's decision to work within the picture book genre, with elementary students as the intended audience, leading to a reduction in the text-focus. In this study, use of multimodel resources (e.g., MP3 players) supported students, allowing collection of stories in grandparents' home languages and translation into printed English. Also, students' final picture books on CDs as well as in print became "hybrid semiotic resources for the school - and they provided representation of the usually invisible and seldom talked about issues at school such as historical events in India and religious conflict" (Marshall \& Toohey, 2010, p. 236).

Beyond adapting pedagogical practice, teachers gained new insights into their professional role. For instance, as a result of participation, Floyd Tenery (2005) reflected that mediation was a key element of the teaching role: mediation of roles of teacher and researcher; mediator between home and school; and mediating interpretations - developing awareness of "the multidimensionality of students and their families" (p. 128). Inherent challenges of enacting these roles related to 
navigating tensions between avoiding assumptions and recognizing commonalities (Amanti, 2005).

\subsection{Limitations of current research}

As well as reporting on benefits arising from FoK work, it is appropriate to present some findings concerning research methodology. All studies I found (except one) utilized qualitative research methodology, allowing in-depth description of research contexts and reporting of complex findings relating to beliefs, relationships, and professional practice. Data collected related to FoK as well as teacher practices and perceptions. However, I found no studies that investigated students' perceptions, highlighting a gap in current knowledge.

The only FoK research I found that utilized a quantitative methodology was RiosAguilar's (2010) analysis of links between Latino families' FoK and Latino students' academic and non-academic achievement.

Table 2.2 shows fourteen texts related to studies in which teachers have learned about students' and families' FoK through home visits, using the so-called 'funds of knowledge methodology" (González, 1995, p. 238). Therefore findings presented here relate largely to outcomes from this methodology, applied in kindergarten, elementary and middle schools. Thus, further research would deepen knowledge of valued outcomes arising from diverse methodologies, particularly for students in secondary schools.

In most studies, the chosen approach to learn about students' FoK seems to have been determined by the researcher(s). However, in the Home School Knowledge Exchange Project (Feiler, Greenhough, \& Winter, 2006; Hughes \& Pollard, 2006), elementary students, their parents, and teachers were consulted about possible ways to achieve two-way knowledge exchange between families and school. Also, in Calabrese Barton and Tan's (2009) study, middle school students provided ideas about ways that their Science teacher could draw on their FoK.

Single teacher participants report findings in sixteen texts (Amanti, 2005; Ayers et al., 2001; Browning-Aiken, 2005; Calabrese Barton \& Tan, 2009; Camangian, 2010; Conant et al., 2001; Gutiérrez, 2002; Hensley, 2005; Kahn \& Civil, 2001; Lee, 2001; Pirbhai-Illich, 2010; Rogers et al., 2004; Seiler, 2001; Sugarman, 2010; Upadhyay, $2005,2009)$. Therefore this raises the question of transferability of findings across other potential participant groups and settings. However, these texts make valuable contributions to the literature by providing rich description of the process of undertaking research into students' FoK, including challenges encountered, impacts 
on relationships, changes made to classroom practice, as well as personal testimony about significance of the work for teachers.

The range of settings and approaches also raises questions about possible limitations of this approach, such as its usefulness within secondary schools, which this study hoped to illuminate.

\subsection{Conclusion}

Evidence collected in diverse research settings identified rich FoK held by groups historically subjected to deficit theorizing. Therefore, I agree with others (such as Andrews \& Yee, 2006; Zipin et al., 2012) that there is a moral imperative for educationalists to learn about minoritized students' FoK as a first step to redress disparities in educational experience and outcomes. Consistent findings related to positive benefits for teachers affirm the promise of applying a FoK approach, to achieve more accurate understandings of students and families, improve teacherstudent relationships, and develop teachers' understanding and application of culturally relevant pedagogy. Rich data from qualitative studies provide detailed descriptions of factors that support achievement of valued outcomes (such as study groups), as well as factors that have challenged participants (such as complex communities), which are valuable to me as an emerging scholar in the field.

Findings of my narrative review highlight lack of research regarding application of FoK in New Zealand schools, and limited research in high school settings generally. Furthermore, thus far there has been a lack of attention to student perceptions related to teachers' learning about FoK, and limited research on selection of strategies for learning about high school students' FoK. Therefore, I argue this study will add valuable original knowledge to the field by examining perceptions of valued outcomes arising from application of the FoK concept in a New Zealand secondary school setting. New knowledge will illuminate perceptions of students, parents/caregivers and teachers regarding valued outcomes from teacher identification of students' FoK, preferred approaches for teachers to identify students' FoK, and factors that facilitate or hinder achievement of valued outcomes from this process. 


\section{Chapter 3: Methodology}

What's different now is that everyday life, including life in schools, seems to be faster paced, more diverse, more complicated, more entangled than before. The kinds of personal and social relationships, exchanges, and networks we participate in seem to be taking new forms, tying together otherwise disparate people, and demanding some new ways of thinking about what to research and how to do it. (Eisenhart, 2001, p. 24)

\subsection{Introduction}

Lincoln and Guba (2000) note recent trends to acknowledge benefits when paradigms "interbreed" (p. 164), as scholars recognize how different theories can "inform one another's arguments" (p. 164); reflecting this trend, the present study incorporated elements to create a "blend" (Gall, Gall, \& Borg, 2007, p. 490). The methodology was influenced by constructivist and emancipatory paradigms. This was appropriate because the study explored multiple participants' perspectives of applying the FoK concept in secondary schooling, to advance social justice. Similarly, this case study was enriched by ideas from proactive action research (Schmuck, 2009). In analysis, thematic coding was enhanced by Flick's (2006) approach to addressing data from multiple cases. This chapter aims to fully describe and explain the methodology, to demonstrate its suitability to address the research questions.

The chapter begins with discussion of the research aims and questions, and identifies the operational definitions. The suitability of a qualitative study, within the constructivist paradigm, and application of some principles from the emancipatory paradigm, are explained. The methodological choice of collective case study is justified, and details of key features of this case study are outlined, including research structure, researcher role, and sample. Next, four procedures are explained and justified: participant selection, participant recruitment, data sources, and data analysis. The penultimate section discusses ethical considerations, focusing on how ethical principles affected study design. The chapter ends with discussion of design features and procedures that enhance trustworthiness.

\subsection{Research aims and questions}

This study investigated application of the FoK concept (Moll et al., 1992) in a NZ high school. For the purpose of this study, FoK was defined as knowledge and skills developed from life experience outside formal schooling. 
The purposes of the study were to:

- increase high school teachers' knowledge of the FoK concept;

- explore use of collaboration between teachers, Māori and Pasifika students, and their parents;

- identify preferred strategies by which teachers can learn about students' FoK;

- develop understanding of multiple perspectives about the value and manageability of using the FoK concept within a high school;

- explore the value of this approach in relation to social justice aims.

The central research question was:

What valued outcomes arise from teacher identification of FoK of Māori and Pasifika students in NZ high schools?

Four sub-questions arose from this central focus:

1. What do participants perceive as the valued outcomes from teacher identification of ethnic minority students' FoK?

This question focused on multiple realities of participant groups (minoritized students, parents, and teachers) regarding valued outcomes from teacher identification of students' FoK. Participants constructed the key criterion variable (valued outcomes). Data collected identified participants' hopes regarding valued outcomes and perceptions of valued outcomes achieved.

2. What are the characteristics of approaches to teacher identification of ethnic minority students' FoK that generate valued outcomes?

The literature review showed diverse strategies to identify students' FoK, although few studies were conducted in high school settings. Studies by Norma González and Luis Moll, leading scholars in the field, largely focused on use of home visits, referred to as "the funds of knowledge methodology" (González, 1995, p. 238). In the present study, this question focused on identifying preferred strategies that teams in a NZ high school context agreed to implement for the teacher to learn about students' FoK. Data collection and analysis sought to identify reasons for strategy preferences, and features of selected strategies that participants perceived generated valued outcomes. 
3. What are the facilitators and/or barriers to achieving valued outcomes from teacher identification of ethnic minority students' FoK?

Analysis of literature revealed most FoK research in schools was conducted in elementary and middle school settings, raising the question of whether this concept may be usefully applied in high school contexts. This question sought to identify contextual factors that influenced achievement of valued outcomes. The research design included data collection for five teams of participants in a high school, enabling analysis of factors at various levels: school, team, participant and researcher. This question also allowed exploration of the potential of a team-based collaborative approach to identify preferred strategies by which teachers may learn about students' FoK, without the involvement of an external partner such as me. Therefore, this question relates to sustainability of a FoK approach in high school settings.

4. What do participants perceive as the significance of the valued outcomes?

This question focused on participants' perceptions about the worth of the approach under investigation.

\subsection{Operational definitions}

Key terms are funds of knowledge (FoK), and outcomes, defined thus:

Funds of knowledge (FoK): Knowledge and skills developed from life experience outside formal schooling.

Outcomes: Results, consequences, or effects.

For this study, the nature of valued outcomes was defined by individual participants, as suitable for a constructivist study with emancipatory aims.

\subsection{Research paradigm}

\section{Qualitative research}

The research was a qualitative study. The following discussion identifies some key features and interests of qualitative research, demonstrating the rationale for this approach.

Qualitative research can potentially "reveal and investigate the unique complexities and ambiguities of human subjects and their interaction in society" (Walliman, 2005, p. 247) and focus on "the importance of contextual and holistic understanding, with an emphasis on description, and a commitment to 'seeing through the eyes' of the people 
being studied" (p. 247). Therefore, qualitative research suits studies such as this that attempt to capture multiple perceptions of diverse participants (Denzin \& Lincoln, 2000). As Flick (2006) noted, qualitative research has high relevance in today's increasingly multicultural communities, with attendant "new diversity of milieus, subcultures, lifestyles, and ways of living" (Flick, 2006, p. 12). This criterion was relevant for investigating an approach's value for Māori and Pasifika high school students.

In qualitative research, the researcher is the main research tool (Janesick, 2003). This means the researcher's complex identity influences the study, affecting outcomes. Qualitative researchers must recognize and acknowledge they are "socially situated" (Denzin \& Lincoln, 2000, p. 19) by their "particular class, gender, racial, cultural and ethnic perspective" (p. 18). Thus, qualitative researchers need rigorous reflexive practices to examine their influence on the research - including research questions, data collected, interpretation, and theorizing from findings.

Because qualitative researchers focus on, "make sense of, or interpret, phenomena in terms of the meanings people bring to them" (Denzin \& Lincoln, 2000, p. 3), this approach aligns very closely with constructivism's epistemology (Gall et al., 2007). Thus, it suits investigations into complex phenomena, because contextual conditions are fully considered, and "objects under study are represented in their entirety in their everyday context" (Flick, 2006, p. 15). Qualitative researchers value "rich descriptions" (Denzin \& Lincoln, 2000), which provide an emic (insider) perspective of the investigated phenomenon (Bryman, 1988). Thus, qualitative research allowed me to gain insight into the experience and perceptions of Māori and Pasifika students, whose interests the study attempted to serve.

Also, qualitative research encompasses theoretical and methodological options, allowing alignment with the phenomenon under study (Flick, 2006). Close links between qualitative research and constructivism provided a clear pathway for the research design.

\section{Constructivist paradigm}

The study's ontology, epistemology and methodology shared significant features of the constructivist paradigm (Mertens, 2005). The research investigated valued outcomes resulting from teacher inquiry to identify students' FoK, as conceptualized by participants (minoritized students, parents/caregivers, and teachers). Participants constructed the key criterion variable (valued outcomes) during the course of the study. The research design anticipated variation in definitions of valued outcomes and developments in participants' ideas as the project progressed. Thus multiple perspectives were anticipated and valued. 
Epistemologically, researcher and participants functioned interactively within small group settings. Personal and professional values influencing the researcher were explicitly identified and described during planning (Mertens, 2005). Because the subject under investigation was value-laden, value-free objectivity was regarded as unrealistic (Guba \& Lincoln, 1989).

Methodologically, the study was highly contextualized, and qualitative in nature. Rich descriptive information regarding the context is provided. Qualitative methods included interviews, audio recordings of hui ${ }^{11}$, video recordings of team events to implement strategies, and reflective journal entries. As appropriate for a qualitative study in the constructivist paradigm, the research aimed to meet quality standards of credibility, transferability, dependability, and confirmability (Mertens, 2005) (see Section 3.10).

Mertens (2005) suggested in the constructivist paradigm, research questions evolve during the study. Accordingly, I was open to revising research questions if warranted, for instance, by participants' responses or recruitment issues. However, this need did not arise.

\section{Features of the emancipatory paradigm}

Some study features may be seen as aligned with the emancipatory paradigm (Mertens, 2005). In seeking minoritized student and caregiver participants, multiple realities from diverse standpoints were valued. The research aimed to empower student and parent participants who commonly have limited voice in decisions about teacher strategies. All participants collaboratively determined strategies for teachers to identify students' FoK with potential to lead to valued outcomes as defined by them. Also the study investigated participants' perceptions regarding significance of valued outcomes achieved.

Timing of fieldwork suited teacher and student participants' authentic working relationships. Fieldwork was conducted during one school year so each team remained relevant, due to ongoing teaching-learning relationships. This factor created a framework for participants' empowerment through any learning that might arise from involvement.

The study did not align fully with principles associated with the emancipatory paradigm, such as scrutiny of "illegitimate structural and interpersonal constraints" (Grundy, 1987) arising from societies' practices of repression and dominance, and the objective of system-level change (Zuber-Skerrit, 1996). Also, participants were not a "community of equals" (Cohen, Manion \& Morrison, 2007, p. 303) as I alone

11 Meeting. The Māori term hui is used for many research events, to show respect for Māori participants. 
determined the study focus. I argue that imposition of a topic focus was valid because the study explored FoK, which was not widely known in NZ schools. Also, application of FoK potentially could address concerns about teachers' knowledge of students and ability to support effective learning for minoritized students. As an academic, I had access to educational research findings of interest to participants. The study generated new knowledge for NZ high school teachers, with enhanced value from incorporation of parent/caregivers' and students' perspectives.

Although FoK was not widely known in NZ schools, I expected parents and students to have ideas about the value of students' knowledge and skills from life experience to schooling. I anticipated relating participants' FoK to academic concepts would elevate their status, and validate participants' wisdom and experiences.

Finally, in the literature review, I attempted to develop an overview of current relevant research, especially literature representing minoritized students' experiences, as appropriate for the emancipatory paradigm (Harding, 1993).

\subsection{Research method}

The present study utilized a collective case study approach (Stake, 2000). Following Yin (2009), Robson (2011) defined case study as "a strategy for doing research which involves an empirical investigation of a particular contemporary phenomenon within its real life context using multiple sources of evidence" (p. 136). Therefore, planning requires a clear vision of the phenomenon, case, focus, and unit of analysis (Gall et al., 2007).

Some case study researchers treat the case and unit of analysis as equivalent (Grunbaum, 2007), but I followed Grunbaum's typology of separating these elements, shown in Table 3.1.

Table 3.1: Key elements of this case study

\begin{tabular}{|c|c|c|}
\hline Element & $\begin{array}{l}\text { Definition } \\
\text { (Gall et al., 2007) }\end{array}$ & Application in this study \\
\hline Phenomenon & $\begin{array}{l}\text { "process, event, person, or other item of } \\
\text { interest to the researcher" (p. 447) }\end{array}$ & FoK \\
\hline Case & $\begin{array}{l}\text { "a particular instance of the } \\
\text { phenomenon" (p. 447) }\end{array}$ & $\begin{array}{l}\text { Application of the FoK concept by } \\
\text { teachers working with Māori and } \\
\text { Pasifika students in KHS, NZ }\end{array}$ \\
\hline Focus & $\begin{array}{l}\text { "the aspect, or aspects, of the case on } \\
\text { which data were collection analysis will } \\
\text { concentrate" (p. 448) }\end{array}$ & $\begin{array}{l}\text { Valued outcomes arising when teachers } \\
\text { learn about Māori and Pasifika students' } \\
\text { FoK }\end{array}$ \\
\hline Unit of analysis & $\begin{array}{l}\text { "the aspect of the phenomenon that will } \\
\text { be studied across one or more cases" } \\
\text { (p. 448) }\end{array}$ & $\begin{array}{l}\text { Valued outcomes } \\
\text { Factors which supported valued outcomes } \\
\text { Constraints to valued outcomes }\end{array}$ \\
\hline
\end{tabular}


Grunbaum clarified when conceptual distinction is helpful:

The unit of analysis is identical with the knowledge that key informants can provide the researcher with... After the collection of information the data analysis aims to facilitate a knowledge transformation... Hence the researcher needs to understand the case layers (i.e. the case) to be able to create a valuable knowledge transformation that is authentic and transferable (if that is a research goal). Each case layer is assumed to be on a higher level of abstraction than the previous. The case thus is unique and holistic. The case is interconnected in a non-causal way conveying that goals about producing generalizations are problematic if not impossible. (pp. 88-89)

Stake (2000) noted each case:

has working parts; it is purposive; it often has a self. It is an integrated system.... Its behavior is patterned. Coherence and sequence are prominent. (p. 436)

Therefore this research approach was highly suitable to illuminate influence of contextual factors (Robson, 2011). The single bounded system (Stake, 1995) of each case was a team, formed for the study. The structure of a typical team is illustrated in Figure 3.1. Recruitment aimed for teams that each included a teacher and student and parent participants associated with that teacher.

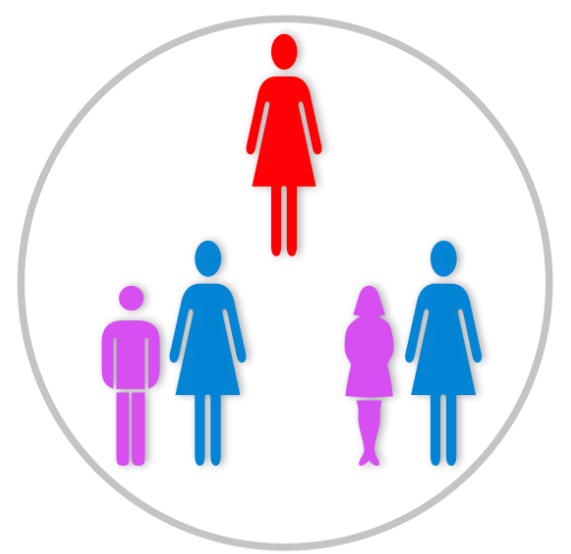

Figure 3.1: Example of a team

Figure 3.1 Key:

Participant type shown by color of figure

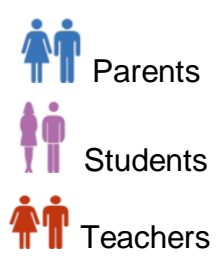

As illustrated in Figure 3.2, each team undertook an iterative process whereby members negotiated and agreed on strategies to implement, conducted implementation, and revised and evaluated both outcomes and process. (See Section 3.8 for description of research events and data collected.) 
Negotiation and implementation

of strategies for teacher to learn about students' FoK
Review and evaluation of outcomes from implemented strategies and team process

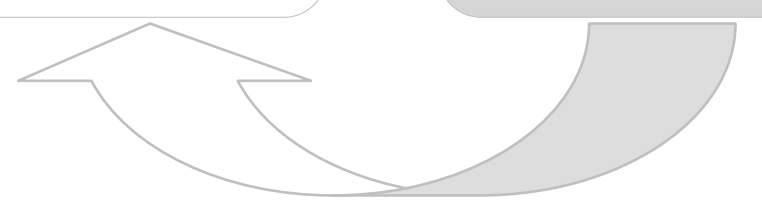

Figure 3.2: Cyclical process undertaken by teams

Multiple cases allowed an emic (insider) perspective of specific values and circumstances for different participants, and variables relevant to each bounded system, defined by working relationships within teams. Case study methodology allowed collection of rich data related to participants in each case, enabling detailed understanding of each case.

Because the research sought to explore applicability of a specific intervention in the context of a NZ high school, it was an instrumental case study (Stake, 2000), meaning the rich data relating to cases were collected to advance understanding of the issue under investigation. Because of the instrumental nature, my sampling method was to seek teacher volunteers, as recommended by Moll (González et al., 2005). When volunteer teachers formed the hub of each team (case), this provided the opportunity to learn what might be achieved with the approach under investigation, to illuminate how application of the FoK concept might improve effective teaching of Māori and Pasifika high school students.

Two reasons underpinned my decision to conduct cases simultaneously within one school community. Firstly, the research design was informed by Moll's recommendation that a teachers' study group performs an important mediating function when teachers learn about students' FoK. For González et al. (2005), study group settings were "the 'centre of gravity' of the project" (p. 17) (see Section 2.6). Study group settings deliberately applied sociocultural thinking and learning processes to help teachers work through their researcher roles, and make sense of information related to students' FoK.

In this study, study group sessions were called teachers' focus groups (TFGs). Two TFGs (TFG1 and TFG2) followed implementation of strategies from Hui 2 and Hui 3, and involved teachers in three main activities: Firstly, they considered background 
readings $^{12}$. Secondly, video footage relating to students' FoK was viewed and discussed. Two examples of video footage viewed were of students sharing personal profiles, and a student at Cook Islands performing arts group practice. Thirdly, teachers reflected on implications of new learning for their classroom practice.

Secondly, simultaneous conduct of cases enhanced manageability for me as the researcher and teachers. Because teacher participants were from one school, they formed a professional learning community within their school. Logistically, working simultaneously with teacher participants from one school helped avoid a very elongated period of data collection. Also, it was relatively manageable to get teachers from one school together for TFGs (avoiding additional scheduling challenges and commuting time).

My focus was on developing rich understanding of each case. However, a multiple case study design allowed cross-case analysis in order to theorize from findings, thus increasing new knowledge generated and allowing analytical generalization. Cases are not presented as typical, nor do they seek to provide an exemplar. In order to create new knowledge from the "complex, situated, problematic relationships" (Stake, 2000, p. 440) that make up the issue addressed within each case, development of understanding utilizes the research questions as a framework: What was achieved? What were the enabling factors? What were the barriers?

The cyclical process undertaken by each team has some alignment with characteristics of action research, identified by Schmuck (2009, p. 1): "focused on the goal of improvement, through the strategic planning change to achieve development, drawing heavily on the multiple perspectives of individuals and groups, to create improvements at a local level" (my italics). Additionally, as for proactive action research by an external researcher, this project applied democratic principles, seeking and valuing all participants' voices. Because I defined the project focus, the democratic form was participatory, not representative (Cohen et al., 2007). The main focus of investigation was not self-study, but identification and development of effective practice for participants within a high school community.

12 In TFG1 the reading was Spradley, J. P. \& McCurdy, D. W. (1972). The cultural experience: Ethnography in complex society. Palo Alto, CA: Science Research Associates Inc. pp. 13-17.

In TFG2 the reading was Lee. T. S. \& Quijada Cerecer, P. D. (2010). (Re)claiming native youth knowledge: Engaging in socio-culturally responsive teaching and relationships, Multicultural Perspectives, 12(4), 199-205. 


\subsection{Researcher role}

As researcher in a qualitative study, I was the primary research tool. In Chapter 1, my identity and subjectivity were fully explored, but aspects of my roles in data collection are briefly described here.

There were various aspects to my role as researcher. Firstly I facilitated research events throughout the project, beginning with teaching all participants about the FoK concept, and various approaches to teacher identification of students' FoK implemented in school settings internationally. Later I facilitated team hui in which participants negotiated preferred approaches for implementation by their team and reflected on valued outcomes, supports and barriers. Secondly, to understand how my beliefs and values were affecting the situation being investigated, I engaged in self-study. Reflective journaling was used to record impressions and questions, and develop ideas about how my "values, attitudes, perceptions, opinions, actions, feeding etc (were) feeding into the situation being studied" (Cohen et al., 2007, p. 310). (See Section 9.3.1.2 for discussion of unplanned aspects of how I enacted the researcher role.)

\subsection{Participants}

Participants were teachers, Māori and Pasifika students and their parents/caregivers from a NZ high school community, who were organized into five teams. Also, two school manager participants contributed contextual data related to the school. The school was selected purposively, to recruit enthusiastic teachers committed to working on a new intervention that aimed to support minoritized students. To assist manageability for me, the school was in the Wellington region.

Findings for each team are presented separately in Chapters 4-8. Each of these chapters begins with a description of the relevant team participants, thus introducing each team. The introduction focuses on the nature of the relationship between team members at the beginning of the study, and demographic information, including members' identity (teacher, student or parent), gender, and self-identified ethnicity. Figures provided illustrate participants within each team. For teams whose membership changed during fieldwork, two figures show participants at the start and end of the study.

To summarise the nature of participants involved in the teams, for the purposes of this chapter, information is presented in two tables. Table 3.2 provides an overview of all participants' demographic profiles, by team. Table 3.3 summarizes participants' demographic profiles by group. Finally, this section identifies participants' involvement 
in aspects of school life described earlier in Sections 1.4.2.2 and 1.4.2.5. Demographic data were not collected for school manager participants.

Some participants were involved in the school's whānau unit and/or Integrated Studies (IS) program ${ }^{13}$. Two members of Team B were involved in the whānau unit: Kate (teacher within the Māori faculty), and Dan (student). Georgia (Team C), also taught English in the whānau unit. The other six Māori student participants, from Teams A, B, C, D, and E were enrolled in mainstream classes. Students from Teams A, D, and $E$ were in classes involved in IS. During fieldwork, themes addressed by Year 9 IS classes were Diego Run (based on the novel of the same name by Deborah Ellis), and Extreme Beliefs.

Table 3.2: Overview of demographic profile of participants, by team

\begin{tabular}{|c|c|c|c|c|c|c|}
\hline & & Team A & Team B & Team C & Team D & Team E \\
\hline \multirow[t]{4}{*}{ Teacher } & Gender & Female & Female & Female & Male & Male \\
\hline & $\begin{array}{l}\text { Country of } \\
\text { birth }\end{array}$ & Britain & $\mathrm{NZ}$ & NZ & Britain & Britain \\
\hline & Ethnicity & Pākehā & Pākehā & Māori & Pākehā & Pākehā \\
\hline & Role & $\begin{array}{l}\text { Senior } \\
\text { manager }\end{array}$ & $\begin{array}{l}\text { Middle } \\
\text { manager }\end{array}$ & $\begin{array}{l}\text { Middle } \\
\text { manager }\end{array}$ & $\begin{array}{l}\text { Middle } \\
\text { manager }\end{array}$ & $\begin{array}{l}\text { Senior } \\
\text { manager }\end{array}$ \\
\hline \multirow[t]{3}{*}{ Students } & Gender & Two males & Two males & $\begin{array}{l}\text { One male \& } \\
\text { one female }\end{array}$ & Two males & Three males \\
\hline & Ethnicity & $\begin{array}{l}\text { C. I. Māori \& } \\
\text { Māori }\end{array}$ & Māori & $\begin{array}{l}\text { Māori \& } \\
\text { Samoan }\end{array}$ & Māori & $\begin{array}{l}\text { Māori, C. I. } \\
\text { Māori, \& } \\
\text { Greek }\end{array}$ \\
\hline & Year level & Year 9 & $\begin{array}{l}\text { Years } 12 \& \\
13\end{array}$ & $\begin{array}{l}\text { Years } 9 \& \\
11\end{array}$ & Year 9 & Year 9 \\
\hline \multirow[t]{2}{*}{ Parents } & Role & $\begin{array}{l}\text { One } \\
\text { stepfather }\end{array}$ & $\begin{array}{l}\text { Two } \\
\text { mothers }\end{array}$ & $\begin{array}{l}\text { Two } \\
\text { mothers }\end{array}$ & (none) & (none) \\
\hline & Ethnicity & Pākehā & Māori & Pākehā & (none) & (none) \\
\hline
\end{tabular}

Table 3.3: Overview of demographic profile of participants, by group

\begin{tabular}{|c|c|c|c|c|}
\hline & Gender & Ethnicity & Role & Country of birth \\
\hline Teachers & $\begin{array}{l}3 \text { females } \\
2 \text { males }\end{array}$ & $\begin{array}{l}4 \text { Pākehā } \\
1 \text { Māori }\end{array}$ & $\begin{array}{l}3 \text { middle managers } \\
2 \text { senior managers }\end{array}$ & $\begin{array}{l}2 \mathrm{NZ} \\
3 \text { Britain }\end{array}$ \\
\hline Students & $\begin{array}{l}1 \text { female } \\
10 \text { males }\end{array}$ & $\begin{array}{l}7 \text { Māori } \\
2 \text { C.I. Māori } \\
1 \text { Samoan } \\
1 \text { Greek }\end{array}$ & $\begin{array}{l}8 \text { Year } 9 \\
1 \text { Year } 11 \\
1 \text { Year } 12 \\
1 \text { Year } 13\end{array}$ & $11 \mathrm{NZ}$ \\
\hline Parents & $\begin{array}{l}4 \text { females } \\
1 \text { male }\end{array}$ & $\begin{array}{l}2 \text { Māori } \\
3 \text { Pākehā }\end{array}$ & $\begin{array}{l}4 \text { mothers } \\
1 \text { stepfather }\end{array}$ & (unknown) \\
\hline
\end{tabular}

13 See Section 1.4.2.2 
Teacher participants were involved in various TPL initiatives within the school. Kate and Georgia facilitated E Tipu E Rea TPL. Twenty volunteer teachers participated, including all five teacher participants in the present study. Georgia (as manager of the Improving Māori Achievement portfolio) observed faculty heads' classes to gather baseline data for learning leaders' classroom practice. Also as part of He Kākano in 2011, Anna and Georgia established a Māori Parents' Support Group with assistance from Māori parents engaged with the school recruiting others from their networks.

\subsubsection{Participant selection}

Snowball sampling (Cohen et al., 2007) was used to recruit other participants. The first participants recruited were high school teachers, who then identified students for potential involvement; finally, students' parents/caregivers were approached in the third snowball cycle.

González et al. (2005) recommend teacher participants in FoK research are volunteers, because of the time commitment involved. A second pre-requisite for teacher participants was the desire to work more effectively with Māori and/or Pasifika students. Thus, teacher participants were a purposive sample. I utilized an advisory group of five educational advisers ${ }^{14}$ to suggest potential participants, to reduce my own role in this process, providing an element of impartiality. I asked the advisory group to suggest potentially interested teachers, then identified schools from which two or more teachers were suggested. Thus, three potential school sites emerged; however, only one principal consented to participants within the school community being invited to participate. Ultimately, I hoped to identify six teachers, and eventually recruit 4-6 teachers, 8-12 students and 8-12 parent/caregivers. I anticipated some participant dropout, and hoped at least four teachers would remain involved throughout. The sample size was chosen for balance between sufficiency for collection of rich descriptive data (Stake, 1995) relating to research questions, and manageability for the researcher.

Purposive snowball sampling was appropriate for this study because it investigated valued outcomes of a teacher practice that was new to NZ high schools. Thus, in conducting this exploration with teachers who are willing and ready to try a new approach, findings reveal what can be achieved under these conditions. For this reason the teachers may be seen as the critical participants. Because the teacher participant sample was not representative of all NZ high school teachers, it would be inappropriate to draw inferences from findings for the profession in general.

\footnotetext{
14 I chose this group of advisers because several years ago I had an educational advisory role, so I have established professional relationships with this group of professionals, and I know that they work in a range of schools in the wider Wellington area.
} 


\subsubsection{Participant recruitment}

Participant recruitment was undertaken in two stages. Firstly, teacher participants were recruited. Each teacher nominated at least two student participants. I judged the teachers' control over this aspect to be important since teams would work closely over several school terms. Teachers were asked to nominate students who (1) identified as either Māori or Pasifika, (2) were members of his/her current class, which s/he was scheduled to teach for the entire school year, and (3) were likely to remain at the school until the end of the year. These were the only guidelines; teachers could apply any other criteria they considered desirable. The first guideline related to the research's social justice aim, and others arose to ensure authentic teacher-student relationships, providing a rationale for collaboration. I judged it important that teachers had some autonomy regarding whom to invite, and hoped this would enhance their comfort level and sense of ownership. (See Chapters 4-8 for teachers' reported rationale for student selection.)

The second recruitment stage related to student and parent/caregiver participants. I made personal contact with potential parent/caregiver participants to arrange individual meetings with family members. I invited all students and their families to meet at their preferred venues. At these meetings, potential participants had opportunities for discussion, and received written information and consent forms.

\subsection{Research procedures and data sources}

In this section the process is elaborated, and data collected in each event is identified. To summarize the process, as described earlier, participants were involved in an intervention in which they collaboratively negotiated an approach to teacher identification of ethnic minority students' FoK. The agreed approach was implemented and impacts identified through individual and collaborative reflection. These data informed planning for a second implementation cycle, followed by identification of impacts.

This process resulted in a number of research events, summarized in Table 3.4. I used the term research events as equivalent to Denzin's (1989) data methods, due to the nature of the study. Table 3.4 lists research events sequentially, identifying timeframe and data types collected at each. Table 3.4 also highlights relevance of data collected to each research question. Because of the number of research events, most were no longer than one hour long. Two exceptions were TFG1 and TFG2. 
Table 3.4: Data collection related to research questions

\begin{tabular}{|c|c|c|c|c|c|c|}
\hline $\begin{array}{l}\text { Timeframe } \\
\text { Note: } \\
\text { Total data } \\
\text { collection } \\
\text { timeframe was } \\
51 / 2 \text { months. }\end{array}$ & $\begin{array}{l}\text { Research } \\
\text { event }\end{array}$ & $\begin{array}{l}\text { Data types, in } \\
\text { chronological } \\
\quad \text { order }\end{array}$ & $\begin{array}{c}\text { What do } \\
\text { participants } \\
\text { perceive as } \\
\text { valued } \\
\text { outcomes? }\end{array}$ & $\begin{array}{c}\text { What are } \\
\text { characteristics } \\
\text { of approaches } \\
\text { that generate } \\
\text { valued } \\
\text { outcomes? }\end{array}$ & $\begin{array}{c}\text { What are } \\
\text { facilitators } \\
\text { and/or } \\
\text { barriers to } \\
\text { achieving } \\
\text { valued } \\
\text { outcomes? }\end{array}$ & $\begin{array}{c}\text { What do } \\
\text { participants } \\
\text { perceive as } \\
\text { significance } \\
\text { of valued } \\
\text { outcomes? }\end{array}$ \\
\hline \multirow[t]{3}{*}{ First $31 / 2$ weeks } & Hui 1 & Audio recording & $\checkmark$ & & & $\checkmark$ \\
\hline & & $\begin{array}{l}\text { Participants' } \\
\text { individual } \\
\text { reflections }\end{array}$ & $\checkmark$ & & & \\
\hline & & Questionnaire & $\checkmark$ & & & $\checkmark$ \\
\hline \multirow{2}{*}{$\begin{array}{l}\text { Immediately } \\
\text { following Hui 1, } \\
\text { over } 3 \text { week } \\
\text { period }\end{array}$} & Hui 2 & Audio recording & & $\checkmark$ & $\checkmark$ & \\
\hline & & $\begin{array}{l}\text { Written team } \\
\text { agreement }\end{array}$ & & $\checkmark$ & & \\
\hline $\begin{array}{l}\text { Following Hui } 2 \\
\text { and Hui } 3^{15}\end{array}$ & $\begin{array}{l}\text { Team } \\
\text { events }\end{array}$ & Video recording & & $\checkmark$ & $\checkmark$ & \\
\hline \multirow{4}{*}{$\begin{array}{l}2 \text { months after } \\
\text { Hui } 2 \text {, over } 21 / 2 \\
\text { week period }\end{array}$} & Hui 3 & $\begin{array}{l}\text { Audio recording, } \\
\text { transcription }\end{array}$ & & $\checkmark$ & $\checkmark$ & \\
\hline & & $\begin{array}{l}\text { Participants' } \\
\text { written } \\
\text { brainstorm } \\
\text { sheets }\end{array}$ & & $\checkmark$ & $\checkmark$ & \\
\hline & & $\begin{array}{l}\text { Written team } \\
\text { agreement }\end{array}$ & & $\checkmark$ & & \\
\hline & & Student work & $\checkmark$ & $\checkmark$ & & $\checkmark$ \\
\hline $\begin{array}{l}\text { PFGs and } \\
\text { SFGs: After Hui } \\
2 \text { and Hui } 4\end{array}$ & $\begin{array}{l}\text { Focus } \\
\text { groups }\end{array}$ & $\begin{array}{l}\text { Photographs of } \\
\text { summary on } \\
\text { whiteboard }\end{array}$ & & & $\checkmark$ & $\checkmark$ \\
\hline \multirow{2}{*}{$\begin{array}{l}\text { TFGs: after } \\
\text { team events } \\
\text { and Hui } 4\end{array}$} & & Audio recording & & & $\checkmark$ & $\checkmark$ \\
\hline & & $\begin{array}{l}\text { Participants' } \\
\text { written notes }\end{array}$ & $\checkmark$ & $\checkmark$ & $\checkmark$ & \\
\hline \multirow{3}{*}{$\begin{array}{l}21 / 2 \text { months } \\
\text { after Hui } 3 \text {, over } \\
3 \text { week period }\end{array}$} & Hui 4 & $\begin{array}{l}\text { Audio recording, } \\
\text { transcription }\end{array}$ & $\checkmark$ & $\checkmark$ & $\checkmark$ & $\checkmark$ \\
\hline & & Audio recording & & $\checkmark$ & & $\checkmark$ \\
\hline & & $\begin{array}{l}\text { Participants' } \\
\text { written } \\
\text { brainstorm } \\
\text { sheets }\end{array}$ & & & & \\
\hline $\begin{array}{l}\text { After Hui } 4 \text {, over } \\
2 \text { week period }\end{array}$ & $\begin{array}{l}\text { Individual } \\
\text { interviews }\end{array}$ & $\begin{array}{l}\text { Audio recording, } \\
\text { transcription }\end{array}$ & & $\checkmark$ & $\checkmark$ & $\checkmark$ \\
\hline
\end{tabular}

\subsubsection{Hui}

HUI 1

Hui 1 was an introductory session to develop participants' understanding of the FoK concept and approaches reported in research for teachers to learn about students'

15 Teams implemented different strategies, with diverse timeframes and timing. (See Section 9.2.2.) 
FoK. Thus, the purpose of Hui 1 was to prepare participants for later parts of the research project. I conducted Hui 1 seven times, to allow all participants to attend at a convenient time; only one participant did not attend. Different occurrences were attended by different groupings; exceptionally, Team E attended as a team.

All Hui 1 iterations were audio-recorded. Other forms of data collected were:

- Individual reflections, in which each participant recorded his/her hopes for valued outcomes from teacher learning about the students' FoK. Participants chose various methods of recording, including writing, audio-recording, and video-recording.

- Questionnaires, including items related to individual demographic information and relationships between team members prior to fieldwork.

HUI 2

Hui 2 was the first research event conducted in teams, with the exception of Team $E$. The purpose was discussion of possible strategies by which the teacher could learn about the students' FoK, and agreement about strategy(s) to implement. All Hui 2 events were audio-recorded. Other data collected were participants' written notes showing their ideas about preferred and disliked strategies.

After Hui 2, a follow-up email to the teacher provided a copy of the team agreement, and offered my support to implement agreed strategies, should they wish it. Data collected were the email and pdf file.

HUI 3

Hui 3 was a team event scheduled after implementation of agreed strategies. Each team discussed implementation, including perceptions about a strategy's manageability and usefulness, barriers and supports affecting implementation, and perceptions of impacts arising. Also, teams considered what they had done and achieved, and agreed on next steps. Most teams decided to implement a further strategy. All Hui 3 events were audio-recorded and transcribed. Other data collected were participants' written brainstorm sheets showing their perceptions related to implemented strategy(s).

HUI 4

Hui 4 was the final event for teams, scheduled after implementation of the second round of strategies. Each team discussed strategies they implemented, how it went, and perceptions of impacts. Teams also made recommendations arising from their experience. All Hui 4 events were audio-recorded and transcribed. Other data 
collected included participants' written brainstorm sheets, showing their perceptions of implemented strategies.

\subsubsection{Team events}

Team events were occasions when team members implemented agreed strategies. Some of these took the form of team meetings (TMs). For instance, Team A had three TMs: two TMs where members shared personal profiles, and one in which students co-constructed a unit plan with the teacher. Other types of team events included observation of students and application of strategies in classes. I was present at most team events, and video-recorded the events. Some team events also resulted in collection of other data, such as photographs of artefacts used in personal profiles (Team A, TM1) and whiteboard notes (Team A, TM2), and copies of student work (Team C).

\subsubsection{Focus groups (FGs)}

FG interviews played a dual role. Firstly, they facilitated discussion among participants, promoted deeper thinking and idea development, and achieved higher levels of sharing. FGs are useful because more ideas tend to develop in group interviews (Cohen et al., 2007), and agreement and disagreement between participants is elucidated (Arksey \& Knight, 1999). Interviewer questioning probed for meaning. Secondly FGs were interpretive, thus providing a collective member check of data and initial analysis, in an attempt to minimize potential misunderstandings from my unconscious communicative hegemony (Briggs, 1986). Venues and times were negotiated with participant groups for their safety and convenience.

Three FGs were established, one for each participant group i.e., students (SFGs), parents (PFGs), and teachers (TFGs). Firstly I will describe SFGs and PFGs. TFGs are discussed separately because they had a different function.

SFGs and PFGs were held at two stages of the research. The first round comprised two PFGs (PFG1 and PFG2) and two SFGs (SFG1 and SFG2), which all occurred shortly after Hui 2. In the first round, data were collected relating to participants' perceptions of their experience negotiating in their teams, their feelings and ideas about agreed strategies, and hopes related to valued outcomes. Two FGs were held for student and parent participants to provide a choice of times for participants' convenience.

The second round of FGs (PFG3 and SFG3), was scheduled after Hui 4. Data collected related to participants' perceptions of their experience within teams, feelings and ideas about agreed strategies, and valued outcomes arising from implementation. 
Three TFGs were held. The first two (TFG1 and TFG2) functioned as a study group setting (see Section 3.5). They were the only research events that exceeded one hour: TFG1 was three hours long, and TFG2 was 90 minutes long. TFG3, held after Hui 4, had the same function as SFGs and PFGs. TFGs were fully attended by teacher participants, apart from one teacher who was absent from TG3.

Data collected in FGs included audio-recordings and photographs. Final FGs (i.e., SFG3, PFG3, and TFG3) were transcribed.

\subsubsection{Individual interviews}

All participants attended an individual interview, scheduled as the final data collection event. Five students took up the option of a group interview: the two Team D students were interviewed as a pair, and attended two interviews, and the three Team $\mathrm{E}$ students were interviewed as a group.

Interviews were important to allow expression of personal experiences and individual ideas. Non-directive interviewing (Cohen et al., 2007) gave participants "freedom ... to express her subjective feelings as fully and as spontaneously as she chooses or is able" (Cohen et al., 2007, p. 356). All interviews were audio-recorded and transcribed. Summaries were returned to participants for verification or correction.

\subsubsection{Data analysis}

The main approach to data analysis was thematic coding analysis (Robson, 2011). As well, I drew on ideas from thematic analysis (Flick, 2006) and structural analysis (Gall et al., 2007), drawing on Peräkylä's (2005) argument that applying several approaches to analysis can "let their results cross-illuminate" (p. 2005).

\section{THEMATIC CODING ANALYSIS}

Features of thematic coding (Robson, 2011) which appealed to me were that: it is suitable for collective case study, because it is applicable to a range of research methodologies; it provides a credible, principled approach to analysis of large quantities of data; and it is recommended for beginning researchers such as myself (Robson, 2011). The following paragraphs describe phases in thematic coding, and what comprised each phase. Although four phases are distinguishable, in practice I moved between phases as new insights arising from a case prompted my return to cases already examined to rethink my analysis. For example, a theme that arose inductively from Team A was that of inclusive team actions, and discussion with my supervisors highlighted the need to revisit Team $D$ data to look for instances within 
that case. In other words, I needed to make sure I was not missing possible themes due to other interpretations becoming unduly prominent in my thinking.

Beginning with the first phase, familiarization with data collected, data analysis occurred throughout the project (Stainback \& Stainback, 1988). This consisted of my own checks and reflection on data gathered, collaborative checking and reflection with participants, and data storage management.

Methods I used to reflect during data collection included recording audio-taped reflections, writing journal entries, and adding notes and questions arising from data. Three illustrative examples of notes and questions which I recorded on the transcript of Team A's Hui 3 are provided.

Firstly, reading my longwinded opening remarks to Team $A$ about the purpose of the Hui 3, I wrote "How hard must it be to listen to a long speech like this!!" Ongoing reflection on my verbal and non-verbal contributions was important for me as a beginning researcher; I was constantly looking for ways to improve my clarity and conciseness, while still providing enough time and space to allow participants to think and respond. Secondly, when Team A members talked about the experience of delivering their personal presentations, Sonny Bill said Lizzie had "more life experience" (Team A Hui 3, line 289). Wondering how hard it might be for students to see their own areas of expertise, I recorded the question: "Did Sonny Bill feel he had enough to talk about?" Thirdly, beside Lizzie's comment about what she learned from the personal profiles, I wrote "Yes, Lizzie is saying she felt the profiles showed people's identity, but not specifically their FoK, but can people identify these easily? Or is it something that we need to learn how to glean from what they say and how they say it?" Although I undertook to review and notate transcripts on an ongoing basis, this was not always manageable during intensive data collection periods, such as one week when I completed 17 interviews. As well, repeated listening to recorded data was necessary to check and correct transcriptions. However, due to time constraints, when data collection events were summarized, I relied on audiorecordings to provide greater detail.

Ongoing reflection and impressions were shared with participants. During data collection, some data were collected on templates. During interpretive FGs ideas were recorded on a whiteboard and verified /adjusted by participants. Summaries of data collected were also checked by participants.

After data collection was completed, I revisited data storage to organize hard copies of data according to case, in preparation for full immersion in each case during 
deep analysis. Electronic data were organized by data collection method (e.g., hui 3, interviews), and within each of these, by case. FGs provided cross-case data.

Phase two of thematic coding analysis was development and application of codes, which I undertook using NVivo 8 software. (Fuller discussion of processes used within NVivo is presented separately later in this section.) In phase two, all collected data were read and coded in recognition of their potential value. Coding divided data into categories for the purpose of identifying and understanding participants' ideas and experiences. Thus, data were divided into meaning units, each conveying specific meaning. The size of meaning units varied from conversations to short phrases, because the key characteristic was meaning conveyed. Some data were coded in more than one category. Because all data were coded, codes captured a range of different elements. These included: behaviors (e.g., responding, silence), interaction (e.g., speaking for students, inclusive team actions), participation (e.g., speaking freely, things unsaid), constraints (e.g., I just keep forgetting), activities (e.g., new armour to pedagogy, looking with a different intent), and meanings (e.g., commitment, you needed good questioning skills, strategies were fun).

Analysis of some data did not utilize NVivo, such as data from video-recorded TMs, data that were audio-recorded but not transcribed ${ }^{16}$, and photographs taken in FGs.

A mixture of deductive and inductive coding was applied, with pre-planned categories aligned to research questions and further categories generated as data review revealed other emergent factors. Because I planned some deductive coding, I began by creating nodes ${ }^{17}$ directly related to research questions. Within these parent nodes, child nodes emerged inductively as I trawled again and again through data, and I often drew on participants' own words to name child nodes. The following examples are provided for illustration. Since the unit of analysis was valued outcomes, with a focus on investigating whether valued outcomes for which participants hoped were achieved, I deductively created a parent node called hopes. Later, inductive analysis resulted in child nodes such as new armour to pedagogy hope, talk to me hope, teacher-student relationship hope. I also established a parallel node for outcomes, including a child node for each expressed hope, to enable analysis of hope fulfillment. Other nodes emerging inductively related to features of team hui processes (e.g., building relationships, preferences, responses, and silence).

16 Not all data were transcribed because of funding constraints. e.g., SFG1 and SFG2.

17 Within NVivo, categories are called nodes. The concept of sub-categorization is captured by the terms parent node (broad category) and child node (sub-category within the parent node). 
As I developed nodes within NVivo, I simultaneously built a node summary table in a Word document, to record each tree node's structure, and the exact nature of grandparent, parent and child nodes. I copied and pasted all node names and descriptions from NVivo to the table as I worked. Different colored shading within the table showed child nodes from the same parent, giving a visual image of separate nodes and children within each. I regularly re-evaluated nodes, sometimes making adjustments. For instance, some nodes were merged and for some, descriptions became more extensive. I kept different versions of the table, providing an audit trail of my developing coding categories. Table 3.5 shows an extract from the node summary table.

The third phase of thematic coding was theme generation, followed by "constructing thematic networks" (Robson, 2011, p. 476). During data collection and analysis, journaling helped me keep track of my ideas about potentially relevant themes. Some techniques I found helpful to identify themes were frequent recurrences of a concept, participants' own words, and theory from literature (Robson, 2011). Later, following coding for a case, a graphic overview was constructed to reveal links between coded items and overarching themes, and check for completeness.

Fourthly, after identification of themes, display techniques drew these together meaningfully, illustrating similarities and comparisons within and across themes (Robson, 2011). Data display greatly assisted data reduction and selection processes (Miles \& Huberman, 1984). This phase focused on "exploring, describing, summarizing and interpreting the patterns" (Robson, 2011, p. 476). For example, Figure 3.4 shows the graphic overview that summarizes and draws links between themes from Team D.

Finally, phase five was the culmination of earlier steps, in which cross-theme analysis draws out essential meaning regarding "what the data are telling you" (Robson, 2011, p. 483). To develop this overview, I engaged with the data set as a whole, and took time to reflect on patterns and trends, note frequently occurring items, look for relevant similarities and differences, and search for intervening variables which could explain connections between observed factors (Robson, 2011). Some useful networks to help generate meaning of data were: event flow networks; hierarchical presentation of grandparent, parent and child nodes; and causal networks to show links between variables. 
Table 3.5: Node summary table (extract)

\begin{tabular}{|c|c|c|c|}
\hline Parent node & Description & Children & Description \\
\hline \multirow[t]{5}{*}{$\begin{array}{l}\text { Teachers' } \\
\text { meaning- } \\
\text { making }\end{array}$} & $\begin{array}{l}\text { Items which relate to } \\
\text { conceptualization and } \\
\text { theorizing about FoK }\end{array}$ & $\begin{array}{l}\text { Connecting to } \\
\text { teachers' prior } \\
\text { knowledge }\end{array}$ & $\begin{array}{l}\text { Items which relate to teachers' } \\
\text { previous training, knowledge, and } \\
\text { beliefs, and how the principles in } \\
\text { this study relate to that }\end{array}$ \\
\hline & & Ideas about students & $\begin{array}{l}\text { Comments about students e.g., how } \\
\text { motivated s/he is to learn, } \\
\text { personality traits }\end{array}$ \\
\hline & & What teachers noticed & $\begin{array}{l}\text { Comments and observations made } \\
\text { by teachers when viewing data } \\
\text { collected relating to students' FoK }\end{array}$ \\
\hline & & $\begin{array}{l}\text { You needed good } \\
\text { questioning skills }\end{array}$ & $\begin{array}{l}\text { Teachers' observation and ideas } \\
\text { about skills to draw out students' FoK }\end{array}$ \\
\hline & & Teachers' self-analysis & $\begin{array}{l}\text { Comments made by teachers about } \\
\text { his/her own self e.g., personality } \\
\text { traits, skills, challenges, } \\
\text { weaknesses, strengths, pedagogical } \\
\text { techniques }\end{array}$ \\
\hline \multirow[t]{5}{*}{$\begin{array}{l}\text { Outcomes } \\
\text { arising }\end{array}$} & $\begin{array}{l}\text { Participants' } \\
\text { perceptions of } \\
\text { impacts arising from } \\
\text { the strategy } \\
\text { implemented, } \\
\text { including what was } \\
\text { learned about the } \\
\text { students }\end{array}$ & T-S Relationship & $\begin{array}{l}\text { Improved relationship between } \\
\text { teacher and student }\end{array}$ \\
\hline & & Enjoying school & $\begin{array}{l}\text { Students enjoying school, happier in } \\
\text { class, remaining at school }\end{array}$ \\
\hline & & $\begin{array}{l}\text { New armour to } \\
\text { pedagogy }\end{array}$ & $\begin{array}{l}\text { Teacher practice, including } \\
\text { developments in teacher's ideas } \\
\text { about teaching and learning } \\
\text { strategies, to incorporate ideas } \\
\text { and/or actions which identify or } \\
\text { draw on students' FoK }\end{array}$ \\
\hline & & $\begin{array}{l}\text { Knowledge of the } \\
\text { students }\end{array}$ & $\begin{array}{l}\text { Teacher perceptions related to } \\
\text { his/her learning about the students' } \\
\text { FoK }\end{array}$ \\
\hline & & Talk to me & $\begin{array}{l}\text { Participants' perceptions of having } \\
\text { attention paid to them by the teacher } \\
\text { e.g., engage with them in a personal } \\
\text { conversation, acknowledge students' } \\
\text { interest or achievements, ask about or } \\
\text { talk with them about a recent event of } \\
\text { interest }\end{array}$ \\
\hline
\end{tabular}

\section{STRUCTURAL ANALYSIS}

Some elements identified in data analysis may be seen as indicative of structural analysis (Gall et al., 2007) concerned with identifying "patterns inherent in the data" (p. 569, authors' italics). Patterns of interest included how participants responded to each other's suggestions and responses when participants shared personal information. This analysis helped me identify data that explained 


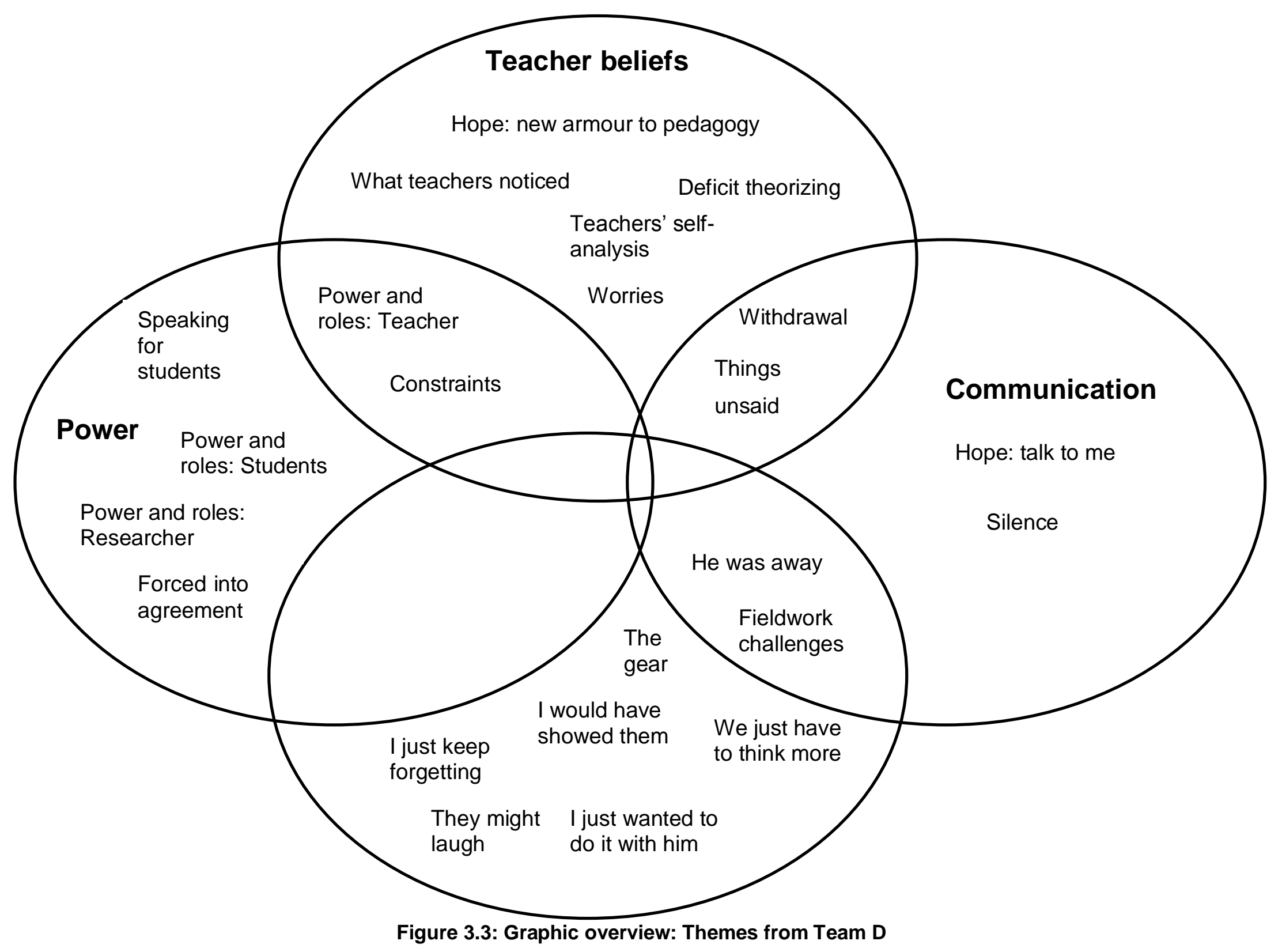


participants' perceptions of their experience, providing an evidential basis for theory development. I would argue that this analysis, although focused on particular types of data, is appropriate for this study within the framework of analysis tools utilized, because it facilitated identification and exploration of themes to address the research questions.

\section{THEMATIC ANALYSIS}

The sequence of post-data collection analysis was also influenced by ideas from thematic analysis (Flick, 2006), which he argues is suitable:

For comparative studies in which the groups under study are derived from the research question and thus defined a priori. The research issue is the social distribution of perspectives on a phenomenon or a process. The underlying assumption is that in different social worlds or groups, differing views can be found. (p. 307)

Thus, this approach has relevance for studies such as this in which multiple perspectives of participants have a key role. Also, Flick's (2006) description of the a priori nature of groups under study resonated with my use of teams constructed for the study. Flick notes that in such studies, "the collection of data is correspondingly conducted with a method which seeks to guarantee comparability by defining topics, and at the same time, remaining open to the views related to them" (p. 307). As noted earlier, the potential of this analysis method to help me develop rich understanding of each case, generate meaning from cases, and support theory development from examination of cross-case themes appealed to me.

Therefore, after data collection and initial familiarization with data (according to phase one of the thematic coding approach) were completed, I applied the following sequence of work:

1. Preparation of a short description of each case, including information about participants and summarizing findings (subsequently incorporated into reporting of results, in Chapters 4-8);

2. Deep analysis of a single case (Team D), including development and application of coding categories, looking for links between coded items to identify themes, and comparing and contrasting within and across themes (as for phases two to five of the thematic coding process);

3. Deep analysis of other cases in turn, for Teams $A, B, E$, and $C$ respectively, with cross-check of categories and thematic domains;

4. Display of results by case, showing similarities and differences related to relevant topics within the study;

5. Comparison of cases using the thematic structure developed. 


\section{USE OF SOFTWARE}

NVivo software was utilized to store data, and develop coding to categorize data. NVivo eased the task of sorting categories as more data was analyzed (Mertens, 2005). It also safeguarded work by allowing backups.

All transcribed data were imported into NVivo for coding, including interviews, Hui 3 and Hui 4 for each team, hopes recorded by each participant, email communication between myself and participants, team agreements, participants' written notes from Hui 2, 3, and 4, audio-recorded researcher reflections, and FGs held at the end of the study. Also imported were researcher journal entries, notes relating to data that were not transcribed, and earlier FGs.

Other data, such as video footage of team events, and photographs, were also incorporated as part of the analysis process. Budget constraints prevented transcription of all collected data; however, the amount of transcribed data may have been overwhelming.

I placed all nodes in the tree node area, to allow me to build coding categories and sub-categories (known as parents and children). This strategy gave me the flexibility to add sub-categories throughout, and enhanced the manageability of the coding process.

MindNode Pro and Microsoft Word software were used to build mind-maps of emerging patterns and concepts. Although NVivo offers these functions, I chose other softwares I had previously used for mind-mapping.

\subsection{Ethics}

Ethical principles were paramount in planning the research, as evidenced by approval granted by the Victoria University of Wellington Faculty of Education Ethics Committee, secured before commencement. (See Appendix A). In this section I describe how ethical principles informed research design and procedures. The section is organized according to ethical principles. (See Section 9.3 for ethical issues that arose in fieldwork.)

\subsubsection{Voluntary informed consent}

My decisions and actions relating to recruitment were underscored by principles of voluntary participation and informed consent. Information sheets were clear, comprehensive, and used language that was understandable for participants. They described the research purpose and questions, my personal background, what 
participation would entail, participants' rights, and how they would be protected. Consenting participants had the right to withdraw up until Hui 4.

In gaining participants' informed consent, as well as providing information sheets, I made a short DVD video to make the study more understandable. For students the youngest, most vulnerable participants - the consent process offered two student recruitment meetings, and opportunities for other discussions with me, to allow sufficient reflection opportunities.

Student participants, who were between 12 and 18 years of age, and members of minoritized ethnic groups, were the most vulnerable participants. Therefore, as well as his/her own consent, parental consent was also required for participation. This was also important because participation would span several months.

During fieldwork, I was mindful that participants might find it difficult to express their wish to withdraw, because of my status as a university researcher. To combat this risk, I remained alert to this possibility, and sought ongoing consent, by reminding participants of their right to withdraw when their actions seemed to suggest this might be their wish (see Section 9.3).

\subsubsection{Non-maleficence and beneficence}

Both non-maleficence and beneficence are concerned with impacts of the study on participants. Murphy and Dingwall define the two principles as follows:

Non-maleficence: researchers should avoid harming participants

Beneficence: Research on human subjects should produce some positive and identifiable benefit rather than simply be carried out for its own sake. (Murphy \& Dingwall, cited in Flick, 2006, p. 46)

The study applied the principle of confidentiality in order to protect participants from possible harm, if results could have been "harmful to the(ir) reputation, dignity, or privacy" (Walliman, 2005, p. 348). All participants chose a pseudonym, and I allocated a pseudonym to the school and other individuals named in data collected. Participants were informed of their right to confidentiality, and of their responsibility to maintain confidentiality within the team. Other professionals with access to data (e.g., transcribers and a peer reviewer) signed confidentiality agreements. Hard copies of data were stored in my locked office or in locked cupboards at my home. Electronic data were kept secure on my password-protected personal laptop, work computer, and a hard drive stored in a locked cupboard at my home. Data will be securely destroyed five years after the study's completion. 
There is a danger of losing some meaning from collected data in transcription and interpretation processes, potentially creating further participation risks. Indeed, I discovered retrospectively some transcribers summarized some elements in audiorecordings, judging them to be irrelevant. In order to capture as much meaning from data collection as possible, I regularly completed reflections and journal entries after data collection events, annotated transcribed material with comments and questions, and used member checking processes. Complementing my own efforts, my supervisors also posed questions, helping me to be reflexive about my "own cultural assumptions, theoretical stance and personal attitudes, and knowledge of other ways that the analysis can be approached" (Walliman, 2005, p. 352).

A number of opportunities provided gave participants access to findings. Interim findings were reported to participants as part of a celebration to mark the end of fieldwork. At their request, I met Team B participants to share the poster prepared for the 2013 AERA Annual Meeting, related to participants' experiences of the collaborative team approach. As promised during negotiation of entry to the site, I made myself available to present findings to KHS staff. However, the principal informed me the school avoided TPL events that took the approach of delivering knowledge to staff. Therefore, it seemed I would not be taken up on this offer.

However, the two principles of confidentiality and credit are incompatible, meaning participants could not collaboratively report findings with me or gain public recognition for their work.

\subsubsection{Reciprocity}

The research design aimed to provide reciprocity for participants, so benefits of involvement significantly outweigh costs (Frankfort-Nachmias \& Nachmias, 1992, cited by Cohen et al., 2007, p. 51). As pointed out by L. T. Smith (2005), this issue has particular significance for Māori, who have historically suffered from violations of this principle. Participants attended at least seven events ${ }^{18}$, and I showed appreciation of their ongoing commitment by offering manakitanga ${ }^{19}$. This meant I catered each research event generously, with fruit, home baking, and drinks. In Māori culture, provision of manaakitanga is very important as a show of respect, as shown by the whakatauki 20 "Tangata takahi manuhiri, he marae puehu:" someone who disregards visitors will find they have none (Kohere, 1997). Similar values are

18 Participating students and parents attended four hui, two FGs, and one individual interview, as well as any events which were agreed by the team. Participating teachers also attended the aforementioned events, as well as an additional FG.

19 Manaakitanga is a Māori term which encompasses ideas related to respect, hospitality, nurturing, and kindness.

20 Whakataukī is a Māori term which means proverb. 
held within both $\mathrm{Cl}$ Māori and Samoan cultures of some student participants. Therefore, in this context, attention to manaakitanga was an important aspect of research procedures.

A collective celebration of project completion also enhanced reciprocity. The first element of the closing celebration was a half-day tour of the university campus for interested students, as requested by a student during recruitment. Seven of the eleven student participants attended the tour. The second element, afterwards, was a formal thank you and meal for all participants on the university campus. Dan (Team B) opened the formal ceremony in Te Reo Māori ${ }^{21}$, and I thanked all participants in a short speech ${ }^{22}$ and presented interim findings. Finally, we shared a meal I had prepared. This was important in order to respect and honour participants' commitment and bonds within teams. Also, I had developed relationships with participants, and I wanted to exit the school site in a way that was sensitive to these new relationships.

\subsection{Trustworthiness of findings}

Guba and Lincoln (1989) argue four criteria are paramount for evaluating qualitative research: credibility, transferability, dependability, and confirmability. In this section, the meaning of each criterion and my associated processes are discussed.

\subsubsection{Credibility}

Mertens (2005) identifies four components of credibility. The study was designed to consider each of these.

\section{PROLONGED ENGAGEMENT IN THE FIELD}

Both corroboration and coherence were enhanced by sustained engagement in the field, as evidenced by Table 3.6, a graphical presentation of days on which fieldwork was undertaken. Because data were collected over six months, this reduced the risk of fleeting situations gaining undue prominence, and allowed observation of longterm trends. Also, my presence as a participant observer in all teams became routine. Data collection ended with completion of the team process. This comprised completion of all teams' four hui, implementation of agreed strategies, and individual interviews.

\section{NEGATIVE CASE ANALYSIS}

The purposes of negative case analysis are to draw out knowledge of factors generating particular sets of perceptions and ensure appreciation of diverse

21 Māori language

22 And with a personalized card for each participant 
Table 3.6: Time spent in the field

\begin{tabular}{|c|c|c|c|c|c|c|c|c|}
\hline Dates, 2011 & Activity & Mon & Tues & Wed & Thurs & Fri & Sat & Sun \\
\hline $7-13$ Feb & Recruitment & & & & & & & \\
\hline \multicolumn{9}{|l|}{$14-20$ Feb } \\
\hline \multicolumn{9}{|l|}{$21-27$ Feb } \\
\hline 28 Feb - 6 Mar & Recruitment & & & & & & & \\
\hline $7-13 \mathrm{Mar}$ & Recruitment & & & & & & & \\
\hline $14-20 \mathrm{Mar}$ & Recruitment & & & & & & & \\
\hline $21-27$ Mar & Recruitment & & & & & & & \\
\hline \multicolumn{9}{|l|}{$28 \mathrm{Mar}-3 \mathrm{Apr}$} \\
\hline \multicolumn{9}{|l|}{$4-10 \mathrm{Apr}$} \\
\hline \multicolumn{9}{|l|}{$11-17$ Apr } \\
\hline $18-24$ Apr & & $\mathrm{H}$ & $\mathrm{H}$ & $\mathrm{H}$ & $\mathrm{H}$ & $\mathrm{H}$ & $\mathrm{H}$ & $\mathrm{H}$ \\
\hline 25 Apr - 1 May & & $\mathrm{H}$ & $\mathrm{H}$ & $\mathrm{H}$ & $\mathrm{H}$ & $\mathrm{H}$ & $\mathrm{H}$ & $\mathrm{H}$ \\
\hline 2-8 May & Recruitment & & & & & & & \\
\hline \multicolumn{9}{|l|}{$9-15$ May } \\
\hline $16-22$ May & Recruitment & & & & & & & \\
\hline \multicolumn{9}{|l|}{$23-29$ May } \\
\hline 30 May - 5 June & Data collection & & & & & & & \\
\hline $6-12$ June & $\begin{array}{l}\text { Recruitment and } \\
\text { data collection }\end{array}$ & $\mathrm{H}$ & & & & & & \\
\hline 13 - 19 June & Data collection & & & & & & & \\
\hline 20 - 26 June & Data collection & & & & & & & \\
\hline 27 June - 3 July & Data collection & & & & & & & \\
\hline $4-10$ July & Data collection & & & & & & & \\
\hline 11 - 17 July & Data collection & & & & & & & \\
\hline 18 - 24 July & Data collection & $\mathrm{H}$ & $\mathrm{H}$ & $\mathrm{H}$ & $\mathrm{H}$ & $\mathrm{H}$ & $\mathrm{H}$ & $\mathrm{H}$ \\
\hline 25 - 31 July & & $\mathrm{H}$ & $\mathrm{H}$ & $\mathrm{H}$ & $\mathrm{H}$ & $\mathrm{H}$ & $\mathrm{H}$ & $\mathrm{H}$ \\
\hline 1-7 Aug & Data collection & & & & & & & \\
\hline $8-14$ Aug & Data collection & & & & & & & \\
\hline $15-21$ Aug & Data collection & & & & & & & \\
\hline $22-28$ Aug & Data collection & & & & & & & \\
\hline 29 Aug - 4 Sept & Data collection & & & & & & & \\
\hline $5-11$ Sept & Data collection & & & & & & & \\
\hline $12-18$ Sept & Data collection & & & & & & & \\
\hline $19-25$ Sept & Data collection & & & & & & & \\
\hline \multicolumn{9}{|l|}{26 Sept - 2 Oct } \\
\hline \multicolumn{9}{|l|}{$3-9$ Oct } \\
\hline $10-16$ Oct & Data collection & $\mathrm{H}$ & $\mathrm{H}$ & $\mathrm{H}$ & $\mathrm{H}$ & $\mathrm{H}$ & $\mathrm{H}$ & $\mathrm{H}$ \\
\hline $17-23$ Oct & & $\mathrm{H}$ & $\mathrm{H}$ & $\mathrm{H}$ & $\mathrm{H}$ & $\mathrm{H}$ & $\mathrm{H}$ & $\mathrm{H}$ \\
\hline $24-30$ Oct & & $\mathrm{H}$ & & & & & & \\
\hline $31 \mathrm{Oct}-6 \mathrm{Nov}$ & Data collection & & & & & & & \\
\hline $7-13$ Nov & Data collection & & & & & & & \\
\hline $14-20 \mathrm{Nov}$ & Data collection & & & & & & & \\
\hline $21-27$ Nov & Data collection & & & & & & & \\
\hline 28 Nov - 4 Dec & Data collection & & & & & & & \\
\hline
\end{tabular}

Key: Table 3.6

Time spent in the field

Researcher was overseas

H School closed for holiday 
perceptions. In this study, findings from Team D, a negative case, are presented in detail. Analysis of Team D data contributes to new knowledge about factors that impact on achievement of valued outcomes. Within other teams, variations in valued outcomes experienced (e.g., for Team A students) are explored fully to identify relevant factors.

Considering data from Team D, I knew I must be very careful about my interpretation. Like Fine and Weis (2002), I was worried about finding myself "differentially theorizing and contextualizing voices" (p. 287). Fine and Weis (2002) reflected that they allow "historically smothered" (p. 287) participants to speak for themselves, but in presenting voices of those showing no empathy or understanding of those in "pain and plight, (they) theorize generously, contextualize wildly, rudely interrupting them to frame them" (p. 287). They pondered, "is this an epistemological double standard in need of reform, or is it a form of narrative affirmative action, creating discursive spaces where few have been in the past?" (p. 287). Therefore, I focused on considering various alternative interpretations of negative situations and perceptions, and relied on my supervisors' guidance to help me identify times when my interpretation needed further examination.

\section{MEMBER CHECKING}

In the interests of authenticity and manageability for participants, member checks were conducted. Summaries of data were shared with participants, with the invitation to make any necessary corrections. I summarized data from participants at diverse times: during data collection (e.g., paraphrasing a participant's words to check my understanding), at the close of interviews (i.e., summarizing what has been said), within interpretive FGs, and after analysis (all of which are discussed further below).

Interpretive FGs extended member checks by allowing me to ask participants to check my interpretation of their words, to help me understand their precise meaning. Interpretive FGs enlisted participants' cognitive involvement with data, to enhance the "fairness, relevance and accuracy" (Cohen et al., 2007, p. 70) of representation of participants' thoughts, ideas, and experiences. During later team hui and interviews, I also asked questions about participants' comments or actions in previous events, inviting comment, elaboration, or explanation.

I met with Team B participants after analysis to share findings on two different themes, to check my understanding against their own interpretation. Firstly, I met with Team B parents to share my understanding of participants' experiences within teams, as part of my preparation to present findings on this theme at an international conference. Secondly, I met Team B's parents and teacher to discuss my 
interpretation of interaction within the team. I believed the team was enacting the Māori concept of whanaungatanga ${ }^{23}$, but, as a Pākehā researcher, it was crucial for me to seek the views of the team's Māori participants. Therefore, when we met, I explained I had developed an idea after analyzing data from their team, but wanted to seek their views, as they were the participants; because it was their team, their understanding was paramount. I asked for their comments on a diagram that illustrated my understanding of whanaungatanga, as well as their view of the team interaction. Was it appropriate to characterize it as whanaungatanga in action? This one-hour meeting refined my understanding of whanaungatanga, confirmed my interpretation, and provided further data.

\section{TRIANGULATION}

Triangulation was used to confirm reliability of conclusions by examining data for corroboration and coherence (Hall, 2007). Triangulation to corroborate evidence from various sources included methodological triangulation (Denzin, 1989): diverse data collection methods (e.g., observation, hui transcripts, reflective journal entries, interviews and FGs). Data triangulation (Denzin, 1989) comprised data collection from different sources (parents, students and teachers, across various times and places, and across teams). These processes strengthen proof of both corroboration and coherence: how thoroughly evidence can describe the complete situation. Further triangulation arises from investigation of five cases.

Other conceptualizations of credibility for case studies relate to their ability to meet users' needs. For instance, Gall et al. (2007) contend credibility is demonstrated by:

- Usefulness: enlightening for readers;

- Participatory models: with involvement throughout the study;

- Chain of evidence: providing an audit trail of the research process;

- Reporting style: presentation of findings captures participants' authentic words and experience, thus demonstrating verisimilitude;

- Quasi-statistics: incorporation of numerical results to assess the weight of evidence and support conclusions.

Altheide and Johnson (1994) also emphasize full attention to contextual completeness, including due attention to multiple perspectives and participants' tacit knowledge, and consideration of impacts from researcher positioning.

23 Whanaungatanga is a complex Māori concept that encompasses ideas about principles that should be upheld in relationships (see Section 5.3.2.2.) 


\subsubsection{Transferability}

According to Mertens (2005), transferability may be judged on the "extensive and careful description of the time, place, context, and culture" (p. 256), which allows the reader to assess applicability of findings to other contexts. Although multiple case studies do not claim statistical generalizability, Yin (2009) argues that this approach enhances analytical generalizability of theory generated from findings. These two aspects of transferability as they apply to this study are discussed below.

\section{THICK DESCRIPTION}

Altheide and Johnson (1994) define thick description as contextual completeness. They contend high quality research reports show comprehensive consideration of contextual features in interpretation of data, including attention to multivocality and participants' tacit knowledge (implicit meanings within non-verbal communication, use of humour and nuances). Regarding tacit knowledge, I used two strategies. One was seeking clarification from participants, either by asking questions or listening for contextual clues. My second strategy was drawing on knowledge of Māori and Pasifika students' verbal and non-verbal language, developed through many years of working with them as a high school teacher.

Altheide and Johnson (1994) also argue that verisimilitude (writing which successfully draws the reader into the participants' world) is achieved by drawing on participants' actual words and experience. Therefore, in reporting findings, I have relied heavily on participants' own words (Gall et al., 2007). However, reported findings arise not only from observation, but also reflection (Stake, 2000), including my interpretation of data collected, developed through triangulation and analysis.

\section{MULTIPLE CASES}

Five case studies were conducted simultaneously, somewhat like a series of parallel "experiments" (Yin, 2009). In each team I enacted essentially similar procedures and ways of being. Similarities related to: facilitation techniques and resources; how I interacted with participants; the schedule of data collection activities; questions and sources of evidence. In conducting a multiple case study, the potential for "analytic or theoretical generalization" (Robson, 2011, p. 140) and development of theory from experiences of different cases are strengthened.

Stake (2000) identified an inherent risk for researchers undertaking multiple case study; his concern was "with concentration on the bases for comparison, uniquenesses and complexities will be glossed over" (p. 444). Therefore, to avoid giving insufficient attention to each case (Wolcott, 1992), I undertook deep analysis of each case one by one (Flick, 2006). 


\subsubsection{Dependability}

For constructivist studies, dependability means providing a chain of evidence outlining details of the research process, so its quality and suitability may be assessed. In terms of suitability of the methodology, qualitative research may be assessed by evaluation of: evidence for findings, appropriate choice and application of methods, relevance of results, and researcher reflexivity (Flick, 2006). In terms of research process quality, an audit trail should explicitly present the researcher's aims and worldview, data sources, and procedures.

Samples of materials in key processes provided within this chapter verify authenticity of reported processes and procedures. Various forms of data collected are available for re-analysis by others, including photographs (Flick, 2006), video footage, and audio recordings. These data documented settings, artefacts (such as personal presentations), procedures (such as facilitation techniques), and processes (such as team hui) within the study, each capturing a unique set of relevant aspects. For instance, video footage conveyed non-verbal data (Flick, 2006), and audio recordings captured a range of audio qualities, including tone, volume, silences, and words. Photographs captured summaries of data collected in FGs recorded on a whiteboard, when participants agreed the record was true and complete, and audiorecordings provide verification. Thus, collection of data avoided reliance on "ephemeral" observations (Flick, 2006, p. 240) and repeated viewings supported researcher reflexivity.

\subsubsection{Confirmability}

As for dependability, an audit process assesses confirmability. Because "data, interpretations and outcomes are rooted in contexts and persons apart from the researcher" (Mertens, 2005, p. 15), confirmability is a key principle for constructivist studies. Careful linking of data to sources, and detailed explanations of the evidential basis for interpretations within presentation of findings will allow readers to assess confirmability for this study.

Although there are risks associated with having one researcher - confirming the importance of triangulation strategies - Janesick (2003) argues this approach is in line with qualitative research methodology. Discussing the researcher as a research instrument, she compares the work to that of a choreographer, involved in a particular contextualized shared experience with participants. In accordance with her recommendations, I endeavoured to declare my biases and assumptions to participants, including my desire to investigate whether application of the notion of FoK has value with the NZ high school context. My research journal provides an audit trail of the research process, decision-making within it, and reflections on the 
influence of my words and actions on the process and outcomes. Reviewing data and revisiting coding decisions, as well as drawing on advice of my supervisors and peers, also assisted my efforts as a sole researcher to be consistent.

To strengthen confirmability, when undertaking data analysis, I worked to suspend my own bias and expectations in order to avoid hearing what I expected to hear from participants, and increase the likelihood of understanding real meaning. My supervisors and peer mentors asked challenging questions, forcing me to examine my assumptions and be more critical about my own thinking. Multiple viewings/readings of data were also helpful, as was reliance on participants' own words as much as possible in summarizing their ideas (Hycner, 1985). As Eichelberger (1989) noted, constructivist researchers "are constructing the 'reality' on the basis of interpretations of data with the help of the participants who provided the data in the study" (p. 9). Use of interpretive FGs (Dodson \& Schmalzbauer, 2010) further supported accurate representation of participants' perceptions.

\section{CODING CHECK}

This aspect of confirmability is demonstrated through explanations of category development and coding decisions, described in Section 3.8.5. Coding checks also encompass assessing reliability in coding, further enhanced by discussions with my supervisors and peer briefings.

\subsubsection{Authenticity}

Whether a research report is authentic relates firstly to how well it presents a "balanced view of all perspectives, values, and beliefs (Lincoln \& Guba, cited by Mertens, 2005, p. 257). Writing for an audience of ethnographers, Eisenhart (2001) outlines the complexity of this challenge for researchers (like me) working with groups to investigate interventions, and bring about change:

As soon as multiple and competing voices must be represented in a group, the situation becomes more complicated. How should divergent voices be handled when decisions had to be made? Whose needs and desires should have most weight when resources are limited? What should one do when needs and desired are contradictory? Is it appropriate for one to intervene without consensus, and on what grounds? Deep appreciation of variety and multiple perspectives within groups (not to mention uncertainty about the appropriateness of a researcher's commitments) makes decisions about change or intervention even more difficult than they have been. (p. 19)

To this end, fairness is judged by presentation of conflicts and value differences (Mertens, 2005). Less than full reporting would threaten generation of relevant theory; therefore, in presenting case findings, I endeavoured to highlight complexities, tensions and contradictions. Ultimately, readers will judge this 
element. The second element of authenticity, catalytic authenticity, is demonstrated by stimulation to action resulting from the study (Mertens, 2005), evidenced by teachers' statements of intent and actual changes in practice.

\subsection{Conclusion}

In response to Eisenhart's (2001) challenge, this qualitative study draws from constructivist and emancipatory perspectives, and utilizes a blend of methodological elements appropriate to the research purpose and questions. An approach featuring team-based collaboration (TBC) was designed, in order to explore student, parent, and teacher participants' perspectives related to the application of a FoK approach in a NZ high school.

To provide clarity to the reader, findings related to the actual experience of fieldwork as the TBC approach was conducted are presented in Section 9.3. These findings highlight supports and challenges experienced, and include analysis and discussion of the researcher role. ${ }^{24}$

\footnotetext{
${ }^{24}$ Because of references to specific team participants and processes, it was judged that these findings would be more understandable to the reader when presented after findings related to each team. Therefore, these general findings related to the fieldwork experience are presented within Chapter 9: Cross-team Findings.
} 


\section{Chapter 4: Team A Findings}

\subsection{Introduction to Team A}

Team A's teacher was Lizzie, a senior manager and Science teacher at KHS. Lizzie had immigrated to New Zealand as an adult, from the United Kingdom. She had taught at KHS for nine years of her twenty-year career. Lizzie described herself as having some words and phrases in Te Reo Māori.

The student participants were Thor and Sonny Bill, both in Lizzie's Year 9 Science class. Thor identified himself as Māori/Cook Island. His first language was English, and he spoke some Māori. When the study began, he was 13 years and two months of age. The statement he said best described him in his Science class was "okay." He identified his relationship with Lizzie as "sometimes difficult," while Lizzie perceived it as "mostly good;" however, she was concerned that he was truanting class, and was not actively engaged when present. Sonny Bill identified himself as Māori/European. When the study began, he was 13 years and eight months old. His native tongue was English, and he knew some Māori words and phrases. The statement he said best described him in the Science class was "mostly I enjoy it and it's going pretty well." He categorized his relationship with Lizzie as "mostly good," and Lizzie concurred.

The parent participants were Ses and Carol. Ses, Sonny Bill's stepfather, was a Pākehā man in his forties who worked in a professional role. Carol, Thor's mother, a Māori woman, worked fulltime in a skilled job and was undertaking tertiary study. Carol withdrew before Hui 3. Lizzie had no previous interaction with Carol or Ses.

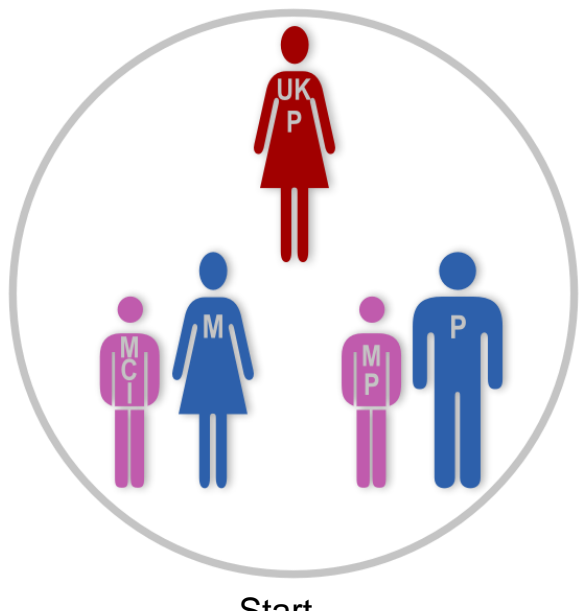

Start

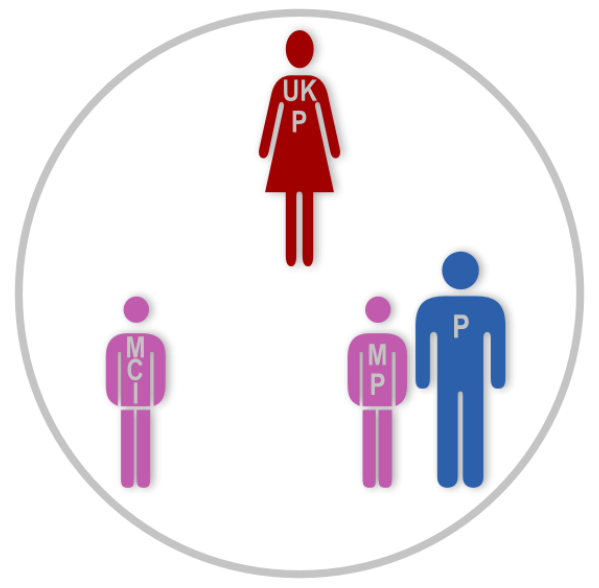

End

Figure 4.1: Team A participants at start and end of study

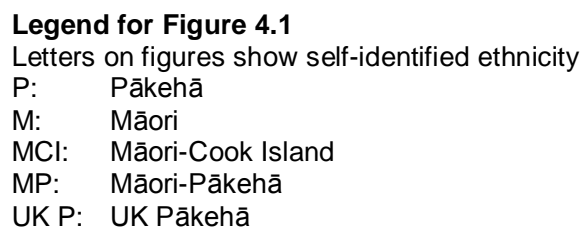




\subsection{Synopsis of findings}

All Team A participants hoped the students' educational achievement would improve when Lizzie learnt about their FoK. Lizzie had well-developed ideas of how knowledge of students' FoK could help her; she hoped to gain a better understanding of them as people, to develop closer relationships with their families, and to develop more relevant learning experiences, aligned with and integrating students' FoK. Ses saw the students' time at secondary school as high stakes, because being left behind could threaten their career preparation. Thor hoped for "better work, more fun work" (reflection), and hinted at his desire for academic identity, saying he hoped the project would help him "go to university, then I could talk about this project" (reflection).

Team events were characterized by inclusive discussion of a range of options. Chosen strategies emerged clearly from discussion, and were agreeable to all. In Hui 2 the team agreed to all share personal profiles at a special TM (TM1). In Hui 3, Lizzie reported she learnt about Sonny Bill's and Thor's lives and values and was developing closer relationships with them. However, she felt she still did not know much about their skills and strengths, and wanted to develop ideas about applying their FoK to classroom learning. With this in mind, Lizzie asked if the boys would collaborate with her to plan learning topics for the current unit of work. They were thrilled to do this, but implementation was less successful, because the unit went on too long.

Despite ongoing absence and lateness issues, positive valued outcomes were evident. Thor was absent from both TMs (TM1 and TM2) to implement agreed strategies. Thor's mother was absent from TM1, and subsequently withdrew. Additional TMs scheduled to accommodate Thor (TM1b and TM2b) went ahead successfully, and thus agreed strategies were implemented. Thor was regularly late to team hui. Nevertheless, gains from participation for Thor were emphatically positive (see Section 4.3.4).

All members reported developing deeper understanding of each other. Their interaction enhanced teacher-student and teacher-parent relationships, by helping them see each other as people. At the beginning of the study Thor had been marginalized in Science, and at the end improvements in attendance, engagement, and perseverance were evident. Sonny Bill's positive outcomes, including greater enjoyment of Science and a closer relationship with Lizzie, were comparatively modest. New pedagogical practices for Lizzie included co-constructing unit plans with the class and learning activities drawing on students' diverse FoK. 


\subsection{Evidence by theme}

\subsubsection{Preferred approaches for teacher to learn about students' FoK}

Team A's agreement from Hui 2 stated

Everyone in our team will make a personal profile to share with each other. (Team A agreement 22-06-11.pdf)

TM1, to implement this strategy, was scheduled for a Thursday evening three weeks hence.

In Hui 3, Lizzie suggested a further strategy because she wanted to learn how to apply students' FoK to teaching practice. All members were enthusiastic, and it was agreed:

Thor and Sonny Bill will share their ideas with Lizzie about topics that could be part of the "Diego Run" Science unit. These ideas could be things to learn about, or ideas for activities, and could be things that their family has expertise in. (Team A agreement Hui 3.pdf)

The unit planning consultation (TM2) was scheduled the following week.

\subsubsection{Enablers that supported valued outcomes in Team A}

\subsubsection{Inclusive team environment}

In her interview, Lizzie stated:

It's the human interaction where you get the change happening. So yes, we provided a structure for that human interaction to take place but the change came from that interaction between the people who were in the team. (lines 424-426)

In Team A, the nature of interaction in TBC supported valued outcomes. In FGs and interviews participants stated they were respected and listened to, confirming my observations. Various actions by members contributed to a positive, inclusive environment. Examples are listed in Table 4.1.

Positive interactions showed members could trust each other, leading to relaxed communication and development of rapport. Lizzie saw this as an essential foundation to collaboration. Attention to relationship development underpinned Lizzie's actions, illustrated in Table 4.1. Lizzie stated "I'm interested in people, I'm interested in kids, I like them, and I'm happy to know more about them (interview, lines 474-475). I noted I never heard her make any negative remarks about students. She had participated in E Tipu E Rea, TPL that focused on qualities of relationships valued by Māori students. Lizzie stated that she had experienced outsider status as a immigrant, and was conscious of how exclusion or inclusion occur. 
Table 4.1: Examples of positive inclusive actions in Team A

\begin{tabular}{|c|c|c|c|}
\hline $\begin{array}{l}\text { Positive inclusive } \\
\text { actions }\end{array}$ & Who? & When? & What? \\
\hline \multirow[t]{3}{*}{$\begin{array}{l}\text { Acknowledging } \\
\text { student achievement }\end{array}$} & Lizzie & Hui 2 & $\begin{array}{l}\text { "I was hearing about your inventions today... } \\
\text { sounds like some good inventions there though." }\end{array}$ \\
\hline & Ses & TM1 & $\begin{array}{l}\text { "He'd only learnt the song the night before! He } \\
\text { didn't even know how to play ukulele!" }\end{array}$ \\
\hline & & & "You're a good big brother." \\
\hline \multirow{4}{*}{$\begin{array}{l}\text { Positive verbal } \\
\text { response to } \\
\text { personal information }\end{array}$} & Lizzie & Hui 2 & "Oh awesome, what a big family!" \\
\hline & & Hui 3 & "We saw that statue" \\
\hline & $\begin{array}{l}\text { Sonny } \\
\text { Bill }\end{array}$ & & "On Geordie Shore" \\
\hline & Lizzie & TM1b & "That's really good to hear Thor." \\
\hline \multirow{3}{*}{$\begin{array}{l}\text { Questions which } \\
\text { show interest in } \\
\text { personal information } \\
\text { shared }\end{array}$} & Lizzie & TM1 & "Do you play any other instruments?" \\
\hline & Ses & & "And why do you want to join the army?" \\
\hline & Linda & & $\begin{array}{l}\text { "Can I ask what else did you have to do at the } \\
\text { wedding?" }\end{array}$ \\
\hline \multirow[t]{2}{*}{$\begin{array}{l}\text { Positive non-verbal } \\
\text { response to } \\
\text { personal information }\end{array}$} & All & TM1 & $\begin{array}{l}\text { Clapping at beginning and end of presentation of } \\
\text { personal profile }\end{array}$ \\
\hline & & & Attentive listening \\
\hline \multirow{2}{*}{$\begin{array}{l}\text { Welcoming } \\
\text { comment to late } \\
\text { arrivals }\end{array}$} & Linda & Hui 2 & "How nice to see you!" \\
\hline & Lizzie & Hui 3 & "Here he is! You made it, well done!" \\
\hline $\begin{array}{l}\text { Bringing latecomers } \\
\text { up to speed }\end{array}$ & Linda & Hui 4 & $\begin{array}{l}\text { "We were just starting to have a talk about what it } \\
\text { was like to be in a team" }\end{array}$ \\
\hline $\begin{array}{l}\text { Positive response to } \\
\text { strategy suggestion } \\
\text { despite another } \\
\text { preference }\end{array}$ & Lizzie & Hui 2 & $\begin{array}{l}\text { "I like the idea of the journal too; also I'm aware } \\
\text { that I don't want Sonny Bill to feel under scrutiny. } \\
\text { Another idea I liked was the idea of bringing } \\
\text { things to school" }\end{array}$ \\
\hline \multirow{3}{*}{$\begin{array}{l}\text { Asking students for } \\
\text { their opinion /ideas }\end{array}$} & Lizzie & Hui 2 & "What do you think about that Thor?" \\
\hline & Linda & & "Who'd like to start?" (looking at Sonny Bill) \\
\hline & Ses & Hui 3 & "Do you want to be involved in the planning?" \\
\hline $\begin{array}{l}\text { Appreciation of } \\
\text { participation }\end{array}$ & Lizzie & Hui 4 & $\begin{array}{l}\text { "What helped me in terms of the team working was } \\
\text { everyone's commitment, you know you guys never } \\
\text { missed a meeting, you always turned up, and you } \\
\text { know were all committed to this and followed } \\
\text { through, and I think that's been really cool" }\end{array}$ \\
\hline
\end{tabular}

Lizzie also commented about my input as researcher: she considered my participant observer role in teams, "helped bind it all together" (interview, line 574), and relationship development included me too. Table 5.2 shows I played a role in establishing an inclusive team environment. A further example was my organization of rescheduled TMs. The success of these evidences participants' flexibility to accommodate individuals, which was crucial to Thor's achievement of valued outcomes. 
Thor felt the small team size encouraged commitment and accountability:

'Cos smaller teams are harder teams than big I think. Bigger teams are not all that confident cause in little teams you would have to think about your own ideas and in big teams they have different people talking and you were just sitting there like, yes, all I need to do really. (interview, lines 538-541)

Therefore, in his view, because each member was highly visible and actively involved within the small team, this feature promoted inclusivity. Nevertheless, Thor stated he did not express all his ideas/ thoughts.

\subsubsection{Strategies were valued by participants}

Both strategies agreed had full support of all members throughout discussion and negotiation. During Hui 2 several strategies were suggested, but Ses's idea of a personal profile was discussed enthusiastically by all; consensus was readily apparent. In Hui 3, Lizzie's request that the students make suggestions for unit planning was also received enthusiastically.

Features of the personal profile activity members liked were open-endedness, manageability, and opportunity for everyone to learn about each other. Thor stated this was his favorite part of what the team did. However, because he was absent from TM1, Thor actually shared his personal profile with Lizzie and Sonny Bill at TM1b. (See Section 4.3.3.2 for further discussion of issues arising from Thor's lateness and absence.)

All members reported it was easy to decide what information to share, and individuals chose different ways to present their profiles. It was agreed others could ask questions during presentations, but mostly this occurred afterwards. Lizzie enjoyed the reflection involved in developing her profile; she prepared a poster depicting her life as a journey south, illustrated with personal photos and maps. Ses also made a poster, using a rugby jersey as a backdrop for photos and symbols. He believed the task was worthwhile and liked the inherent autonomy; he could choose to share "not necessarily deep, dark secrets you know but just things that make you tick and what you enjoy you know, that sort of stuff" (Hui 4, lines 134-136). Sonny Bill also brought a poster, consisting of five pictures, which related to family, interests and goals.

In contrast, in TM1b, Thor made no use of artefacts. He began his presentation with amusing stories about how as a baby he was so fat he broke his cot, and could not fit in a jumper. After the stories, I remarked "How interesting!" and then suggested Lizzie and I swap places, so she and Thor could make eye contact (previously I was opposite him, and Lizzie sat to the side). At this point, when Lizzie moved to sit across the table from him, Thor changed roles from storyteller to interviewee, adopting a 
pattern of waiting for his teacher's questions. It is unclear why the dynamic shifted, but I wondered whether positioning teacher and student across a table from each other evoked their traditional roles. Alternatively, I wondered whether Thor interpreted lack of verbal feedback about his stories as lack of interest. Thus, from that point, Lizzie sat across the table from Thor, leaning forward, maintaining eye contact, guiding his contributions with questions. Sonny Bill and I also asked questions. Thor showed desire to share information by elaborating on answers. For instance, when Lizzie asked if he saw his older brother much, he stated:

Yeah, he comes home all the time, but he always goes back to Dusky Bay. He comes home for the money, and mum was like no. (TM2, 10m.09s-10m.21s)

When Thor stated his rugby league position, I asked him for clarification:

Linda: I don't know much about league, what does that mean?

Thor: You're like, a front forward, you're meant to like, tackle and everything. Like you're meant to do all the hard work, until the backs peuhh! ${ }^{25}$ Then they're like (unclear). It's like sad, 'cos none of the forwards get any tries, 'cos they have to pass it out to the backs. (TM2, 6m.54s-7m.21s)

However, most questions were closed, and failed to open up conversation, even though Lizzie provided space afterwards. An example is provided for illustration.

Lizzie: So your family are from the Cook Islands?

Thor: Just my dad

Lizzie: And what about your mum?

Thor: She's full Māori

Lizzie: Where's she from?

Thor: Paraparaumu. (TM2, 5m.14s-5m.29s)

Later Lizzie and I realized our actions unintentionally constrained Thor's presentation (see Section 4.3.3.1).

Learning about each other through personal profiles was highly valued by all. Learning about each other appeared to be more highly valued than the opportunity to share about oneself, as suggested by the proportion of participants' comments relating to these two aspects (see Section 4.3.4).

Both students were thrilled to contribute to planning the "Diego Run" Science unit. They thought it was "cool to see what we can do with the teacher" (Sonny Bill, interview, line 26) and "actually (get) to decide what we want to do" (line 93). Later both stated they would happily participate in co-construction of future unit plans. As for

${ }^{25}$ to show the idea of fast running 
students in Teams D and E, Thor and Sonny Bill's class was engaged in an IS program. Themes from the novel Diego Run, which the students had read in English, provided the focus for the current unit of learning. As soon as the collaborative planning session was agreed, the boys began to articulate ideas.

I facilitated the collaborative planning session (TM2b) in a way I hoped would stimulate ideas: firstly Sonny Bill and Thor summarized the novel's plot, then recalled topics already studied, finally brainstorming learning ideas. They suggested topics they wanted to learn about (e.g., what different drugs do to you and how they make you addicted), as well as topics related to their FoK and personal goals (e.g., how to look after your body and avoid injury when you play sport). Therefore, suggested topics related to their interests and ideas about worthwhile knowledge.

A range of factors appeared to affect students' confidence to contribute in TM2b. Thor's family's FoK gave him confidence. He stated:

I know we've got good ideas, it's like, yeah, that's... only 'cos my mum used to be a nurse and then she didn't want to be that and then she became a radio station person, then now she's doing midwifing (sic). (interview, lines 257-259)

Observing TM2b, I reflected that Thor's and Sonny Bill's knowledge of the novel and the absence of technical language to identify the unit made the task manageable. The biggest challenge for the students was not having a sense of parameters for a valid suggestion (i.e., relevance to Science, and any other parameters Lizzie held). However, Lizzie's openness to students' ideas was evident from her positive, encouraging responses. For example, often suggestions were expressed as questions, and Lizzie's tone confirmed their validity; at times she elaborated suggestions to clarify their relevance.

However, at times I was unsure whether Lizzie's elaboration maintained the students' intended focus. For instance, in a discussion regarding learning about effects of drugs on bodies, she suggested an experiment to identify effects of coffee. At the time, coffee was a very fashionable drink, and sourcing high-quality coffee was a popular adult conversation topic. At team events, Ses drank coffee, but Sonny Bill drank soda. Also, as mentioned in Hui 3, Sonny Bill was not allowed to drink coffee, and Thor drank neither tea nor coffee due to his religious beliefs. Therefore, Lizzie's suggestion of investigating coffee's effects lacked direct relevance to Sonny Bill and Thor, although it may have been relevant to other family members. Possibly Lizzie's suggestion arose because coffee is one of few drugs with which students could physically experiment.

Not all ideas from TM2b were implemented (see Section 4.3.3.3). 


\subsubsection{Barriers to valued outcomes in Team A}

4.3.3.1 Learning about students' strengths and skills was challenging

Although personal presentations generated valued outcomes (see Section 4.3.4), in Hui 3 Lizzie reported she still did not have a clear sense of Thor's and Sonny Bill's strengths and skills.

Sonny Bill's personal presentation and discussion afterwards was almost eleven minutes long. He talked about his extended family, Ses and his mother's recent wedding and his role in it, music, and his ambition to join the army. Ses interrupted to proudly add details, give praise, and occasionally prompt him. Other information about Sonny Bill emerged in discussion of Lizzie's presentation. For instance, when Lizzie stated her family does not have a television, Sonny Bill commented they have televisions in most rooms. In his seven-minute presentation, Ses talked about his involvement playing rugby and cricket, sports awards won, his favourite beer, DIY, his wedding, and family. Some comments clarified his influence on Sonny Bill's values and hobbies, such as trying to promote Sonny Bill's interest in DIY. Thor's talk included babyhood stories and descriptions of immediate family members.

Thor, Lizzie and I agreed Thor's presentation could have been more successful. The presentation was done without any visual aids and lacked fluency, and Lizzie and I sought to prompt him with questions. However, we later reflected our questions guided his content, and would not have helped him to present the information intended. In his interview Thor stated he realized later he forgot some things he intended to say. He said "If I got to do it twice it would have been way better 'cos I knew what I would be saying and I would have a long time" (lines 47-48). In response to my question about what he would add, he said "What I do in life, like I like sport, playing sports, and all my history and all that, and what I want to be when I grow up" (lines 53-54). Although Thor didn't identify prompting by Lizzie and I as a contributing factor, I believe it played a significant role in distracting him.

Lizzie stated the students were modest; although they were willing to talk about themselves, they didn't tend to do so in terms of things they were good at or in which they had expertise. Consequently, Lizzie found "as an individual trying to draw out from the kids what they think their skills and strengths are, it's pretty difficult" (interview, lines 98-99).

An additional element that contributed to Lizzie's difficulty may have been lack of familiarity with observing data to identify FoK. She was confident her intention to learn about students' FoK was sufficient to "look with a different pair of glasses" (interview, line 469). But I noticed some themes discussed by students provided information that 
could have been further teased out to clarify FoK, such as discussion of music and television. Lizzie reviewed some video footage of personal profiles at TFGs, and found collaborative reflection and review of footage valuable "because I think you have a perception of what you thought you saw and heard at the time and your memory is a very strange thing" (interview, lines 480-482). Therefore, I argue further viewings may have deepened Lizzie's understanding of students' FoK.

Lizzie reflected in her interview that she gained clarity about what she wanted to learn about the students, suggesting her future approach to learning about students' FoK would be more tightly focused. She considered parents were a good source of information about students' FoK, as they were more likely than students themselves to be willing and able to describe students' FoK. Lizzie saw this as a good reason to involve parents as partners to support teachers learning about students' FoK. Thor also commented if a parent from his family had participated, "there would be more ideas in there... way more ideas... ideas of the family, our family" (interview, lines 438-444).

Although Ses mentioned Sonny Bill several times in his presentation, Lizzie did not see his presentation as contributing to her understanding of Sonny Bill's FoK. She considered "the way we set it up, we didn't actually articulate that that was what we wanted to know" (interview, line 122-123).

\subsubsection{Problems arising from attendance issues}

For 6 team events, at least one participant from Thor's family was either absent or late, as shown in Table 4.2.

Although scheduled events were preceded by a 15 minute 'buffer' period, to promote full attendance and timeliness, this did not work for Team A, and findings indicated some implications arising.

Each time Team A met, different factors affected Thor and /or his mother's attendance. No reason was asked for or given for Carol's absence from Hui 1. When Thor and Carol arrived 15 minutes late for Hui 2, Carol stated she had been working, and at Thor's insistence stopped work early to attend. Three weeks later, Thor and Carol did not attend TM1. Neither Lizzie nor I knew the reason or meaning of this absence. On the occasion of Hui 3, I had arranged to transport Thor, but when I arrived at the agreed place and time he was not there. It transpired that due to a misunderstanding, Thor had bussed home, and his father brought him by car to Hui 3. Thus, he arrived eleven minutes late. On another occasion, shortly before TM2 Thor's father removed him from school for a shopping trip. Finally, Thor was 28 minutes late for Hui 4. No reason was asked for or given. 
Table 4.2: Attendance, absence and lateness in Team A

\begin{tabular}{|c|c|c|c|c|}
\hline Research event & Present & Absent & Late & $\begin{array}{l}\text { Presence not } \\
\text { requested }\end{array}$ \\
\hline Hui 1 & $\begin{array}{l}\text { Lizzie } \\
\text { Ses } \\
\text { Sonny Bill } \\
\text { Thor }\end{array}$ & Carol & & \\
\hline Hui 2 & $\begin{array}{l}\text { Carol } \\
\text { Lizzie } \\
\text { Ses } \\
\text { Sonny Bill } \\
\text { Thor }\end{array}$ & & $\begin{array}{l}\text { Carol } \\
\text { Thor }\end{array}$ & \\
\hline TM1 & $\begin{array}{l}\text { Lizzie } \\
\text { Ses } \\
\text { Sonny Bill }\end{array}$ & $\begin{array}{l}\text { Carol } \\
\text { Thor }\end{array}$ & & \\
\hline TM1a & $\begin{array}{l}\text { Lizzie } \\
\text { Sonny Bill } \\
\text { Thor }\end{array}$ & & & Ses \\
\hline Hui 3 & $\begin{array}{l}\text { Lizzie } \\
\text { Ses } \\
\text { Sonny Bill } \\
\text { Thor }\end{array}$ & & Thor & \\
\hline TM2 & $\begin{array}{l}\text { Lizzie } \\
\text { Sonny Bill }\end{array}$ & Thor & & Ses \\
\hline TM2b & $\begin{array}{l}\text { Lizzie } \\
\text { Sonny Bill } \\
\text { Thor }\end{array}$ & & & Ses \\
\hline Hui 4 & $\begin{array}{l}\text { Lizzie } \\
\text { Ses } \\
\text { Sonny Bill }\end{array}$ & & Thor & \\
\hline
\end{tabular}

On each occasion when participants arrived late, both Lizzie and I welcomed them. I reiterated what had occurred, while other members waited quietly. Therefore, the patience of others supported inclusivity. I noted in my reflection other team members were very accepting, but it became apparent Ses found it "disruptive" (interview, line 78). When Thor missed TM1 and TM2, these were rescheduled to enable his participation. However, when Carol did not attend Hui 1, I did not offer another opportunity because Team A's Hui 2 took place the following day, and I judged it unreasonable for other team members to reschedule at such late notice.

Team members had different perceptions of absence and/or lateness of members, and different experiences and views regarding impacts. When participants had not arrived, I found this somewhat preoccupying. As several Team A events were held in the evening at school, I needed to keep the building secure. Therefore, I left the room intermittently to check whether anyone else had arrived. Ses articulated frustration about other members' lateness several times, and interpreted it as lack of commitment:

I found it a little bit disruptive. I found Thor coming in late and stuff and handed that back over stuff a bit frustrating, and that's no fault of yours, but it's just people's commitment that I find a little bit annoying. But I still found that the team worked well with Sonny Bill, myself and the teacher, so that was quite cool. (interview, lines 78-82) 
However, Lizzie seemed not to notice all Thor's occurrences of lateness. In a TFG she commented "he was late wasn't he for the last one that we had, so he wasn't particularly reliable" (TFG3, lines 339-341). Considering whether Carol's withdrawal affected the team, Lizzie stated:

It didn't faze me, that's just what happens when you work with people. People have different reasons for being there or not being there, and that's fine, you just work around it I think. I mean, for me, the fact that Thor still managed to have a positive experience, that's what's important. (interview, lines 698-701)

Sonny Bill made no comment about lateness. However, Thor perceived himself as a committed member of Team A. He stated "I think our team was the strongest... being together and sticking together and doing more ideas and bumming the other ones out" (interview, lines 504-514). Thor reported his independent participation (without an accompanying parent) was:

... actually teaching you more. If you're getting the job you won't need your parents there. If you're in the interview, you can't have your parents there by your side and saying stuff, like saying what you had to say, and giving them ideas about your own self. You will need to do it by yourself when you grow older. (interview, lines 458-461)

These comments suggested Thor took responsibility for getting himself to research events. At least twice Thor's absence/lateness was beyond his control, but ultimately he always fulfilled his commitments as a participant. Therefore, different participants' ideas and feelings indicated the contested nature of commitment, highlighting potentially conflicting responsibilities of researchers. Tensions exist between avoidance of imposing one's own definitions and judgement on participants, and respecting participants' time, which had an added complexity within the TBC approach.

Over time I gained an insight into Ses's perspective and needs, highlighting considerations for researchers conducting medium term studies such as this. I got the impression he was impatient of wasted time and liked time to be used productively. His life was very busy at the time, as a fulltime worker, father, and newly married man who was also trying to sell his house and buy a new one. He considered "it's quite a productive size group" (interview, line 144), because most time was spent actively contributing, not simply listening to others. However, Ses was the one participant who stated he "struggled to see my value" (PFG, 11-07-11) in some aspects of the study, and the only one who stated he found the research process "drawn out" (interview, line 319), suggesting the timeframe extended beyond his own perceptions of productivity. Nevertheless, Ses considered it 
important "you commit to it to the end... you're letting the team down if you don't turn up" (Hui 4, lines 628-630), and his reliability demonstrated his belief in action.

Although Lizzie stated she was unfazed by Thor's lateness, she judged there were negative impacts on benefits arising. In Hui 4, Lizzie told her team:

What helped me in terms of the team working was everyone's commitment, you know you guys never missed a meeting, you always turned up, and you know were all committed to this and followed through, and I think that's been really cool. (lines 616-619)

Thus, Lizzie validated all members' participation, mirroring earlier inclusive, encouraging actions (see Section 4.3.2.1). However, in TFG3, she stated:

I think one of the limits in my wee team was the fact that we couldn't pin down Thor's mum and also Thor, you know he came along but... he was late wasn't he for the last one that we had so he wasn't particularly reliable... so they were a limitation really because we didn't get to be able to share and develop in the same way that I did with Sonny Bill and Ses. (lines 338-343)

Nevertheless, strong findings of valued outcomes for Thor suggest Lizzie's perceptions of her improving relationship with, and knowledge of, Thor either were different from his, or not as significant as his experience of being in the team. (See Section 4.3.4 for descriptions of valued outcomes achieved.)

\subsubsection{The co-constructed unit plan went on too long}

Although Lizzie and the students were excited to collaborate for unit planning, implementation was not as successful. When the collaborative planning occurred, the unit was already well underway, and Lizzie later realized the class had had enough of the "Diego Run" focus. Sonny Bill reported this was "the worst bit... that was the only bad thing wrong" (interview, lines 111-115). Lizzie "felt it too, but it was reflected by the fact that you could see, oh the glaze (in Sonny Bill's eyes), and that was a good indicator to me that oh yeah, we're well over this now" (interview, lines 320-322). Consequently, Lizzie concluded the unit and some of the boys' suggestions were not taken up. Although the class studied effects of different drugs on the human body, they did not learn about looking after themselves to avoid sports injuries, which related to Thor's FoK.

As Ses stated, it seemed the timing of collaborative planning was too late. "They're all over "Diego" by then, they had enough of it and it was just going through the motions. So, learning from that, then you'd get them involved earlier on" (interview, lines 578-579). Sonny Bill agreed, stating: 
We have good ideas but we're over the whole topic. It would have been good near the middle or start of the topic, but right at the end, we've basically finished it and we even went more into it. (Hui 4, lines 251-253)

Thus, collaborative planning and implementation lost some potential impact because it felt like "adding more on the top" (Lizzie, Hui 4, line 255).

Lizzie believed the main benefit came from valuing the students' ideas. She stated, "their input was validated and was integrated in what we did in the classroom, and that was good for them. Whether it was hugely successful or not I just don't think really matters" (interview, lines 625-627). Both boys reported Science became "more funner" (Thor, interview, line 83), suggesting that, although the unit was not implemented as intended, what had occurred had been worthwhile.

\subsubsection{Valued outcomes achieved}

\subsubsection{Seeing each other as people}

Sharing personal profiles led to team members' understanding of each other developing greater depth. Both students started to see Lizzie as a person, not just a teacher. Thor said:

It's how... she came from Scotland and came down to New Zealand and started rock climbing and all that, rock climbing and new things that I didn't know. I thought that she was just an ordinary teacher. (interview, lines 32-34)

Lizzie considered she learnt:

... how much community life figured in Thor's experience... the different aspects that he plays within the community... with Sonny Bill I suppose just that whole sense of the importance of his family and how he really values the richness of his family experience. (interview, lines 48-56)

She felt her new knowledge helped break down traditional barriers between students and teachers. She explained:

Traditionally the teacher tried to maintain the distance didn't they, a professional, you know, you'd have to have a professional relationship but they also tried to maintain a personal distance from the students and the parents, whereas I think a project like this and what we did as a team you're actually breaking that down and saying well this is who I am as a person and you know it makes you more human you know. (Hui 4, lines 146-150)

Therefore, Lizzie believed schools, concerned with "a particular sort of business" (interview, line 71), are "artificial communities" (line 70), and rules governing teacher-student interactions prevent dealing with each other as people. She commented that although she knew intuitively "every individual is really complex and rich and wonderful" (interview, line 27-28), limited class time and how she and students present themselves in classroom interactions hide that complexity. 
Ses and Thor discussed their perceptions of what Lizzie had learnt, and why it was important. Ses felt Lizzie learned about their family beliefs, thus clarifying the role Sonny Bill's parents want in his education. He hoped this would lead to an active partnership between them and Lizzie. In contrast, Thor believed Lizzie's knowledge of him would affect how she perceived and treated him:

'Cos if you come to a school and you don't know them, then I think they'll tell you off, but if you know the teacher really well, they'll be like, "Oh yeah, you're a good boy," and you feel like treat you the same with other kids, like older kids that they know. (interview, lines 88-91)

\subsubsection{Enhanced relationships}

Teacher-student and teacher-parent relationships improved for members. Sonny Bill reported "If, just say, I walked past Miss, I'd say hello now. I probably wouldn't do that again to a different teacher" (interview, lines 70-71). He and Ses agreed that Lizzie knowing and caring about the boys generated greater loyalty and respect from them back to her.

For Thor, the improvement in relationship with Lizzie was dramatic. In his interview, he reported his relationship with Lizzie was "super super great" (interview, line 408), whereas previously he described it as "sometimes difficult." One manifestation of their closeness was his sharing of private things with her. For instance, when Lizzie held a baby photo competition (to support learning about genetics), Thor brought in treasured photos, but showed her alone. He also commented Lizzie disciplines him when "I've been naughty, I'm not listening to her, like if I'm playing with my mates and all that in class and just mucking around, and that's when I forget to listen to her" (interview, lines 108-110). Clearly he perceived discipline resulting from behavioral aberrations as not incongruent with the mutual understanding between himself and Lizzie that basically he was a good boy. Also, dramatic changes in Thor's attendance and engagement in class occurred (see Section 4.3.4.3). Lizzie believed Thor's increased engagement was caused by their deeper relationship and knowledge of each other.

Ses was impressed by Lizzie's openness and commitment to get to know the students and improve their learning. Although he noted the relationship was untested by challenges, he stated he now trusted her as a professional:

I'd feel comfortable enough to challenge her if I didn't accept something... yeah, and she'd really validate why she gave those comments, and l'd trust what she's saying. Sometimes you wonder if the teachers actually know the students... and are they just writing down a preamble that they've already got. (interview, lines 392-418) 
He now anticipated meaningful discussions with Lizzie at parent-teacher interviews, because they had "broken the ice and introduced and got to know each other" (Hui 4, line 124). He hoped to work together with Lizzie to support Sonny Bill's learning.

\subsubsection{Improved effective learning behaviors}

Findings related to the two boys as learners in Science were quite different; Thor, who was quite marginalized in class when the study began, experienced dramatic improvement, whereas for Sonny Bill, already well-integrated into the class, improvements were more modest.

Implementation of agreed strategies marked a new sense of agency for Thor as a learner. When the study began, Thor's attendance in Science was irregular, and when present he did not always engage with Lizzie or other students. Reflecting back, Lizzie said "Thor was starting to truant now and again and that's all disappeared so he's coming, he's always in class all the time now" (TFG3, lines 262-263). Thor's involvement in the study generated dramatic improvements. In his interview, his face was radiant and full of joy as he related changes in his school life. Prior to the study, the statement he said best described him in Science class was "okay," but at the end, he was so happy he constructed a new statement beyond the Likert scale provided, implying the top statement in the Likert scale provided ("I really enjoy it and it's going really well") was inadequate:

I would put the top one, the first one, but I will be "I really enjoy it and I'm doing, oh it's going very, very well, 'cos like going super well. (interview, lines 389-390)

Elaborating, he explained his learning in Science was:

... better than last term... was actually more improving, getting onto work and doing it, not like last time. Last term or first term I start sitting there... but now I'm right straight involved. (interview, lines 156-162)

Lizzie considered Thor's improved attendance, engagement, resilience, and perseverance were evident on a daily basis. She described his changed behaviors in TFG3 and her interview:

Definitely seen an increased engagement... since we had that lunchtime meeting where we shared... his attendance has definitely improved and his effort, you know, his willingness... he pushes himself a bit more to attempt tasks whereas before he would have been a bit oh you know, and so he makes the effort to have a go. (TFG3, lines 267-270)

He's committed to his class work and his homework, which are all improvements... He tries and tries. He doesn't give up. Before, if he was finding something difficult he would just "Oh, I can't do it," whereas now he'll struggle with it and ask for help and push through that, and not just help from me but his peers as well so that's really great. He has taken a lot more care 
over recording his work because prior to being involved in this, often he might now bring his book in, it would be on bits of paper and this, that, and the other, whereas he always has his book now and he's got a good record of his learning. He attempts all the tasks now, whereas before it would be a bit of a, "Oh I'll do this, or I won't do that, sort of thing. (interview, lines 224-231)

Thor's report of improvements in other subjects as well suggested as he started to attend and engage more in learning activities, he discovered this was more fun than being passive in class, and gained satisfaction from learning successes. A positive spiraling effect seemed apparent:

Same as Maths. 'Cos I used to sit there from... oh I don't wanna do any work, and then now l'm doing all my work hard out now, it's like cool as, like fun. It's like way funner when you're learning not sitting there "cos you just get bored after a while... Yeah, at the end of the day, yeah neat, I learnt something new, and if you don't, just sit there and the day's just dumb and long... and then you get naughty after a while... Yeah, it's like, oh damn, better get onto my work. Trying to get them all correct, asking my mates for help. It's like "What's this answer?" and I was like "Hell, just give a clue and then I'll get it straight on." My Maths, it's like oh, fun as. (interview, lines 187-221)

However, Thor felt he wasn't "on his good side" in Visual Art. His comments suggest in this subject he had not gained self-efficacy:

All my classes are on my good side. I think it's... One class that I'm not good at working on, I think it's Art I think, 'cos I'm not really artistic, and you just think, oh damn, and then can't do it 'cos I get really nervous and I think, oh, sh... I can't do it, I can't do it. (interview, lines 241-244)

Thor seemed to have developed a greater sense of belonging at school and a stronger sense of identity as a learner. In almost all subjects, he reported greater self-efficacy, demonstrated effective learning behaviors, and was enjoying his own learning progression. Thus, I argue he developed a sense of his own agency as a learner, and was exercising agency in a way that was likely to lead to improved achievement outcomes. Reflecting back on Thor's hopes, articulated at the beginning of the study, I wondered how much he had yearned for an academic identity, as his words "go to university, then I could talk about this project" (reflection) hinted. Thus, it seems participation allowed Thor to become more successfully integrated in school life and learning, which had previously eluded him.

For Sonny Bill, who was already well integrated at school, changes in classroom experience were less pronounced. Lizzie reported prior to the study he had good attendance and "pretty good" (Lizzie, interview, line 315) commitment to classwork and homework. She observed Sonny Bill already had status and popularity with peers, which Ses affirmed, stating: 
He's pretty sociable, good looking boy, pretty good little group of friends and gets on well with a lot of people, so I don't think (his transition to secondary school) was too much of a challenge for him. (interview, lines 517-519)

Sonny Bill himself reported implementation of strategies had not changed how he felt or acted in Science, and Ses was not aware of any such changes either, although he believed he was the wrong person to ask. Nevertheless, Lizzie observed some improvements. She believed his commitment to learning had improved "not necessarily to the... 'cos his was always pretty good anyway, it's not to the same extent as Thor. But I suppose the big difference that I see is that ability to self discipline and refocus" (interview, lines 314-316). She believed his on-task time had improved, because although he got distracted at times, he now quickly refocused on learning. Other changes in Sonny Bill's behavior that Lizzie recounted included asking more questions and offering more ideas in discussion. Sonny Bill commented on one difference he noted: "I reckon it's made like class a working class more fun because I reckon, well I don't know if it's because of this, but it's quite fun work" (Hui 4, lines 77-78). One activity he considered enjoyable was within a unit about genetics after "Diego Run"; students brought in their baby photos and tried to match each to its owner. Apart from that, Sonny Bill was not sure that there was much difference in the classroom.

\subsubsection{Co-construction of learning with students}

In her initial reflection on hoped-for valued outcomes, and in Hui 3, Lizzie shared her desire to learn how to incorporate knowledge about students' FoK into lesson planning, and achieve positive impacts on learning. In her interview, she reported she was inspired to increase the scope of her pedagogical practice, relating a number of new ideas she had applied in the boys' Science class. In Hui 4 she confirmed the value of her new pedagogical approaches:

I just wanted to say that it's been great but I don't see it as an ending, I see it as a beginning, do you know what I mean? Starting. (lines 652-653)

Building on the collaborative planning for "Diego Run" with Sonny Bill and Thor, Lizzie extended this idea, applying it with the whole class. The next unit of work was "Extreme Beliefs." To maximize potential benefits, she conducted a class discussion to co-construct the unit plan before starting it:

So I shared with the students what my thoughts were as to how the science might fit in with extreme beliefs, 'cos I knew that they were looking at Nazi-ism in Social Studies, and they're going to look at freedom fighters in English... So I just shared my sorts of ideas and I said. "Looking at what l've got here and thinking about what you've learned in the other subject areas, what are the sorts of things that you want to know more about? Is there anything up here that l've shared with you that you want to know more about? Or if there's 
anything from your own personal interest around this area or with what you've learnt in your other subject areas that you want to know more about, just write them down. (interview, lines 340-348)

This proved productive, as many students contributed ideas, highlighting some relevant contexts for teaching Science concepts. Lizzie stated:

Heaps of the boys wanted to know about how bullets travel and guns, and how far would a bullet go through a block of wood and all that sort of stuff. What sorts of guns did they use in World War Two. And there's a heap of information there that provides a learning context, so we can look at forces in motion within that learning context. (interview, lines 351-355)

Lizzie stated the co-construction process was "great" (Hui 4, line 229) and expressed her intention to repeat it, because it was "valid for any teaching and learning from here" (Hui 4, line 229). She liked the way it allowed for possibilities to stimulate students' curiosity, which she believed was a strong motivator for learning. She also stated it would have been valuable to include Ses in collaborative planning, to contribute information about Sonny Bill's FoK. She related a new idea for drawing on a student's FoK:

Well just something that came to me recently is that we've been looking at, we started this whole genetics and the extreme beliefs ties in with Nazis and Al Queada... so we were looking at the genetics but I know that one of the students in the class is an identical twin so I thought it might be nice if she's willing you know just to share something about her experience of being a twin and what it's meant for her growing up. (Hui 4, lines 277-282)

Lizzie deliberately started the co-construction process by sharing her own thoughts with the class, as she "didn't want to make it too onerous for the kids. I don't want them to think they've got to come up with what we're going to be learning" (interview, lines 356-357). In the absence of further elaboration, I can only speculate about Lizzie's thoughts. However, I believe that in sharing some thoughts first, Lizzie aimed to provide a framework to enhance students' understanding of ideas that may be valid, thus tempering the challenge of co-construction. In team hui she was consistently receptive to students' ideas, and if she displayed this in class then students would have been encouraged to contribute. Nevertheless, a possible threat to full participation in a whole class brainstorm is the class size; Thor observed, from his experience in whole class settings, individuals can easily withdraw and be invisible, and thus the intention may not fully achieved. As Thor put it:

(if we had been asked to give our ideas in Science class) I think it would have been different 'cos you will walk in there and the teacher will go "Do you got any ideas?" (sic) and you'd be like "Oh, sorry Miss." And then she'd be like "Thor, where's your ideas at? (interview, lines 525-528) 
As well as continuing to seek students' input into planning, Lizzie developed other pedagogical ideas to apply students' diverse FoK. For example, students investigated genetic traits within their own families by interviewing family members, presenting findings as a family tree, whakapapa, or pepeha. With such activities, Lizzie hoped students could draw on their own experiences and relate this to their learning about genetics concepts. However, she was unsure whether this strategy "helped them get a good education or supported their achievement" (Hui 4, lines 339-340).

Because of her deepened understanding of Thor and Sonny Bill, Lizzie stated when she was planning, they "pop up in my mind, rather than just seeing a sea of students... because l'm more aware of them as learners and as people, primarily as people" (Hui 4, lines 97-105). Lizzie understood this as due to her stronger relationship with the boys, and compared it to making a birthday card for her son:

I suppose an analogy would be, like I made a birthday card for my son ... recently, and because I know him and I know him really well, then I can think about things that he would enjoy and that would make him laugh... because of the relationship, then that makes it easier yeah. (Hui 4, lines 110-114)

\subsection{Conclusion}

Team A functioned successfully, creating a positive, inclusive environment which promoted enhancement of relationships between participants. In Hui 2 and Hui 3 discussion of strategies by which Lizzie could learn about students' FoK progressed smoothly to genuine agreements. Inclusive practices such as rescheduling allowed full participation of all members. Therefore Thor, who sometimes had attendance difficulties, could be a committed team member on his own terms, and he enjoyed significant valued outcomes.

Implementation of agreed strategies resulted in team members seeing each other as people, enhancing teacher-parent and teacher-student relationships. Nevertheless, learning about students' FoK proved challenging. Lizzie considered focus and clarity of purpose were important in the work, and developed ideas about drawing on parents' knowledge to overcome students' modesty. Tensions between support offered by prompting questions and direction they can provide were identified.

Valued outcomes achieved related to participants' hopes recorded when the study began. Students stated Science was now "funner," and both demonstrated improvements in effective learning behaviors. Lizzie's original hope - to create more relevant learning experiences for her students, aligned with and integrating their FoK - was fulfilled. Involvement inspired Lizzie to implement ongoing co-construction of unit plans with students. New activities in the Science class allowed students to 
draw on FoK from their family experience and cultural knowledge. Because of her deeper knowledge of Sonny Bill and Thor, Lizzie's planning now included reflection on their perspectives.

Findings also illuminate teacher qualities (e.g., flexibility and openness) that supported valued outcomes, and skills (e.g., questioning) needed for successful learning about students' FoK. 


\section{Chapter 5: Team B Findings}

\subsection{Introduction to Team B}

Team B's teacher was Kate, a Pākehā teacher in her thirties who described herself as "fairly proficient in conversation" in Te Reo Māori. Kate had spent her entire eight years teaching service at KHS, and at the time of the study was in a middle management role. She taught Classical Studies, Social Studies and Te Reo Māori.

Team B's students were Dan and Troy, the only male senior students at KHS who participated. Dan, who identified himself as Māori, was fluent in English and Māori. When the study began, he was in Kate's Year 13 Classics class, and was 17 years and five months old. Dan said the statement that best described him in Classics was "I really enjoy it and it's going well." He had known Kate in her role as Dean for three years, and both he and Kate judged their relationship to be "very good."

Troy also identified himself as Māori. He was sixteen years and five months old when the study began, and he was in Kate's Year 12 Classics class, which was his first contact with Kate as a subject teacher. Later, Kate clarified before the study she did not know Troy very well, but she knew of him, due to his reputation as a student with a long, ongoing history of classroom behavior issues. Prior interaction between Troy and Kate was confined to one incident several years previously. Troy was in the hallway during class time and Kate called on his help, saying "I'm teaching Māori at the moment, I could use a bit of a hand" (Team B Hui 4, lines 260-261). When the study began, Troy was regularly removed from classes, had been excluded from several subjects due to disciplinary infractions, and often was on daily report. Being on daily report meant a senior staff member monitored his behavior on a daily basis, and Troy's subject teachers evaluated several behavior elements after each class. However, Kate managed Troy differently, refusing to remove him from class. The statement Troy said best described him in Kate's class was "I really enjoy it and it's going well." Both he and Kate categorized their relationship as "mostly good."

Tash, Troy's mother, was a Māori woman in her thirties, who worked fulltime in a professional role. When the study began, she reported an established relationship with Kate that was mostly good, describing their previous interaction as "neutral." Tash described how Troy carried the burden of his brother's reputation at KHS:

Probably l'd say before being on this project the only time I saw Kate was... whenever (my other son) was in trouble, and he was in trouble quite a bit... because (Troy)'s the younger brother he had the "Oh, you're just like your brother... and he's nothing alike, they're absolutely nothing alike. (PFG3, lines 290-302) 
Catherine, Dan's mother, was a Māori woman in her forties. She worked fulltime in an administration role. She stated she had a well-established, "very good" relationship with Kate, featuring collaborative sharing of information, both from teacher to parent and parent to teacher.

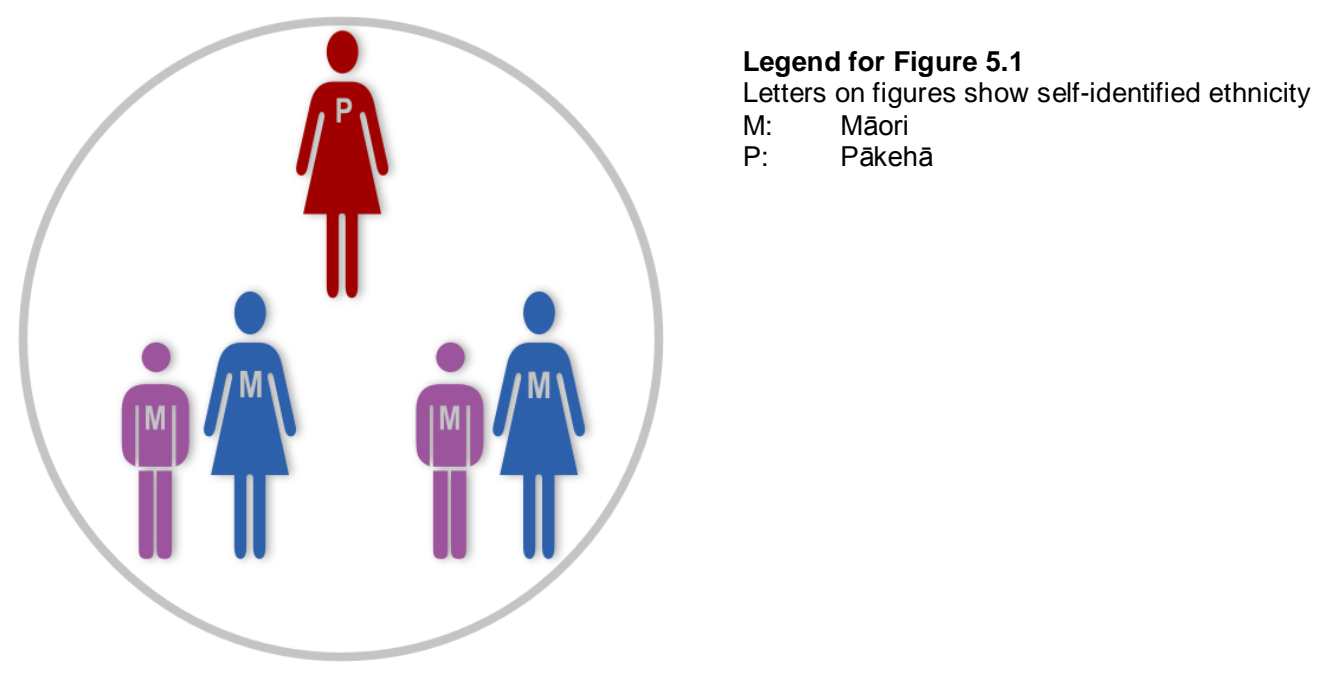

Figure 5.1: Team B participants

\subsection{Synopsis of findings}

Drawing on her prior knowledge of the importance of knowing Māori students in order to have an effective teaching and learning relationship with them, Kate took on a broad conceptualization of FoK. By the close of the project, she considered FoK included students' pedagogical FoK and preferences, things which affect their emotions, experiences, areas of expertise - things that make up the person. She summed this up as: "it's what has made this person the person that is in front of me" (interview, line 18).

Kate chose two senior students to participate in her team, hoping to support their learning more effectively through learning about their FoK. She reported "That's part of the reason I chose Troy, I knew he was going to struggle this year" (interview, lines 182-183). Writing about her hopes for the project, Kate wondered "What aspects/funds of knowledge have occurred in (Troy's) life to bring him to become the student/ learner who is in front of my class. Why do these barriers/ resistance appear? How can I create a better learning environment for Troy?" (reflection, Hui 1). Tash believed her son Troy was more confident in smaller groups, and hoped his participation would help him successfully complete the school year. Troy himself stated he wanted teachers to respect the way he wanted to learn. Because this was Dan's final year at school, his mother Catherine hoped the study would generate 
valued outcomes for future generations, by identifying ways teachers can "teach in a way that relate to the kids" (reflection, Hui 1). However, Kate hoped "to use my understanding of Dan's FoK to assist him in his learning and goals in his learning for the future" (reflection, Hui 1). Although Dan struggled to articulate clear hopes, he expressed the hope it would help him to learn, and hoped it would not involve "talking of it too much" (reflection, Hui 1). Later he added he would like teachers to acknowledge his FoK.

Team B parent and student participants brought to the study negative experiences of schooling (either their own or that of whānau members) and a range of relevant prior knowledge. These FoK affected several members' decisions to participate, and the nature of team interaction.

Team B teacher and parents formed a whānau around Dan and Troy for their support. They shared a deep commitment to listen to and respect the students' voices, and established a team environment in which the boys' goals, challenges, strategies, and opinions were paramount. The students were accorded equal power and status within the team as adults. The way Kate spoke with them also highlighted care for the students.

Agreement on a strategy was reached quickly in Hui 2, as soon as Kate, Catherine, and Tash realized that the students liked the idea of Kate observing them each doing a favorite extra-curricular activity. Although the students were absent, I conveyed their strategy preference. In Hui 3, a refinement was agreed, for Kate to observe Dan doing an individual activity. Also, Kate discussed how she could draw on the boys' FoK to support their learning; this was the aspect in which she lacked confidence. Challenges constrained the success of the process. When Kate approached other staff members involved with Troy's pastoral care to propose ways management of his behavior might draw on his FoK, they did not agree, so the strategy could not go ahead. When Hui 4 was held, teaching commitments had prevented Kate from observing Dan, but the problem was resolved when I provided support as a relieving teacher.

To progress project aims without team discussion or agreement, Kate also had a more private session for Troy, with him and a friend. Kate stated this time was "disguised as let's talk school, but actually was mainly... (a chance to) ask questions which I didn't know the answers to" (interview, lines 48-49).

Team B perceived significant valued outcomes were achieved. Troy and Kate developed a close relationship as a result of her learning about his FoK, and she became his major source of support for the remainder of the school year. Troy 
completed the school year and gained his NCEA qualification. Kate also developed her knowledge of Dan, and he also began to seek her support more frequently. Like Troy, Dan successfully completed his school qualification, including achievement of credits in Classics, for a topic Kate scaffolded by drawing on his FoK. However, both parents believed valued outcomes would have been more significant had Troy and Dan been involved in such an initiative earlier in their high school years.

\subsection{Evidence by theme}

\subsubsection{Preferred approaches for teacher to learn about students' FoK}

Team B's team agreement recorded during Hui 2 stated that Kate will:

observe Troy at an indoor netball game and Dan at Kapa Haka, to see how each boy operates as a team member. Then to follow this up by drawing on the team skills and knowledge within a class activity. (Team B agreement 2006-11.pdf)

After the two observations, Kate realized she would learn more from watching Dan in action as an individual. Focusing on Dan and his FoK was too difficult when watching him perform in the kapa haka group, because for Kate the group included "forty of my babies" (Hui 3, lines 82-83). Therefore she suggested observing Dan teaching kapa haka, a weekly activity in school time in which he coached a group of primary school children. Also, having learnt about Troy's FoK related to persevering, staying calm, and strategizing in netball games, Kate wanted to explore ideas about helping Troy to draw on those skills to support his self-management and learning in the classroom.

Therefore, in Hui 3, Team B decided to implement three further actions:

Kate will watch Dan teach his kapa haka group (during school time the following week). Kate will make a plan and meet with Troy to help him transfer his skills and strategies from sport to the classroom (by a date four weeks hence). Kate will try and get Troy's daily ${ }^{26}$ more personalized and change the staff member who's managing it, through correct school procedures (after the upcoming exam week). (Team B agreement hui 3.pdf)

\subsubsection{Enablers that supported valued outcomes in Team B}

\subsubsection{Team members' FoK}

Negative experiences of schooling combined with concern for future generations influenced motivation to participate for Catherine and Tash, giving them prior knowledge that supported Team B's effectiveness. For Catherine herself, and Dan's brother, high school was mainly a negative experience. Also, Troy's older sibling's

${ }^{26}$ Daily is another name for daily report 
high school experience was predominantly negative, and Troy himself was barred from enrolment in various courses due to behavioral issues. These dark FoK (Zipin, 2009), knowledge about negative schooling experiences, significantly influenced contributions, providing the basis for much candid discussion.

Tash and Catherine were also both confident and aware of their value as team members, in terms of knowledge they brought and the support it provided their sons. Tash also stated "I like being a part of things... if I have something to say l'll say it and if it can help future students all the better. So I like building foundations... in something good, something positive" (interview, lines 95-108). When the study began, Tash was beginning to advocate for Troy at school, to create a learning pathway into a career. Her professional work also related to advocacy and support.

Both students had experience interacting in adult forums and environments. Dan attended kapa haka meetings, which he found similar. Most of Troy's interaction outside school was with adults. He participated in adult groups, such as his netball team, confidently and on an equal footing.

In Catherine's interview she explained her experience at school was captured by the saying 'children should be seen but not heard':

That's what it was like when I was going to school - nobody gave a damn about my opinion, nobody thought my opinion was useful, and to be honest none of my teachers even gave me the chance to have an opinion. That's why I think it's so important, and then if you are in a meeting like we were, we all go in as one. It's not like Dan's the student so his opinion means less small (sic) just because he's a young kid, and it shouldn't be that Kate's opinion's valued more because she's a teacher. You're all on the same wavelength. (interview, lines 300-306)

To her, the most important issue in schooling was for students to feel valued.

Catherine was willing to participate due to her trust and respect for Kate. This came from years of seeing her at events which were important to Māori students and families, knowing about Kate's respect and love for Māori culture and language, and from dealings with Kate in her pastoral care role. Catherine, like other students and parents, called her Whaea Kate. The Māori term whaea means mother. It indicates Kate's involvement in the KHS whānau unit, and relates to the Māori notion of teacher as mother, and class as whānau (family). Catherine stated:

Once I knew that Whaea Kate was going to be in our team, the whole thing just changed for me... I feel very relaxed and very confident, because at the end of the day I know she's got the kids' best interests. (PFG1,10m.43s$11 \mathrm{~m} .09 \mathrm{~s})$ 
(If it was a teacher I had negative feelings about), I probably wouldn't have done it. I would have said no straightaway... I probably would feel that we would just clash all the time. And then for me, it would just be going nowhere. (PFG1, 13m.51s-14m.28s)

Tash would have considered participation with a different teacher, but the prospect of working with Kate helped her commit to the project.

Although not Māori herself, Kate had strong prior knowledge in Te Reo Māori and tikanga Māori, and of research evidence relating to effective education for Māori students. Kate's immersion in Māori ways included, for instance: fluency in Te Reo Māori; teaching in the whānau unit; and her self-identification as "a Māori leader" (TFG3, line 27). Kate had facilitated E Tipu E Rea TPL several years earlier, and "embedded" (TFG3, line 11) knowledge about factors creating effective learning for Māori students. This contributed to her belief "I can teach well in my classroom because I know every single one of (the students)" (Hui 4, line 397-398). She joked about herself as a teacher who would "stalk" (TFG3, line 282) students, meaning she would follow up absences or problems, taking notice, and providing support as needed. This was her professional style. Thus, the study aims and process were congruent with Kate's beliefs and practice. Therefore, as Dan stated, being in the team did not change anything, because "she was there for the group, she was there for me, and especially for the school, she helped me through it; even though it was hard for me, she helped me; it hurt my brain, she still helped me" (interview 5m.45s6m.06s).

\subsubsection{A new dynamic for interaction: Whanaungatanga at work}

The nature and function of the team, as well as the nature of communication within Team B, created an opportunity for a new form of interaction, which related to principles of whanaungatanga, directly generating benefits for members.

Two features appeared to influence the relationship dynamic. These were Kate's way of talking to the students, and the focus in discussion on the boys' opinions, ideas, goals, challenges, and strategies for moving forward. Catherine, Tash, and Kate explained the study's timing late in the boys' school lives, combined with pressure from NCEA requirements and examinations, increased the urgency and purposefulness of the work. There was a sense that time was running out for both Dan and Troy.

Kate was honest and direct in all her communication, including expression of perceptions and feelings. Early in Hui 2, Kate clarified her aim for the team: 
Kate: I think we can almost go that next step, and looking at their learning, and how I can get these boys through this year. I mean that would be it for me, to get these boys achieving.

Catherine: I mean how far are you willing to go?

Kate: $\quad$ I want these boys to pass, and I want Dan to get out in that big world. (3m.09s- 3m.24s)

At the close of fieldwork, in her interview, Kate reiterated her original idea, stating "we all met and let those boys know that we're a whole family behind you... and I'm a family member too, you just see me for five hours rather than at home... and I loved that part of it" (lines 168-174). Some examples that illustrate Kate's communication style are presented in Table 5.1. I would argue this approach highlighted several key messages to the students (stated in my words): We are all adults; I care for you as an individual and as a learner; I am committed to supporting you; being realistic and honest will help us identify how to move forward; you have expertise relating to yourself and your situation.

Catherine posed questions to draw out Troy and Dan's goals, challenges, and strategies, particularly in Hui 3. Examples were:

- What year are you? (line 326)

- What are you learning about? (line 358)

- How's your Māori going? (line 388)

- What's your career path, son? (line 636)

- Have you got it written? (line 738)

Therefore, Catherine appeared to actively draw out student voice, believing children should be heard and valued. As she took this leading role, others seem to have also taken up this culture, including myself. Other questions posed in Hui 3 included:

- What's the subjects you're taking, Troy? (Linda, line 334)

- Is that the kind of thing you'd be interested to do when you leave school? (Kate, line 370)

- How many credits do you need? (Troy, line 752) 
Table 5.1: Examples of ways that Kate talked to students

\section{Description Examples}

Feelings towards I've watched you as one of forty of my babies... there's 39 others that I love just as much (Hui 3, lines 82,106 )

students I was really excited about the

fact that I was going to see him (Hui 3, lines 125-126)

I was really sad I didn't get to see you Dan (Hui 4, line 3)

Care for students It's nice that you want to pass Classics, but I want you to pass Level Three (Hui 3, line 308)

as learners The two boys got through their internals, which is what I wanted (Hui 4, line 47)

Tough love l'll be sending mum a timeline, so mum will know when those opportunities are... so therefore when they're not being taken up... (Troy sniggers) You're the same, buddy... don't think you're getting out of this, you've got an internal coming up too (Hui 3, lines 281-290)

Direct, honest statements about

the students

You knew you would be there in five steps, and no bigs, and you would bat the ball away, and off you'd go, trot, trot, trot. Whereas your confidence levels of your abilities in the classroom are not there at all, and it's transferring them through, without you getting angry at yourself and sulking (Hui 3, lines 219-225)

Dan doesn't love Classics (Hui 3, line 302)

Let's look at the bigger picture of how do we get NCEA Level Two, and how do we get you not having to repeat, 'cos at the moment l'm fearful (Hui 3, lines 314-316)

I'm just thinking, last three times l've left you with a reliever, Troy, you've nearly got stood down (Hui 3, lines 889-890)

Own perspective When he said it was a six o'clock game, oh sweet. If he'd said nine o'clock, I might have been a bit more..." (Hui 3, line 193)

Direct, honest

statements about

teachers or school

practices

Yes, yours probably will, but no, that's not fair. The criteria should be the same for all students... yeah but you probably will be asked about your behavior Troy (Hui 3, lines 455-457)

Miss Smith would be a good person for you to work with... she's really down to earth, and would do really well with you... don't do it with me, 'cos everyone knows I stick up for you too much (Hui 3, line 528-529)

I don't know if he's going to let you go very easily though, let's be honest. He's probably a bit stressed, too (Hui 3, lines 826-833)

He doesn't have to see Troy for four days, so he might be quite joyful, no offence, sweetheart, but... (Hui 3, lines 920-921)

Teachers get really feral at the end of the year when kids don't come to their last week of classes (Hui 4, lines $80-81)$

Questions promoting student

I mean, when you have your sulky... what can we do to get you out of it?... Do you have strategies where you just walk away, rather than reflection

Do you know... how you're going to do it? Have you got a timeframe?... Do you need someone to stalk you?... Or just leave you be? (Hui 3 , lines 729-738)

Who are you more comfortable with? Who's best? (Hui 3, line 812)

Advice

You should go to a Year 13 student who got interviewed at the beginning of last year and ask them (Hui 3, lines 450-451)

If you reflect, teachers love reflection. Love it when you can say I realize that in the past I have blah blah blah, and these are the steps that I would like to try and do to look after my own behavior (Hui 3, lines 462-463)

Choose one or two... and just do them hard out (Hui 3, lines 692, 698)

Come in on the Wednesday when you've had your sleep in or whatever, and you can spend some quality time (Hui 4, lines 75-76) 
An excerpt from Hui 3 is shown, as an example of a conversational thread:

Catherine: Have you got it written?

Kate: Are you okay with it? Have you got a time plan?

Dan: Yes, it's going to be hard. No, I have no good time plan.

Kate: Do you need someone to stalk you?

Dan: If you want, Whaea, yeah.

Kate: Or leave you be?

Dan: No, I might need some help.

Troy: How many credits do you need?

Dan: A lot. I'm just trying to keep it in my head.

Kate: It's doable.

Dan: I know how to get it.

Catherine: $\quad$ Mr Henare ${ }^{27}$ said if you did your (credits in Māori Performing Arts), you can pass, so you need to pull your finger out of your bum, as (he) would say, and get onto your MPAs, son.

Dan: I know, that's why l'm doing the school thing, teaching students.

Linda: $\quad$ The good thing about writing all this down is that, next to each goal, you can write down what you're going to do to achieve it, or when exactly, so you can start to plan out how you can make it happen, and that helps it get closer to reality, 'cos you're making, like a plan of attack.

Tash: $\quad$ And then you can call on the resources, like the teachers. (lines 738-769)

Troy and Dan could relate as equals, because they had power and status, and their ideas were valued. For instance, the strategy agreed in Hui 2 was favoured because it was their preference. Catherine knew as a parent decisions made without children's involvement could create problems and leave them feeling powerless: "Oh man are they talking about me? What the hell are they doing?" (Hui 4, lines 509510). Thus, she saw the students' active agreement and participation as essential:

At the end of the day it's about them you know, so they need to be involved, and they need to have their input as well... I think it's quite important to have them involved too as well, so that you know we're not doing this research, even though it's for them, we're not doing it behind their back sort of thing... we are including them face to face and it gives them a chance to like step up. (Hui 4, lines 523-528)

Nevertheless, despite these stated beliefs and intentions, a combination of various factors resulted in other members' voices being more prevalent in team hui. Dan stated he enjoyed the opportunity to suggest and discuss ideas. However, he also

27 Pseudonym for another teacher 
found it hard to find a space to speak, and expressed frustration with his mother's enthusiasm for discussion:

Me and Troy, we pretty much just looked at each other while our mums talked, and we're like, shall we say something?... Once I say something, my mum will be like, she'll jump onto it, she'll jump onto it, and l'm like, oh, can't even answer the frigging question. (interview, $9 \mathrm{~m} .25 \mathrm{~s}-9 \mathrm{~m} .42 \mathrm{~s}$ )

Unlike Troy, Dan also found it difficult to disagree with his mother.

The students' role and status in the team was highlighted again in Hui 4, which Troy did not attend due to a sporting commitment. Discussion related to the opportunity for learning through participation offered by the study:

Catherine: Like for them it will be that we actually value their actual opinion, do you know what I mean, they're not just teenage kids...

Kate: $\quad$ And I suppose it's modeling the system to them as well, about how adults, the adults involved in their lives at school, community and home community interact together in a hui without it being a formal parent-teacher interview.

Tash: $\quad$ Yeah discussing them

Linda: $\quad$ Yeah a way of interacting for their benefit. What do you think Dan?

Dan: $\quad$ Yeah good... if Troy was here he'd say the same thing. (lines 547-560)

Later, in his interview, Troy stated "It was cool... just doing it as a group, it was cool" (lines 5-9). Tash explained how the team interaction created a different dynamic:

He's thinking well that's my mum, that's my teacher, I'm always told what to do. When he's part of a group and working within a group he's an equal so I think he really respected that. I think he felt really comfortable being in that environment, but that's how I viewed him as being in the group... so the dynamic has changed instead of him just being a student being told what to do, his input was actually, whatever he suggested or his input, his thoughts, were treated as a good idea. (interview, lines 33-56)

In her view, this made Troy "think outside the square" (interview, line 29), which led to his "opening up to Kate... otherwise it's always been a teacher/student relationship" (interview, lines 42-43). Kate believed the team interaction "helped those two boys to grow a bit" (TFG3, line 52), affirming Tash's idea that it resulted in Troy stepping up into an adult role with his teacher. Troy's words confirmed Tash's impression of his appreciation of the team, but his brevity means interpretations must be tentative.

I argue three elements - Kate's ways of speaking to the students, deliberate surfacing of students' goals, challenges, and strategies, combined with clear 
devolution of respect and power to the students - collectively laid the groundwork for establishing whanaungatanga in the team, outlined in Figure 5.2. Therefore, the team interaction and environment was aligned to important principles associated with the participants' culture. Whanaungatanga is a complex Māori concept, encompassing ideas about kinship and principles that should be upheld in relationships to honour each individual's mana and support attainment of potential.

The concept of whānau (family) is central to whanaungatanga, because Māori define themselves in relation to their whānau, hapu (sub-tribe) and iwi (tribe). Although the concept of whānau relates to bonds of association and obligation arising from blood ties (Ritchie, 1992), other groups sharing strong bonds (e.g., arising from common interests, goals, and working together within a setting) may operate as a whānau (G. H. Smith, 1995).

Figure 5.2 summarizes concepts and principles within whanaungatanga, as conceptualized by Team B members. The following discussion draws links between these elements and data from Team B described earlier.

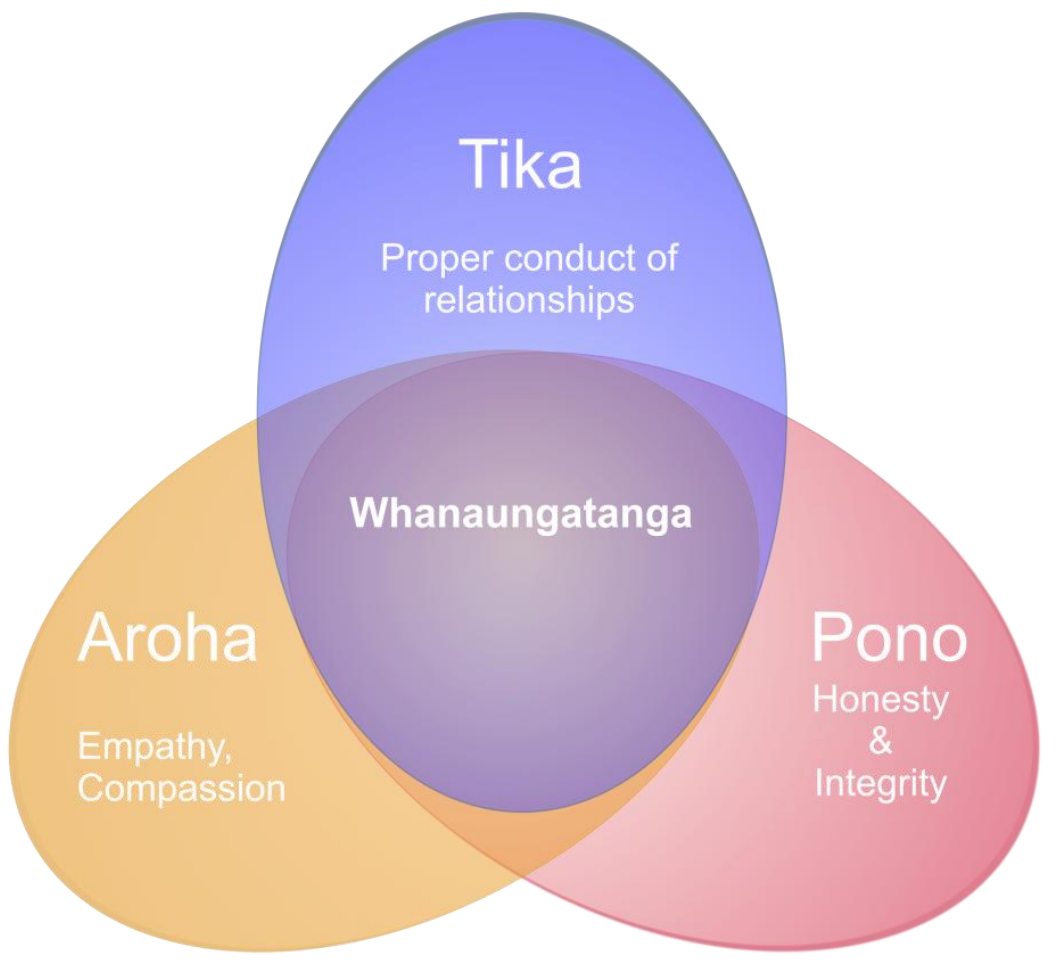

Figure 5.2: Elements of whanaungatanga

With her honest and direct approach to communication, Kate's actions were in accordance with the principle of pono, which calls for honesty and integrity in communication, to identify and address reality. Tika was also demonstrated, in her care for Troy and Dan as learners. Catherine's questions to draw out students' goals, strategies and challenges also demonstrated pono, showing empathy 
(aroha), thus honouring the students as sacred taonga (precious treasures). Troy's and Dan's mana was honoured by the respect, power, and status given to them, by the team process and attention to their goals and needs. Their mana was also upheld by exercising agency to affect change and self-development - through thinking outside the square and growing through the experience.

\subsubsection{Choice of strategies}

Team B's selected strategy of Kate observing the boys in a favorite activity appealed to the team members immediately, and retained appeal for members when reflecting back. The boys were "chuffed that the teacher was taking an interest, coming to see them in their space" (PFG1). Dan recalled "We were just looking at each other, and like yeah" (Hui 4, line 538). Tash and Kate both commented it was valuable to see the students "outside of the school environment away from other students, you get to see who they are and what they're passionate about" (Tash, interview, lines 321-323). Kate stated this was a great way to give them personal attention. She said "With Troy, I was really excited about the fact that I was going to see him... in a team that was just him and others I didn't know" (Hui 3, lines 125-127). Tash agreed, "You want to see him as an individual, and I think that's the thing" (Hui 3, line 137). Kate perceived in these circumstances "there's no front there, there's pride" (interview, line 718). Catherine also considered the strategy was appropriate to learn about her son, because "that way she gets to see Dan and what he can do hands on, and... he tends to be a lot more better hands on... whereas if he was to talk about it he might get himself a bit confused" (interview, lines 377-384). (New knowledge Kate gained about the boys is discussed in Section 5.3.4.1.)

A further aspect of this strategy not commented on by team members was its "doability" for the students. The strategy simply involved students performing their usual activity, with Kate watching. Thus, it was not onerous for the boys, and required no organization or work effort on their part. On a number of occasions it was apparent that neither boy had excellent organizational habits, as shown by Kate's remark in Hui 3: "Boys, you don't even think about what happens tomorrow, let alone what happens in Term Four" (line 1151), and the mothers' practice of reminding their sons about upcoming events. Therefore, I argue this factor also enhanced the strategy's suitability.

In Hui 3, Kate suggested two ideas to apply the boys' FoK to classroom learning. Firstly, Kate explained her idea to scaffold Dan's learning about Roman warriors, which was immediately agreed: 
So the way I thought l'd do it with Dan... is that we'd actually do it in two lines. So actually discovering a Māori tomb first, and attaching stuff for a Māori warrior, and instantly matching that stuff with someone in Rome... and all he has to do is mirror it completely with someone in Ancient Rome. It'd be so much easier. (Hui 3, lines 565-571)

Dan later recalled "She just gave me a sheet, and I followed every question" (interview, $4 \mathrm{~m} .10 \mathrm{~s}-4 \mathrm{~m} .18 \mathrm{~s}$ ). I inferred he was able to complete this independently, whereas for other aspects of his learning in Classics, Dan talked about how Kate guided him through tasks. He confirmed it was helpful to "look at both (the Māori and Roman warrior)... the Māori one was pretty easy" (interview, 4m.40s-4m.45s).

Secondly, Kate reflected on how Troy's FoK could help improve his classroom learning experience:

For you, it might be more about management of self, and bringing in those kinds of things regardless of the task - it's more about bringing in management of yourself. (Hui 3, lines 577-579)

Tash and Troy were enthusiastic about Kate's idea of individualizing his daily report and having it managed by a different staff member. However, the idea was vetoed by the staff member to whom Troy currently reported. (This constraint to valued outcomes is discussed fully in Section 5.3.3.1.)

As the selected strategies were popular with all team members, potential issues relating to reaching agreement and implementation were minimized. In team hui, when the strategies were initially suggested, others immediately perceived the value and took up the idea. For instance, the following conversation occurred during Hui 3 :

\footnotetext{
Kate: $\quad$ What I was thinking about is, is when you're teaching those kids, that fifth spell on Wednesdays, just you and George.

Dan: $\quad$ Oh Tuesdays.

Catherine: $\quad$ Oh yes, when you go down to Huia Primary School.

Kate: $\quad$ Yeah, I wondered, that might be a little more, it's just you and someone else, and it's all about you teaching, and

Dan: $\quad$ Yeah

Catherine: It's just a bit more personal. (lines 86-98)
}

As well as implementing agreed strategies, Kate also spent six hours with Troy and his friend during school holidays, ostensibly for tutoring. She reported "they spilled so much to me about their lives and about what's happening and things they probably shouldn't have done in the weekend that mum will probably never know..." (interview, lines 141-146). She believed the boys' openness was "because I'm not going to go and ground them at home" (interview, lines 141-142). 


\subsubsection{Researcher role}

Kate expressed appreciation for various aspects of the researcher role. Firstly having the project set up by another person, with her input regarding student participants, made participation easy. Her heavy professional responsibilities meant my administrative support was important to her. Secondly, Kate valued having a collaborator who shared her goals and care for the students:

I needed that, that's what I wanted, I didn't want to do this all on my own. And you could just tell that you cared just as much about what my boys were doing as I did and their parents did. Yeah, I knew, even though yes you were the facilitator, and et cetera, you were in that role, I always felt that you cared just as much about those boys as well. (interview, lines 890-894)

Thirdly, Kate found my facilitation helpful, because agreed timeframes were honoured. Although traditionally hui discussion lasts as long as it takes for everyone to express themselves and reach agreement, Kate stated it was useful "having someone just to guide back, or, especially with Māori as well, 'cos we can just go to tangents" (interview, lines 512-514). Lastly, my willingness, availability, and suitability (through teaching qualifications) to supervise her class enabled Kate to observe Dan as agreed.

\subsubsection{Barriers to valued outcomes in Team B}

\subsubsection{After four years: No more chances}

Team B's agreed strategy of personalizing Troy's daily report was not implemented, because when Kate presented the idea to the people who would be involved, they did not agree. Discussing challenges encountered when promoting the new idea, Kate stated:

We've got to remember that people have been backing him for four years and it's tiring and they're tired. I'm just new and fresh-faced, and let's focus on Troy. People are like I've been focused on him for three years and he hasn't changed. Why do you want me to do it again? And that's understandable... I just went fair enough. (interview, lines 630-634)

One staff member Kate approached was her close friend, and Kate was reluctant to "push it" (Hui 4, line 204) with her. Therefore, as Kate promoted her idea to colleagues, she also needed to manage her working relationships with them. Kate's words demonstrated respect for her colleagues' prolonged efforts with Troy. She perceived the considerable energy invested in their work with him, and their sense of exasperation, indicating any reserves of goodwill towards Troy seemed to have run dry. Although Kate acknowledged the approach to managing Troy over four years had not achieved desired results, she felt it was inappropriate for her to challenge her colleagues' approach. She stated: 
I can do it in my class and I can work with him and assist, try and get him to use some of the strategies that he uses in an environment amongst adults. But I don't think that I can help other people to help him use them. I don't think people are open to seeing that you can actually work with Troy and it's how you... people's fuses are shorter than others. And also... in a lot of classrooms, his behavior, his first behavior he would have been removed, whereas I'm just trying to get past that first behavior and yeah fair enough teachers have got every right to kick a student out when he performs like he does... I don't feel like I'm in a position to tell people how to do it 'cos he's not easy. (interview, lines 75-88)

However, Tash's view of the situation was different. She was "really really disappointed that they actually put barriers up there" (interview, line 147). She felt agreeing for the study to be conducted at KHS created an obligation to back it fully by supporting initiatives that arose from it. Therefore, Tash was disappointed by the lack of support for the team's suggested initiative. Similarly, Troy's initial positivity was replaced by cynicism. Catherine shared their frustration, stating:

It's really annoying, eh? They should be more open... he is doing this project. So they should actually see it as something positive... so they should give him the benefit of the doubt... I know kids can be naughty and stuff like that but at the end of the day you're a teacher, you've got to keep on trying. (PFG3, lines 477-487)

A further factor affecting Kate's efforts to personalize Troy's daily report was her realization that, "he wasn't even on daily at that point. And I thought by forcing Troy to go back on another daily l'm like..." (Hui 4, lines 154-155). However, Tash stated "a lot of the times when (Troy) comes off it, he requests to go back on it" (Hui 4, lines 159-160), suggesting he may have found it useful to generate focus. Thus, in this instance, Kate's view may have been not fully informed.

\subsubsection{Time and timing}

In several different ways, time and timing negatively affected Team B outcomes. Most importantly, Catherine and Tash considered it would have been valuable for teachers to learn about their sons' FoK much earlier in their high school lives. They believed that had this happened, their sons' relationships with teachers would have been better, and teachers would have been able to motivate them more effectively, and relate learning to their interests. Hence, despite benefits, in their view the project came "a bit too late" (Tash, PFG3, lines 420-421).

Due to the lengthy recruitment period, the team process began in June, almost halfway through the school year, and was completed in November, just before Troy and Dan left school. This factor compounded the sense of lateness for them.

It took time to implement strategies due to Kate's competing responsibilities. For example, after Hui 3, report-writing slowed down the implementation process. Also, 
because fieldwork needed to be completed within a school year, the pressure was on to implement strategies and identify outcomes within a short time period.

Finally, the passage of time proved to be significant after Kate observed Troy playing netball. In the following week extreme weather conditions were experienced in Wellington, a consequence of which was that after observing him, Kate did not see Troy until a week later. At that time, she noticed:

He was irritable and badly behaved in class; she eventually asked him to leave after he threw something and it hit another person in the eye. When they talked, she realized two things: one was that she had not spoken to him since the netball, not made any comments about it since that night, which she believed was contributing to his foul mood. (journal, 20-08-11)

This incident suggests that when teachers learn about their students, prompt feedback needs to respectfully acknowledge what has been shared. I reflected:

What this shows is that Troy perceived that Kate as his teacher had a responsibility to follow up on the indoor netball game that she observed, after that night. Before they talked and cleared the air, he was feeling ignored or neglected, because the viewing itself was not sufficient. (journal, 20-08-11)

\subsubsection{Valued outcomes achieved}

\subsubsection{New knowledge of the students}

Because of Kate's different levels of knowledge of the students at the beginning of the project, she was able to make bigger gains in her knowledge of Troy. Kate's ideas about how she already knew Dan, and information that would be useful for her to know, also affected knowledge gained. Although Catherine stated "I found out that she didn't really need to know any more about Dan because she knows Dan very, very well... she knows Dan inside out, she knows his ups and downs" (PFG3, lines 281-282), Kate did gain new knowledge about Dan's FoK. Through observing him teaching his kapa haka group, Kate was able to see what Dan and his co-coach had taught the group - thus affirming his kapa haka knowledge - and see qualities and skills demonstrated as Dan taught:

He has to get them in lines and you now you're talking about five to ten year olds who don't stay in line, they don't do anything you want... He's very calm, he was very, very calm, yep. Yeah, quite a good teacher actually... giving little looks ssh you know. (interview, lines 746-751)

Dan also considered Kate enhanced her knowledge of him, saying, apart from his Māori Performing Arts teacher, she was the first teacher who watched him coaching. Therefore, she saw aspects of him that were not readily visible. He appreciated Kate's acknowledgement of his FoK after the observation. He considered he was "a 
good, understanding leader" (interview, 11m.36s), and Kate's feedback affirmed his expertise and her understanding of his FoK.

Reflecting back, Kate commented she had invited Dan to participate to avoid a "massive learning curve" (interview, line 36), and later considered that due to her prior knowledge, she may have "chosen the wrong boy" (interview, line 41). However, she considered the focus on Dan had allowed her to learn more about him.

Tash considered it promising that Kate recognized her own lack of knowledge about Troy at the beginning of the study, because that showed that Kate did not simply accept the common view that Troy was a replica of his brother.

Kate stated she had "learnt a lot" (interview, line 44) about Troy from her observation, because "he was certainly different from the classroom" (Hui 3, line 151). As she explained in Hui 3 :

On the court, you could tell you were thinking, five steps before something has actually occurred. So you are planning these steps over here, and I kept thinking, why is he hanging back? Get in there! And then I realized, it's because you've already thought about what's going to be happening in two minutes, and you're right there already!... Also the fact that you had the confidence - the fact that you knew you would be there in five steps, and no bigs, and you would bat the ball away, and off you'd go, trot, trot, trot. (lines 202-220)

Catherine and Tash agreed Kate's knowledge of Troy had increased. Tash affirmed Kate's ideas about Troy's FoK. "She was spot on... he does think two or three steps ahead... he could be analytical, he could be strategic" (interview, lines 339-344).

Kate's learning about Troy was not confined to his FoK as defined at the beginning of the study: knowledge and skills arising from lifeworld experience. Kate's conceptualization of FoK incorporated other factors that influenced a person's identity. Consequently, she also sought information related to Troy's relationship with his absent father, as well as "his temperament, about his personality, what pushes his buttons" (interview, line 57-58). Describing what she was trying to find out, Kate said:

It wasn't about learning about their past or things in their lives that then I use practically in a classroom, it was more about who are the men that are coming into my classroom and how can I get them to learn better? (interview, lines 346-349)

\subsubsection{Improved teacher-student relationships}

A bond developed between Troy and Kate over the course of the study. Kate stated "the fact that we, me and him are where we are is because of this, is because I chose to focus on him, yeah, and the relationship is good because of it" (interview, 
lines 92-93). Troy confirmed that his relationship with Kate "just got close" (interview, line 29). Catherine commented, "seeing her with Tash's son, it's something new and she's actually bonded to him as well. She's actually a lot closer with Troy now, I've noticed" (PFG3, lines 286-288). Tash agreed, "Yeah, and I think they've actually got a connection going on" (PFG3, line 311).

Troy developed greater trust in Kate, and began to seek her support for his challenges at school beyond Classics. Kate's classroom became a safe space for him at school when needed. Kate stated:

With Troy, if he's been kicked out of another class then he will just come down to (my classroom) and sit or do whatever. (interview, lines 234-235)

When he's angry and frustrated I'm the first person he comes to. (TFG3, lines 160-161)

Tash confirmed she observed Troy "opening up to Kate" (interview, line 42).

Correspondingly, Kate increased her support for and commitment to Troy. She stated "I think I would have classroom moved him (sic) a number of times, but this has made me care about him, it's made me look out for him, and have his back when he needs it" (TFG3, lines 152-154). One way this manifested itself was Kate's new practice of looking out for him, not just in Classics, but also in other areas of his school life. Tash stated Kate was a great support to Troy, resulting in his "knowing there's a teacher with his best interest at heart and keeping him on track" (interview, lines 23-24). Troy reported Kate now "expects more" (interview, line 43) from him in Classics class. He confirmed Kate gives him tough love, which works for him. After the six-month-long fieldwork, Tash observed "I think he's changed quite a bit especially in the last six months, more about he's more comfortable with him (sic), and that's what you've given him" (Hui 4, lines 303-305).

Tash considered the entire team provided a support structure for Troy: "Troy had a support mechanism, not just within a teacher, but within another student and within the parents" (interview, lines 132-133). Troy himself stated that he had become closer to both Catherine and Kate, "his mum and Kate, we weren't as close" (interview, line 21), suggesting he too valued support from others apart from Kate.

Although Kate knew Dan very well before the study, she also observed benefits for their relationship. The study resulted in her increasing her focus on Dan. She stated:

I think I really enjoyed building that close relationship with Dan 'cos usually he's one of many in the whānau unit. And I don't think I would have taken the time necessarily to meet with Dan 'cos there's other more dominant creatures... in his class... Yeah and it was about him rather than his... brother. (interview, lines 207-213) 
Dan also alluded to this when he said:

We (Kate and I) mostly don't talk to each other, 'cos my mates in my class, she's mostly immune with them, instead of me... she knows me and my mates real much, but she mostly hangs out with my other mates... she knows me real much, she's in the whānau unit. (interview, 1m.10s-1m.45s)

Like Troy, Dan began to seek Kate's support more:

It means they now come to me when they need to just sit, and often it's just can I sit in here? And we don't say a single word at all, it's just, I just need to sit here and that's it. (Kate, interview, lines 220-222)

With Dan lately it's been can you help me with looking at some courses at Polytech and things like that... it's about all facets of life. (Kate, interview, lines 235-237)

Troy and Dan sought Kate's help for assignments for various subjects and to talk about issues in their lives, taking the initiative for one-on-one conversation as Kate had previously, to the point where, as Kate said, "I don't seek them out any more" (interview, line 231).

Kate considered participation in the study provided her with a structure that allowed her to spend time with the students, and "given me a great way to get relationships with them" (TFG3, lines 51-52).

\subsubsection{Successful completion of the school year}

With Kate as well as his mother Tash backing him, Troy successfully completed the year, and gained the qualification he was aiming for: NCEA Level Two. Kate considered Troy's achievement level had been enhanced by their participation in the study: "Through that he's gained nine internal credits and will get his externals with me, I don't think I would have done that with him" (TFG3, lines 154-156). Tash summed up the positive outcomes for Troy:

He got to make it through to the end of the year. He had a teacher who every step of the way was focused on his best interest and making sure what he wanted to do at the beginning of the year was achieved by the end of the year. He wanted to achieve Level Two, he's done that prior to before the exams had even started, so for me that's a win. (interview, lines 16-19)

Tash and Kate both agreed Kate's support had been necessary for Troy to complete the year:

Tash: At the end of the day l'm really really glad we've been a part of this as well because I think if we weren't a part of this I don't think Troy would have made it through the year.

Linda: Oh really? I'm really glad you have.

Tash: Yeah and it's being a part of this here and having that bond that I think has got him through. 
Kate: It was a fresh face, someone new battling in his corner, and I think he just needed it for the rest of the year.

Tash: Just for this year and I think it's worked, yeah.

Kate: $\quad$ Someone who still wants to stalk him. (lines 717-727)

Furthermore, Tash reported Kate became an advocate for Troy, because she "actually helped me with the other teachers, saying, 'well, he's not (his brother), don't treat him like (his brother), he's Troy, he's completely different.' So she helped me a lot" (PFG3, lines 316-317). In her final interview, Tash restated that Kate was "keeping other teachers informed of him and his outside personalities" (line 24-25), suggesting Kate was comfortable with sharing her new knowledge about Troy, in contrast with her discomfort regarding raising questions about colleagues' approach to management.

\subsection{Conclusion}

Team B members had significant FoK related to negative schooling experiences. For most, their trust in Kate, who was known for her respect of Māori culture and fluency in Te Reo Māori, was a key influence in their decision to participate.

The teacher and parents formed a whānau around Dan and Troy during their final months at school, exploring how a focus on their FoK could support achievement of their academic goals. A team environment was established in which the boys' goals, challenges, strategies, and opinions were paramount. Thus the team supported the students, and also provided a space where they could take on a more adult, agentic role in their own education. The team culture can be characterized as whanaungatanga.

The team decided Kate would conduct observations of the boys, according to their preference. Individual observations of the students in unfamiliar settings, in which competing demands were minimized, were highly appropriate for learning about Dan and Troy's FoK. Kate already knew Dan very well, but she gained new insights into his FoK. At odds with his tempestuous and disruptive behavior at school, Kate's observation of Troy provided insights into his considerable strategic and selfmanagement skills.

Participation allowed Kate to implement a greater focus on Dan and Troy as individuals. As a result of learning about their FoK, Kate designed a task that highlighted Dan's FoK, to scaffold his learning in Classics. However, her attempt to implement a new approach to Troy's behavior management that drew on his FoK was vetoed by colleagues. The bonds between Kate and both students strengthened. Secure in the knowledge of Kate's love and commitment, both 
students began to seek her support more frequently. Both Dan and Troy gained their NCEA qualification at the end of the school year.

Kate valued my establishment of the team framework and administrative support, which made the work manageable for her. 


\section{Chapter 6: Team C Findings}

\subsection{Introduction to Team C}

Team C's teacher was Georgia, who identified herself as NZ Māori. She had taught at KHS for the entire length of her 15-year career. Georgia was in her forties, and held various middle management roles (including Māori Achievement facilitator), as well as teaching English and Japanese.

The student participants were Jacob and Briar. Jacob, aged 12 years and nine months when the study began, considered that he was the youngest student in the school. He identified himself as "Samoan." He was a student in Georgia's Year 9 Japanese class, and stated that "I really enjoy it and it's going well." He described his relationship with Georgia as "very good." Georgia herself described their relationship as "mostly good."

Briar was 15 years and four months old when the study began. She identified herself as "NZ Māori NZ European." Briar described herself as fairly proficient in conversation in French and Spanish, and having some words and phrases in Te Reo Mãori. She had had no prior contact with Georgia, although Georgia had been her year level dean. Briar was in Georgia's Year 11 English class at the time of the study, and stated that "I really enjoy it and it's going well." She described her relationship with Georgia as "very good." Georgia did not complete a baseline data template for Briar.

The parent participants were Pearl and Sandy. Pearl, Jacob's mother, a Pākehā woman in her forties, was a primary school teacher. Pearl stated that she had a fairly established, "very good" relationship with Georgia, in which the interaction had been "positive." Georgia also stated their relationship was "positive", as well as "collaborative." Sandy, Briar's mother, was a Pākehā woman in her thirties who worked part time in a clerical role. Sandy reported no prior interaction with Georgia.

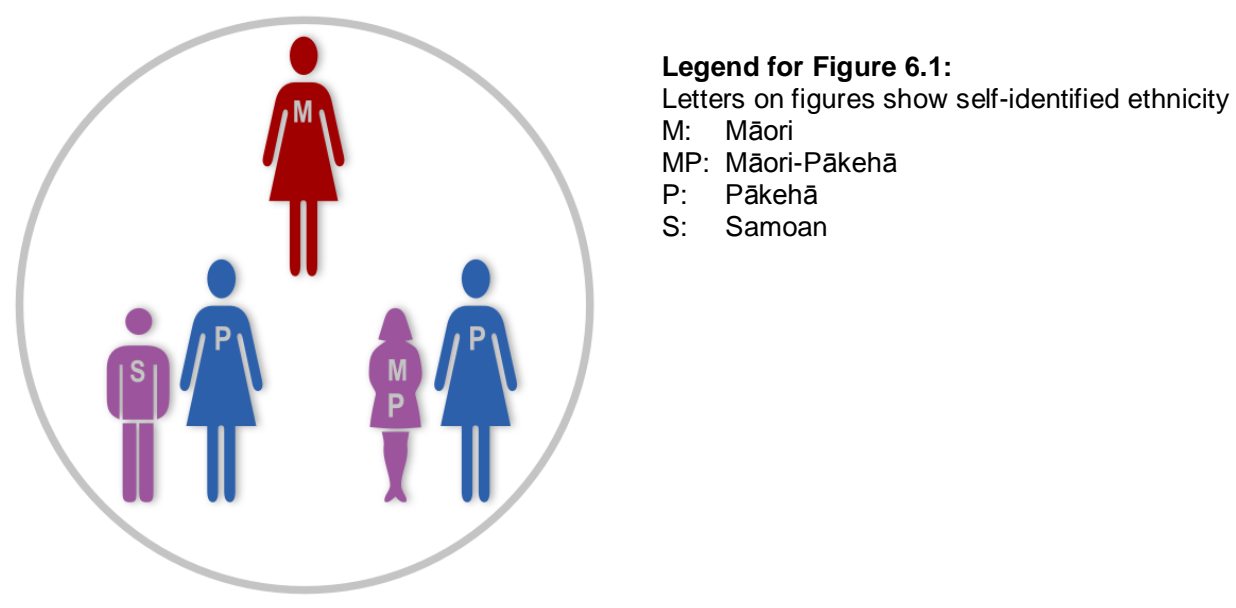

Figure 6.1: Team C participants 


\subsection{Synopsis of findings}

Briar, Sandy, and Pearl shared Georgia's hope that benefits from her new learning about students' FoK would include development of two types of knowledge: deeper knowledge of students as individuals and new knowledge about relating to and teaching students, which would improve the relevance of classroom learning, and increase motivation. Pearl theorized that:

if a teacher knows more about a student in a positive way, then they're more likely to have a better relationship... with more engaged learning and all the rest of it, 'cos the student feels valued, is likely to try harder, because there's a better connection there. (reflection)

Findings suggest that these outcomes were achieved by Team C's application of strategies.

Jacob hoped to improve his own focus on education through participation in the study (for example, by learning more about speeches and new vocabulary). He also wanted to be involved in an "adulty" (interview, line 339) project that could benefit the wider community.

Team members all valued reciprocal sharing of personal information; therefore, all team members gave a personal presentation on their FoK. Reciprocity was judged to be important to establish trust. Similarly, I was asked to present information on my own FoK, which I did at the final team hui.

The team dynamic was purposeful and collaborative, which provided encouragement and support for Georgia to experiment with ways to bring students' FoK into her classes. Also, collaboration between Georgia and I helped to refine ideas for classroom application. Georgia was inspired by her new learning, which highlighted the possibility of applying FoK in classroom learning to enhance motivation and relevance, without losing focus on learning goals from the curriculum and school qualifications.

\subsection{Evidence by theme}

\subsubsection{Preferred approaches for teacher to learn about students' FoK}

Team C members wanted to apply the FoK concept to classroom learning, and to ensure Jacob and Briar's FoK were highlighted for Georgia. It was also important to Georgia that she reciprocate by sharing personal information. 
Therefore, in Hui 2, Team C agreed to implement two strategies:

(1) Have a team get-together where everyone in the team brings some things that they love/ value/ consider an important part of who I am; (2) Georgia gets the classes to do a "Week in my life" activity. (Team C agreement 07-07-11.pdf)

In Hui 3, this team decided to add no further strategies. They were satisfied with their chosen strategies, and their implementation was ongoing, as the planned teaching activity had been conducted in one but not both classes.

\subsubsection{Enablers that supported valued outcomes in Team C}

\subsubsection{Articulate participants}

When selecting students to participate, Georgia decided to invite articulate students, so that expression of ideas would not be problematic, and fluent discussion could take place. To an extent her hopes were fulfilled, as evidenced by Briar's and Jacob's contributions in team hui. For example, Briar contributed elaborated comments about the class activity implemented in Year 11 English:

I thought it was good in class because everyone sort of talked and took a while to think about what they wrote. (Hui 4, lines 142-143)

However, Briar's verbal contributions were relatively infrequent, as shown in Table 6.1. This surprised her mother Sandy, who remarked "I know she's usually wellspoken and things like that, I did actually find that she sort of holds herself back a little as well" (interview, lines 14-15). Although Briar was articulate, she stated "I tend to write a lot more than what I say... I can put my words better here" (Hui 4, line 840844). Thus, she appreciated written reflections before discussion in team hui, so she could organize her thoughts, and this practice helped her express her ideas. Briar reported it took some time to get used to being in a team with her teacher and mother, which may explain the increasing frequency of her contributions over time.

Jacob contributed frequently to discussion about strategies in Hui 2. When I asked if someone would like to start by making a suggestion, Jacob immediately said:

Students bring items and photos that symbolize things that have happened or important things in their life. That's just a combination of (two strategies on the sheet provided). (Hui 2, lines 25-26)

He also volunteered ideas about effective teacher practice:

It's a good idea, a good suggestion. It's a good idea to try and get to know your students, especially me and Briar... and I think it will be a good opportunity to get to know the students, what their expectations would like to be, you know, and see what they would like to have in the future, try and push them up to where they want to be, and if they want to go down, just push them up a little bit more. (Hui 2, lines 205-216) 
This could be a good idea to the project (sic), because like, if you do baking and bring muffins, you could tell them, and if I said l'd made them it would be like funds of knowledge, like you know how to make them, and you know, you're telling people that you know how to make muffins at that age. (Hui 2, lines 449-452)

As revealed in Table 6.1, in Hui 2 when Jacob's mother (Pearl) was absent, he contributed ideas relatively frequently; however, when she was present his contributions were reduced. In Hui 4, comments relating to Jacob's experience of the class activity were mainly made by Pearl. For instance, Pearl related information about time Jacob spent on the activity and his evident enthusiasm, and Jacob seemed to be content with Pearl's description.

Parents in Team $\mathrm{C}$ were also verbally articulate, although Sandy lacked confidence and "often talked about herself in negative ways 'I'm not that good, I'm not...' (Pearl, interview, lines 304-305). Both Pearl and Sandy contributed fully to discussion. Unlike her daughter, Sandy liked to develop her ideas in discussion, and she made frequent contributions. Pearl stated "she was actually a brilliant communicator, she had a lot to say, she was very logical and she was very clear... and the interesting way that she would look at things l'd go yeah, I never thought of it like that" (interview, lines 309-311).

Table 6.1 shows the frequency of participants' contributions in team hui.

Table 6.1: Contributions of Team $\mathrm{C}$ participants in team hui

\begin{tabular}{lcccccc}
\hline & Jacob & Briar & Sandy & Pearl & Georgia & Linda \\
\hline Hui 2: number of items & 28 & 14 & 40 & (absent) & 39 & 49 \\
Hui 2: percentage of items & $16.5 \%$ & $8.2 \%$ & $23.5 \%$ & (absent) & $23.0 \%$ & $28.8 \%$ \\
Hui 3: number of items & 25 & 29 & 60 & 64 & 66 & 93 \\
Hui 3: percentage of items & $7.4 \%$ & $8.6 \%$ & $17.8 \%$ & $19.0 \%$ & $19.6 \%$ & $27.6 \%$ \\
Hui 4: number of items & 39 & 45 & 151 & 147 & 180 & 246 \\
Hui 4: percentage of items & $4.8 \%$ & $5.6 \%$ & $18.7 \%$ & $18.2 \%$ & $22.3 \%$ & $30.5 \%$ \\
\hline
\end{tabular}

Although the number of contributions by participants was different, Sandy, Briar and Jacob agreed opportunities to contribute had been equitable. In her Hui 4 reflection, Briar wrote:

I believe everyone in the team had an equal opportunity to express their ideas.
I believe the team was open to new ideas and perspectives. Friendly, easy-
going atmosphere. It was serious but you felt you could speak your mind to
get your point across.

However, Georgia and Pearl were unsure. Georgia worried that perhaps the "adult dominated/ guided conversation/ decision-making process" (Hui 4 reflection) may have got in the way. Pearl wondered if she should have held back more, to allow others' voices to come through more strongly. 


\subsubsection{Brainstorming together}

Collective brainstorming of possible ways to apply FoK to classroom practice and potential benefits helped the team express their identity as a collaborative research team, develop ideas, evaluate implemented strategies, and consider connections with other aspects of school life.

I observed that conversation between all participants in the team was open and fluid, and everyone had something to say. Briar identified the "best thing about what our team did" as "the group discussions. Everyone being involved and open" (Hui 3 reflection). For example, team members collaboratively developed ideas about strategies in Hui 2:

Jacob: $\quad$ Oh I also like students writing about their life experiences.

Briar: $\quad$ Oh yeah l've got that too.

Linda: $\quad$ Sounds like we've got a lot of agreement, so we have to hone in on which way we want to go. l'm thinking these are three ways you could tackle the same kind of aim.

Sandy: $\quad$ But you could do a week in the life in Japanese too, couldn't you? I know in Year 9, you'd just be learning how to write it, but they might be able to write some of their words in Japanese as well.

Briar: $\quad$ and compare it to...

Georgia: Yeah, and like school life. Yes, because life for Japanese students is so much different...

Linda: $\quad$ So you're thinking... so Sandy's saying that an idea could be a class activity?

Sandy: $\quad$ They could be writing about it, and writing about it in Japanese as well. 'Cos they'd be learning how to write their Japanese letters...

Georgia: Also their times and schedules and things, like a timetable, which we're getting into.

Sandy: $\quad$ That would be a good way to start learning it. (Hui 2, lines 85-115)

In this conversational thread from Hui 3, team members highlight the importance of mutual sharing, including sharing from me:

Georgia: Yeah, 'cos sometimes when the teacher gives a little bit of them in the classroom, it sort of opens the door.

Linda: $\quad$ More reciprocal... do you think it would have been different for you... would you have felt differently if you had simply been asked to share something about yourself, and Georgia hadn't done the same?

Briar: Yeah.

Sandy: $\quad$ Yeah, I think everyone should show.

Pearl: $\quad$ Otherwise it's too one-sided, and we're going yeah, we're telling you, so...

Sandy: $\quad$ Like we said, you know, when we said about you showing us.

Linda: $\quad$ Yeah, about me, 'cos here I am listening! 


\begin{abstract}
Sandy: It's a team effort, yeah.
Linda: $\quad$ Yes. And I do understand your reasoning, and I will do that.

Sandy: $\quad$ Just like when I was a kid, you've got your teachers and coaches of sports teams and that, wanting you to do something, but I think, well, if you're not prepared to do it, why should we?

Linda: $\quad Y e a h$, absolutely. How do you feel about that Jacob? That whole question about would it have been different if Georgia hadn't been sharing, if it had just been you guys sharing?

Jacob: $\quad Y$ Yeah, I think it would have been real different. Just sharing about yourself to somebody, and then they... if they then wouldn't tell you something about them, you wouldn't kind of trust them.

Sandy: $\quad$ You wouldn't feel as comfortable would you, 'cos it would be less personal. (Hui 3, lines 204-239)
\end{abstract}

Although all team members contributed to suggestions relating to strategy negotiation and evaluation, parents were very active in developing ideas. Georgia stated "I was especially impressed with parental input!" (Hui 4 reflection) and added that participants' "willingness to challenge and suggest" was helpful. Both parents drew on aspects of their identity to inform their contributions. Sandy drew on her prior experience as a school student to imagine students' possible perspective, in response to various activities. For example, when considering a possible strategy, she reflected:

If you did something like this, the students would think it's about me, it's not all about the stuff that you're just talking about every day and some of them are switching off to. Instead of them listening to a teacher just rambling on, something they're not interested in, most people would probably try and be involved in talking about a week in their life. (Hui 2, lines 277-279)

Pearl brought various aspects of her identity to participation, including mother, teacher, and wife. As a mother, she supported her son (see discussion of inclusive team interactions). Also she discussed her role as Jacob's mother to protect him in dealings with teachers:

That negative barrier's broken down immediately, and you're more likely to trust them if you know them - like, if they said this hasn't been going so well, you can go okay, what should we do, how can we deal with this - not just think instantly, this teacher's out to get my child... you can (have confidence) that the teacher's (acting) in the best interests of your child. I know that they are supposed to be, but feeling that they are, it's a whole different ball game. (Hui 3, lines 493-503)

As a teacher, Pearl suggested pedagogical ideas, and was aware of teachers' issues, such as workload. For example, she inspired Georgia to consider a variation of the Year 9 Japanese class activity in which she shared aspects of herself:

Georgia: $\quad$ Alright, l'll do that too. Good.

Linda: $\quad$ Alright, so it sounds like that's a bit of a next step. Not so much for the team, but for you. 
Pearl: $\quad$ Sorry mate.

Georgia: $\quad$ All good. (Hui 3, lines 750-767)

Pearl reflected on implications of team findings for her own teaching practice. She took notes of pedagogical ideas that emerged in discussion, stating "I was thinking so how could I thread this through... with my students? You know, how could I do it through reading and writing and topic?... How could I add this?" (Hui 4, lines 1216-1218).

Pearl also made connections between learning about students' FoK and her marriage. She reflected that it would be helpful to incorporate activities that drew out students' FoK throughout the school year, because:

I suppose like any marriage, where you know like 16 years down the track, I'm still learning things about my husband. It would be the same doing this, you'd learn different aspects of a person. (Hui 4, lines 1183-1185)

Pearl's marriage influenced her thinking about how family members and family dynamics contribute to individuals' FoK. For instance, she was conscious of the different values she and her husband were brought up with, which in their marriage has highlighted:

the whole process of choosing between two worlds of - family that pretty much still says you know this is the way to do things and you've got to listen to your mum and l'm always your mum - and negotiating a world where his wife says well actually... you've got to make up your own mind and have some own choices and you know I'm not going to always make the decisions for you, you've got to make some for yourself and we've got to make some together. (interview, lines 419-425)

Collaborative brainstorming in Team $\mathrm{C}$ was coherent within the overall dynamic of team interaction, which is best characterized as that of a collaborative research team. Georgia positioned herself as a learner, interacting with others to develop professional knowledge. Thus her interactions with others in the team were largely related to the team's function: listening and considering ideas, fulfilling her role according to the team agreement, and reflecting on classroom activities and impacts, with the help of data. Table 6.2 presents an overview of ways Georgia enacted her role.

Pearl was also focused on identifying data to verify perceived outcomes, as shown by the following examples:

So how would you measure that? (Hui 3, line 735)

I know that my son was more engaged. He came and talked about it, he did in his own time he was doing it in lunchtimes and I know that he had Simon Hodges at one time with him at the computer doing it. (Hui 4, lines 1137-1139)

I have a question... was it a high percentage of people that completed these types of work than what would normally be handed in? (Hui 4, lines 1317-1320) 
Table 6.2: Ways that Georgia positioned herself as a learner

Ways that Georgia positioned herself as a

Examples learner

Openness to suggestions "I'm quite happy to do whatever anyone wants me to do" (Hui 2, lines 202-203);

Listening and responding positively to parents' critique of pastoral care by form teachers (Hui 4);

Pearl: I was just wondering, did you share anything with them, like before you, as you led into this?

Georgia: No.

Pearl: $\quad$ Did you like go and say Oh no l'm thinking about this, and

Georgia: Yeah and absolutely maybe I could have... I mean I could have said well actually when I was younger...

Sandy: And some of them might not be that concerned about sharing it.

Georgia: Yeah.

Sandy: You know like, you could go back and say... would anyone be happy for me to share their...?

Georgia: That's right. (Hui 4, lines 207-230)

"So I could do that actually, I will do that, l'll write that down." (Hui 4, line 250)

Asking for or showing appreciation of help to develop strategy ideas

Expressions of doubt or uncertainty

"I wouldn't have even thought about it being a classroom thing until you said that." (Hui 2, line 167)

"Or do you think, things like, if I did bring in a pinboard?' (Hui 2, line 294)

Georgia: But maybe I needed to think of questions that were a bit less...?

Jacob: Personal?

Georgia: Yeah, a bit more generic, or maybe more doing it in groups and giving them a situation, more like what would you do... maybe I could have started them off more gently, I don't know." (Hui 4, lines 187-196)

Collecting data

"Jacob's done a family tree, and he's put in the names of the people, so he's put in you guys." (Hui 3, lines 31-32).

"I brought in some examples of what we were doing with the Year 9s. Sorry Jacob, I've got other students' (work) as well just to show he's not the only one that's actually doing the project." (Hui 4, lines 19-20)

Request to collaborate Organizing and conducting a meeting with me to collaboratively develop class activity ideas.

Identification of next learning steps

"For me... for my planning... there should be like a checkbox for this... Is what l'm doing going to show the students are valued? Is what l'm doing going to show how this relates?... within my planning 'cos I do, I have my own planning sheets and... well perhaps I need a box like this that I can say yes, and how l'm going to do it." (Hui 4, lines 998-1004).

I was also involved in collaborative brainstorming when Georgia sought my help and we collaborated to develop a writing activity for her Year 11 English class. Georgia persevered with the new challenge and developed a number of ideas before our meeting. She stated, “when I thought I didn't have any (ideas), I sat back and thought, try a bit harder" (meeting, 3m.10s-3m.17s). 
Georgia initially found it difficult to imagine how she might incorporate an activity that drew on students' FoK that was a relevant part of their study of Baz Luhrmann's movie, Romeo and Juliet. Because the Year 11 class was enrolled for their NCEA qualification, it was important that all classwork related to this goal. She asked to meet with me so we could reflect together. We developed the idea of getting students to write about the characteristics of boyfriends or girlfriends who their parents would find totally objectionable, therefore possibly causing a family feud. The following extract from my meeting with Georgia illustrates our interaction. Georgia had described her idea for a Day in My Life activity:

Linda: $\quad$ So you're saying that in this one, they could talk about their own families?

Georgia: In Japanese, and bring in photos of them, so that's quite good.

Linda: $\quad$ So would you direct them in any way? I mean, what sort of?

Georgia: Well, I don't, it's very limited, the Japanese they have, so it would be, this is my mother, her name is... she is... so and so years old that's all it would be. However, if I could tie it with countries, 'cos we're introducing nationalities, then we could say this is my mother, she's from wherever, so that might be a bit better, at the moment they just know this, and I'm going to be starting on this soon.

Linda: $\quad$ So l'm just thinking, 'cos you've got a range of themes coming up, what about the idea of consciously building something?

Georgia: Yeah? Like?

Linda: It's just a thought, you know how you said at this stage they can say, this is my mum, her name is Melissa, or whatever, and things like that, and then you're saying that later on maybe they could add

Georgia: Yeah, now l'm thinking that either maybe by the end they could produce a booklet that ties in to who they are, and the relationship with their families, and so we could do them all, and somehow it end up being a booklet, or a poster.

Linda: $\quad$ I just had an idea, you're talking about authentic. What about if that end product was to send to a school in Japan? (sic)

Georgia: Yes!

Linda: $\quad$ So that they can learn more about New Zealanders.

Georgia: Absolutely!

Linda: $\quad$ So that all this work that you're doing, they end up publishing.

Georgia: $\quad$ Yes that's great.

Linda: $\quad$ To help other people learn about who we are as New Zealanders.

Georgia: And also maybe... Yes that's great, 'cos I can get some contacts of a school that we could send them to, and maybe start a kind of, what's it called, pen... kind of conversation, dialogue, even if we could hook on via the internet to some 
kind of... but that's good! End product, so there's actually something, for them.

Linda: What do you think?

Georgia: That's great! Say "hi, I've learnt about your life, your life's really really busy, and this is what we do here. Great! This is who I am, that's very good. Like a portfolio of life in New Zealand, but my life, not just generalized, I'm a bit different, and my family, but that's what it would be.

Linda: $\quad Y e s$, not generalized information, but this is me and my family, this is what we do. (3m.30s-6m.55s)

Georgia valued the "good ideas" (Hui 4, line 259) from collaborative brainstorms in team hui. For instance, in Hui 4 Sandy and Pearl contributed ideas for possible refinements to the writing activity. Georgia wondered how she could adapt the activity to not only draw out their FoK, but also enable students to learn about each other. Options she considered were setting less personal questions and undertaking the activity in groups. Pearl asked if she had shared her own personal reflections on the writing topic beforehand - this question led to discussion between Sandy, Pearl and Georgia about how her personal sharing might result in students' greater willingness to openly share answers with each other. Also, this suggestion stimulated Georgia's reflection about how this might enhance her connection with students:

I could say I was reading your stuff and it was quite funny 'cos actually when I was younger I was a bit naughty, or these were some things I did... it doesn't matter how old you are, we were all teenagers once. (Hui 4, lines 239-246)

As mentioned earlier, Pearl also encouraged Georgia to consider incorporating activities that drew on students' FoK in different ways throughout the school year, and this idea was developed between the parents and teacher.

\subsubsection{Inclusive team interactions}

Team $\mathrm{C}$ achieved an inclusive environment with the following actions: courtesy and respect for each other; support for the youngest member, Jacob; sociability towards each other; and consideration of different perspectives in planning. Also my provision of opportunities for expression of ideas in writing and discussion was also experienced as inclusive by participants.

The team operated in a way that was respectful to all members, supporting the expression of ideas by all. Pearl stated that the team members were "good and polite communicators" (interview, line 313) who worked through disagreements in a way that "was constructive rather than destructive" (interview, line 320). She considered that "in this (team) everyone did value and respect each other's opinions immensely; it was really lovely" (interview, lines 333-334). 
The youngest team member, Jacob, benefited from the support of others in team hui, which was provided with patience, respect, and tolerance. For example, occasionally Jacob sought clarification:

Linda: $\quad$ For this one here, we might set a more vague timeframe.

Jacob: Does vague mean strict, or like whenever?

Sandy: $\quad$ No, not strict.

Linda: $\quad$ Yeah, like Sandy said, not so precise.

Sandy: $\quad$ Like probably in a few weeks. (Hui 2, lines 413-421)

Similarly, when the team agreement was circulated to be signed, Jacob asked about how to do his signature, later commenting "I'm proud of my signature, it's so professional" (Hui 2, line 507).

Jacob's mother Pearl supported him. After attending Hui 2 without Pearl, he summarized the meeting for her; however, later she realized that "it was quite different in his mind" (interview, line 511). From that point she adopted the practice of "checking in with him, making sure that he was on track and how is he finding it." (interview, line 513). Jacob reflected that in hui he would be "real adulty" (interview, line 339), and then sometimes "go into the childhood again" (interview, line 340). For example, in Hui 4, Jacob's attention wandered from the discussion topic:

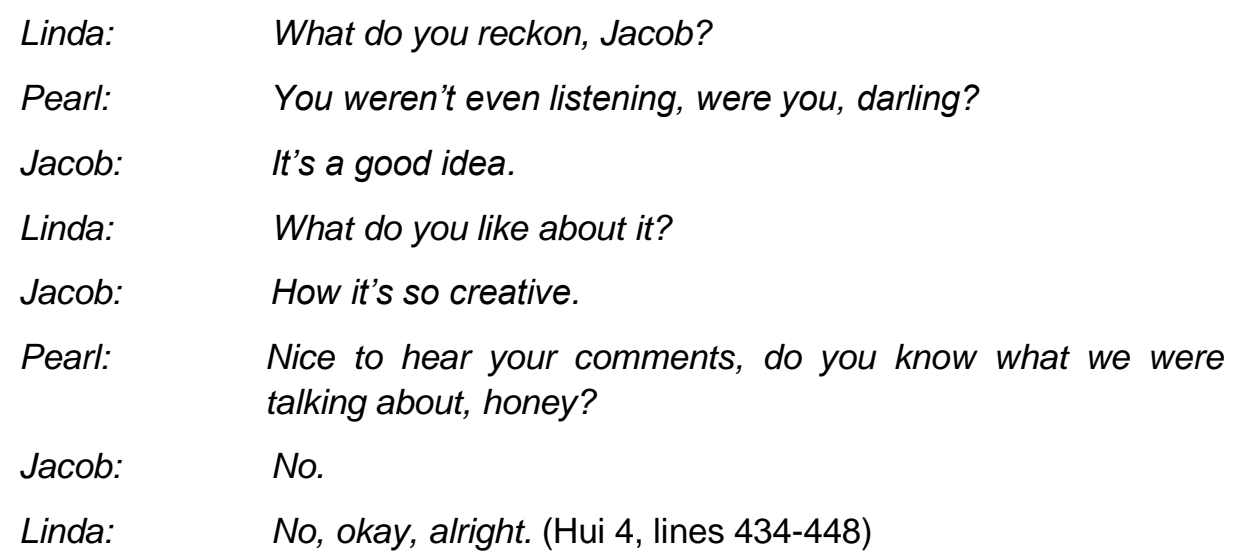

Therefore, I would argue that Jacob's desire to be involved in a serious project and willingness to ask questions when confused, combined with his team-mates' patient and tolerant response to his questions, enhanced the inclusive nature of the team interaction.

Sandy's contributions enhanced the sense of inclusivity in Team C. I noted in my journal that Sandy had strong socializing skills, which enhanced team bonding. For example, in Hui 4, when Georgia shared her daughter's recent dental appointment, she said, "Tell her she was awesome, an awesome girl" (line 742). Sandy's 
inclusivity extended to me; for instance, when my elbow was injured she enquired about its progress.

Sandy's inclusivity created a challenge for me in Hui 2 , when she caught me by surprise by suggesting that I also participate in the personal presentations:

$\begin{array}{ll}\text { Sandy: } & \text { And we'd like to see yours as well. } \\ \text { Linda: } & \text { Oh! But l'm the least important person! } \\ \text { Georgia: } & \text { Oh! } \\ \text { Sandy: } & \text { No, you're not! } \\ \text { Linda: } & \begin{array}{l}\text { How about if I say l'll do that at the end of project? 'Cos l'm } \\ \text { not trying to be secretive or anything, but I don't want to } \\ \text { distract attention from, you know, it's your relationship that this } \\ \text { is about, but I'm not trying to hide. }\end{array}\end{array}$

Sandy: $\quad$ That's fine, don't worry. (lines 482-494)

Inclusivity also extended to organization of team events, as shown by these comments related to setting a date for Hui 4 . Contribution of comments by a number of team members demonstrated care towards each other. At that stage of the school year, Briar's NCEA exams were imminent:

Georgia: I would like Briar to say when is best for her, since she's the difficult one. (line 823)

Pearl: $\quad$ How do you best study? Do you best study in the mornings or the afternoons? So if you best study in the mornings, we can make it for the afternoon, something like that. (lines 867-868)

Sandy: $\quad$ (To Jacob) Would you rather in the holidays? Is a time in the holidays alright with youse, or not? (sic)

Jacob: $\quad$ Oh, I don't mind, l'm not as busy as I used to be now. (lines 851-853)

Provision of both individual written brainstorming and group discussion in team hui also enhanced inclusiveness. Both Georgia and Briar stated their preference for some opportunity to consider thoughts in writing before discussion. Also, this was apparent to me through reading participants' body language. Therefore, time was routinely devoted to written activities early in team hui, as a result of my understanding that this was valued. I also stated to participants that they could choose how much effort to give to written activities. Although some members (such as Sandy) recorded very few ideas in writing, she tolerated provision of time for writing knowing that her daughter wished for it. Inclusion of both types of activities allowed opportunities to participate in a way that suited different individuals. These strategies were used in Hui 2 (regarding strategy likes and dislikes), and Hui 3 and 4 (for comments about implemented strategies). 


\subsubsection{Choice of strategies}

Personal presentations by all team members in the team get-together allowed everyone to learn about each other, and gave Georgia insight about the students through their own presentations as well as their mothers'. Pearl considered that the latter feature was beneficial because "parents are pivotal... what we value our kids will nine times out of ten value that same thing too" (interview, lines 380-381). She felt that this was particularly the case for younger students. Georgia agreed, stating:

It shows the importance of how family shapes the child. When we teach the student, we must be mindful that the student is more than who she/he presents herself to me in the classroom. (Hui 3 reflection)

Sandy believed that the format of the personal presentation meant "Georgia potentially could have learnt quite a bit about the students" (interview, lines 413414). However, Georgia felt "frustration that l've had only scratched the surface" (Hui 3, written reflection), because of constraints of timing and the formal structure of strategies used to learn about FoK.

Pearl considered that the team get-together worked well because the team comprised "articulate people" (interview, line 625). Therefore, speaking about oneself related to team members' strength. She stated "Jacob and myself, we just whipped it up pretty quickly" (interview, lines 626-627). In his final interview, Jacob also reflected that confidence with verbal articulation influenced a teacher's ability to learn about students' and parents' FoK. He commented "some people are kind of shy and don't want to like talk about personal things so much. So the people that really do like to talk... would be the stronger team" (lines 215-216).

The class activities implemented in Jacob's Year 9 Japanese class and Briar's Year 11 English class also supported the achievement of valued outcomes. The activities are described here. Also, data are presented which show that the class activities met their purpose of allowing learning about students' FoK. (Section 6.3.4.3 includes discussion of impacts arising from their application.)

In the Year 9 Japanese class, a portfolio activity was implemented to draw out students' FoK. For the purpose of the activity, Georgia made contact and collaborated with a high school teacher in Japan. All Georgia's Year 9 Japanese students were linked up with a penpal who was a high school student studying English in Japan. They were charged with the task of preparing a portfolio of information about their lives that could be sent to the Japanese students, to help them learn about what life is like for high school students in New Zealand. Replies from the Japanese students were expected before the end of the school year. 
Georgia's students also had the opportunity to present their portfolios to the class. Apart from its potential to draw out students' FoK, other notable features of the task were authenticity, due to the real use to be made of the student portfolios, and autonomy, due to the choices that students could make about the nature of information to include, and the presentation format. Selected presentation formats included powerpoint presentations, word documents, and brochures.

The Year 9 portfolio activity generated high interest for most of the Year 9 Japanese students (see Section 6.3.4.3). Jacob appreciated the opportunity to develop relationships with Japanese students that the activity offered. When he reflected on the team's two selected strategies, he commented:

I think that both of them would be a good idea because they're both like, getting to know each other, but like the powerpoint (prepared for the portfolio) was better because then people overseas would get to know you too and you would get to know them. (interview, lines 143-145)

He stated:

(The portfolio project) was like a good opportunity so I tried to put every single detail that I could put for myself in so that my project would be really up to it so that they would really know who I was. (interview, lines 503-505)

Georgia implemented a writing activity to draw out Year 11 English students' FoK while also contributing to their understanding of Romeo and Juliet. The activity required students to reflect about what type of boyfriend or girlfriend would really disappoint their family, possibly resulting in their rejection.

Georgia and Briar agreed that the Year 11 English writing activity successfully met the aim of drawing out students' FoK. Briar stated:

With that action we did in class I feel that it not only gave the teacher an idea of the students but it also gave the students a better idea of themselves. (Hui 4 , lines 1269-1270)

\subsubsection{Barriers to valued outcomes in Team C}

\subsubsection{Time and timing}

Team C participants agreed it would have been valuable for Georgia to begin learning about students' FoK and drawing on them in class activities earlier in the school year. Pearl expressed a shared view when she said earlier implementation would increase valued outcomes because "whatever happens now, it could have long-lasting benefits" (PFG3, line 453). Jacob also suggested an earlier start would allow team meetings to continue for longer. 
Two factors that contributed to time issues were the timing of team establishment and time pressures. The team met for the first time on 20 June 2011, and their preferred strategies were agreed on 11 July 2011, halfway through the school year. The team implemented their first strategy (a team get-together) within two weeks of agreement. However, slower progress was made with implementation of the second set of strategies, the class activities. Georgia did not manage to implement one of these activities before Hui 3 , as agreed. She stated "I wanted to do everything to ensure what I did in class was contextual, rather than superficial" (Hui 3, written reflection). Thus, although the team agreement referred to a "Week in the Life" activity, Georgia spent time considering an activity design that would fit more coherently into the two classes' learning programs, while also honouring the spirit of the team agreement. (Class activities implemented are described in Section 6.3.2.4.) She found it challenging to develop an activity for her Year 11 English class that met both these requirements. As discussed in Section 6.3.2.2, Georgia and I collaboratively designed the activity. It was implemented in class some time later, after Georgia had completed work that she considered high priority for the students' success in upcoming NCEA examinations.

However, there was agreement that learning about students' FoK would be valuable when the school year began:

Georgia: I would have liked this to have gotten underway earlier.

Sandy: $\quad$ Earlier in the year.

Georgia: $\quad$ At the beginning when I was supposed to be getting to know the students. (Hui 4, lines 967-971)

Pearl agreed, and also thought:

It should be bang smack at the beginning of the year and as a recurring thing, an ever-developing new thing because you present - it's like any relationship: you meet someone, you get to know them, you do the basics - this is who I am - and then as you get to know them more you delve a little deeper... It should be a continuous progression of knowing FoK that by the end it's like, I know this child, this student, inside out and I helped develop this student. (PFG3, lines 500-507)

Limited time between team hui added to time pressure experienced by team members, because it did not allow much time for strategy implementation. Therefore, "there wasn't quite enough time to get everything done" (Briar, Hui 4, lines 1441-1442). Both students agreed that time issues were the only constraint to valued outcomes affecting the team. 


\subsubsection{Valued outcomes achieved}

\subsubsection{New knowledge about others}

Knowledge of students' FoK gained from the two strategies was different, as shown in Table 6.3.

Table 6.3: Examples of students' FoK revealed by Team $\mathrm{C}$ strategies

\begin{tabular}{|c|c|c|c|}
\hline Student & $\begin{array}{c}\text { Examples of FoK } \\
\text { revealed by personal } \\
\text { presentations }\end{array}$ & $\begin{array}{l}\text { Examples of FoK } \\
\text { revealed by personal } \\
\text { portfolio activity }\end{array}$ & $\begin{array}{c}\text { Examples of FoK } \\
\text { revealed by Romeo } \\
\text { and Juliet writing } \\
\text { activity }\end{array}$ \\
\hline Briar & $\begin{array}{l}\text { Competitive rock'n'roll } \\
\text { dancing } \\
\text { Family } \\
\text { Pets }\end{array}$ & & $\begin{array}{l}\text { Family values } \\
\text { Personal values }\end{array}$ \\
\hline Jacob & $\begin{array}{l}\text { Sports skills (e.g., kilikiti, } \\
\text { basketball) } \\
\text { Extended family } \\
\text { Oral communication }\end{array}$ & $\begin{array}{l}\text { Where he's from } \\
\text { Family } \\
\text { Sports } \\
\text { Food }\end{array}$ & \\
\hline
\end{tabular}

The design of both activities Jacob engaged in potentially allowed sharing of diverse information. However, Jacob chose different aspects of himself to share in his personal portfolio for students in Japan and for his presentation to the team, although no data identify the reason. The two strategies Briar engaged in were quite different, as the personal presentation potentially allowed a wide range of FoK to be shown, whereas the class writing activity asked students to draw on a specific aspect of their FoK: values held by their family and by themselves as an individual.

Discussion in team hui explored the effectiveness of class activities in teaching Georgia about students' FoK. Georgia considered that before the study she knew the Year 11 students better than those in her Year 9 Japanese class; however, she developed new knowledge of them through the class activity:

It was quite uplifting really how much respect the kids have for their families, and you know what family means to them, which is - you think with teenagers they're like, wanna do everything myself and stuff the family, but it's very clear here that yes, I sometimes am naughty but if I brought home someone my family wouldn't approve of they might be disappointed, but I think that they'd say it's your life and you make - so it's that kind of thing, that they're just a bit older and a bit more independent, so it was really, really good. And one question I asked them was... what would disappoint your family? You know, if you brought someone home that...? Without fail pretty much all of them said bringing home someone who has got no goals in their life. (Hui 4, lines 81-90)

She also reported "learning a lot about the Year 9s" (Hui 4, line 1125), such as finding out about those who had been born outside New Zealand. 
Apart from the knowledge of Jacob and Briar gained by Georgia, all team members expressed enjoyment from learning more about each other: students, parents, and their teacher. Briar considered mutual learning was important. She reported "I see (Georgia) more as a person" (Hui 3, line 319), and considered that Georgia's understanding of her had moved from 3 or $4 / 10$ to 7 or $8 / 10$. She stated that to know her really well, Georgia would need to know her whole family. Briar felt that mutual learning achieved by herself and Georgia enhanced her sense of comfort and trust. Sandy considered that Briar would be inspired by learning about Georgia, because of connections between their goals and interests, such as international travel and history. Pearl considered that "learning about the teacher's FoK" (Hui 3, line 200) was of paramount importance. Jacob also agreed that mutual knowledge was important, and affected class interaction:

There's more respect that goes into your work and stuff you work on. You work extra harder, and stuff like that, and you just be more respectful than you normally are, instead of just doing the work. (Hui 3, lines 324-326)

The students also valued developing deeper knowledge of others. Jacob said that it was "pretty good because we were getting to know each other, getting to know about their cultures, and learning about the teacher as well" (SFG2, 06m.33s06m.45s). Briar appreciated getting to know her classmates better, through reading their writing, "understanding their personalities and their backgrounds as well, which helped" (SFG3, 00m.06s-00m.10s) ... "about how their families... influenced their decisions (which gave her) a deeper respect" (SFG3, 00m.59s-01m.10s) for them.

Georgia also identified the importance of students learning about each other in her concern that, through the class activities, "the downside of this for me is l've got the information and l've learnt, you know, but they haven't learnt more about each other" (Hui 4, lines 183-184).

Briar and Sandy reported that, since the study, Briar had sought Georgia's advice about future study, which she had not done previously. Briar stated she was more comfortable with Georgia now, both in and out of class, and "more confident asking her questions... for opinions with my subjects... and yeah I guess just talking to her as well (interview, lines 23-30). Briar considered that "in a perfect world, the teacher would sort of have like that same thing with the whole class, like the class will know something about the teacher, and the teacher would know something about the students as more than just like you know, their school work" (interview, lines 93-95).

Both parents gained new insights into their children. Pearl commented on her new knowledge of Jacob in Hui 3 and Hui 4. She stated: 
I didn't realize the kilikiti ${ }^{28}$ bat was so important to Jacob... I thought he was telling tamas ${ }^{29}$ at the time, but that was because (he was given the bat by his grandfather) when they were in Samoa together and I was back home. (Hui 4, lines 896-902)

Sandy also gained new insight into her daughter; she was surprised how reserved Briar was in discussion. Briar predicted "that her teacher will think, mm, she's quite different out of class" (Sandy, interview, lines 225-226), because she was "more outgoing in class" (line 224).

Sandy considered that learning about Georgia was more important for Briar than herself. Because Briar always did really well at school and had never had any problems, Sandy did not get involved with her school life. "I haven't got a clue who all her teachers are or anything like that because l've never had to come and have a talk with them" (interview, lines 249-250). Sandy now regarded her relationship with Georgia as established.

Pearl also valued getting to know Sandy and Briar:

I've heard lots about Briar through the years... but I didn't really know her...this has been such a lovely time to get to know her and see the amazing young woman that she is for myself. (Hui 4, lines 889-893)

Jacob and Pearl considered that learning more about students' FoK would improve the likelihood of teachers avoiding a reactive response when students behaved inappropriately. During the course of fieldwork Jacob was stood down from school after an incident in another class, and he reflected:

If they did get stood down, she would probably think of them as like a naughty person if she didn't know them better. (interview, lines 129-130).

\subsubsection{Applying FoK to classroom pedagogy}

Class activities, described earlier in Section 6.3.2.4, to draw out students' FoK were implemented in both Briar's and Jacob's class.

Initially Georgia was apprehensive about whether it was possible to incorporate an activity that drew on students' FoK in her senior class, due to the time pressures arising from their focus on work for NCEA qualifications, but this concern ultimately dissipated when she implemented the writing activity, noted its relevance to the topic being studied, and observed the students' focus and engagement. Briar confirmed in Hui 4 and her final interview that the activity enhanced her understanding of the movie, stating:

28 The game kilikiti is the Samoan version of cricket

${ }^{29}$ Lies 
You were thinking of it about yourself but you were sort of seeing how they would have answered the questions if they were asked them, yeah, it's like different perspectives. (Hui 4, lines 177-179)

Therefore, Georgia gained new positive experiences of developing relevant class activities which drew on students' FoK in ways that enhanced teaching and learning, inspiring new pedagogical ideas. Georgia reported that participation "makes me reflect on my teaching" (Hui 4, line 1020) and reported that "knowing now what I know I think it's extremely important, and it made my teaching just for those activities more tactical really" (interview, lines 303-304). Findings suggest that she reflected on overarching principles for her teaching practice and ideas for future strategies and learning activities. After implementing the writing activity to draw on the Year 11 English students' FoK, Georgia wondered:

... what it could lead to if l'd done it earlier in the year, so maybe it would be if I were teaching this again I wouldn't leave it, I would, it would be one of the first things I would teach rather than the last, so I could get to know them this way. (Hui 4, Lines 125-131)

Also, she stated her intention to utilize activities that drew on students' FoK "all the way through the year" (Hui 4, line 1180). Georgia agreed with Pearl's view that "in the wider world l've retrieved FoK from people l've met over the years, and it's been ongoing and... kind of layered, whereas this is a little less three dimensional" (interview, lines 65-68).

Georgia considered that her understanding of the importance of making connections with students was reinforced, because participation "focused me more" (Hui 4, line 1075-1076).

Team findings highlighted the importance of consciously seeking knowledge about students' FoK for Georgia. Prior to the study, Georgia considered that "once in a while (she said to a student) 'Remember you're good at this and I know that you do that,' but it wouldn't have been 'Okay, let's tap into that'” (interview, lines 308-309). As a result of the study she developed an appreciation of benefits arising from deliberately identifying and drawing on students' FoK, and had "more concrete (ideas)" (interview, line 313). For example, she considered “It shouldn't just be me as the teacher, that's exactly where we should be going is that whole shared knowledge. And certainly the kids would, not respect, but find value in their peers who are more like them than I am, having that knowledge and sharing it" (interview, lines 372-375). She had a range of ideas for other future class activities that could highlight students' FoK, and enhance reciprocal teaching and learning: students taking on a teaching role, sharing an experience with other students, and drawing on families as experts. 
Georgia stated her intention to adapt her lesson planning template, and focus on this aspect of planning until it became embedded in her practice:

Within the planning these things there should be like a check box like this you know: is what I'm doing going to show the students are valued? Is what I'm doing going to show how this relates? So it's really for me a checklist of good teacher practice. And within my planning... perhaps I need a box like this that I can say yes and how I'm going to do it...Are my students feeling valued? Are they more motivated to learn? (Hui 4, lines 1000-1008)

Therefore, Georgia's intended next steps were derived from valued outcomes achieved through implementation of strategies: drawing on students' FoK made them feel valued in the classroom, and enhanced their motivation and engagement.

\subsubsection{Increased student motivation and engagement}

Jacob, Pearl, and Georgia all agreed that the Year 9 Japanese portfolio activity generated benefits in students' engagement, both for Jacob and others in the class. In Hui 3, Georgia stated she was "excited about what the students are 'giving' (written reflection). She considered that the activity was:

... probably the best thing that we've done... this has kind of peaked their interest 'cos it's real and they're sending it to real kids. (Hui 4, lines 52-56)

The authentic nature of the portfolio activity provided students a genuine reason for their efforts, including those who were not planning to continue their study of Japanese. Georgia reported "they're actually all really into it" (Hui 3, line 356). Georgia had wondered whether the students might find the activity too difficult, due to their limited vocabulary at that stage of their study of Japanese language. However, she noted that, in order to complete the task accurately, students were motivated to ask questions, thus extending their vocabulary. Georgia observed that this activity generated higher levels of engagement and task completion than had been previously attained in the class. Pearl confirmed Jacob was "excited" (Hui 3, line 135) when Georgia first explained the activity to the class, and highly motivated to work on it. Pearl talked about Jacob's enjoyment of the activity in her interview and Hui 4. She stated that Jacob:

... was more engaged, he came and talked about it, he did it in his own time, he was doing it at lunchtimes, and I know that he had (another student) at one time with him at the computer doing it... he was definitely more focused. (Hui 4 , lines 1138-1148)

Jacob himself stated:

Showing yourself, and talking to other people, that you pretty much don't know... it's pretty exciting, talking to somebody and hoping that they'll reply... making new friends. (Hui 3, lines 348-354) 
He believed some students were attracted to the activity because they could prepare their portfolio on the computer, and "others were like really serious, like it's a good opportunity to put yourself out there" (interview, line 401). It seemed that Jacob belonged to the latter group, as he liked to make international friends, and planned to travel overseas. Sandy agreed that the establishment of Japanese penpals in the portfolio project was:

Awesome. I think they could just continue for years and years and they could make good friends with those kids over there, like the ones that are really passionate about doing Japanese more so. (interview, lines 446-448)

Nevertheless, there were four or five students in the class who found the portfolio activity too difficult, whom Georgia allowed to complete an alternative activity. However, Georgia reported that she was very pleased with students' interest and engagement, which were much higher than she anticipated.

When Georgia implemented the Year 9 Japanese portfolio activity, she did not share her intention to learn about the students' FoK with the class, and Jacob did not recognize it as the team's agreed strategy. Pearl regarded this as a clever type of "subterfuge" (interview, line 11), because she considered:

It was probably good that you didn't know it was a deliberate strategy otherwise he would be like, I don't know, maybe not Jacob but I would have been cynical in his position. (interview, lines 23-24)

Pearl stated that Jacob "realize(d)... the connection" (interview, line 15) between the portfolio activity he was enjoying and the study when the team met again for Hui 3.

The portfolio activity also provided useful formative assessment data for Georgia, as "by "doing (the task), they're also telling me what they know in Japanese" (Hui 3, line 364). Neither Pearl nor Jacob were sure about the impact of the portfolio activity on Jacob's achievement level in Japanese. Jacob stated "I think it's just like, just the relationship. I don't think like, like educational-wise it's done any harm or anything" (interview, lines 492-493).

In SFG3 and Hui 4 Briar reported that students were motivated to give a lot of thought to the writing activity, because they found it worthwhile. Georgia confirmed that all class members completed the task. Georgia also considered the activity was "controversial" (Hui 4, line 76) because the students thought the topic quite personal. She reported that "a lot of them asked whether anyone was going to read it apart from me and I said no. 'Cos obviously there's a lot of personal stuff in it" (Hui 4, lines 79-81). "They're like, do we have to share this? I said no, not with the class" (Hui 4, line 119). Some students chose not to name their work. Due to students' 
privacy concerns, Georgia worried that "the downside of this for me is I got the information and l've learnt, you know, but they haven't learnt more about each other" (Hui 4, lines 183-184). Nevertheless, Georgia had also anticipated that "they'd be bursting to (talk about their ideas) you know, and maybe if I was out of the room they might actually... be able to talk a bit more freely" (Hui 4, lines 150 154). Briar confirmed that students were curious about each other's answers and talked together about the writing topic afterwards.

Thus, findings highlight the success of class activities in enhancing students' enjoyment and engagement in class. I would argue that this was so because both provided students with a compelling motivation for their engagement, whether it be to make connections with other students living overseas, or to find out more about oneself.

\subsection{Conclusion}

Team C interacted as co-researchers. Within the team, Georgia took on the role of learner. With support from other team members and me, Georgia developed learning activities that drew on students' FoK that were congruent within the teaching and learning program. These activities achieved high levels of student engagement because students found them relevant and meaningful.

All members shared information about themselves in a personal presentation, including me. Reciprocity was judged to be important, for the sake of equity. Also, this allowed Georgia to learn about students from parents and the students themselves. Team members advised Georgia about ways she could share her FoK with classes to build connections and trust with students, and scaffold learning. All agreed that learning about and drawing on students' FoK should begin early in the school year, and be ongoing, to develop deep understanding.

Georgia developed a range of ideas for other future class activities to highlight students' FoK, and enhance reciprocal teaching and learning: students taking on a teaching role, sharing an experience with other students, and drawing on families as experts. 


\section{Chapter 7: Team D Findings}

\subsection{Introduction to Team D}

Team D's teacher was Paul, an Englishman in his forties who was a year level dean and taught English. Paul's teaching career spanned 12 years, four of them at KHS.

The student participants were two boys called Peter and Piri, good friends who were both in Paul's Year 9 English class. Peter was 13 years and six months old when the study began. He identified himself as "Māori Pākehā" and stated that English was his only language. The statement that he said best described him in the English class was "okay" and he categorized his relationship with Paul as "sometimes difficult." Piri also identified himself as "Māori/ Pākehā." Although English was his first language, he knew some Māori words and phrases. He was 13 years and four months old when the study began. Like Peter, the statement he said best described him in English class was "okay" and he categorized his relationship with Paul as "sometimes difficult." However, Paul evaluated their relationship as "mostly good."

The parent participant was Lorena, Piri's mother, a Māori woman in her thirties. Lorena was a cleaner. She reported that, when the study began, she did not have an established relationship with Paul, and stated their previous interaction consisted of information sharing from teacher to parent. Although Paul also perceived his relationship with Lorena as unestablished, he reported no previous interaction between them. Lorena withdrew before Hui 3. Paul also withdrew after Hui 3.

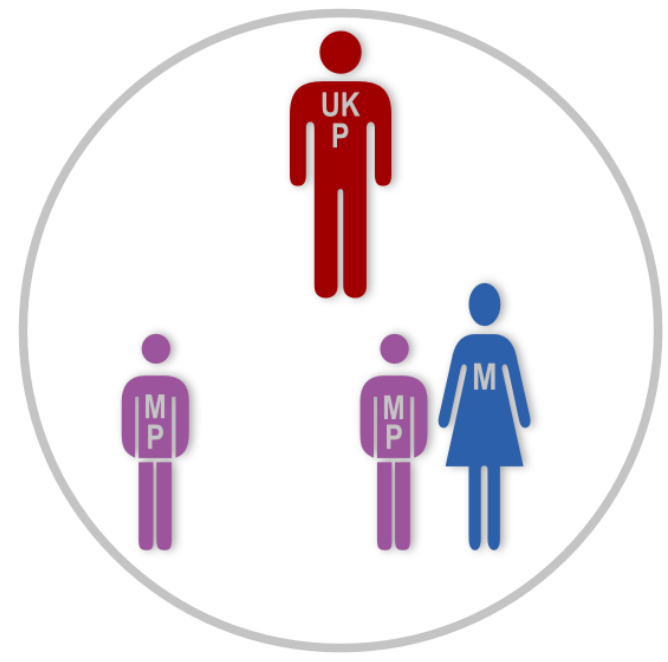

Start

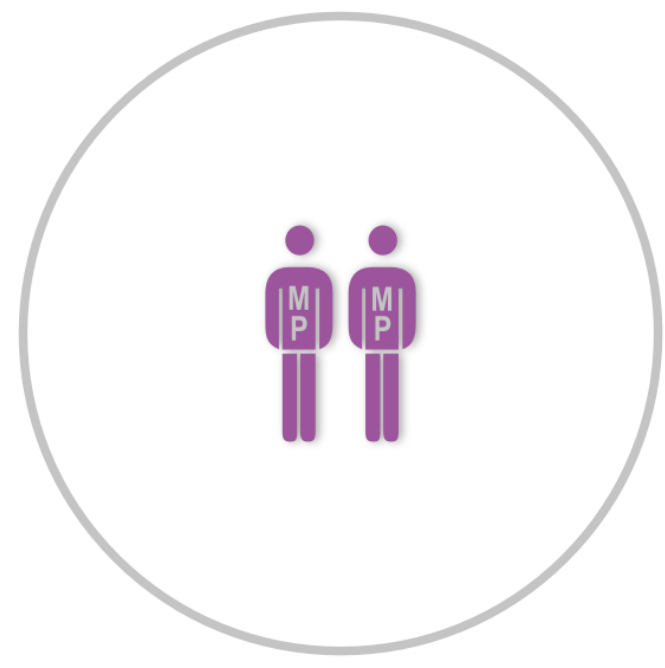

End

Figure 7.1: Team D participants at start and end of study

Legend for Figure 7.1:

Letters on figures show self-identified ethnicity

M: Māori

MP: Māori-Pākehā

UK P: UK Pākehā 


\subsection{Synopsis of findings}

Piri and Peter hoped when Paul learnt about their FoK, they would develop a closer bond with him, he would talk to them about their personal interests, and class lessons would relate to topics they were interested in, and draw on their FoK. Paul hoped that it would help him to develop fresh pedagogical tools.

In Hui 2, the team had difficulty agreeing on a strategy through which Paul would learn about Piri's and Peter's FoK. They decided by informal vote that the students would prepare videos to illustrate important elements of their lives and FoK. Implementation did not proceed smoothly, as the students missed a number of deadlines and attended one TM only after Paul sought them out in the school grounds, causing Paul to question the students' commitment. However, the boys reconfirmed their desire to participate, and eventually made a collaborative video and two short individual videos on the school site using my Macbook.

Paul felt he did not learn much from Piri's and Peter's videos, but in Hui 3 offered to reciprocate by making a video about himself. He also agreed at this time that the students could teach the class something, which Piri had suggested in Hui 2. Paul wanted the students to do this without support from him, feeling that provision of support would be too much extra work and would compromise the validity of the presentation as a representation of their FoK. However, shortly afterwards he withdrew, stating that he did not believe the students were committed, and that the benefits were not significant in relation to his effort. Paul allowed me to draw on data collected from his participation, and agreed that the boys' planned class teaching session could go ahead. The implementation of this strategy was also delayed, due to readiness issues. As a device to show their FoK, Piri and Peter viewed their teaching session as a failure; despite their considerable expertise in the general topic they chose, last minute changes to their plan and lack of access to equipment contributed to a lack of fluency and detail. Due to his withdrawal, Paul's own video did not proceed.

Both Peter and Piri stated their relationship with Paul improved over the period of the study, because he now understood that they liked to joke around, gave them more help, and offered them easier work. Paul stated he learned very little about the boys' FoK - no more than he could have learned from quick chats. He also considered that the boys' home life challenges were important for him to learn about, so he could offer them empathy. 


\subsection{Evidence by theme}

\subsubsection{Preferred approaches for teacher to learn about students' FoK}

Team $D$ determined strategies for implementation by informal vote when consensus could not be reached, as described in Section 7.3.2.1. The participants' preferred strategies were diverse and apparently irreconcilable.

Piri had well-developed ideas about teaching the class, to demonstrate FoK. Peter favoured surveys or an interview, because they would not involve too much work or too much writing. Paul preferred that the boys make a video that would provide a basis for discussion of both the boys' FoK and critical analysis of the video design.

Their team agreement records that in Hui 2 they agreed

Piri and Peter will each make a movie about themselves, using their own family cameras. They will share this with (Paul) and talk about it together, and he will have the opportunity to ask them more questions about things in the movie. (The school will try and help with equipment if necessary.) Linda and Lorena (Piri's mother) will also be there. (Team D agreement 22-06-11.pdf)

Also recorded in the document was the agreed date and time, two weeks hence, when the videos would be shared.

When the team met for Hui 3, Paul suggested it was appropriate for him to share something of himself, to reciprocate the boys sharing about themselves in their videos. In Hui 3, Piri was still keen for his preferred strategy to be reconsidered. On this occasion the teacher agreed.

At Hui 3, team members agreed that:

Paul will make a video to share some things about himself with the boys. This will be done by Monday 26 Sept. The boys will view this separately, with Linda, on 26 Sept. The boys will have the opportunity to teach the class something, later this term. (Team D agreement Hui 3.pdf)

\subsubsection{Barriers to valued outcomes in Team D}

In contrast to outcomes arising in other teams, valued outcomes for Team D participants were minimal, creating a negative case. Thus, in order to extract as much learning as possible from this team's experience, my analysis of data for Team $D$ focused heavily on building understanding of what went wrong.

Discussion of findings draws on more than twenty data sources collected over a five-month period, June - November 2011. Although Team D's teacher, Paul, withdrew from the study in September, he graciously allowed me to use data collected from his participation, and also consented to be interviewed in November 
2011. I am very grateful for these permissions, which I believe enhance my learning arising from Team D's experiences.

Although five themes related to barriers are discussed separately, links between them were apparent, illustrating the messiness and complexities between the multiple factors at play.

\subsubsection{Decision-making and power issues}

It quickly became apparent in the first team meeting (Hui 2) that Paul and Piri held different views about preferred strategies, which they each stood by. Paul put forward arguments relating to the merits of his suggestion that the boys make movies to demonstrate their regular activities and FoK. In response, Piri simply maintained his preference; he wanted to teach something to the class. A decision was grudgingly reached only with the pressure of time; when the one-hour hui was almost over, Piri stated "I'll just go with the video" (Team D Hui 2, line 470), seemingly indicating concession rather than willing agreement.

Although a democratic process was followed in Hui 2, two elements effectively reduced the students' status and power within the process. Firstly, when the hui began, Paul offered to speak first, and immediately stated his opposition to various options because he believed the students would not be comfortable with them. In so doing, it seemed his intention was to show understanding and sensitivity towards the students' perspective. However, his assumption that strategies that placed the spotlight on the boys would be anathema to them proved incorrect, and when Piri expressed his preference to teach the class something, Paul did not support the idea. Furthermore, in advocating an alternative strategy, Paul opposed Piri's preference. This apparent contradiction suggested that Paul had other reasons for not preferring the students to teach the class something. One possible reason became evident later in the hui, when Paul expressed uncertainty about whether it was allowable for him to implement Piri's idea within the IS program framework.

Paul's stated uncertainty about his autonomy as a classroom teacher created a dilemma about the decision-making process and possibilities. Peter expressed confusion about the range of allowable options, and his own status as a decisionmaker, asking 'Um, oh can we do the students' choices? Aren't we allowed to do that?" (Hui 2, line 422). Later, in Hui 3, Piri asked "Mister, are we still allowed to teach our class something?" (line 590), adopting a stance of seeking his teacher's permission, rather than engaging as an equal partner in discussion. 
By means of two actions in Hui 3, possibly unintentionally, Paul assumed the power position. Firstly, in acknowledgement of movies Piri and Peter had made for him to share their FoK, Paul offered to reciprocate. Framed as a show of appreciation for the boys' movies, the suggestion was presented as an offer, and agreed with very little discussion. However, its delivery as an offer effectively highlighted Paul's status as a person whose offer would be accepted as of right, unlike Piri, whose offer to teach the class was not agreed.

Secondly, when asked, Paul immediately agreed that the boys could teach the class: "You could teach them. I can't see why you couldn't teach them something that you know. They could do a Flying in Five ${ }^{30 "}$ (lines 593-4). In agreeing to Piri's preferred strategy, Paul's language suggested granting of permission. (Interestingly, this permission contradicted Paul's earlier expression of doubt in his ability to implement a new pedagogical initiative in the classroom.) Paul's immediate agreement to Piri's renewed suggestion (as a Flying in Five) seemed generous, given his earlier misgivings. However, because it was accepted without debate or discussion about the details, it effectively created a conditional agreement. It is unclear why Paul suggested the boys conduct their teaching as a Flying in Five. Due to the nature of a Flying in Five activity, the response effectively placed a very tight frame around the boys' teaching, in contrast to Piri's initial vision "where we can be the teacher for a day" (Hui 2, line 135). Therefore, the resulting agreement seemed to be a compromise reached simply through Paul granting permission, rather than by discussion by team members on an equal footing. The agreement arising from Paul's decision also generated a situation whereby the boys' presentation would be decontextualized - a one-off event before the lesson proper began. Despite their enthusiasm, implementation of the Flying in Five teaching session was not straightforward for the boys (see Section 7.4.2.2).

Looking back at Team D's Hui 2 and 3 gives me pause for reflection about my own facilitation of these team meetings. Retrospectively, I consider some of my actions may have backfired in their intent. For example, attempting to raise the profile of the students' voices, I often directed the question "What do you think?" to the students. I wonder whether the direction of this question to them actually placed more pressure on the boys: to provide the burden of argument for their ideas, to agree. However, during Hui 2 I also questioned Paul about how Piri's suggestion could work in the context of the class, and brainstormed with him how Piri's idea might look if it was implemented within the current unit of work.

\footnotetext{
${ }^{30}$ A Flying in Five is a short introductory activity usually scheduled at the beginning of class.
} 
Another aspect of my role in Hui 3 also took power away from the students. After the boys accepted Paul's offer to make a movie, Paul asked me to describe actions other teams had chosen to take, presumably to see if any excited Peter's or Piri's interest. As I listed teams' agreed strategies, the boys showed interest in actions taken by different participants whom they knew. Due to my inattention to time, this process took eight minutes, and as I drew the descriptions to a close, the bell called for the beginning of afternoon class. In the rushed final minutes, Piri again called for agreement to carry out his suggestion, and Paul readily agreed. Unfortunately, the timing limited discussion of details. At the time, the students and I felt this was problematic, but Paul was reluctant to spend more time on it:

$\begin{array}{ll}\text { Linda: } & \begin{array}{l}\text { Shall we get together, and have a bit more of a talk about } \\ \text { that? l'm thinking you might need a bit more of a talk about } \\ \text { that, just to kind of figure it out... }\end{array} \\ \text { Piri: } & \text { Yeah, we'll need to know what we'll teach. } \\ \ldots & \\ \text { Paul: } & \text { Do you need me there for that? } \\ \ldots & \\ \text { Piri: } & \text { Mister, you could tell us to teach something, and we could just } \\ & \text { teach the class. (Hui 3, lines 605-695) }\end{array}$

Ultimately, the presentation, which was intended to provide further learning about the boys' FoK, barely hinted at their knowledge of the chosen topic. Data gathered in my journal reflection following a preceding meeting, when the boys had brainstormed their ideas, and data collected in an interview after the presentation took place confirm that not only was their knowledge of both games detailed, but also the boys had clear ideas about connections with their academic learning, and ideas about how to effectively teach their peers. Thus, because the boys' FoK remained unexpressed, the presentation did not fulfill its purpose. I would argue that in this case, with additional discussion about details, support for preparation of the presentation may have improved the outcomes achieved.

Interestingly, despite my perceptions of power dilemmas in Team D's decisionmaking process, no team members reported similar perceptions or negative feelings. I would argue that the exercise of power within the team reflected the teacher-student roles and relationship as they played out in other settings, suggesting that the interaction may have been perceived as 'normal' by team members. Therefore, in this case, the lack of data from participants in relation to the theme of power could suggest how challenging it can be to shift from enactment of well-established roles towards more participatory models of interaction. But to what degree did the students' lack of enthusiasm about the strategy decided in Hui 2 
contribute to implementation problems? When actions are collaboratively agreed, a sense of shared ownership can assist smooth implementation. Therefore, questions remain in my mind about the degree to which these processes, however 'normal' for typical teacher-student interactions, tainted subsequent interactions and outcomes achieved.

\subsubsection{Implementation issues}

Team $D$ experienced delays throughout the project, as implementation deadlines arrived and went by with agreed actions not carried out.

The strategy agreed in Hui 2 was that the boys would each make a video to show the teacher their regular activities and FoK. A day before the boys were expected to deliver their movies to the teacher, Paul emailed me to express concern about the boys' commitment, because when he reminded them about it, they asked "What are we supposed to be doing? When?" The boys arrived empty-handed at the scheduled meeting, saying they had both forgotten about their agreement, but were still each keen to make a movie. I met with the boys again 19 days later, and once again they reconfirmed their interest in participation, and again asked for clarification about what they were doing. In an attempt to help them become more organized, we brainstormed possible ideas for video content, and I photocopied the brainstorm notes for the boys. I also tried to indirectly encourage Peter to consider an organizational strategy, as shown in the following exchange:

$\begin{array}{ll}\text { Peter: } & \text { Oh far, I've got heaps of things to remember for tonight. } \\ \text { Linda: } & \text { Okay, so how do you remember when you've got stuff to do? } \\ \text { Peter: } & \begin{array}{l}\text { Oh I just remember. Like I'm sitting on the couch and I'm } \\ \text { bored as, and l'll start thinking about what happened at } \\ \text { school, and then l'll remember. } \\ \text { Linda: }\end{array} \quad \begin{array}{l}\text { Gee, you're good, 'cos this is why l've got my diary - if I don't } \\ \text { write something down, I might forget. }\end{array} \\ \text { Peter: } & \begin{array}{l}\text { No, I won't forget. (Meeting with Team D students, 12-07-11, } \\ \text { lines 90-100) }\end{array}\end{array}$

The ongoing forgetfulness of the boys was frustrating for both Paul and me. Shortly after Hui 3, Paul decided to withdraw from the project, citing the boys' "lack of commitment" as one of his reasons. (Paul's withdrawal is discussed more fully in Section 7.3.2.3, relating to the theme of commitment issues.)

Problems concerned with lack of certainty about what they were doing also affected implementation of the boys' short collaborative teaching session decided at Hui 3. Planning difficulties arose from Peter's frequent absences from school combined with the boys' inability to contact each other outside school. The two boys had 
brainstormed their ideas, but Piri later realized that their plan was flawed; they wanted to teach the class about the Brumbies Challenge, a computer game which used mathematics skills, but this game could not be accessed within restricted account parameters at school. He knew about an equivalent game (BBC) that was accessible through students' school IT accounts, but could not discuss this issue, or the alternative option he had thought of, with his absent friend. Consequently, when the boys conducted their teaching session, it began with Piri briefing Peter about a change of topic. The last-minute change of plan contributed to an uncertain and uninformative presentation, which everyone agreed failed to achieve its aim of demonstrating the boys' FoK.

\subsubsection{Commitment issues}

The students' forgetfulness and apparent unreadiness to deliver actions or products according to team agreements were interpreted by Paul as indicative of a lack of commitment to the study. His feeling was compounded when one day the boys were absent from a lunchtime meeting, and, looking to remind them, he found them hiding. On this occasion Paul brought the boys to see me, stating that they had expressed the desire to withdraw. I asked them to clarify whether they did not want to be in the study at all, or whether they did not want to be at that specific meeting. Their reply to me was that they wanted to continue their participation, but had not wanted to attend a meeting in that lunchtime slot.

Paul remained adamant that the boys were simply participating because they wanted to please the adults, and could not express to me their wish to withdraw, due to my position of power and status as a university faculty member. This led me to deliberately recheck the boys' status as willing participants whenever it seemed appropriate, in order to try to minimize this possibility. For instance, in a meeting with the boys soon after Paul first expressed concern about their commitment, I gave them several opportunities to express their wish to withdraw, as well as firm guidance about what participation entailed:

Linda: I heard that you guys had said to Paul that you didn't have your video things ready, and first of all I wanted to check that you were cool about doing that? (lines 14-16)

Linda:

So where are we at with that then, are you going to do that? (line 28)

Linda:

So listen guys, I suppose whatever happens, whatever we decide, we really, we need to make it happen, so are you in or are you out? (lines 120-121) 
Linda: $\quad$ I want you to just think about that for a sec, 'cos if we're gonna meet, I want you to be ready, alright? (lines 143-144)

Linda: If you're gonna do it, you do it. If you say yes, then you do it. (line 148)

For all of these questions, the students' responses reaffirmed their commitment to participate, giving me a perspective that differed from Paul's, which in itself may have contributed to his sense of exasperation.

Paul's doubt about the boys' commitment remained, influencing, at times, his interpretation of their words and his behavior towards them. When Paul told Piri that he had not prepared his video (according to the team agreement), Piri responded "That's okay Mister, take all the time you need" (journal, 26-09-11, line 12), which to Paul reaffirmed his indifference and lack of commitment. Paul decided not to prepare the video that he had originally offered to make, because he did not want to reciprocate above and beyond his students' efforts.

Soon after Hui 3, Paul withdrew from the project, stating as his reason "I don't feel they were on board, as keen as I would have hoped" (journal, 26-09-11, line 30). He thought the boys would "feel relieved that they don't have to go through the process" (journal, 26-09-11, line 25). He restated his belief that the students were not committed to the study in an interview seven weeks later, due to their not being prepared for meetings and lack of progress on agreed actions. He wondered whether the participation of a mum in the group may have "grounded the project, by providing an extra person who could remind the boys about their commitment" (interview, 14-11-11, lines 16-17).

Paul's withdrawal was perceived by the boys as a type of abandonment:

Peter: $\quad$ He just let us down. Oh and...

Piri: $\quad$ Like we done this for him but he just, then he goes and...

Peter: $\quad$ And he couldn't do it.

Piri: $\quad$ Stops. (interview 2, 25-11-11, lines 455-461)

\subsubsection{The problem of silence}

In Team D, unspoken words and silences played a significant role in the way the team functioned, which may have had negative implications for outcomes achieved.

In Hui 2, Paul and Piri each took up an entrenched position about their preferred strategy; their respective statements or arguments were generally met by silence from the other. This occurred four times during Hui 2. Therefore dialogue (which 
may have helped the team work towards agreed strategies that were acceptable to all) was limited, and compromise did not occur.

Implementation of the students' collaborative presentation for the class was also marked by silence/ non-communication. The boys' Flying in Five teaching session was originally scheduled for 10 November 2011. It did not occur that day, due to Paul's judgement that the boys were not ready. On the day, Peter, early to class, said "Oh Mister, Piri's just getting changed after Phys Ed" (notes from audio reflection, 10-11-11, lines 7-8). When Piri arrived a few minutes later, Paul had begun teaching, and there was no communication between them at all about the planned Flying in Five. As an observer ${ }^{31}$, I found the situation confusing, and kept expecting Paul to call the boys up for their presentation. However, he conducted the whole lesson without referring to it at all. Afterwards I apologized to him for being present in a lesson which was ultimately unrelated to the study:

Linda: I'm sorry, I didn't mean to observe your lesson, I thought Piri and Peter's presentation was going to happen.

Paul: $\quad$ They weren't ready.

Linda: Weren't they?

Paul: Piri, Piri, were you ready?

Piri: $\quad$ Oh no. (notes from audio reflection, 10-11-11, lines 21-30)

My own further conversation with Piri suggested that matters were more complex than they appeared from the face value of these words.

Linda: Do you want to do this?

Piri: Oh yeah, yeah, yeah.

Linda: But we organized to be ready today.

Piri: $\quad$ Oh yeah but we would have done it.

Linda: But you told Mister you weren't ready.

Piri: Oh.

Linda: What would have happened if he'd called you up to do it?

Piri: $\quad$ Oh yeah we would of (sic) done it. (notes from audio reflection, 1011-11, lines 59-73)

This exchange raised several questions for me. Firstly it seemed apparent that Paul and Piri attached different meanings to the idea of readiness, with Paul giving it the meaning of willingness to perform the task, and Piri appearing to give it the meaning of preparedness for excellent performance of the task. Navigating my way through Piri's seemingly contradictory words, I believe that in saying he was not ready, he

${ }^{31}$ I was present to record the Flying in Five teaching session. 
was acknowledging that he could be more ready. In my journal, I also mused about the role of unspoken words:

I felt that there were a whole lot of unspoken rules in the situation: Piri seemingly dressed at his own pace after PE and then arrived. I suspect that he arrived too late for Paul - A Flying in Five is an introductory activity - need to arrive on time, but l'm not sure Piri realizes the importance of that for the activity. Carrying out the boys' presentation requires quite a lot of goodwill on the part of the teacher, or information - could have done it at the end of the Flying in Five, or nearer the end of the lesson, or at the end. I kept expecting something, but nothing happened. The other thing that's needed is they need to know everything that he needs for them e.g., if he requires them to be early, to be prompt, to confirm that they are ready - they need to know that. (notes from audio reflection, 10-11-11, lines 78-85)

Later, Paul confirmed that he "wanted more noise, some kind of indication from them that they were ready" (interview, 14-11-11, lines 84-85), highlighting the existence and role of a mismatch between the teacher's and students' ideas about appropriate behavior in that context, to achieve mutual understanding and agreement about carrying out the planned action. This incident raised Paul's level of frustration with the students, which I would argue reduced the possibility of positive outcomes from their eventual presentation. Together with the students' earlier behaviors of forgetting what to do, and missing deadlines, this reinforced Paul's focus on student deficits (see Section 7.3.2.5), rather than their resources and strengths.

The students' presentation was rescheduled, but its enactment a week later was also marked by problematic issues of silence. As described earlier, Piri's unilateral decision to change their teaching topic was unknown to Peter until the boys stepped up to the front of the class for the presentation. Piri had been unable to communicate with Peter in the preceding days due to his absence from school and their lack of access to phones. They had been in a class together earlier on the day of the presentation, but apparently did not discuss it; possibly there were no opportunities to do so. Consequently, Peter felt unable to contribute with any confidence. He said, "I felt kind of stink 'cos I was, I kept saying to Piri what, like keep talking, but I wasn't doing really nothing I was just sit... like hiding myself" (interview, 18-11-11, lines 259-260). Reflecting back, Piri stated "I shouldn't have done it... I should have asked Peter what one he wanted to do first and if he wanted to change I would have changed" (interview, 18-11-11, lines 268-269).

Peter and Piri were eager for Paul's approval, but he gave no verbal feedback or response to the presentation. Earlier, in response to the boys' movies, Paul's feedback consisted of telling them that he had gained some insights into their 
personalities, and that he noticed they enjoyed doing the video. Paul's silence after their presentation generated unanswered questions for the students:

Piri: $\quad$ Did he like it Miss?

Linda: You're not sure whether he liked it?

Piri: I didn't even...

Peter: Oh other teachers said it was good.

Piri: $\quad$ He hasn't even said anything to us. (interview, 18-11-11, lines 597-608)

The boys wondered about Paul's feelings:

Peter: He was just like at the back just folding his arms like trying to get the point of the game but he wasn't really getting it. (interview, 18-11-11, lines 559-560)

Piri: He didn't look very good 'cos he didn't have a smile, he was just looking at us like when are youse (sic) finishing 'cos I want to teach my class now. (interview, 18-11-11, lines 568-569)

Paul stated that he gained very little knowledge from the boys' movies, and the boys gave a poor representation of their FoK in their presentation to the class, adding, I believe, to his feelings of frustration and anger regarding the way that implementation of the strategies had played out. Earlier Paul showed that he valued reciprocity - giving back in a manner befitting to the other's gift. Therefore a possible interpretation is that Paul withheld feedback, feeling that it had not been earned. Nevertheless, the lack of positive feedback left the students' hopes for a closer bond with the teacher, and that the teacher would talk to them about their personal interests, unrealized.

7.3.2.5 Tensions between the conceptual basis of the study and Paul's beliefs about teaching

When the study began, Paul recorded his hope that involvement would "add some new 'armour' to my teaching pedagogy" (Questionnaire One for teachers, 13-06-11). Reflecting on what valued outcomes he hoped learning about the students' FoK might achieve, he wrote:

I want some new 'fresh' approaches to be available to me. I want a better understanding of pupils from New Zealand and how I can use their funds of knowledge to drive and inform my teaching. New experiences for me will hopefully keep me 'fresh' and 'keen' with my teaching. (Questionnaire One for teachers, 13-06-11)

Although Paul wanted to gain some fresh pedagogical ideas from learning about the students' FoK, a number of points of tension became evident between his beliefs and the principles that underpinned the study, thus adding conceptual barriers for 
him in undertaking the work. Findings are informed by data collected from Paul, as well as my thoughts about possible interpretations of his words and actions.

TENSIONS BETWEEN LEARNING ABOUt TWO StUdENTS' FOK AND PEDAGOGICAL DECISIONS TO BENEFIT THE WHOLE CLASS

Paul's ideas about what it meant to act professionally constrained his willingness to implement new pedagogical strategies in a class as a result of learning about just two students. He talked about this on a number of occasions, including several exchanges in TFG1. In this example, other teacher participants offered ideas about ways that he could create links between the students' FoK and their learning in his class:

Tom: I think that the content is the most interesting thing, that if you could use that content. I found when they were giving speeches, for example, that all those who gave religious speeches... there's a sense in which they really got into it... she was stronger and more confident and a better speaker as a result, and I think if it works as a platform then it works.

Paul: But are you talking about the content then, so the religious content?

Tom: Yeah, but a speech is quite a good form for our kids.

Linda: So what you're saying is when you're doing speech-making, encourage them to speak about those things about which they are passionate.

Tom: Yeah, absolutely.

Paul: But again, that in itself is contrived, contrived is the word, it's slightly contrived, especially as we're using speeches in English.

Lizzie: The whole religious thing as well, fits in nicely with your Diego Run. There's that whole element, they talk about the Earth Mother don't they, as being the Indian people's, so you could look at that, you could look at creation myths from the Bolivian side, you could look at how that ties in with Rangi and Papa ${ }^{32}$, and then also maybe bring in, 'cos the Catholicism's strong in South America as well, and sort of the Christian side of stuff, I don't know.

Tom: Gabriel's coming down to open the jail up, isn't he. That whole magical realism thing.

Paul: Don't get me wrong, I think you're absolutely right. You can find the links, it's the context as much as anything. I don't want to shove the text one way so I can bring in Peter's... (TFG1, 2h.39m.53s$2 \mathrm{~h} .43 \mathrm{~m} .06 \mathrm{~s})$

Therefore, although Paul acknowledged his colleagues' suggestions at a conceptual level, he did not consider it appropriate for him to implement any of the suggestions on the basis of one or two boys' FoK. A possible interpretation is that Paul saw equity for students as best achieved by treating them equally. Therefore, he may have been frustrated by the study's focus on learning about two students, seeing

${ }^{32}$ Rangi and Papa are Māori gods, respectively the sky father and earth mother. 
this learning as ultimately insufficient or possibly even irrelevant to his decisionmaking related to the class as a whole. In this way his attitude differed from other teacher participants, who perceived benefits not only for individual students but also for the class as a whole, when pedagogical decisions took account of students' unique FoKs, as shown in this exchange:

Paul: I find the whole thing very tenuous.

Georgia: When you say tenuous, do you mean the link? to the class?

Paul: $\quad Y e a h$, I find the whole knowing two or three children more intimately, and then dragging something that we can pull to make a link, and the links that can be made may be very tenuous, and that's just me looking at what l've seen there and listening to what l've seen.

Lizzie: But maybe knowing them better is the link.

Paul: I get the knowing them better and I understand that, but then I think if I know three... my problem is that l'm looking at the big picture for me as a learner with this, and I think, well I know three children better, but there's 24 in a class.

Tom: I think that's good that you've put that out there, I really do. Like for me the question is, how can I use what I know to better engage Shea better in the learning? 'Cos he's not always fully engaged, and David certainly is rarely fully engaged. And if it's gonna make him more engaged, and the class more engaged in the learning, then it's gotta be a positive thing.

Paul: Why would it make the class more engaged more engaged in their learning because Shea, because of your affiliation with, your relationship with, and your knowledge of Shea? (TFG1, 1h.20m.06s$1 \mathrm{~h} .22 \mathrm{~m} .34 \mathrm{~s})$

Thus, it seemed apparent that, for Paul, learning about two students' FoK failed to address his hope of gaining new pedagogical strategies to raise whole class engagement.

Therefore, Paul's beliefs appeared to lie within the colorblind paradigm (Gay, 2010; Rist, 1974). Teachers who hold this worldview tend to have a lack of awareness about the difficulties for minoritized students due to issues of cultural congruence, belonging, and relevance at school. They also tend to be unaware that their own cultural identity heightens their appreciation of some students' FoK that are congruent with their own. Thus teachers who hold these beliefs provide a familiar, safe environment for those whose experiences and backgrounds align with their own, and they have difficulty recognizing the lack of cultural congruence experienced by other students in the class, and the negative implications for their schooling experience. 


\section{TENSIONS BETWEEN TEACHER AS EXPERT AND LEARNER}

Paul stated that his teacher training in the United Kingdom had given him an image of teachers as specialists with academic knowledge that they would pass on. Taking a FoK approach to learning to him therefore may have seemed incongruent with the concept of teachers as experts in classrooms. In TFG1, reflecting on the study, he said:

What we're doing here is, we have these tools, we have these expertise or learnings, but the whole idea of this is that we now want to find out what their tools are and their skills are, to help us to help them... almost making ourselves look as if we are failed educationalists. (30m.25s-31m.04s)

Thus, Paul seemed to experience tensions between his role as a learner in the study and his notions of teacher as expert. This tension offers a possible reason for his apparent tendency to assume the power position in team hui, which may have occurred at an unconscious level.

\section{TENSIONS BETWEEN PROFESSIONAL DISTANCE AND TEACHER-STUDENT RELATIONSHIPS}

The professional distance which Paul tended to maintain also can be seen as being in conflict with the TBC approach and learning about individual students' FoK. Paul explained that his initial teacher training taught him the importance of professional distance. He also believed that female teachers tended to have a greater focus on teacher-student relationships than did their male colleagues, and had greater skills in this aspect of the work.

For Paul, building relationships with his KHS students was not straightforward. He understood the importance of teacher-student relationships within the school community, but in his final interview stated that his own private nature, and family life outside the local area constrained his relationship building with students.

Paul considered that factors related to the nature of his team members may have contributed to the interaction in Team D, with which he was not entirely satisfied. When he watched footage from other teams, he thought they had more of a dialogue going on, and felt a bit cheated by this. He said he noticed that parents had contributed very helpfully in other teams. He felt that inclusion of a mum in the group could have grounded the project, provided an extra person who could remind the boys about their commitment, jolly them along and bring them out of their shells, indicating that these were not roles he envisaged for himself. Also he believed that including the mum would help him to learn more about the boys, as she might offer information, and guide them in what information to share. He wondered too whether the team would have had better outcomes if he had not chosen two closeknit friends. He speculated that if he had chosen students who were more mature, older, 
with an established positive relationship with him, there would have been more of an open dialogue. It seems that Paul did not see the work of learning about the students' FoK as having a relationship-building aspect, but wanted members of his team to bring those skills to the task.

Potentially, as well as factors he identified, Paul's interactional and relationship skills with students may have contributed to the team's processes and outcomes. When teachers work with students from a different, minoritized culture, literature suggests that awareness of oneself as a culturally located being (Delpit, 1995) and strong relationship-building skills may be necessary to develop trust (Hawk \& Hill, 2002). Thus, these factors must be in place in order to establish open sharing of personal information. This challenges Paul's view of expert content knowledge as the focus of teaching, suggesting that the people involved - himself and the students - may warrant similar focused attention. Paul himself hinted at the issue of relationship building, and his own lack of confidence in this area:

Paul: Do you have to... become expert as interviewers to be able to use funds of knowledge? Because if you get someone unlike yourself who's very articulate and very interested in what they're saying you're not going to get the response you get.

Tom: I think you're got to have an interest, for sure.

Linda: But then, I don't think we should get worried about other people outside this group.

Paul: Okay well this is just my questions for me. (TFG1, 1h.11m.49s1h.12m.18s)

At times Paul seemed to be looking for a relational technique that he could apply:

Tom: $\quad$ You just think about the Davids in our classrooms - often you might not get to (know much about his funds of knowledge) over a year, you might never get to that point!

Paul: That's one of the frustrating things about it, being a teacher.

Kate: 'Cos you could have never found that out about him, eh.

Tom: I suppose that the challenge, isn't it, how to connect to where the kid is.

Paul: $\quad$ And you get that right, and you find, not a formula, but a way that you can do that, and you're licking teaching aren't you. (TFG1, 1h.56m.20s-1h.56m.48s)

However, one anecdote that Paul related showed his understanding of the value that students placed on being cared for as individuals. He described what happened when he told Piri that his dissatisfaction with his behavior was grounded in care for him as a learner. The incident had occurred after an earlier, unrelated TPL session, 
in which the teachers learnt that Māori students valued knowing that teachers cared about them as learners.

Paul: (Piri's) the one after we had our Māori meet, and we talked about that whole idea we've got to show that we care about the learning, he's the one who was badly behaved and I took him outside and he was so grumpy, and at first I started doing the typical teacher thing, and then I twisted it around and just started trying to say look the only thing I really really care about, I said you've got to really understand me and it's genuine, is your learning. And I really really am upset that you're, you know, blah blah, and he just changed completely, and he just was a different kid... it was amazing the transformation when I turned around and started saying just care about your learning. (TFG1, 2h.25m.01s-2h.25m.46s)

A possible interpretation of Paul's anecdote alongside other data about teacherstudent relationships is that the incident he described was primarily a strategy for managing student behavior, rather than a change in his conceptualization of the teacher-student relationship. Therefore, I would argue that in Team D's processes and the related incident, Paul was unconsciously enacting long-held beliefs about teacher-student relationships.

\section{TENSIONS BETWEEN DEFICIT THEORIZING AND A FOK APPROACH}

Watching the videos the students made for him, Paul found it difficult to identify their FoK. He stated twice - after his initial viewing and again in his final interview - that the only thing he learnt from it was the boys' ability to do a kind of comedy double act, and his tone on these occasions gave me the impression that his main reaction was not acknowledgement of skill, but frustration or anger. I had the impression that Paul regarded their 'performance' as inappropriate; perhaps he saw their manner as disrespecting the serious function of the video. During collaborative reflection on the boys' video in TFG1, Paul was quiet while the others effusively expressed appreciation for the boys' comedic talents, support of each other, and Peter's articulate statements about his religion, and how it had helped him transform his life. This led me to wonder about why Paul seemed to experience more difficulty identifying students' FoK than the other teacher participants.

Closer examination of the data also raised questions in my mind about the degree to which deficit theorizing influenced Paul's views about what he was seeking to learn about the students. Reflecting after his withdrawal on what he wanted to learn, Paul commented that relationships with students in this community were particularly important, so you could begin to understand what the students faced in their lives, and avoid simply reacting to any unacceptable behavior they were presenting. Rereading his initial hopes in a final interview, he clarified that he had hoped to achieve empathy for the boys, through understanding difficulties they face at home. During 
the course of the project Paul learned that Peter sometimes stayed home to look after younger siblings. He stated he found this helpful, because it affected the way he approached Peter, not making assumptions, providing some time to comply with instructions. He noted that this knowledge did not arise from his quest to learn about the students' FoK. Piri reported that, since he had learnt about their FoK, Paul "actually comes up to us and asks if we need any help on the work or if we want to do something easier" (interview, 18-11-11, lines 686-687). Therefore, reliance on a deficit-theorizing lens potentially explains Paul's difficulties learning about the students' FoK, due to his expectation and focus on deficits and problems, rather than their resources and strengths.

In further exchanges that appeared to reinforce the possibility of deficit theorizing, Paul expressed concern about the students' motivation. He judged both Peter and Piri to be not highly motivated to learn, with Peter's motivation being especially lacking. The first time he stated this was during TFG1, after watching Piri and Peter's video with the other teacher participants. In this discussion the teachers collaboratively reflected on what could be learnt about the boys' FoK:

Paul: In class Piri is fairly keen to do well, and Peter isn't interested at all.

Tom: You don't pick that up from what they say though do you, 'cos he's talking about religious...

Paul: $\quad$ Sometimes kids will talk, and say what sometimes what they think adults want to hear, don't they. (TFG1, 2h11m41s-2h12m07s)

In this exchange, although Tom points out evidence that is at odds with Paul's interpretation, Paul's response seems to provide an explanation that aligns with deficit theorizing. I would argue that this incident, along with Paul's response to the boys' movie, suggests maintenance of his schema despite contrary evidence (Timperley \& Robinson, 2002). In his final interview, Paul reiterated his belief that the boys (especially Peter) were not highly motivated, adding that throughout his 12 year teaching career, dealing with students who were not motivated to learn what he was teaching remained his biggest professional challenge.

\subsubsection{Valued outcomes achieved}

Paul stated that he learnt nothing new about the boys from the project, apart from their ability to do a comedy double act. Although the students had expected him to learn about various FoKs from their video, they predicted that Paul would learn nothing about their FoK from their teaching session. Therefore, implementation of the selected strategies to give Paul knowledge of Piri's and Peter's FoK were largely 
unsuccessful. However, the boys perceived that Paul's attitude to their humour in class had become more positive.

Paul's hope to gain new pedagogical armour ultimately appeared to be unfulfilled, as he considered that to make adjustments to lesson planning on the basis of knowledge about a couple of students was contrived and tenuous. Both boys had also had pedagogical hopes. They envisioned that knowing their FoK could allow the development of more relevant and enjoyable lessons. Peter hoped Paul would incorporate more activities involving drawing in class time. Piri hoped he would "teach more about stuff that we like" (interview, 25-11-11, line 95). These hopes were unfulfilled. Peter and Piri reported that Paul now gave them easier work, suggesting that his ideas about their prior knowledge were not enhanced by participation.

Piri had hoped to develop a closer bond with Paul, and both boys hoped that, as a result of learning about their FoK, Paul would talk to them about their interests, and express appreciation of their skills and expertise. They stated that other teacher participants had complimented them on their movie, but not Paul. Both boys agreed that their relationship with Paul had improved "heaps" (Peter, interview 2, line 214). Peter explained that Paul's management of them had loosened up, as he had learned about them:

Peter: The relationship with (Paul) is better cause he like, knows we're the boys who like always joke around and he knows like if we pull up like. When we used to joke around and he used to think it was serious and like and used to take it serious like that's a detention or get outside. But now he knows like we joke and it's just a joke.

Piri: $\quad$ Actually meaning it like...

Peter: $\quad$ Yeah we're not meaning it we're just trying to make the class laugh and then we feel special like cause we made everyone laugh... he'll have a laugh with us, like, know it's a joke.

Piri: $\quad$ And he'll say the joke's over now boys. (interview 2, lines 218-235)

Piri acknowledged that his developing understanding of Paul's rules and what was expected of him in Paul's classroom had also had positive benefits for his relationship with Paul; thus, the improvements reported by the students were probably enhanced by other factors such as their growing familiarity with life at secondary school and within Paul's classroom culture. 


\subsection{Conclusion}

Findings illustrate divergence of opinion within the group about preferred strategies to enable the teacher to learn about students' FoK, and the role of power and status in the decision-making process. Difficulties reaching agreement about strategies were followed by implementation problems.

Paul reported minimal valued outcomes from the team's work together to help him learn about the students' FoK. In contrast, the students considered that they were enjoying some valued outcomes in the form of Paul's support in class. However, aspects of valued outcomes reported by students appear to have been generated by unrelated factors.

The nature of Team $\mathrm{D}$ findings illustrates the role of factors that essentially were undercurrents operating below the surface of the team's experience: how power influenced the team's processes, and the importance of unspoken beliefs, thoughts, and feelings. Analysis of data suggested that for Paul, participation involved challenges related to a number of significant tensions between his notions of teaching and students and the underlying conceptual framework of the study.

Findings raise questions about the degree of support that may be needed for teachers and students to participate in processes of negotiating and learning about students' FoK for the achievement of valued outcomes. 


\section{Chapter 8: Team E Findings}

\subsection{Introduction to Team E}

Team E's teacher, Tom, was a Pākehā immigrant from England. He taught English and had a senior management role at KHS. Tom did not complete the baseline data template, but stated that, before the study, his knowledge of the three students varied. He was aware of Shea because of his extroverted nature, but felt he was not working effectively with him. He had had some contact with Toby's family. He did not know David very well at all, and noted that he was "the most reserved, and least engaged prior to the project" (interview, line 31), and tended to finish tasks when kept behind after class, not in class time. Tom chose these students because he hoped to improve their engagement.

The students - David, Shea, and Toby - were three boys in their first year of secondary schooling. Shea and Toby were longstanding good friends, and they met David when placed in the same class at KHS, three months earlier. All three were in Tom's Year 9 English class. David was 13 years old, and identified himself as a Cook Islander $(\mathrm{Cl})$. At the beginning of the study, the statement he said best described him in the English class was "okay" and that his relationship with Tom was "sometimes difficult." Shea identified himself as "Māori Pākehā." He was 13 years and ten months of age when the study began. Before the study, the statement he said best described him in English class was "mostly I enjoy it and it's going pretty well." Shea perceived his relationship with Tom as "mostly good." Toby identified himself as "Greek Pākehā". Although he had had no previous contact with Tom, he was aware his older brother remembered him fondly. When the study began, Toby was 14 years old. He described his relationship with Tom as "mostly good" and stated "mostly I enjoy (English) and it's going pretty well."

There were no parent participants in this team.

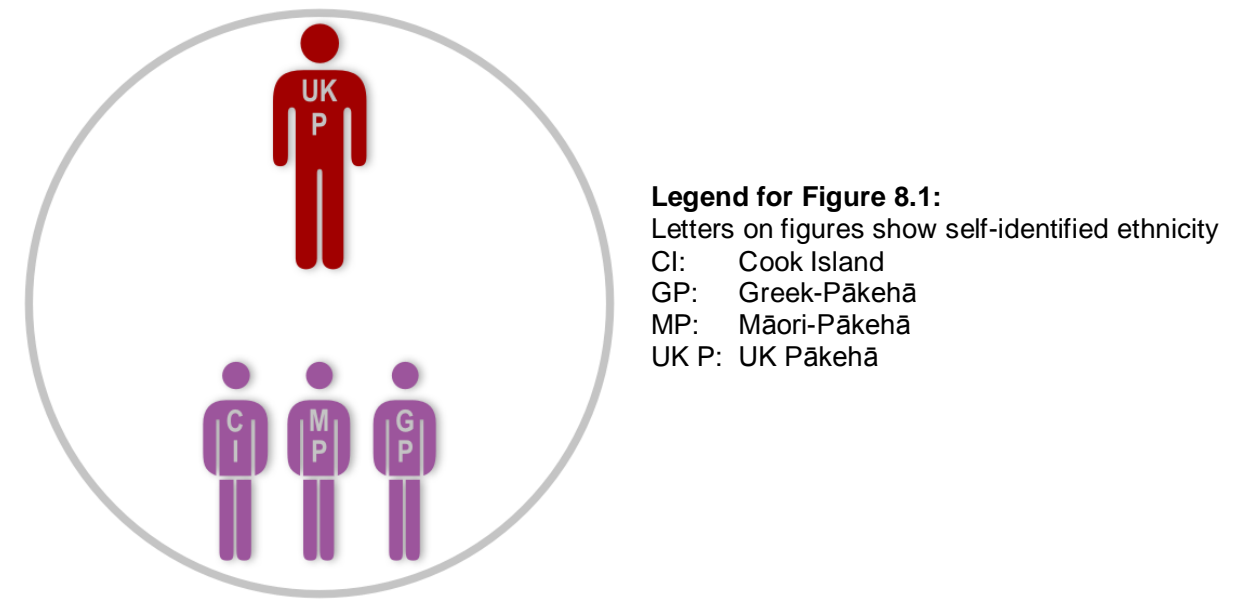

Figure 8.1: Team E participants 


\subsection{Synopsis of findings}

Toby and Shea agreed to participate in the hope they would experience a "new way to connect with the teacher" (Toby, reflection, Hui 1, lines 21-22), thus improving their relationship with Tom. David had difficulty articulating hopes, but said the project sounded cool because being in a small group would give him the chance to connect with Tom. Shea hoped Tom would learn how to "control me more easy" (reflection, Hui 1, line 18). Tom hoped participation would help him engage the boys better without getting grumpy.

Team E conducted a lengthy and complex set of strategies, to enable them to learn about each other's FoK. They met twice for a "show and tell sestion (sic)" (Andrew, What's hot reflection) and to ask each other questions. Afterwards, they decided to meet a third time, and agreed on three additional events that showcased each student's skills in turn. Although the students' observation of Tom playing soccer ${ }^{33}$ was discussed, it did not eventuate.

Team members perceived their hopes were achieved by strategies implemented. However, in their interview, the students developed new aspirations related to applying FoK in the classroom.

\subsection{Evidence by theme}

\subsubsection{Preferred approaches for teacher to learn about students' FoK}

Shea opened discussion about strategies by stating he did not want Tom to come to his house, and suggesting he could ask the students questions. Although Shea envisaged this happening in class, David preferred the small group setting. Apart from this difference, team members' ideas were very similar. They all liked the notion of meeting so Tom could learn about their FoK; and Tom wanted to extend that, to enable them to learn "the bigger picture" (Hui 2, line 137) about him. This was readily agreed. Team E's first team agreement stated they would:

Have a meeting just the four of us (Linda can come) where we will bring in photos and objects and questions, and share our funds of knowledge. We will do it on 1-2 Friday afternoons from 3-4pm. Tom will look after anything precious or any food in his office. (Team E agreement 17-6-11.pdf)

Two agreed dates were recorded, two and three weeks hence respectively.

At the end of the second team meeting (TM2), the students requested another, the following week. Thus three TMs occurred on successive Friday afternoons after

${ }^{33}$ Different names are used by participants to refer to the same game: soccer, football, and footy. 
school. At TM3, during discussion about David's dancing and drumming in a $\mathrm{Cl}$ performing arts group, Tom asked if he could watch a practice. David agreed, and Tom attended the following Monday.

In Hui 3, all team members were keen for further individual observations. Further actions were agreed:

Tom will come and watch Shea's boxing session on Monday 19 September $(4.30 \mathrm{pm})$ at Central Boxing Academy ${ }^{34}$. Linda will come too, and video-record. (Will confirm details after checking staff meeting.)

Tom will come and watch Toby play an indoor soccer game. Toby will let Tom know about game times. Perhaps Tom will play in the team for one game, if that's okay with the team. Tom could come to one training and one game.

The team will have a session together when they will view the video footage from the Cook Island dancing practice, boxing practice, and indoor soccer (3$4 \mathrm{pm}$, Friday 30 September if all the events happened by then, otherwise Friday 28 October). (Team E agreement hui 3.pdf)

Various practical difficulties affected implementation of observations (see Section 8.3.3). However, Team E students liked visiting Tom in his office, and thus by meeting informally they agreed on solutions to issues that arose.

In Hui 3, Tom suggested a class activity could involve the students teaching others about a skill or specialist area of knowledge, and made suggestions relating to their FoK. This was Tom's attempt to relate the boys' FoK directly to the oral language aspect of the English curriculum. However, all three students were opposed. Shea stated his skills were not that special, and Toby believed other class members would be disrespectful.

\subsubsection{Enablers that supported valued outcomes in Team E}

\subsubsection{Inclusive team interactions}

Participants expressed satisfaction for the way they operated as a team. Generally speaking, they enacted an ethic of care, and their respectful behavior towards each other went beyond what existed in their English class. Team members generally gave each other attention and respect during their interactions, thus creating a safe space for all.

Tom paid attention to participants' privacy and sensitivities in his introduction to the show and tell session, as well as the nature of information shared. In Hui 2 and TM1, Tom emphasized confidentiality within the group. "This is just between us. This is us getting to know each other" (TM1, 5m.30s-5m.34s). He shared his new grandfather status, which he felt uncomfortable about (believing his son too young

34 pseudonym 
to be a father), because he wanted to show the students "he had his own issues" (audio-reflection, 29-06-11, 5m.47s-5m.51s). He related the situation factually, without revealing his feelings. However, the boys' reaction showed they did not share his cultural ideas about parenthood; their treatment of it as natural and normal gave him a new perspective on the situation.

When Shea said that his dad was away, Tom's response showed sensitivity and compassion for him and his father:

$\begin{array}{ll}\text { Shea: } & \text { And my dad, he's away. } \\ \text { Tom: } & \text { Where's your dad, is that alright to ask? } \\ \text { Shea: } & \begin{array}{l}\text { Oh yeah, I don't really mind saying it eh, he's in jail, yeah, I } \\ \text { don't care. }\end{array} \\ \text { Tom: } & \text { Is he? Well, that happens. } \\ \text { Shea: } & \text { Yeah. } \\ \text { Tom: } & \text { Do you see him? } \\ \text { Shea: } & \text { Yeah, I see him like every second week. } \\ \text { Tom: } & \text { That's good. And how's he going in there, is he alright? } \\ \text { Shea: } & \text { Yeah. (TM1, 9m.11s-9m.38s) }\end{array}$

David and Toby listened quietly, thus also respecting Shea's time and personal situation.

In TM1, Tom spoke first, modeling the show and tell activity. Sharing information about his family history, Tom stated "this is a part of my history that I don't know about yet, so it's undiscovered country for me, so I thought l'd share that with you" (TM1, 4m.56s-5m.03s). This statement implicitly gave permission for students to talk about personal information in which they may not perceive themselves as experts, thus contributing to the creation of a safe environment for sharing.

Generally, conversation flowed between Tom and a student one at a time, as shown in Figure 8.2.

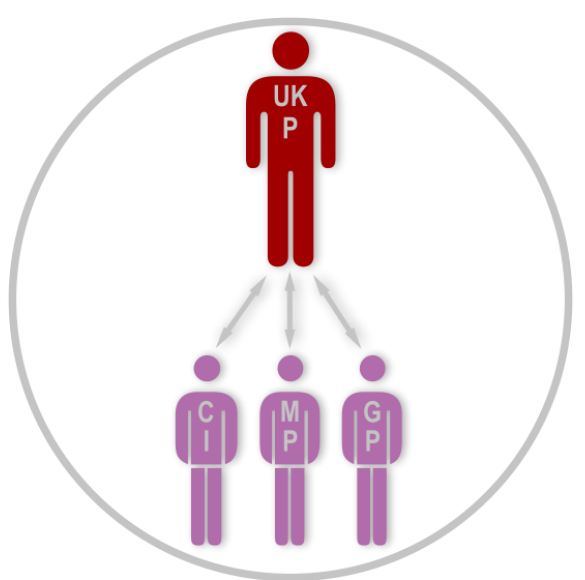

Figure 8.2: Conversation pattern in Team E 
However, on occasions individuals interjected their own personal information during someone else's turn, helping members identify connections with one another. This was a more naturalistic conversational flow, but also took attention from the person speaking. For instance, after David described his dog, Shea and Tom described theirs, and related stories about experiences with dogs. A further example occurred when David said his father was a painter, and Shea, Tom, and Toby all made comments about family members who were painters, followed by Shea sharing more about his father's work history, and Toby talking about his work experience. On one occasion, when Shea commented during a conversation largely between himself and Tom, Toby communicated his displeasure by saying "Hey, hey, hey", thus regaining his two-way interaction with Tom.

Tom regularly praised the students for things he observed. Examples are shown in Table 8.1, which also highlights the relative frequency of praise for each student. As discussed later, David's FoK proved more difficult to illuminate, providing a possible reason for discrepancies between students. Both comments to David in Table 8.1 referred to Tom's observations outside TMs, suggesting limited success identifying David's FoK in TMs. Occasionally, Tom expressed disapproval. Two examples related to the "insane" (TM1, 33m.24s) amount of hours Toby spent on Playstation and effects of Shea's lolly consumption on his classroom behavior.

Tom also enhanced inclusivity by drawing attention to things he and the boys had in common. For example, he shared a photo of himself all muddy after mountainbiking, and asked if the boys had heard of the area. Shea replied that he rode his motorbike there. Tom also showed a photo of one of his sons with a rat's tail haircut and said to Shea, "that's why when I saw you with a rat's tail, you reminded me of Henry" (TM1, 2m.51s-2m-53s). Thus, Tom made links with Shea and Toby. Although Tom's and Toby's shared love of soccer was already known, Tom explicitly drew new links with Toby by sharing stories of family members' wartime experiences and talking about online gaming. As members shared stories in TMs, everyone became more comfortable and relaxed, indicated by more naturalistic conversation structures. 
Table 8.1: Acknowledgement of students' FoK by Tom

\begin{tabular}{|c|c|c|}
\hline David & Shea & Toby \\
\hline \multirow{10}{*}{$\begin{array}{l}\text { "I'm quite proud of your work } \\
\text { boys, both of you" (To Shea } \\
\text { and David) (Hui 3, line 3) } \\
\text { "And you with your Cook } \\
\text { Island dancing" (Hui 4, lines } \\
\text { 325-326) }\end{array}$} & \multirow[t]{2}{*}{$\begin{array}{l}\text { "You're really observant, do } \\
\text { you know that Shea, and } \\
\text { another thing you're good at } \\
\text { is technology like this" (TM1, } \\
7 \mathrm{~m} .15 \mathrm{~s}-7 \mathrm{~m} .21 \mathrm{~s})\end{array}$} & $\begin{array}{l}\text { "That shows really good } \\
\text { imaginations" (To Toby and } \\
\text { Shea) (TM1, 16m.24s- } \\
\text { 16m.28s) }\end{array}$ \\
\hline & & You're pretty onto it, aren't \\
\hline & \multirow{2}{*}{$\begin{array}{l}\text { "If you can do that ... course, } \\
\text { you must be a pretty good } \\
\text { rider" (TM1, } 14 \text { m.52s- } \\
14.55 \mathrm{~s})\end{array}$} & you!” (TM1, 34.46) \\
\hline & & $\begin{array}{l}\text { "It's no wonder you're so } \\
\text { good" (TM1, 38m.51s) }\end{array}$ \\
\hline & \multirow{2}{*}{$\begin{array}{l}\text { "That shows really good } \\
\text { imaginations" (To Toby and } \\
\text { Shea) (TM1, 16m.24s- } \\
\text { 16m.28s) }\end{array}$} & $\begin{array}{l}\text { "That's absolutely wild! } \\
\text { That's amazing!" (TM2, } \\
\text { 3m.46s-3m.49s) }\end{array}$ \\
\hline & & \multirow{2}{*}{$\begin{array}{l}\text { "You've got quite a lot of } \\
\text { skills for putting the } \\
\text { powerpoint together too" } \\
\text { (TM2, 6m.28s-6m.31s) }\end{array}$} \\
\hline & $\begin{array}{l}\text { "That's quite groovy" (TM1, } \\
\text { 27m.23s). }\end{array}$ & \\
\hline & $\begin{array}{l}\text { "I'm quite proud of your work } \\
\text { boys, both of you" (To Shea } \\
\text { and David) (Hui 3, line 3) }\end{array}$ & $\begin{array}{l}\text { "You with your powerpoint } \\
\text { too, I think you really set the } \\
\text { standard" (Hui } 4 \text {, line } 323 \text { ) }\end{array}$ \\
\hline & $\begin{array}{l}\text { "That's good manners, Shea" } \\
\text { (Hui 3, line 20) }\end{array}$ & \multirow{2}{*}{$\begin{array}{l}\text { "You're a funny guy, you do } \\
\text { make people laugh" (Hui } 4 \text {, } \\
\text { line 562) }\end{array}$} \\
\hline & $\begin{array}{l}\text { "I reckon you've got real } \\
\text { knowledge there, that no-one } \\
\text { else has got" (Hui } 3 \text {, lines } \\
809-810 \text { ) }\end{array}$ & \\
\hline
\end{tabular}

Tom's strategy of making links with his own family and knowledge had complex consequences. Although it supported fluent conversation and personal connections, simultaneously it privileged his voice, directed discussion, and potentially drew attention away from students' FoK. As shown in the example below, Shea followed Tom's conversational lead:

Tom: $\quad$ And what can you do? Can you do kickflips and that sort of thing?

Shea: Yeah, I can do kickflips.

Tom: Can you? Can you kickflip a two stair? Three stair? Four stair? Do you do that stuff?

Shea: Oh, I ollie off a two stair, that's it.

Toby: I used to be able to kickflip a five stair, eh Shea.

Shea: Yeah.

Tom: Did you?

Shea: Oh I probably could, I could kickflip a four stair, or a three stair, yeah.

Tom: Well, that's good. "cos what skaters do, don't you just start with one, then go for two, then go for three.

Shea: Nah, first you start off ollying, and learning tricks first, then you start jumping off stuff. 
Tom: And can you drop in? Is that what they call it?

Shea: Yeah, in a bowl.

Tom: I tried that, and you know what happened?

Shea: You fell on your arse.

Tom: (Laughing) I did.

Shea: Yeah, l've done that three times.

Tom: It hurts, doesn't it, 'cos it's concrete. (TM1, 10m.51s-11m.47s)

In the above extract, Tom guided the scope of conversation as interviewer and Shea took on an interviewee role. This was how Tom tended to facilitate all show and tell sessions. Therefore, although students had autonomy over their content selection, exercised through choice of artefact, Tom's questions effectively mediated FoK shared. However, when Tom shared his FoK he took control of the presentation and the boys listened, occasionally interjecting a question or comment. To illustrate, Tom's show and tell session in TM1 was five minutes long (from 0m.15s-5m.15s). He showed a slideshow of photos and talked about his family, significant places, activities, and aspects of family history. During that time Shea and Toby made eight very brief utterances. For example, Shea asked two questions about photos:

Did you take these recently? (1m.40s)

Is that you? (3m.37s)

David verbalized no comments or questions, but smiled and laughed several times, such as when looking at a photo of Tom at the beach. Afterwards, comments and questions were invited, and Toby asked one question.

Therefore, this aspect of the team's interaction seemed to echo classroom power relations. I felt another risk of this conversational strategy was to inadvertently set a standard for judging students' skills, but the students' response did not bear out this concern. At times, Tom appeared to express approval of students' experiences and reveal assumptions, as shown in the following two extracts:

Tom: I think they're really good for families - animals - teaches you lots.

Shea: Possums, possums are cool.

Tom: $\quad$ And it's good for you to have to deal with all the things around them. (My daughter)'s cockatiel died, so we had the funeral, and buried it, that sort of thing, and that's really good for her. (TM1, 12m.46s$13 \mathrm{~m} .02 \mathrm{~s})$

Tom: Who do you get on with?

David: My two little brothers.

Tom: Your two little brothers. Do you look after them a bit? 
David: Yeah.

Tom: $\quad$ My boys do that. They pick up (my daughter) from school, things like that. It's a good thing to have responsibilities, to be responsible. (TM1, 20m.54s-21m.17s)

It appeared that provision of space allowed students to share FoK in greater depth, as shown here, when Tom did not ask follow-up questions:

Tom: So how long have you had the possum for?

Shea: We did have the possum. I dunno, it somehow escaped. Oh no, my mum let it go, 'cos it was getting older. As they get real old, they start to get real grumpy and nasty, so she let it go. (TM1, 13m.08s$13 \mathrm{~m} .32 \mathrm{~s})$

In a rare incident which included disrespect (by Shea) and assertiveness (by David), Shea put down David, then quickly identified his remark as a joke:

Shea: David is going to drop out next year... joking, I was only joking.

David: Chuck you on the ground.

Shea: Norman, I think Norman is. (Hui 4, lines 647-651)

Thus, Shea responded to David's rebuke for his insult by relating his comment to a student outside the team, thus re-establishing the intra-team ethic of care.

\subsubsection{Choice of strategies}

The team was generally satisfied with their chosen strategies, for four reasons. Firstly, they gave the students Tom's full attention, and allowed sharing of personal information within a safe space. Secondly, TMs provided time and multiple opportunities to engage with each other, and created forward momentum. In TM1, Tom said he would like to see some family photos, and Shea brought some along the following week. Thirdly, chosen strategies showcased members' FoK on their own terms. Finally, the reciprocal aspect of sharing was highly valued.

Toby liked the team's chosen strategies because "there wasn't much people around you or anything... so when like someone's talking they're focusing on someone, instead of like a big crowd of people talking with their mates and laughing at you" (interview, lines 504-505). Both David and Shea agreed with Toby's assessment. The students' enjoyment of one-on-one attention from their teacher was evident throughout TMs (see Section 8.3.4.2 for discussion of closer relationships developed). Toby thought if he revealed his FoK to the class, some students would "take the p out of it" (Hui 3, line 1249). Thus, the team setting was a "safe space" (Tom, Hui 3, line 1243). 
Team E's decision to hold three TMs comprising show and tell sessions and asking each other questions allowed time to consider information shared, elaborate, and ask further questions, which was particularly significant for Tom. After Shea spoke, Toby asked "Have you got a dog Shea?" (TM1, 12m.20s-12m.21s). This prompted Shea to talk about his family's dog, cats, possum, lamb, rats, fish, and horses. Similarly, Shea made several suggestions to Toby regarding things to discuss, such as his cats and his father's interest in drag racing.

Momentum was generated by the ongoing TMs, and Tom considered this was fuelled by my evident enthusiasm and commitment to the project. He commented "I would never have come up with going to see David without your knowledge of $\mathrm{Cl}$ culture and dancing and your commitment to go there and watch him" (interview, lines 459-461).

In my view the students' enthusiasm and drive also added momentum. They were explicit in Hui 3 about their preference for equitable distribution of Tom's attention across team members, met informally with Tom in between team hui, and openly expressed their anticipation and enjoyment. The students' enthusiasm and commitment to TMs was highlighted the day of a scheduled meeting, when they participated in offsite community service that ended at noon. Although they could have gone home early, all returned to school to attend the 3pm meeting.

The format of show and tell sessions and observations illuminated the boys' FoK. This took various forms, as shown in Table 8.2. However, Shea felt Toby had not made "a proper video" (Hui 4, line 426), meaning "a video that actually shows him doing something" (Hui 4, line 434). Also the students expressed disappointment they did not collectively view video footage of all members as planned, and they showed considerable interest in watching Tom play soccer. 
Table 8.2: Examples of FoK and ways they were shown

\begin{tabular}{|c|c|}
\hline Ways that FoK were shown & Examples of FoK \\
\hline $\begin{array}{l}\text { Observation of skill } \\
\text { demonstration }\end{array}$ & $\begin{array}{l}\text { David's } \mathrm{Cl} \text { dancing and drumming } \\
\text { Shea's scootering }\end{array}$ \\
\hline Sharing personal narratives & $\begin{array}{l}\text { Toby's story about his grandmother's experience in } \\
\text { Greece in World War Two } \\
\text { Shea talking about his extended family } \\
\text { David talking about the family dog and puppies } \\
\text { Toby's discussion of online gaming, and how he dealt with } \\
\text { situations when people tried to hack into his game } \\
\text { account }\end{array}$ \\
\hline $\begin{array}{l}\text { Observation of pedagogical } \\
\text { FoK }\end{array}$ & Coaching of David's Cl Performing Arts group \\
\hline $\begin{array}{l}\text { Demonstrations of skill evident } \\
\text { from presentation formats }\end{array}$ & $\begin{array}{l}\text { Toby's powerpoint and video presentations } \\
\text { Shea's cellphone photo collection and skatepark model } \\
\text { Shea's assistance to Tom in showing his powerpoint }\end{array}$ \\
\hline Communication style & The boys' enjoyment of joking \\
\hline Listening and observation skills & Shea's observation of details in Tom's photos \\
\hline Showing scars & $\begin{array}{l}\text { Toby's calm and tolerance for pain } \\
\text { Tom's sense of humour }\end{array}$ \\
\hline $\begin{array}{l}\text { Personal qualities implied by } \\
\text { verbal contributions }\end{array}$ & $\begin{array}{l}\text { Toby's assertiveness } \\
\text { Shea's perseverance } \\
\text { David's shyness }\end{array}$ \\
\hline
\end{tabular}

Finding out about Tom was highly valued by all the students. Tom shared information about his family history, family members, and interests. When he showed family photos in Powerpoint the boys all leaned forward and came closer to get a good view. Toby especially was disappointed they did not either watch Tom play a soccer game, or see a video of him playing; to Toby, this would have provided appropriate reciprocity, as Tom "got a video from us" (Hui 4, line 395) (see Section 8.3.3.2 for further discussion). Toby made three comments about this in Hui 4. In the following extract, Toby sought Tom's agreement to provide a video, supported lightheartedly by David:

Toby: It would have been way better if we got a video of Mr Gill.

David: Yeah, playing his footy.

Toby: $\quad$ Which we could still maybe do. (Hui 4, lines 385-389)

In response, Tom told them about an upcoming game, but did not demonstrate support for the boys coming to watch. In the students' interview Toby expressed disappointment again, but David was non-committal. 


\title{
8.3.2.3 Small team setting
}

All three students agreed it was good to have Tom learn about their FoK in a small group setting. Toby stated this meant Tom could focus on a manageable number of students, otherwise "he's not going to remember everything" (interview, line 329). Throughout the study, all three students (but especially Shea and Toby) took advantage of opportunities to engage with Tom on a wide range of topics, suggesting they highly valued the level of attention within a small team setting. For instance, topics raised by students included: who was responsible for different items of graffiti; their boredom from learning about the same theme in several subjects ${ }^{35}$; opinions about teachers; and concerns about students who were in trouble. David stated he enjoyed having a number of students in the team, because "you talk more, and I talk more to other people than just one teacher and one parent" (SFG3, 16m.00s-16m.09s).

Also, the students all agreed they would not have been comfortable going through the process in a whole class setting, because they considered some classmates "immature" (Toby, interview, line 347). They rejected Tom's suggestion that they teach the class something, feeling this made them vulnerable to putdowns. Therefore, within the team there was trust, a necessary pre-requisite for communication to open up. Tom also believed the pleasant setting, freedom, and autonomy added to the positive team experience:

\begin{abstract}
And for me and the boys as well I think there's been a genuine level of pleasure in the fact that you know we've got to know each other in an environment which is pleasant and you know it's just quite nice to be in a space where you can knock ideas around, you're not driven by a set curriculum into doing something and this is one of the tensions in schools now... and I think that you know that's the bit that's missing. And we've had time here to reflect on ourselves and who we are as individuals and how that meets across a marae if you like or across a divide of some sort. (interview, lines 314-321)
\end{abstract}

Tom felt the students found it easier to relate to him within a small group, and therefore the enhancement of their relationship as team members reinforced their trust in him and led them to value his support.

They always come to me when they've got an issue that's for sure, but I mean that's a testament to a relationship that probably works better in the smaller setting than it does in the class setting and certainly that's another part of the learning for me is that the boys are far more responsive in that environment than they are in a class setting. Which is not to say there aren't any positive spinoffs into the class setting because I think there are, but they still operate as student/teacher in the traditional mode, and that is a different relationship to a participatory relationship, such as the one we have in the funds of knowledge work. (Tom, interview, lines 94-100)

35 The boys seemed unaware that their class was undertaking an IS program. Instead, they perceived that teachers were copying each other's ideas. 
Shea's belief that "all the other (senior managers) hate us" (interview, line 229) seemed to verify Tom's observation.

Team members agreed spending time together within the team set the scene effectively for enhancement of relationships, which then created readiness, willingness, and commitment to individual observations:

Toby: I think they both connected on, so they were useful.

Tom: Connected on at different levels, I think that's a really good point... I think it was really important doing all that getting to know you stuff first.

Toby: Good that we did the show and tell thing, where we brung whatever in.

Tom: Yeah, we all got to know each other on a level: you got to know stuff about me, I got to know stuff about you, thought it was good.

David: Yeah. (Hui 4, lines 664-688)

In Tom's interview, he reflected the commitment of personal time that he made was significantly beyond his norms, and attributed this to my evident commitment. However, I would argue that the collective commitment of all members and ongoing nature of team interaction collectively generated a spiral of forward momentum.

All the boys were glad to be in a team without parents, because it offered them the opportunity to participate on their own terms. Shea and Toby worried parents could have brought up embarrassing "baby stuff" (Toby, interview, line 401) that would not reflect their current selves. David stated "I wouldn't be able to talk in front of my parents" (interview, line 385), and Shea and Toby agreed it would have been "awkward" (Shea, interview, line 377). However, in SFG3, David stated he thought parent participation would have been helpful.

\subsubsection{Barriers to valued outcomes in Team E}

\subsubsection{Drawing out all voices}

Various factors affected expression of ideas and information, including students' confidence, personalities, prior experiences, and questions asked.

One challenge arose from differences between students; Toby and Shea were relatively confident and extroverted, and were long-standing friends, whereas David was very shy and withdrawn, particularly at the beginning of the project.

Toby expressed his hope, when the study began, that participation would enhance his connection with Tom, and analysis of conversation suggests he took active steps to achieve this. For instance, he took initiative to engage Tom in conversation. For 
example, the formal start of Hui 4 was delayed by conversation initiated by Toby about a soccer training academy he attended.

Toby's conversational initiative demonstrated confidence and cultural capital, resulting in his FoK and ideas being clearly expressed. Shea was also relaxed in conversation with Tom; both boys often made elaborated remarks:

Toby: $\quad$ One cat died when it was 23 years old, and another one died when it was twenty.

Tom: That's really old for a cat. How did you manage that?

Toby: Oh, I didn't feed it milk. Apparently you know they say milk's good for their bones, but it builds up cholesterol and blocks their arteries. That's why... some cats die young. So we just fed the cats water, and there's this drop you put into their water, it's like a vitamin.

Shea: $\quad$ We put in, our dogs... every week, every Friday, we'll put an egg in with its meal.

Tom: Is that right?

Shea: Yeah, 'cos it makes the coat thicker. (TM1, 41m.36s-42m.23s)

In contrast, early on, David found it very challenging to express his ideas, and did so only when prompted. In SFG3, he stated it was scary initially to ask Tom questions in the team, and too hard to disagree with Tom's ideas. Tom observed "it's been quite hard to draw him out... far more than the other two, and even to get the stories straight" (TFG1, 1h.53m.28s-1h.53m.35s), and found that providing space for David was helpful. David's verbal contributions were brief - he tended to use non-verbal communication, such as shrugging. Both Tom and I rephrased questions and prompted him heavily to encourage expression of his ideas.

The scope, depth, and breadth of conversation were affected by the nature of questions asked. In early TMs, Tom's questions and comments were influenced by his prior knowledge of the students:

Tom: I wanna know more about your Cook Island thing... I wanna know more about Toby's (soccer) and Shea's motocross and the other things that he does, his boxing. You do motocross?

Shea: Yeah, l've raced about four times.

Tom: You mentioned that to me once I remember. (Hui 2, lines 97-104)

TFGs provided a useful opportunity for Tom to observe and reflect on his role in TMs, leading him to experiment with techniques:

There are bits about myself I became aware of, about making more space for the boys and not talking too much and all the sorts of things that we as teachers do all the time... but you know that's the reality and that's quite good to have that reminder. (interview, lines 365-368) 
Responses to closed questions varied depending on to whom they were addressed, because David tended not to elaborate information. Some closed questions identified experiences David had not had, not his FoK:

Tom: Have you been pig hunting ever?

David: Nah. (TM1, 18m.07s-18m.09s)

Tom: You got any war stories, David?

David: I don't know. (TM1, 40m.43s-40m.46s)

Tom: Have you ever had a go on a car or a bike?

David: No. (TM2, 14m.06s-14m.10s)

Tom: $\quad$ Go to the cinema?

David: Ah, nah. (TM2, 38m.48s-38m.50s)

An unintended consequence was to highlight differences between team members, rather than identify connections.

Open questions worked well to highlight Toby and Shea's FoK:

Tom: So tell me about the hacking thing. And how do people hack you? And what's the point in hacking?

Shea: They have this thing called...

Toby: Hey, hey, hey. (smiles at Shea)

Shea: Okay, say it then.

Tom: What do they do, Toby? Why do they do it?

Toby: They have this jail break thing, and what it does is they search someone's name, and then they go into this hack lobby, and they put in the person's name, and it tells them the person's email address, tells them all their things like that. But I have this security thing built into my Playstation...it's this firewall, so if anyone tries to hack into my account, it tells me, and blocks them, and I have this reverse sympathy thing, so while it blocks them, while they're trying to hack me, I can hack them back if I want to.

Tom: But why do they hack you? What are they after?

Toby: 'Cos they're jealous of my account. 'Cos you start off at level one, and by the time to get to level seventy, you have all the guns and that.

Tom: So they can take your guns if they hack you.

Toby: No, they can only take my account.

Tom: They can only take the whole account?

Toby: They can't take things off the account, they can only take the whole account. The highest level on the game is ten procedural seventy, 
and I'm ten procedural seventy, and lots of people want to be that level, so they always try and hack the account.

Tom: Oh, so you're the top prestige in the game!

Toby: Yeah.

Tom: Well that's pretty full on! (TM1, 31m.36s-32m.55s)

As TM1 progressed, Tom seemed to allow more space for students to take conversational initiative. However, David did not take advantage of these spaces. He was fully attentive and participating actively as a listener, but tended not to speak. In TM2, Tom continued his attempts to engage David in conversation, and his directed questions eventually led to David talking about his parents and how they met. Tom shared the story of his child's broken arm, and suggested sharing accident stories. David's accident story was:

I was riding on my scooter and there was this big-as pole, and I crashed into it and fell face first into the ground. Yeah. Hit the pole. (TM2, 27m.56s-28m.08s)

Although David's face showed enjoyment, somehow the story fell flat in the telling, and no comments or questions were forthcoming from others. Similarly in TM3, Tom invited David to "tell us a story" about performing for an audience with the $\mathrm{Cl}$ group. David responded excitedly, talking animatedly and smiling:

Ah, we got there, there was only a little bit of people, but then halfway through, it's like, heaps in a line. The line went through the door, long as, and, yeah. (TM3, 7m.24s-7m.38s)

As fieldwork progressed and the team spent more time together, David's body language and increased contributions showed he was more comfortable and relaxed, and David confirmed this in SFG3. He began to volunteer comments, ideas and questions. For instance, in Hui 4, David's unsolicited remarks included asking what shoes are worn for ten-pin bowling, reporting that other students got angry at him after Tom came to the $\mathrm{Cl}$ group practice, and talking about a class visit by corrections officers.

Both Toby and Shea shared some prior experiences that may have influenced their relative confidence to express themselves. As primary school students, both discussed ideas for implementation at the school as Student Council members. Also, both had considerable outside school contact with adults: Toby in his sporting activities, and Shea as the only child in his household.

David's household included his parents, grandfather, and six children. In the $\mathrm{Cl}$ group he belonged to, adults were in leadership roles. David's previous experience with teachers was described in this comment "We just had to go with it... what the teacher said" (interview, lines 448-450). I also knew, from living there myself, that in 
traditional $\mathrm{Cl}$ society, groups tend to operate on a hierarchical basis, giving much respect to elders. It was not the traditional $\mathrm{Cl}$ way for young people to take conversational initiative, so for David moving between this world and his team role may have resulted in cultural dissonance. However, data collected did not identify the nature of relationships in participants' families. Nevertheless, for David, the nature of interaction with his teacher that occurred was beyond his prior experience.

\subsubsection{Implementation issues}

Despite agreement about strategies, implementation involved various difficulties. Also, a conflict of interest emerged for David when Tom attended his $\mathrm{Cl}$ group practice.

Some ideas about activities Tom might observe could not happen. For instance, Tom and Toby liked the idea of Tom attending Toby's soccer game, but the season had ended. Shea asked "why didn't you just go and watch him at the academy" (Hui 4, line 227), but Tom and Toby knew training sessions were not open to outsiders. Therefore, this preference was not implemented.

Similarly, when Toby suggested in Hui 3 that the boys watch Tom in a favorite activity, as he had for them, the students' request for transportation made implementation challenging. Although I provided transportation to Shea's scootering venue, I did not offer it this time, and no further discussion of transport occurred. Later Tom seemed to retract his invitation, saying "I don't think it's about me; you don't need to come and see me" (Hui 3, line 1277). Thus it seemed the notion of watching Tom was too problematic, and he considered other strategies would be better in progressing project aims.

Team members met informally between team hui to address implementation challenges, and resolved issues by working out alternatives. For example, Tom could not either play in, or watch, Toby's indoor soccer game as hoped, so members agreed on a special TM at which Toby could share a video he made of trick kill shots he used in PS3. He also showed a video from YouTube of boys doing trick moves with soccer balls.

In a further example, soon after Hui 3 Shea gave up boxing, because of transport difficulties. The team met informally and agreed Shea would demonstrate scootering. After the special TM to watch Toby's videos, the whole team watched Shea's demonstration, but rain shortened the event. A week later, Shea gave a lengthy demonstration for Tom and David. 
Elapsed time between decisions and implementation, shown in Table 8.3, also highlights organizational challenges. Table 8.3, Row 2 shows a strategy the team anticipated implementing within two weeks was completed in eight weeks. However, commitment to implementation is shown by strategy completion despite the unexpectedly elongated nature of the project, and looming closeness of the school year end, in December. The only agreed strategy not implemented was a TM for viewing video footage from Tom's individual observations of students.

Table 8.3: Dates of strategy decisions, planned and actual implementation

\begin{tabular}{lllll}
\hline & Strategy & Decision & $\begin{array}{c}\text { Planned } \\
\text { implementation }\end{array}$ & $\begin{array}{c}\text { Actual } \\
\text { implementation }\end{array}$ \\
\hline 1 & $\begin{array}{l}\text { Teacher's observation of } \\
\text { David at Cl group practice }\end{array}$ & 5 August & 8 August & 8 August \\
\hline 2 & $\begin{array}{l}\text { Teacher's observation of } \\
\text { Shea at boxing training* }\end{array}$ & 9 September & 19 September & $\begin{array}{l}4 \text { November and } \\
11 \text { November }\end{array}$ \\
3 & $\begin{array}{l}\text { Team viewing of video } \\
\text { made by Toby }\end{array}$ & $\begin{array}{l}\text { Informally decided } \\
\text { early November }\end{array}$ & 11 November & 11 November \\
4 & $\begin{array}{l}\text { Team viewing of video } \\
\text { footage from teacher's } \\
\text { observations }\end{array}$ & 9 September & $\begin{array}{l}30 \text { September or } \\
\text { 28 October }\end{array}$ & (unimplemented) \\
\hline
\end{tabular}

* Note: The observation eventually conducted was of Shea scootering, not boxing.

In contrast, implementation of initial TMs was relatively smooth; all three TMs occurred on agreed dates with full attendance. Encouraged by Tom, all members except David brought artefacts - photos on a cellphone, images displayed in powerpoint, and treasured possessions, a team cap and games console. Artefacts were discussion prompts, providing team members with things they could see, touch, comment on, and ask further questions about. However, David stated he forgot to bring artefacts to TMs, which he said made it harder for him. I also observed David used less eye contact when relating stories and information, and provided fewer details to enhance interest for others. Several times in TMs I observed Tom looking at him in a perplexed way, seemingly wondering how he could learn about this mysterious boy. However, observing David in his $\mathrm{Cl}$ performing arts group practice was more successful for Tom as a learner and manageable for David. Because he participated in normal fashion, this was not demanding of him, and it was culturally congruent. Also, Tom interpreted David's noticeable co-operation and unusually smiley countenance earlier on the day of the observation as signs of his happy anticipation.

However, Tom's attendance at David's $\mathrm{Cl}$ group practice created a conflict of interest for David, which he found difficult to resolve. Although David was pleased that Tom came, he stated "everybody got angry at me afterwards...for letting (him) 
come" (Hui 4, lines 334-338). David noted "most of the people (in the $\mathrm{Cl}$ group) come to the school. One was expelled and the other ones are still coming" (Hui 4, lines 346-347). One student also complained to Tom at school about his presence there. Therefore, to some students, Tom was a school manager who disciplined them, and apparently for them his presence was an invasion of privacy. David stated in Hui 4 that, given the choice, he would do it again. However, in his interview, David agreed with Shea and Toby that some things should be kept private, thinking of the privacy of other students in the $\mathrm{Cl}$ group.

\subsubsection{Valued outcomes achieved}

\subsubsection{Knowledge of each other}

Tom began the study with some prior knowledge of the students and some incorrect assumptions, but as the study progressed, his awareness of them as individuals became more enriched. For instance, new knowledge included Shea's expertise with animals, scootering, and technology, as well as his cultural knowledge. Examples of new knowledge related to David were $\mathrm{Cl}$ performing arts and coaching practice, as well as home and church practices. Tom's identity as a father gave him ideas about the students' interests, and knowledge about these activities (such as skateboarding and online gaming). Toby's FoK had significant alignment with Tom's own sons, but this was less so for Shea, and especially for David. General answers to questions Tom asked in class led him to believe all students had Facebook accounts and computer access, but he learned that access, skills, and confidence with technology actually varied.

For each student, Tom gained an enduring image. "The image for Toby for me is that (drag racing) car, just the picture of the car and how that changed our perception of him and what we knew about him, it was quite funny" (interview, lines 349-351). For David, it was "the dance teacher whispering in his ear" (interview, lines 347-348). For Shea, it was "that picture of him manualling down that whole playground which is a metaphor for how he's got through the year" (interview, lines 113-114). Some aspects of Tom's new understanding were not explicitly stated, such as understanding the boys' joking communication style, but his awareness was apparent through his changed actions (see Section 8.3.4.3).

Tom's expression of an enduring image for each student raises questions about the depth and breadth of understanding gained of each student. Although data highlighting students' FoK (see Table 8.1) provide some indication of Tom's potential learning, this is insufficient to draw accurate conclusions. Lack of time to 
review video-recorded data relating to students' FoK was a problem for Tom as for other teachers.

Some items discussed in TMs raised Tom's consciousness about his own cultural identity, as well as understanding of students' cultural backgrounds. For example, notions of family differed between Shea and Tom. In TFG1, Tom reflected that the students' nonchalant response to the information that his teenage son was a father highlighted that, for them, this was not unfamiliar or undesirable. Also, Shea's perspective challenged his ideas about the scope of family and how families operate:

Tom: $\quad$ And so does your mum still go and see him too?

Shea: Just me and my step-mum. Oh 'cos my dad's girlfriend is like my step-mum.

Tom: $\quad$ So you still see her. And brothers and sisters? I didn't catch that bit.

Shea: Oh yeah, l've got a little brother named Sam.

Tom: Is he half-brother or full brother?

Shea: Oh, he's half, but yeah.

Tom: But he's your brother and he lives with you.

Shea: He doesn't live with me.

Tom: Doesn't he?

Shea: He lives with my step-mum. (TM1, 9m.49s-10m.19s)

Because of a combination of factors such as David's reticence, shyness, and lack of assertiveness, Tom's limited success with various questioning techniques, as well as the assistance that artefacts and prompts to each other provided Shea and Toby, it was relatively difficult for Tom to gain knowledge of David at TMs. However, learning about David's FoK from observation was easier to implement and added considerably to Tom's knowledge. David stated that Tom's knowledge of him progressed from $3 / 10$ to $8 / 10$.

For the students, getting to know Tom was an important aspect of what they did as a team. Discussing his feelings about Tom's class before the study, when he reported their relationship as sometimes difficult, David said, "I never really knew him" (interview, line 673); Toby added it was important "you both understand each other" (interview, line 686). David also stated he valued getting to know the other students. 


\subsubsection{Closer relationships}

Tom believed investment of time to get to know each other showed the students they were valued as individuals, leading to improvements in their classroom learning behaviors (see Section 8.3.4.3). He also stated:

I think the benefits come from developing a trusting relationship based on bettering their knowledge of each other in order to be able to educate each other. (interview, lines 304-305)

The confidence and trust which all the students gave Tom, and their evident enjoyment of team time, testify this indeed occurred. I also argue that, because the boys had hoped for a closer relationship with Tom, their continued desire to spend time with him over the course of the study, and beyond completion of the school year, evidenced the successful development of their relationships. Gains made are evident from comparison with baseline data: initially, Toby and Shea reported their relationship with Tom as "mostly good" and David stated it was "sometimes difficult." In their interview, all three stated their relationship with Tom was now "very good."

Toby explained the change in the boys' relationship with Tom:

Toby: Now that he knows us better now he treats us like normal kind of, like his friends. Nah. (laughs)

Linda: So friends is not quite right?

Toby: Nah, he treats us, not like friends, he just treats us like, still like students and that, but he just doesn't get so angry 'cos he understands us better, like he understands like our sense of humour, if we say something he might not understand he knows that's just part of what we do or part of us. (interview, lines 203-209)

Therefore, Tom's enhanced understanding of the students informed his approach to managing them in the classroom (see Section 8.3.4.3).

At various times the team's close bond was evident, and this was most important for the students. Toby stated "you know everyone better, you're not just shy around them, you're open... (with) everyone in the room" (Hui 4, lines 566-574). Tom responded, "I agree with that... I think we all have a sense of belonging as a result of (the project), including Linda" (Hui 4, lines 581-582). The boys' wish to have a final team outing highlighted the bond they felt. When the team went ten-pin bowling after their final hui, the students were enthusiastic about the outing and wanted it to be lengthy:

Linda: I said to your parents l'd deliver you home between 5.30 and six.

Toby: My parents don't care what time I get home.

Shea: Neither does my mum! My mum said, 'Be home by nine,' and that's it! 
Toby: $\quad$ Mine said 'be home by one.'

Tom: One game's an hour, so we're gonna do a game, and that's it.

Linda: I said that, so I have to stick to what I said.

Shea: You can ring our mums! Look, l'll ring home right now! Look, I'm ringing Mum up! (Hui 4, lines 118-132)

Although the boys' enthusiasm for ten-pin bowling was unsurprising, I was struck by their wish to spend extensive time on a Friday after school with their teacher, particularly since Shea and Toby both participated in many activities outside home. For David, the event was rare; he had not been ten-pin bowling before, and he did not often participate in activities outside the local area.

Tom's relationships with each boy improved, to differing degrees. Tom noted his relationship with Toby, with whom he "shared similar cultural capital" (interview, lines 138) deepened. However, he considered that relationship gains with David and Shea were much greater and more "educationally advantageous" (interview, line 132) (see Section 8.3.4.3).

Various factors in Toby's communication with Tom demonstrate their closer bond. From the outset, Toby was confident and quick to offer his ideas, but some changes were evident over time. For instance, Toby enjoyed making humorous remarks; over the course of the study, he also began to make jokes shared exclusively with Tom, related to their shared love of soccer.

Tom fostered his bond with the students by learning about them and showing appreciation of their FoK, and sharing personal information about himself, attaching less importance to the latter. In Hui 4, Tom shared information about an upcoming game and the boys showed interest in watching. Nevertheless, Tom did not encourage them to attend:

David: That's after school finishes, eh?

Toby: Artificial pitch.

Tom: From one to 2.30 .

Toby: We could come and watch your game.

Shea: And we could come to your house afterwards for dinner. (Hui 4, lines 400-410)

As discussed earlier, the students highly valued going through a mutual, reciprocal process of learning about each other with Tom. Accordingly, Toby expressed an idea related to a possible improvement to the team's process: "It would have been way better if we got a video of Mr... (he) got a video from us" (Hui 4, lines 385-395). 
Referring to the relative sharing or themselves by the students and Tom, Toby stated "we actually found it a little bit unfair" (interview, line 690). However, neither David nor Shea indicated agreement.

Toby and Shea had known each other for years, and in the course of the study they developed closer relationships with David as well. Toby stated "I didn't mind saying anything around David either" (interview, line 475). The boys' new bond as a group was evident when they expressed interest in accompanying Tom to individual observations; however, the only occasion this happened was when Shea demonstrated scootering. The three boys also visited Tom together in his office many times, especially if one of them was in trouble, or to discuss an aspect of the project.

An aspect of the team's identity that Tom believed strengthened their bond was their shared masculinity. He reflected:

I felt it was a positive space for us to be male together and while you've probably mothered us a bit as well with the way you've provided us with cake and what have you, you know that's been a nice thing too because it's been, it's helped the whole staging of the thing. You know we've been observed, we've been watched, we've been recorded, we've been fed, you know there's lots of things that have been done to keep us happy as well in our masculinity if you like. And you know that's quite crucial and certainly for boys, and you know the boys always ask are we having pizza today or what are we going to eat today, and that's a very boy thing isn't it... it has been a very unifying thing for us as boys and a man, you know and boys becoming men. And I think we need far more of that in our society not just our schools but you know we need to find more ways to do that for each other. (interview, lines 277-291)

One negative consequence arising from the closer relationships was increased vulnerability, and Tom described an incident that was "one of the hardest things" (Hui 3, line 188) for him arising from the study. The incident happened one day when he told Shea off in class. Shea responded by saying "You're no good to me and my family" (Hui 3 , line 214). Tom interpreted this as Shea saying 'you're not in my family anymore,' indicating he had lost his place in Shea's inner circle of trust, and felt "pretty stink" (Hui 3, line 206). Discussion in Hui 3 about the incident, which Toby also remembered, revealed Shea was quoting a line he had heard, deflating its significance.

Tom perceived there were further benefits related to his relationships with members of the $\mathrm{Cl}$ community beyond Team E. Although one student complained to Tom about his presence at the $\mathrm{Cl}$ group practice, Tom's experience of his visit was different to David's. Tom perceived his presence led to improved relationships, with David and other $\mathrm{Cl}$ students and parents:

I think that us going there was quite important to him, and while he's never said that, there's a much greater ease in his relationship with me since, and it 
also firmed up my relationship with Thor ${ }^{36}$ too funnily enough, who I didn't go to see but was also there, and it also made things a lot easier between me and Teremoana's mum who was there. (interview, lines 197-201)

Also, when Shea was stood down from school during the study, his mother sought a meeting with Tom, leading Tom to conclude "she felt that there was a good relationship there" (interview, line 246).

\subsubsection{Effective teaching and learning behaviors}

The improved relationships between team members led to more effective learning behaviors for the students. Tom observed "(this work) is definitely central to how prepared they are to do the difficult things" (post-TFG1 reflection). In his interview, he explained that in his view:

Knowledge of each other has at least helped to develop a preparedness to embrace an inquiry approach, for example, to nuclear power, or a thinking about viewing text using someone else's semiotics, and someone else's language, and someone else's capital, because there's been an interest paid and an investment paid in the possums or the Cook Island dancing or... the cars or whatever. (lines 177-181)

Toby also alluded to this, stating "There hasn't really been a change in the class; there's been a change in us three" (interview, lines 522-523).

Although the boys also acknowledged gains in engagement and work output (described below), in their interview they expressed a wider vision of how they would like to see FoK applied in classrooms. Shea stated:

Give us the work that he knows that we can do. Like don't give us baby work, give us more work. (lines 72-73)

Also, they wanted teachers to "put stuff that we like into our work to make us do good work" (Toby, interview, lines 62-63); they saw their FoK as a potential tool for teachers to "stop boring us" (Shea, line 145).

Educational gains differed for David, Shea, and Toby, although Tom reported "an improvement in their work rate" (interview, lines 56-57) for all three. Both David and Shea began to demonstrate more effective learning behaviors, whereas Toby maintained previous work practices. Although no claim is made linking academic achievement to the study, achievement gains over the school year were greater for Shea and David than for Toby. Tom concluded the project was "most beneficial educationally for (his) relationship with Shea and David" (interview, line 125). In this section I highlight some differences between students, to illuminate possible reasons for variation in outcomes.

36 Thor also participated, as a member of Team A. 
Before the project, Toby demonstrated more effective learning behaviors in class and had higher levels of achievement than Shea and David. Tom noted:

(Toby) already has, in Bourdieuian senses, high cultural capital: he connects strongly to the European framework, he understands exams, the knowledge he's got of powerpoints, of how to create movies, all the stuff that we've seen over the meetings, is all stuff that will get him a good job and that will get him you know where he wants to go in life, so that he can afford his toys, and he can afford the things that he wants. (interview, lines 126-130)

One change related to David's verbal involvement in class. Before the study, David was extremely withdrawn. Tom noticed David volunteered ideas in class for the first time after the study began, and that the boys became noisier in class. David affirmed "we talk more often" (interview, line 225). In their interview, Shea commented "you can't stop (David) while he's in class" (line 456). David also reported Tom now helped him more in class, because he now asked for help more.

For both Shea and David, improvements in behavior and work were evident. Shea considered his behavior and work output in class had improved. Tom agreed, saying "yes, you do more work, don't you, that's the biggest change" (Hui 4, line 539). David reflected that he also did more work, and Tom confirmed "Yes, you do more work, don't you? Miles more than you used to, and some quite good work too" (Hui 4, lines 593-594).

All the boys agreed Tom became more tolerant of them in class, and less reactive:

Toby: He's probably actually been a bit softer on us.

David: $M m$.

Toby: $\quad$ Because he feels, he knows it's... like not so strict on us like he was at the start of the year.

David: Yeah.

Shea: Not as grouchy as he used to be. (interview, lines 195-201)

Tom confirmed "my tolerance for Shea is now much wider" (interview, lines 108109).

Tom reported the boys raised their achievement level in English over the period February - November 2011 (fieldwork was between June and November 2011), with David and Shea making more marked improvements. David's performance in English progressed from curriculum level 2 to level $4^{37}$; Shea's improvement was from level 3 to level 4. In comparison, although Toby's skill levels rose, his gains were within Level 4, from 4B to 4P. In congruence with Toby's assessment results,

37 The levels referred to are levels of achievement defined within the New Zealand Curriculum Framework. Each level comprises four sub-levels e.g., level 4 includes 4B (basic); 4P (Proficient) and 4A (advanced). 
Tom commented Toby's work "has been pretty steady throughout the year" (Hui 4, line 542), and Toby concurred.

\subsection{Conclusion}

Shea, David and Toby valued being in a team with their teacher and without parents. It provided them teacher attention and a safe space to share personal information. Team time consolidated trust, developed bonds between members, and led to observations of individuals.

Identifying David's FoK was more challenging in TMs, where his verbal contributions were infrequent and brief. However, observing David in his CI Performing Arts group allowed Tom a deeper perspective of his FoK.

Student-teacher relationships improved and benefits extended to the classroom, affecting behaviors of both teacher and students. Academically, David and Shea achieved more progress than Toby, but data were insufficient to link achievement to the study. The boys' vision of applying FoK to set work that honours students' competency and differentiates learning to enhance relevance and engagement identifies possible next steps. 


\section{Chapter 9: Cross-team Findings}

\subsection{Introduction}

Examination of team findings revealed three overarching themes that underlie the structure of presentation of cross-team analysis within Section 9.2. The themes are: team structure and process, strategies through which teachers can learn about students' FoK, and valued outcomes. Appendix C shows how these three themes arose. The chapter highlights similarities and differences across teams' approaches and experiences. Valued outcomes across teams are related back to participants' hopes, to allow assessment of valued outcomes according to expressed hopes. In Section 9.3, findings related to the actual experience of conducting the TBC approach are presented, with a focus on supportive and challenging factors, including decisions related to my role as researcher.

\subsection{Overarching themes from findings}

\subsubsection{Team structure and process}

The team size and structure was originally planned, as part of the TBC approach, to allow expression of multiple perspectives relating to strategies and valued outcomes achieved, but benefits arising went beyond this hope. The small team size was valued by student, parent, and teacher participants across teams (Hogg, 2012), for various reasons. Reported benefits included:

- Input from all participants was valued;

- Trust was established through inclusive team processes;

- A new model of interaction between students, parents and teachers.

Findings confirm students and parents have expertise and can make valuable contributions to decisions about strategies for teachers to use. Students in most teams reported barriers they overcame to develop their confidence to participate in the team process. Several students reported that initially team membership was challenging, because it involved participants who did not normally work together collaboratively. For example, Briar normally kept home and school separate, but her initial concerns arising were shortlived. Jacob found the "adulty" (interview, line 339) opportunity exciting and challenging. However, all students who reported these tensions stated their anxiety eased as they "got used to it" (Briar, SFG3, 12m.01s12.03s). One factor that may have assisted students' confidence to participate was relevant FoK. Students in Teams A, B, C, and E had FoK regarding collaborative discussion with adults, gained in various settings. Examples were family meetings (Jacob and Troy), administrative meetings (Dan), involvement with adults in sports 
and other activities (Briar, Troy, and Toby), classes for gifted and talented students (Briar), and involvement in student councils (Shea, Toby, and Sonny Bill). In Team A, students' contributions towards collaborative unit planning highlighted their ability to support teachers to plan motivating units of learning. Expression of students' perspectives regarding strategies allowed teams to identify, consider, and select strategies students thought motivating and manageable.

Parents drew on multiple aspects of their identities and FoK to contribute ideas relating to classroom strategies. Ses (Team A) and Sandy (Team C) drew on their prior experiences as school students to think of strategies. Sandy also speculated about current students' perspectives related to suggested strategies, and her ideas were validated when strategies were implemented. Sandy also drew on her FoK as a mother, critiquing school practices and offering suggestions, thus making links between the study and other areas of school life, such as pastoral care and reporting. Pearl drew on FoK arising from her identities as a mother, wife, and teacher. In Team B, parents and students drew on their FoK related to negative experiences of schooling. These FoK enabled and influenced contributions.

In all teams except Team D, an inclusive team environment allowed establishment of trust between members. Diverse actions were successfully used to create inclusive environments in Teams $A, B, C$, and $E$, highlighting a range of possible effective approaches. Each team's communication had different features, and subsequently a different character, as shown in Table 9.1 .

Table 9.1: Teams' approach to achieving inclusivity

\begin{tabular}{|c|c|c|}
\hline Team & $\begin{array}{l}\text { Character of team } \\
\text { interaction }\end{array}$ & Strategies \\
\hline$A$ & Supportive & $\begin{array}{l}\text { Flexibility; } \\
\text { Positive verbal responses to information and ideas; } \\
\text { Positive non-verbal responses to information and ideas; } \\
\text { Reciprocity. }\end{array}$ \\
\hline B & Whanaungatanga & $\begin{array}{l}\text { Direct, honest statements about thoughts and feelings; } \\
\text { Focus on students' current goals and needs; } \\
\text { Privileging of students' perspective. }\end{array}$ \\
\hline C & Collaborative & $\begin{array}{l}\text { Collective brainstorming; } \\
\text { Reciprocity; } \\
\text { Support given to youngest member; } \\
\text { Care for others' perspective and needs. }\end{array}$ \\
\hline$E$ & Bonding as males & $\begin{array}{l}\text { Seeking connections; } \\
\text { Attention to privacy and sensitive information; } \\
\text { Teacher modeling; } \\
\text { Reciprocity; } \\
\text { Listening to what students wanted to talk about; } \\
\text { Acknowledgement and praise. }\end{array}$ \\
\hline
\end{tabular}


This table highlights distinctive features of teams' identity and interaction. Other strategies also contributed to the establishment of trusting relationships within teams; for instance, participants in all teams reported they were listened to and respected. Many participants across teams expressed appreciation for the "safe space" (Tom, Hui 3, line 1243) offered by team settings. Reviewing videotaped data highlighted a range of behaviors that could be expected to contribute to these positive feelings - active listening strategies (such as eye contact, attentive body language, and encouragers) were used by participants and me. Also, as hui facilitator, I frequently summarized participants' ideas and opinions, and asked for clarification.

Initially, the research design aimed to allow consideration for multiple perspectives about strategies for teachers to learn about students' FoK. Findings from a range of teams highlight participants' satisfaction with the democratic process undertaken. The opportunity for students and parents to provide input to teachers also allowed them to express the high value they placed on schooling. Strategy suggestions were made by student, parent, and teacher participants. Teams $B, D$, and E implemented students' suggestions, and in Team C strategies were collaboratively developed by all members. In three teams, initial written reflections and discussion showed team members' ideas were quite well aligned, which eased negotiation processes. However, in Team D, strategy preferences for Paul (teacher) and Piri (student) were quite different, and the team had difficulty reaching agreement.

\subsubsection{Strategies for learning about students' FoK}

\subsubsection{Features of agreed strategies}

Although teams were asked to negotiate and agree at least one strategy by which the teacher could learn about the students' FoK, over the course of two hui all teams chose two or three strategies. Table 9.2 shows selected strategies for each team.

Teams' preferred strategies are sorted into three categories: personal presentation $(P)$, observation of activity (O), and classroom application (C) (Hogg, 2013a). These groupings have been used as the basis for detailed description and discussion of strategies.

Strategies differed in the actions or workload generated for different participants, as well as which participants were involved. Table 9.3 highlights differences between chosen strategies, in terms of workload required of various participants, and settings in which they were conducted. 
Table 9.2: Strategies agreed and implemented by teams

\begin{tabular}{|c|c|c|}
\hline Team & Strategies agreed and implemented by teams & Type \\
\hline \multirow[t]{2}{*}{ A } & Personal presentation by all team members & $\mathrm{P}$ \\
\hline & Co-construction of unit planning & C \\
\hline \multirow[t]{3}{*}{ B } & Teacher observed students in a favorite activity & $\mathrm{O}$ \\
\hline & Student learning about Roman warriors through reflection on Māori warriors & C \\
\hline & Teacher supported student to use self-management FoK in classroom & C \\
\hline \multirow[t]{3}{*}{ C } & Personal presentation by all team members & $\mathrm{P}$ \\
\hline & Students studying Japanese made personal portfolios for students in Japan & C \\
\hline & $\begin{array}{l}\text { Students studying Romeo and Juliet wrote about spouses their families } \\
\text { would not accept }\end{array}$ & C \\
\hline \multirow[t]{2}{*}{$\mathrm{D}$} & Students made a movie about themselves & $\mathrm{P}$ \\
\hline & Students taught the class about a favorite activity & C \\
\hline \multirow[t]{2}{*}{$E$} & Show and tell sessions in a series of TMs & $P$ \\
\hline & Teacher observed students in a favorite activity & 0 \\
\hline
\end{tabular}

Differences between strategies can be summarized in the following list of variables:

- Student autonomy or teacher direction;

- Source: Individual or beyond;

- Process: Individual or collaborative;

- Process: Independent or supported;

- Sources of information;

- Number of opportunities;

- Context: Discrete or integrated;

- Setting: Internal or external to team;

- Workload: Whose? Nature? Level?

- Reciprocity;

- Nature of FoK illuminated. (Hogg, 2013a)

In this section, within each category of strategies, features of teams' chosen strategies are described, with an emphasis on similarities and differences. Also, features of different strategies (see Table 9.3) are discussed and implementation challenges identified. In later sections, implementation challenges are fully described and discussed (Section 9.2.2.2) and implications considered (Section 9.2.2.4) 
Table 9.3: Selected features of chosen strategies

\begin{tabular}{|c|c|c|c|c|c|c|}
\hline \multirow[t]{2}{*}{ Strategy (and team/s) } & \multicolumn{4}{|c|}{$\begin{array}{l}\text { Interpretation of level of workload for participants } \\
\checkmark=\text { light } \checkmark \checkmark=\text { significant } \checkmark \checkmark \checkmark=\text { heavy }\end{array}$} & \multirow[t]{2}{*}{ Setting } & \multirow[t]{2}{*}{ Example of FoK highlighted/ applied } \\
\hline & Students & Teacher & Parents & $\begin{array}{l}\text { Other } \\
\text { students in } \\
\text { class }\end{array}$ & & \\
\hline $\begin{array}{l}\text { Personal presentations by } \\
\text { all team members }(A, C)\end{array}$ & $\checkmark \checkmark$ & $\checkmark \checkmark$ & $\checkmark \checkmark$ & & TM & $\begin{array}{l}\text { Jacob, Team C: family, sports skills, } \\
\text { pets }\end{array}$ \\
\hline $\begin{array}{l}\text { Show and tell sessions at } \\
\text { series of TMs (E) } \\
\text { NB Team E included no } \\
\text { parents }\end{array}$ & $\checkmark \checkmark \checkmark$ & $\checkmark \checkmark \checkmark$ & & & TM & $\begin{array}{l}\text { Shea, Team E: animals, creativity, } \\
\text { family, communication style, care of } \\
\text { environment }\end{array}$ \\
\hline $\begin{array}{l}\text { Observation of favorite } \\
\text { activity }(B, E)\end{array}$ & & $\checkmark \checkmark \checkmark$ & & & Various & $\begin{array}{l}\text { David, Team E: drumming, dancing, } \\
\text { pedagogical FoK, listening skills }\end{array}$ \\
\hline Movie (D) & $\checkmark \checkmark \checkmark$ & & & & & $\begin{array}{l}\text { Peter, Team D: Religious knowledge } \\
\text { and beliefs, drawing, }\end{array}$ \\
\hline Teaching session (D) & $\checkmark \checkmark \checkmark$ & $\checkmark$ & & $\checkmark$ & Classroom & Piri, Team D: online gaming skills \\
\hline $\begin{array}{l}\text { Co-construction of unit plan } \\
\text { (C) }\end{array}$ & $\checkmark \checkmark$ & $\checkmark$ & & & TM & Thor, Team A: sports, health, self-care \\
\hline $\begin{array}{l}\text { Class activity to draw out } \\
\text { students' FoK }(B, C)\end{array}$ & $\checkmark$ & $\checkmark \checkmark \checkmark$ & & $\checkmark$ & Classroom & Briar, Team C: family values \\
\hline $\begin{array}{l}\text { Class activity to support } \\
\text { new content learning }(B, C)\end{array}$ & $\checkmark$ & $\checkmark \checkmark \checkmark$ & & $\checkmark$ & Classroom & Dan, Team B: Māori spiritual practices \\
\hline $\begin{array}{l}\text { Supporting application of } \\
\text { skills in the classroom (B) }\end{array}$ & $\checkmark$ & $\checkmark \checkmark \checkmark$ & & & Classroom & Troy, Team B: perseverance \\
\hline
\end{tabular}




\section{PERSONAL PRESENTATION OF FOK}

Teams A, C, and E implemented strategies which involved team members bringing artefacts or prepared material to a TM and presenting information about themselves, although Team E decided to do this over the course of three TMs. In Team D, the students made a movie about their FoK, which was also a type of personal presentation. All three personal presentation formats potentially gave participants autonomy regarding what to share and how.

Teams $A$ and $C$ generally operated personal presentations like speeches, with each participant taking a turn, thus giving each uninterrupted presentation time. Questions asked afterwards added to information learned. However, an exception occurred when Thor did his presentation. Although he began by relating stories from his babyhood, the dynamic quickly changed to an interview format.

The implementation challenges were:

- Student absent from TM

- Student did not bring artefacts

- Breaking through traditional teacher-student dynamic

Team E's personal presentations were different in several ways: they occurred over a series of TMs, and included conversations and some interview-style interaction (the latter was similar to post-presentation discussion in Teams $A$ and $C$, but longer). Consequently, Team E students engaged in interaction which was more naturalistic (at times), but which did not guarantee each student uninterrupted presentation time.

The implementation challenges were:

- Contributions made by quiet, shy student were infrequent and brief

- Student did not bring artefacts as agreed

- Breaking through traditional teacher-student dynamic

This resulted in David having less speaking time than Shea and Toby, constraining Tom's learning relating to David's FoK.

In Teams A, C, and E, all members gave personal presentations. For Teams A and C, this included parents (Team $E$ included no parents). The rationale for parent presentations was to gather further information to supplement learning from each student's own presentation. Teacher presentations were designed to provide reciprocity and establish mutual sharing (the importance of this for participants is discussed in Section 9.2.3.1). Georgia stated "I know more about the kids because the parents have shared... you know, this is what I do with the kids" (TFG3, lines 74-76).

(There were no implementation challenges). 
Team D students presented personal information within a movie format. The team agreed each student would individually design and make a movie. Paul considered this format would allow the students to demonstrate FoK, and engage in creative and critical thinking. A unique feature of this strategy was the setting in which the information about students' FoK was shared, because Paul viewed the completed work independently. Therefore, there was no opportunity for immediate postpresentation comments or questions.

The implementation challenges were:

- Ongoing delays in completion of the movie

- Students produced a collaborative movie, and two short individual movies

\section{OBSERVATION OF ACTIVITY}

Two Teams ( $\mathrm{B}$ and $\mathrm{E})$ decided the teacher would observe the students in a favorite activity. In all cases the students showed pride and pleasure in the experience, but some observations were more effective for the purpose than others.

Some observations were of students within group settings. A difference between observations was the teacher's familiarity with the group and activity being observed. In Team B, Kate initially observed Dan performing as part of the school kapa haka group. Tom (Team E) observed David at his $\mathrm{Cl}$ performing arts group practice. Tom's observation was his first experience of a $\mathrm{Cl}$ cultural group, which taught him about the group's pedagogical practices, traditional $\mathrm{Cl}$ drumming and dance, and David's drumming and dancing skills.

The implementation challenges were:

- Observation in very familiar setting

- Observation in setting with other students from school also present

Observations of Dan, Troy (Team B), and Shea (Team E) in activities that did not involve other members of the school community were useful for teachers' learning purpose. Kate observed Dan teaching kapa haka to younger students and Troy playing netball as a member of his adult team. Tom observed Shea performing a solo scootering demonstration. All these settings generated new knowledge of students' FoK, without problems arising from other participants in the chosen activity.

Observations involved students in a passive role, simply participating in the selected activity as usual, while also being observed and receiving feedback from the teacher. In all cases, feedback expressed gratitude for the opportunity and appreciation of observed skills. 
The implementation challenge was:

- Individual feedback delayed

Observations took place in various locations and at various times depending on the activity. For example, after school one day, Shea demonstrated his scootering skills at a local school. Troy's netball game was on a Friday evening in a city sports centre. Dan's kapa haka teaching session was in the local area, within school time. Thus, each set of circumstances made different demands on the teacher.

The implementation challenge was:

- Scheduling difficulties

Observation of activity was the second strategy selected by Team E, and members agreed earlier TMs paved the way for this strategy, by establishing trust.

\section{CLASSROOM APPLICATION}

Other strategies sought to bring students' FoK into the classroom in various ways:

- to support learning of new content (Teams B and C);

- to support application of skills in the classroom setting (Team B);

- to draw out students' FoK (Team C);

- to develop a relevant and motivating unit plan (Team A);

- to showcase students' FoK and allow peers to learn from them (Team D).

Some classroom activities scaffolded students' learning by building on their FoK. This approach supported Dan's work in Classics (Team B), Jacob's Japanese vocabulary (Team C), and Briar's understanding of Romeo and Juliet (Team C).

The implementation challenges were:

- Designing a new type of learning activity

- The nature of academic content

- Pressures of school qualifications

- Designing activities which related to FoK and were congruent within teaching and learning programs

Kate (Team B) worked on supporting Troy to use his FoK (including patience and strategic thinking) to manage himself more successfully in the classroom. One aspect was her attempt to gain agreement for Troy's daily report to be personalized.

The implementation challenge was:

- Need for agreement of professional colleagues 
Thor and Sonny Bill (Team A) collaborated with Lizzie to develop a Science unit plan, and some ideas from their meeting were implemented.

The implementation challenge was:

- Timing of implementation

Attempts to showcase students' FoK in classroom settings were less than successful. In response to Tom's suggestion, Team E students declined to teach their classmates about an aspect of their FoK, fearing they would not be respectful. Team D students conducted a teaching session for their classmates, but Peter's fears were realized when a peer said afterwards that it was badly done.

The implementation challenges were:

- Complexity of conducting a teaching session

- Lack of access to equipment

Participants stated their need for a safe environment in which to share personal information, suggesting the importance of settings in which strategies were conducted. All teams that selected personal presentations conducted them within the team environment. As discussed in later sections, the small group setting provided not only safety, but also focused attention and accountability.

\subsubsection{Implementation challenges}

Different strategies had different levels of do-ability for participants, as shown in Table 9.3. Implementing agreed strategies created difficulties and challenges for both teachers and students, affecting valued outcomes achieved. In this section, discussion focuses on challenges experienced by more than one team.

\section{PERSONAL PRESENTATION CHALLENGES}

- Student did not bring artefacts as agreed

- Contributions made by quiet, shy student were infrequent and brief

- Breaking through traditional student-teacher dynamic

In both Teams $A$ and $E$, there was an expectation that participants would bring artefacts to TMs, but neither Thor (Team A) nor David (Team E) did, making learning about their FoK more challenging. Although Thor took charge of his presentation at the beginning, the dynamic shifted and Lizzie took up an interviewer role. From this point, it was evident from silences after he spoke, that Thor took his lead from Lizzie, waiting for her question before speaking. David, a shy student, appeared to arrive at all three TMs without planned topics to talk about, and his contributions were less frequent than other students'. However, in Team E, Tom 
utilized an interviewing format not only with David, but also with Shea and Toby, who brought artefacts and were confident to initiate conversation. Therefore, Lizzie with Thor and Tom with all Team E students relied on interview-like interaction, mirroring traditional teacher-student power dynamics. However, no student expressed discontent with presentation management; all were satisfied with the process. The apparent strength of the traditional teacher-student dynamic is also highlighted by the speech format and largely uninterrupted delivery of Lizzie's and Tom's personal presentations.

When there was an apparent lack of preparedness or fluency in students' personal presentations or show and tell sessions, such as for Thor (Team A) and David (Team E), a range of strategies was used to draw out information. Lizzie relied more heavily on closed questions, mirroring a feature of Tom's approach in TM1. However, in both cases this strategy had limited success, because it tended to generate brief responses. On a number of occasions I observed silences that sometimes occurred in TMs, and searching looks that Tom sometimes gave David. This led me to reflect that Lizzie and Tom seemed to find it challenging to draw out information when students' FoK were quite different to their own, and they were reticent. Early in his TMs, Tom also made extensive use of comments and questions that sought to make links between himself and the students, which was more effective with Toby and Shea than with David, because they shared some of his experiences and interests. Because Tom's team had three TMs, Tom was able to reflect on his practice and adapt his approach. Over three TMs, Tom learned that open questions and allowing students space were more fruitful. Analysis of Thor's presentation confirms that for him as well, open questions were more successful in eliciting elaborated information. In TM3, a series of Tom's questions to David showed a further significant shift. This time, Tom presented himself as completely inexperienced and ignorant of David's Cl cultural practices, and it was from the role of learner that Tom was most successful in eliciting information from David.

\section{OBSERVATION CHALLENGES}

- Observation in setting with other students from school also present

Kate (Team B) initially observed Dan performing in the school kapa haka group. But this was not successful as a learning experience, because Kate had a long history of attending kapa haka performances, and close relationships with many members of the group, which made watching the performance with different intent difficult. The element of challenge due to the familiar setting was unique to Team $B$, but both Teams $B$ and $E$ experienced challenges arising from other students' presence. 
Tom (Team E) also observed David in a setting where other school students were present. Some school students in the $\mathrm{Cl}$ group found Tom's presence to be an invasion of their privacy. However, some others who were uninvolved with the study were pleased to see Tom's interest in the group. Tom reported improvements in his relationships with a parent and another student as a result of the observation.

\section{- Scheduling difficulties}

Multiple responsibilities created scheduling difficulties for Teams B and E. In Team $\mathrm{E}$, a combination of factors resulted in Tom being unable to observe Toby playing soccer as planned - the soccer season ended, and Toby's soccer involvement dropped to participation in a soccer academy, where Tom's observation was not appropriate. Tom's observation of Shea and Kate's observation of Dan (Team B) were both rescheduled a number of times. In the case of Team $E$, this was due to changes in the students' activities, and inclement weather. For Team B, postponements were caused by a clash between the timing of Dan's activity and Kate's teaching responsibilities. At that time of the school year, no funding was available for relief staff, and Kate could not find a colleague who was willing to look after her class, which had a reputation for being challenging. The problem was eventually resolved when I offered to provide relief, and the principal accepted this arrangement because I am a registered teacher.

\section{CLASSROOM APPLICATION CHALLENGES}

- Designing a new type of learning activity

In Table 9.3, I indicated using a class activity to draw out students' FoK involved a high teacher workload, because of the challenges involved in activity design. Lizzie, Kate and Georgia (Teams A, B, and C) all sought meetings with me to get support for their efforts to apply students' FoK to classroom learning. For each of them, working out how they could get students to draw on their FoK to develop new understandings of academic content was a new pedagogical approach. In our meetings, we talked about the current unit of learning and learning objectives, considered students' FoK, and developed ideas for activities. Although teachers initially found this challenging, Georgia and Lizzie quickly started to gain confidence, and Lizzie implemented other classroom activities that related to students' FoK during the course of fieldwork (see Section 9.2.3.4).

\section{- The nature of academic content}

Kate (Team B) wanted to apply students' FoK to their classroom learning, but we could not imagine a way that Troy's FoK could be utilized to scaffold his learning about Greek art and architecture. It was much easier for Kate to plan a way to help Dan learn about the burial rituals related to Roman soldiers; she designed an activity 
where he considered all the relevant aspects of burial for a Māori warrior. It was easy for Dan to complete this first, and it helped him meet assignment requirements.

Similarly, at first Georgia (Team C) could not imagine an activity that would scaffold students' understanding of Romeo and Juliet by drawing on their FoK. Together, we developed the writing task that was implemented.

- Pressures of school qualifications

- Designing activities which related to FoK and were congruent within teaching and learning programs

Teams $B$ and $C$ were affected by time pressure from NCEA requirements and examinations. Students enrolled for NCEA qualifications were Dan (Team B, Level Three), Troy (Team B, Level Two), and Briar (Team C, Level One). Kate and Georgia were determined not to compromise students' success in NCEA. Therefore, they wanted to apply FoK to class learning to support students' NCEA preparation. For Georgia, this caused delays in design and implementation of a class activity, as she firstly prioritized content and skills that were crucial for NCEA. Pressure from NCEA also underpinned Georgia and Kate's focus on developing activities for classroom use that were congruent with the teaching and learning program. This meant that activities implemented by these classes needed to both apply students' FoK and support NCEA achievement.

NCEA pressures affected Team B slightly differently, because Troy and Dan were nearer the end of their school careers. Therefore, supporting their achievement had greater urgency (both Troy and Dan left school at the end of the year in which fieldwork was conducted).

\subsubsection{Time and timing}

The challenge of time and timing warrants separate attention, since it relates to overarching conditions of the study, including fieldwork timing and duration, the time-intensive nature of participation, limited time for teachers to engage with data, relationship-building complexities, and the dynamic nature of FoK.

Due to the lengthy recruitment process, teams began working together in June 2011 , four months after the school year began. This sparked Georgia's concern "I'm a bit worried that given it's Term Three, me getting to know the kids better is a bit tokenistic, you know what I mean? I'm trying to do it in an authentic way that isn't just because" (meeting, 5-8-11). 
The duration of fieldwork, from Hui 1 to Hui 4, varied from 14 to 21 weeks for teams, including four weeks of school holiday time. This was a relatively short timeframe for teams to conduct two cycles of action and reflection, and achieve change.

Teacher participants' workloads were high, and time was a carefully considered resource. Tom (Team E) and Paul (Team D) noted they invested a significant amount of time implementing their teams' agreed strategies. Interestingly, Paul's and Tom's perceptions after the study were both at odds with their expressed ideas beforehand: at recruitment, Paul stated "he did not want to participate if our sessions were going to be too rushed or superficial" (journal, 8-3-11), and Tom indicated that participation would be "a heavy commitment for him, but then quickly added that he was still in" (journal, 8-3-11). By the end of fieldwork, Paul judged benefits to be insignificant and not worthy of the time. However, Tom consciously invested time to ensure his students felt valued. Although he estimated he spent 15 hours implementing three TMs, three observations, and a team celebration, as well as team hui to decide and evaluate strategies, Tom considered this time well spent. Scarce time also led to limited engagement with videotaped information regarding students' FoK by all teacher participants (see Section 10.2.1).

Pearl and Georgia (Team C) considered that, as for the development of other relationships, building knowledge of students' FoK would necessarily take time, as trust is developed and participants become comfortable to share more private information. Team E's selected strategies went some way towards achieving this, by providing multiple TMs over a period of five weeks. Team E students confirmed their growing closeness with Tom increased their comfort with him, leading to agreement to be observed in an activity. An additional feature of multiple opportunities was allowance for sharing information previously left out. For example, Thor (Team A) stated he would have valued the opportunity for a second personal presentation, to add information he forgot. Pearl (Team C) and Ses (Team A) also noted that students' FoK were dynamic, so therefore multiple opportunities would allow a teacher to update knowledge.

\subsubsection{Choosing strategies to suit participants and settings}

Descriptions of strategies and challenges experienced in their implementation highlight the relevance of various skills for their successful completion. Therefore, participants' skills affected do-ability and effectiveness of strategies.

Verbal skills were required for a number of strategies implemented. Personal presentations called for verbal skills, as participants delivered speeches, sometimes with the aid of artefacts. All teams implementing personal presentations used turntaking to methodically give all members an opportunity for receiving focused 
attention. At the beginning of TM1, Tom stated "we'll talk a little bit, and then we'll pass on to the next person, 'cos otherwise, we don't want anyone to have too much time" (0m.00s-0m.08s). Some students, such as David and Toby (Team E) and Sonny Bill (Team A), were very quiet during presentations by others. However, Toby's confidence, verbal proficiency and assertiveness resulted in more contributions than Shea and David in Team E's TMs. Also, at times his friend Shea seemingly went into conversational mode during Toby's presentations, making frequent contributions. Therefore, strategies that required students to verbalize FoK had differing levels of difficulty. When delivering personal presentations, turn-taking reduced risk of losing teacher attention, but when they were managed as interviews, this strategy was more demanding. The level of difficulty of students conducting teaching sessions in class was even higher, because of the additional risk of losing peers' attention, with specific skills being required to convey material effectively.

In summary, strategies that relied on verbal delivery by students worked well for individuals who were confident and articulate, and presented elaborated information.

Some students chose to use other communication skills as well when doing personal presentations, show and tell sessions, or teaching sessions. Furthermore, when this occurred, both the effectiveness of communication and learning about FoK tended to increase. A number of students demonstrated skills with information technology. For example, Toby designed an animated powerpoint, Shea used cellphone operational skills to show photos, and Briar connected her family video camera to a television to show video footage. These skills were used to enhance presentation of information, and their application inherently gave information about FoK. In all three examples above, the students used equipment from home. Therefore, access to equipment affected students' choice of delivery tools.

Access to appropriate equipment also influenced success of strategies in highlighting students' FoK. For example, Piri and Peter's teaching session about an online game was delivered without access to a computer or any other material (e.g., printed screenshots), constraining its effectiveness.

Preparation of a movie about one's own FoK also potentially involved verbal skills, as well as audio and video recording operational skills.

In several teams, participants who knew each other well actively supported one other to teach teachers about their FoK. This proved to be an effective way to increase the breadth and depth of information shared. Given New Zealanders' cultural distaste for showing off, students providing information about each other was a culturally appropriate way to make statements about a person's areas of expertise. 
Students supported each other in show and tell sessions (Team E), when Toby and Shea prompted each other to talk about topics, and Shea contributed to Toby's storytelling, highlighting their shared experiences. Peter and Piri's (Team D) collaboration was their independent decision; prior to this, they had not completed individual movies as agreed. In their collaborative movie, Peter and Piri talked about themselves and described each other's FoK. In this case, each student described and praised each other's strengths and expressed faith in each other's potential, while the other listened and murmured self-deprecating remarks.

Ses (Team A) also contributed information about his step-son Sonny Bill by interjecting comments and prompting him to discuss or elaborate on topics.

\subsubsection{Participants' satisfaction with agreed strategies}

Participants reported satisfaction with agreed and implemented strategies. However, Team $C$ was the only team that decided not to implement additional strategies at their second hui. Three teams made refinements to strategies, and four teams chose further strategies after completing their original choice. Therefore, data suggest satisfaction with the team process (Hogg, 2012) may have affected satisfaction reported for selected strategies. A further factor that may have influenced participants' perceptions could be a focus on strategies as implemented.

\subsubsection{Significance of applying strategies that are agreed}

In this study, the teams' role was collaboration to decide strategies for implementation. Thus, the research design attempted to respect multiple participants' voices. Therefore, I argue the notion of agreement regarding strategies was important, since it was expressed as fundamental in the establishment of fieldwork. Findings discussed earlier highlight diverse strategies selected by teams, and those not selected, such as home visits and community learning projects. Furthermore, individual participants' reflections show no participant preferred home visits.

Findings also highlight the significance of agreement (Hogg, 2013a). Seeking and honouring agreement was seen as demonstration of valuing all participants. Also, reaching agreement within teams was perceived to enhance all members' ownership and commitment. Team $\mathrm{D}$, in which agreement about strategies was problematic, was the one team in which implementation was fraught and did not result in significant learning about students' FoK, according to all team members. Lastly, strategies conducted in other settings raised questions about consequences of agreement for others outside teams, such as other members of the $\mathrm{Cl}$ performing arts group. 


\subsubsection{Valued outcomes}

This section addresses four main valued outcomes. For each, links are made to participants' hopes, to illuminate the value assigned to that outcome by participants across teams. Also, evidence is presented to highlight commonalities and differences in teams' achieved outcomes.

\subsubsection{Learning about students' FoK}

The hope that teachers would get to know students better was expressed by four parents, two students, and two teachers. Tash and Catherine discussed the importance of teachers not making assumptions about students. Sandy agreed it was desirable for students to be more than "just a face in the classroom" (Hui 2, line 212). Kate reported related feedback from KHS students in a recent survey: "the kids said can you know us as individuals please" (Hui 4, line 396). She felt that it was important for her to know "what aspects/ funds of knowledge have occurred in (my student's) life to bring him to become the student/learner who is in front of my class" (post-hui 1 reflection). Briar stated "in a perfect world... the class will know something about the teacher and the teacher would know something about the students as more than just like... their school work" (interview, lines 93-95).

Findings related to teachers' new knowledge of students' FoKs were mixed. All teachers reported learning new information about their students' FoK, regardless of prior relationships and knowledge. Kate and Dan (Team B) agreed Kate learned new information about Dan's FoK, although she knew him very well previously. Kate considered new knowledge resulted from her focus on Dan as an individual. For Tom, the learning about students' FoK revealed unimagined aspects of their lives. He stated "the horse blew me away. The fact that he's got a horse in his back paddock. I just never would have thought of Shea riding horses, which is a really posh thing where I come from" (TFG1, 1h.10m. 57s-1h.11m.08s).

Table 9.3 outlines some examples of FoK demonstrated through each strategy. Therefore, data from this study confirm other findings that a range of strategies may be utilized to learn about students' FoK (see Table 9.2).

Perceptions of the depth and breadth of teachers' knowledge of students' FoK varied. Georgia (Team C) considered she "learnt lots" (TFG3, line 220) about her Year 9 Japanese class, Year 11 English class, as well as about students in the team. She stated personal presentations by parents were helpful because they gave her a "fuller picture" (Hui 3, line 85) of Briar and Jacob. However, other teachers were more tentative in their descriptions of knowledge gained. Lizzie (Team A) described a range of Thor's and Sonny Bill's FoK, but considered she still had a lot to learn about their 
strengths and skills. Tom (Team E) stated he was left with a series of "enduring images" (interview, line 113) relating to the students, representing key concepts or metaphors regarding their FoK. Interestingly, this approach to understanding the students privileged an holistic understanding, and did not incorporate detail.

Generally, parents and students appeared to be more confident about teachers' knowledge gains. For instance, Sandy (Team C) considered Georgia gained understanding of a "totally different side to the person" (PFG3, line 989). Ses (Team A) was confident Lizzie gained a good understanding about their family beliefs and priorities. Team B parents agreed that Kate "got to know (Troy) really well" (Catherine, PFG3, line 277). Jacob (Team C) was satisfied that Georgia would have gained a full picture of him from his presentation. Team E students considered Tom gained knowledge about their interests, personalities, family heritage and activities, and "cultural things" (Shea, interview, line 34). Data do not provide an explanation of differences in perceptions between teachers and other participants. However, I wonder whether differences relate to students' and parents' perceptions arising from information shared, in contrast with teachers' perceptions arising from information learned. I argue that learning about FoK is complex because it involves teachers in a new approach to looking at students. As for other learning, simply hearing or seeing new content once does not mean it is learned. As noted earlier, González et al.'s (2005) recommendation that teachers learning about their students' FoK participate in collective viewing and discussion of data, highlights the challenge of this work, and enhancements to understanding achievable through prolonged reflection on data. Therefore, although significant data about FoK was shared, the teachers may not have maximized their learning.

In contrast with findings from other teams, Paul (Team D) noted Piri's and Peter's skills as a comedy double act, but learnt nothing else about them. Piri and Peter also expected Paul had not learned much about their FoK. Paul said he would have valued learning more about challenges the boys faced at home, but this knowledge did not arise from strategies implemented. He considered the insubstantial knowledge gained meant implementation of strategies was not worthwhile.

In Teams A and C, teachers' new knowledge of students' FoK was enhanced by knowledge of parents' FoK. In both these teams, parents participated in personal presentations. However, there were different perspectives about inclusion of parent participants as a source of learning for teachers. Thor (Team A) felt Lizzie would have learnt more about his FoK if another family member had been involved. Lizzie considered parents would have the knowledge and pride to share their child's FoK, thus usefully complementing a student's self-reporting. Nevertheless, she did not 
believe she had learnt much about Sonny Bill from his step-father Ses, and concluded changes in the strategy set-up could have changed this outcome. However, students from Teams $D$ and $E$ reported they would have been less open if parents were present. Shea's (Team E) concern was parents would share embarrassing and irrelevant information about their distant babyhood and childhood. Peter and Piri (Team D) and Shea and Toby (Team E) provided information related to each other's FoK, highlighting other possible sources of information for teachers.

After personal presentations by all members, Lizzie (Team A) reported her enhanced appreciation of individuals' complexity, but stated it was difficult to identify students' strengths and skills.

Data from teacher participants highlighted their lack of time to review video-footage related to students' FoK, possibly constraining their learning about students' FoK. Within TFG1 and TFG2, teachers considered readings and video-footage extracts from TMs. All agreed collaborative reviewing and reflection was very worthwhile, confirming the important "mediating functions" (González et al., 2005, p. 18) of such a forum for teacher participants. Unfortunately, time in TFGs did not allow review of all data. After TMs, I provided a copy of the video-footage to the teacher and encouraged them to review it. However, all teachers except Kate stated they had not had time to do so. Therefore, I wonder how the depth of teachers' understanding of students' FoK could have been enhanced by greater engagement with videofootage.

Parent and student participants in Teams A, C, and E also reported new learning about the teacher, which they all valued highly. Pearl rated this as more important than teachers learning about students' FoK, stating "I actually think it was actually learning about the teacher's FoK that actually helped" (Hui 3, lines 199-200). Thor (Team A), David, Shea, and Toby (Team E) agreed that valued outcomes were achieved because "I know (the teacher) better" (David, interview, line 610). Various reasons were offered to explain the importance of learning about the teacher. Sandy (Team C) and Toby (Team E) stated that it was important for teachers to reciprocate by sharing about themselves, because to expect others to share without reciprocation would not have been appropriate. Tash (Team B), Toby (Team E), Briar, and Jacob (Team C) agreed that gaining mutual understanding of each other was important. Thor and Sonny Bill (Team A), and Briar (Team C) stated learning about their teachers enabled them to see them as more than a teacher, helping them to connect with them as people. 


\subsubsection{Impacts on teacher-student relationships}

Six students, two parents, and two teachers expressed hopes for improved teacherstudent relationships. A number of students hoped to find a way to "connect with the teacher" (Shea, post-hui 1 reflection). Troy, however, hoped for respect. Tash and Catherine believed that effective teacher-student relationships were "not just a straight teacher-student relationship sort of thing" (Hui 4, line 406), but broke through traditional notions of professional distance. For Catherine, this was how "the teacher makes the student feel worth something" (interview, lines 67-68). Tom also hoped for closer relationships with students, as they developed the secure knowledge that their teachers valued them. He believed this would lead to a general improvement in learning behaviors. However, Lizzie hoped that closer relationships with families would help her to design more relevant learning experiences.

Evidence from teachers, students and parents confirmed that as teacher and student participants grew to know each other better, they developed closer relationships. Although data suggest that benefits occurred in other relationships as well (e.g., teacher-parent), I will focus on benefits to teacher-student relationships.

Most student participants reported their relationship with the teacher had improved, but to varying degrees. Before and after fieldwork students completed a rating scale item in which they chose the statement which best described their relationship with the teacher: (1) Very good; (2) Mostly good; (3) Sometimes difficult; (4) Difficult; (5) Too early to tell; (6) I don't have a relationship with this teacher. Students' responses generally indicated improvement in student-teacher relationships over the course of fieldwork, as shown in Table 9.4. The exceptions were Briar (Team C) and Dan (Team B), who reported their relationships with Georgia and Kate respectively were consistently very good, and Peter (Team D), who reported that his relationship with Paul remained sometimes difficult.

Table 9.4: Students' assessment of relationship with teacher before and after fieldwork

\begin{tabular}{lccccccccccc}
\hline $\begin{array}{l}\text { Reported } \\
\text { relationship }\end{array}$ & $\begin{array}{c}\text { Sonny } \\
\text { Bill }\end{array}$ & Thor & Dan & Troy & Briar & Jacob & Peter & Piri & David & Shea & Toby \\
\hline Before & 2 & 3 & 1 & 2 & 1 & 1 & 3 & 3 & 3 & 2 & 2 \\
After & 1 & $\begin{array}{r}\text { Better } \\
\text { than 1 }\end{array}$ & 1 & 1 & 1 & $\begin{array}{r}\text { Better } \\
\text { than 1 }\end{array}$ & & & & &
\end{tabular}

Changes in students' behaviors also evidenced improved student-teacher relationships. Troy (Team B) began to demonstrate trust in Kate's understanding of him, by seeking her out when he needed space, and Dan began to seek Kate's support for a wider variety of issues than previously. Kate, who stated that she 
"stalked" students to follow up issues, noted she no longer sought out either Troy or Dan, because they came to her. Briar (Team C) also began to seek support from Georgia: "whereas she probably wouldn't have beforehand... she felt more comfortable with Georgia for some guidance" (Sandy, interview, lines 126-128). Similarly, Shea, Toby, and David (Team E) routinely sought Tom's support whenever any one of them was in trouble. Thor (Team A) showed Lizzie family photos which he withheld from classmates. Sonny Bill (Team A) stated that, knowing Lizzie well, he would greet her out of school, which was beyond his practice with other teachers.

Data from all teams (including statements made by participants and reported changes in behavior) indicate participants believed that teachers changed their perceptions and treatment of students as a result of their new learning relating to students' FoK. Thor (Team A) and Jacob (Team C) both believed their teacher now understood their inherent goodness, and would not over-react to any isolated incidents of inappropriate behavior that might occur in class. During the course of fieldwork, Jacob was suspended as a result of an incident in another teacher's class. Both he and Pearl considered that had the incident occurred in Georgia's class, she would have managed the situation differently and he would not have been suspended. The boys in Team E agreed Tom was "softer" (Toby, interview, line 195) and "not as grouchy" (Shea, interview, line 201), which they attributed to his enhanced knowledge of them and tolerance of their ways of being (such as their love of joking). Peter and Piri (Team D) also considered Paul became more tolerant of their joking manner in class, and noted he now gave them easier work.

The new teacher-student relationships were described by participants in Teams $A$, $B$, and $E$ as breaking through traditional student-teacher roles. However, it is unclear whether this change extended into classrooms.

Due to findings that teacher knowledge of students' FoK improved teacher-student relationships, all participants agreed teacher learning about students' FoK would be valuable early in the school year. Furthermore, they agreed it would be most desirable to begin teachers' learning of students' FoK early in their time at the school.

\subsubsection{Effective learning behaviors}

For the purpose of this study, I categorized certain impacts on students as effective learning behaviors. These include in-class behaviors such as attendance, increased verbal participation, perseverance, engagement, prompt refocusing, enjoyment, and increased work output. Also, seeking additional support from the teacher is categorized as an effective learning behavior, although this occurs outside 
classroom settings. These behaviors have also been identified by literature (such as Riley, Robinson, \& Elliott, 1993). (see also Section 9.2.3.2.)

Parents expressed the hope that spending time with students to learn about their FoK would improve their effective learning behaviors. For instance, Catherine and Tash agreed that by "trying to see what makes these kids tick" (Catherine, interview, line 148), they hoped students would become "willing to take more focus" (post-Hui 1 reflection). Ses hoped it would "keep them motivated for their future" (post-Hui 1 reflection). Pearl stated:

For me, it's about engagement of Jacob in lessons, and l'm hoping that, if the teacher knows more about him, is able to use that knowledge in lessons to engage him, or keep him from getting distracted, maybe able to keep him in line... as a result he'll be more focused or he will be more engaged, and thus there'll be more learning taking place, and as a result of the learning, higher achievement. That's what I'm hoping. (post-Hui 1 reflection)

This theory is supported by Jacob's words:

There's more respect that goes into your work and stuff you work on. You work extra harder... and you just like be more respectful than you normally are, instead of just doing the work. (Team C Hui 3, lines 324-326)

These words suggest when teachers show more commitment towards students, students reciprocate with greater effort. I argue this principle is also demonstrated by changes in Dan and Troy's interaction with Kate; by seeking more frequent support from Kate they were reciprocating her commitment and efforts towards their learning with greater commitment and effort towards their own learning.

Tom saw learning about students' FoK as "an investment approach, which enables both parties to open their level of interest" (interview, lines 189-190). He stated "This definitely affects their willingness to do the hard stuff" (post-TFG1 reflection). In PFG1, parents theorized about the importance of rapport, and impacts of the nature of teacher-student interaction on learning behaviors. They agreed that there was a direct relationship between how well teachers and students knew each other and the loyalty, commitment, and effort that teachers and students gave each other.

As predicted by the parents, students in Teams $A$ and $E$ experienced changes in their general level of effective learning behaviors as a result of the study. This was particularly pronounced for Thor (Team A), whose attendance and participation prior to fieldwork had been causing concern. After the study, both Thor and Lizzie confirmed his attendance had become reliable. Thor's engagement in class also improved significantly; he became actively involved and began to express enjoyment of school. Sonny Bill (Team A), Shea, Toby, and David (Team E), whose attendance was reliable 
before the study, also demonstrated improved learning behaviors. All three showed greater active involvement in their learning. For instance, Sonny Bill asked more questions and refocused more quickly after distractions. Shea and David both increased their work output, and David began to contribute verbally in class. Thor, Jacob, Shea, Toby, and David all showed improved perseverance.

Students in Team C, and their classmates who participated in class activities which related their FoK to learning, also benefited from improved engagement. However, in this case, Georgia and the students agreed the activities for both classes were highly motivating, generating improved engagement levels for the work associated with the activity. I argue participation in both activities was seen as generating real benefits for students. By drawing on their FoK, one strategy provided a way for students to develop international links, and the other promoted reflection on a topic that students valued. Therefore, drawing on students' FoK in class activities motivated students by enhancing task authenticity (Fox-Turnbull, 2012).

\subsubsection{Applying FoK to classroom pedagogy}

Implementation of strategies that linked students' FoK to classroom learning was undertaken by three teams ( $A, B$, and $C)$. For all teachers involved, these were new developments in their pedagogical practice. Findings related to the application of students' FoK to classroom practice were also reported in Section 9.2.2.1, as these are simultaneously strategies which teams agreed to implement, and outcomes which were valued, as defined by participants' hopes.

All groups of participants expressed hopes related to applying FoK in class to support learning. Statements often highlighted links drawn by participants between engagement, learning, and pedagogical approaches. Students hoped that knowing their FoK would mean teachers "stop boring us" (Shea, interview, line 145) and help them to "understand us better and they can put stuff that we like into our work to make us do good work" (Toby, interview, lines 62-63). Thor also hoped for "better work, more fun work" (post-hui 1 reflection). Students hoped that "respect for the way that I want to learn" (Troy, post-hui 1 reflection) would result in "cater(ing) the lessons to the students' needs" (Briar, interview, lines 109-110). For Shea, this hope included "giv(ing) us work that he knows we can do, like don't just give us baby work, give us more work" (interview, lines 72-73). Similarly, parents hoped that knowing "this is the way they think, this is the way (teachers) could actually teach it to grasp their young minds" (Tash, interview, line 423). Catherine also hoped that teachers would "put that funds of knowledge to use in the classroom and that would make the kid feel super special" (interview, lines 138-138). All teacher participants 
shared the hope that "using students' funds of knowledge, I can make learning richer and more relevant" (Georgia, post-TFG1 reflection).

As stated in Section 9.2.2.1, the nature of classroom applications varied across teams, and therefore cross-team analysis of findings is necessarily limited. However, changes in teacher practice during fieldwork are summarized in Table 9.5.

Table 9.5: Application of FoK to classroom pedagogy by teams

\begin{tabular}{llllll}
\hline Strategy: & A & B & C & D & E \\
\hline Support learning of new content & $\checkmark$ & $\checkmark$ & $\checkmark$ & \\
Support application of skills in classroom setting & & $\checkmark$ & & \\
Draw out student FoK & $\checkmark$ & & $\checkmark$ & \\
Co-construct unit plan & $\checkmark$ & & & \\
Showcase students' FoK so peers can learn from them & & & & & \\
\hline
\end{tabular}

$\checkmark$ Implemented, as per team agreement

$\checkmark$ Implemented, additional to team agreement

The table highlights further changes to practice implemented by Lizzie (Team A), reflecting her growing confidence and belief in the value of pedagogical approaches that relate to students' FoK. Also, teachers expressed intentions to make future changes to their practice, such as development of relationships with parents (Kate, Team B) and revision of planning templates (Georgia, Team C). Intended next steps were developed as a result of reflection on valued outcomes achieved.

Reflection on data evidencing valued outcomes in relation to participants' hopes highlights achievements in some teams. Four teachers implemented new pedagogical approaches which sought to offer higher levels of personal interest and relevance for students, through relating learning to FoK. A further area for development which appears not to have been achieved within this study arises from Shea's hope; data do not suggest that, having learnt about their FoK, teachers raised the academic level of work given to students.

\subsection{Conducting the TBC approach}

Findings related to implementation of the TBC methodology highlight challenges and elements that supported fieldwork to run smoothly.

\subsubsection{Challenges}

\subsubsection{Recruitment and retention of students and parents}

During fieldwork, it became apparent that gaining and maintaining parent participants were the biggest challenges to teams. Recruitment of teams in which participants 
shared an authentic connection was lengthy, and I needed patience and persistence to successfully establish the teams. Although recruitment of teachers was relatively straightforward, recruiting student and parent participants was more challenging.

Many parents had heavy family responsibilities, including care of other children and elderly parents, which influenced their decisions to decline to participate (Catsambis, 2001). Three teams were affected by these circumstances.

To honour the principle of he kanohi kitea ${ }^{38}$. I offered several opportunities for potential participants to learn about me and the study. I ran five recruitment meetings for students and held meetings with family members at times and places to suit them. Patience was required, as meetings did not always go ahead as planned: four meetings did not happen due to sickness, and twice I arrived at a family home to find no-one there.

The importance of he kanohi kitea was borne out by my observations that all but one potential student participant met with me more than once before making their decision, and one parent wanted to meet more than once before making their decision. Nevertheless, three parents gave consent for their children to participate without meeting me face-to-face.

\subsubsection{Finding my role}

\section{PARTICIPANT OBSERVATION}

Approaching this study as a beginning researcher, I planned to facilitate team events to support expression of all participants' voices, and imagined myself as an impassive observer. However, in the field this vision felt inappropriate according to my understanding of how to behave in small groups, particularly due to the personal nature of interaction. I resolved my dilemma of how to be with teams by becoming a participant observer.

According to Denzin (1989), participant observation is "a field strategy that simultaneously combines document analysis, interviewing of respondents and informants, direct participation, and observation, and introspection" (pp. 157-158). It involves observing as a member, and influencing what is observed by participating. Therefore, a challenge became enacting participant observation and also maintaining distance. To maintain trustworthiness, I needed to be rigorous in my reflexive processes, and to always look for evidence when interpreting data.

Flick (2006) notes researchers must identify "observational situations in which the problem under study becomes really 'visible' “ (p. 222). These can be described

\footnotetext{
${ }^{38}$ Face-to-face discussion
} 
using nine dimensions (Spradley, 1980). Of these, observation situations I selected were situations and places in which team members met as a group in order to conduct team activities (e.g., negotiating strategies). My records included data relating to physical objects which played a role in these events, participants' actions, and feelings. I collected data about participants' goals (hoped-for valued outcomes). Although teams were constructed for the purpose of the study, I would argue that observation of team processes, actions, and outcomes was relevant because construction of teams was centered around people with authentic relationships.

Jorgensen (1989) identifies seven features of participant observation, considered in Table 9.6 in relation to the present study. Thus, Table 9.6 demonstrates how I enacted the role of participant observer.

As fieldwork progressed, participants affirmed my decision. Thor (Team A) commented "in big teams they have different people talking and you were just sitting there like, yes, all I need to do really" (interview, lines 540-541), highlighting the impossibility of an individual being unnoticed in a small team. Several teacher participants commented on the impact of my approach. Kate (Team B) stated:

I needed that, that's what I wanted, I didn't want to do this all on my own. And you could tell just that you cared just as much about what my boys were doing as I did and their parents did. Yeah I knew even though yes you were the facilitator and etc you were that role I always felt that you cared just as much about those boys as well. (interview, lines 890-894)

\section{Table 9.6: Features of participant observation related to this study}

\begin{tabular}{ll}
$\begin{array}{l}\text { Features (from Jorgensen, } \\
\text { 1989, pp. 13-14) }\end{array}$ & \multicolumn{1}{c}{ In the present study } \\
\hline $\begin{array}{l}\text { Interest in meaning and } \\
\text { interaction from } \\
\text { members'/insiders' } \\
\text { perspective }\end{array}$ & $\begin{array}{l}\text { What were participants' perceptions of valued outcomes? How did } \\
\text { participants experience team processes/ interaction? How did } \\
\text { teachers interpret video footage relating to students' FoK? How did } \\
\text { teachers interpret knowledge about students' FoK for their practice? }\end{array}$ \\
$\begin{array}{l}\text { Everyday life situations and } \\
\text { settings }\end{array}$ & $\begin{array}{l}\text { Observation of team interaction, including all hui and other team } \\
\text { events (excluding teacher-student interaction in classroom settings, } \\
\text { except for team events in classroom settings). }\end{array}$ \\
$\begin{array}{l}\text { Emphasis on interpretation } \\
\text { and understanding of human } \\
\text { existence }\end{array}$ & $\begin{array}{l}\text { What was the nature of interactions that took place? How did these } \\
\text { affect valued outcomes achieved? Did teams collaboratively agree on } \\
\text { strategies? What was the meaning of things that happened? Why did }\end{array}$ \\
$\begin{array}{l}\text { Open-ended, flexible, } \\
\text { they happen? } \\
\text { focus redefined depending } \\
\text { on data gathered }\end{array}$ & $\begin{array}{l}\text { Teams negotiated strategies for implementation. Teachers chose } \\
\text { students to invite, with limited criteria imposed. Participants defined } \\
\text { the unit of analysis: valued outcomes. }\end{array}$ \\
$\begin{array}{l}\text { In-depth, qualitative case } \\
\text { study approach/design }\end{array}$ & \begin{tabular}{l} 
Collective case study, consisting of five cases. \\
\hline
\end{tabular}
\end{tabular}


Importance of relationships with those in the field

Direct observation coupled with other data collection methods
Due to small team size, it was unrealistic to consider participants could be unaware of my presence. I took a facilitation role in teams, to promote and support student voice, anticipating they would be least confident and least powerful in the process; therefore, I needed to gain all team members' trust. Personal information was shared in teams, and remaining neutral when this happened would have been culturally inappropriate and rude.

I attended and recorded all TMs and team events. Data were also collected in questionnaires, photos, FGs and individual interviews. I collected data about my own role in processes and outcomes.

Teams $\mathrm{A}$ and $\mathrm{C}$ also requested me to participate in their agreed strategy by giving a personal presentation about my FoK, indicating that within the intimate team setting, reciprocity was important. I found this request challenging. On the one hand I had become a participant observer in teams, and it seemed inappropriate for some individuals to share information while I simply observed. As well, I was aware my relationships with participants influenced the success of this study, and sharing information could enhance trust. On the other hand, relationships between teachers, students, and parents were the authentic relationships upon which teams were built, and I did not wish to attract attention from that grouping to myself. My view was that I was the least important person in the mix. Therefore, my response was to explain my ambivalent thoughts, and ask if I could share my profile at the teams' final meeting (Hui 4). However, I struggled to lose a feeling that I let the teams down with my response.

\section{RESEARCHER CONTRIBUTIONS}

According to the research plan, I facilitated team events to support all members to express themselves. However, this task required more input than I anticipated. Table 9.7 is an analysis of my own contributions to team hui for Teams $A$ and $E$, in order to identify the nature and impact of my presence. Numerical data in Table 9.7 refer to the quantity of my verbal contributions. Although further analysis to quantify words spoken would be valuable, time constraints prevented this.

Table 9.7 presents all my verbal contributions to team hui sorted into 17 categories. There were variations in the quantity of verbalizations in different team hui, as each event was affected by different situations. For instance, lateness affected Team A's Hui 2 and Hui 3, leading me to repeat some information. Thor's mother had not attended Hui 1, and in Hui 2 I answered her questions about the research process. In a further example, Team E's Hui 2 included lengthy organizational discussion, as we explored options for Tom to observe the students. 


\section{Table 9.7: Researcher contributions in team hui}

\begin{tabular}{|c|c|c|c|c|c|c|c|c|c|}
\hline \multirow{2}{*}{ Focus } & \multirow{2}{*}{ Nodes } & \multirow{2}{*}{ Definition } & \multirow[t]{2}{*}{ Total } & \multicolumn{3}{|c|}{ Team A } & \multicolumn{3}{|c|}{ Team E } \\
\hline & & & & Hui 2 & Hui 3 & Hui 4 & Hui 2 & Hui 3 & Hui 4 \\
\hline \multirow[t]{8}{*}{ Facilitation } & $\begin{array}{l}\text { Acknowledging } \\
\text { responses }\end{array}$ & $\begin{array}{l}\text { Items which show active listening, including } \\
\text { fillers e.g., mmm, okay }\end{array}$ & 106 & 57 & 20 & 11 & 3 & 10 & 5 \\
\hline & Agreement & Stating what has been agreed, and related items & 17 & 6 & 3 & 0 & 3 & 5 & 0 \\
\hline & Asking for ideas & $\begin{array}{l}\text { Asking participants questions, including asking for } \\
\text { their opinion, experience, suggestions, elaboration }\end{array}$ & 168 & 19 & 29 & 45 & 15 & 31 & 29 \\
\hline & $\begin{array}{l}\text { Explaining procedure } \\
\text { of current hui }\end{array}$ & $\begin{array}{l}\text { Items about the procedure for conducting the } \\
\text { current research hui }\end{array}$ & 30 & 12 & 7 & 1 & 2 & 8 & 0 \\
\hline & $\begin{array}{l}\text { Explaining purpose of } \\
\text { current hui }\end{array}$ & Items relating to the purpose of the current hui & 27 & 4 & 1 & 5 & 2 & 7 & 8 \\
\hline & Latecomers & $\begin{array}{l}\text { Items relating to participants who arrive late to } \\
\text { the current hui }\end{array}$ & 18 & 8 & 10 & 0 & 0 & 0 & 0 \\
\hline & Student management & $\begin{array}{l}\text { Items which call for attention, regain focus, or } \\
\text { change students' behavior }\end{array}$ & 15 & 1 & 0 & 0 & 0 & 11 & 3 \\
\hline & $\begin{array}{l}\text { Summarising, } \\
\text { paraphrasing }\end{array}$ & $\begin{array}{l}\text { Items in which I summarise what has been said } \\
\text { by the previous speaker(s) }\end{array}$ & 72 & 17 & 14 & 13 & 4 & 17 & 7 \\
\hline \multirow[t]{2}{*}{ Checking meaning } & Asking for information & $\begin{array}{l}\text { Asking questions to clarify something with which } \\
\text { I'm unfamiliar }\end{array}$ & 13 & 4 & 5 & 1 & 0 & 3 & 0 \\
\hline & $\begin{array}{l}\text { Checking my } \\
\text { impression }\end{array}$ & $\begin{array}{l}\text { Items which refer to actions or statements from } \\
\text { previous occasions, or checking understanding } \\
\text { of current action or statement }\end{array}$ & 14 & 6 & 0 & 7 & 0 & 1 & 0 \\
\hline \multirow[t]{4}{*}{ Research process } & $\begin{array}{l}\text { Answering questions } \\
\text { about the study }\end{array}$ & $\begin{array}{l}\text { Answering questions about the study, but } \\
\text { unrelated to the current hui }\end{array}$ & 26 & 19 & 5 & 0 & 0 & 2 & 0 \\
\hline & $\begin{array}{l}\text { Organising and } \\
\text { admin }\end{array}$ & $\begin{array}{l}\text { Items relating to future events in the study which } \\
\text { have been agreed }\end{array}$ & 73 & 16 & 10 & 5 & 1 & 30 & 11 \\
\hline & Stating constraints & $\begin{array}{l}\text { Items relating to things that are not possible } \\
\text { within the study }\end{array}$ & 5 & 0 & 1 & 0 & 1 & 2 & 1 \\
\hline & $\begin{array}{l}\text { Comments from my } \\
\text { FoK }\end{array}$ & $\begin{array}{l}\text { e.g., from my teacher identity, or from living in } \\
\text { the Cook Islands }\end{array}$ & 8 & 3 & 0 & 0 & 2 & 2 & 1 \\
\hline Manaakitanga & Catering & Items which relate to the food or drink provided & 26 & 8 & 10 & 0 & 0 & 8 & 0 \\
\hline \multirow[t]{2}{*}{$\begin{array}{l}\text { Participant } \\
\text { observation }\end{array}$} & Personal remarks & $\begin{array}{l}\text { Comments or questions regarding an individual's } \\
\text { action or achievement }\end{array}$ & 43 & 10 & 8 & 2 & 0 & 12 & 11 \\
\hline & Suggestions & Items in which I put forward an idea & 32 & 10 & 9 & 1 & 5 & 7 & 0 \\
\hline
\end{tabular}


Occasionally I found student actions caused distractions, and drew on my teaching identity to address the matter. Team E's Hui 3 included a number of student management items, such as:

Okay, are you ready to concentrate? 'Cos we've probably only got till $4 p m$, have we? (line 376)

I frequently asked for ideas, often directing questions at specific individuals, seeking a range of perspectives. When participants made suggestions, I sought elaboration to hone in on the detail of what a suggestion might look like. Conversation was more fluent in Hui 2, as participants discussed ideas about possible strategies to implement. However in Hui 3 and 4, it seems that my facilitation was more intrusive. A possible explanation is I guided conversation to gather data related to a range of factors (e.g., do-ability and effectiveness in generating learning about FoK). A further possibility was that participants were less confident to critically evaluate implemented strategies without guided facilitation.

When I made suggestions, these usually related to ideas for strategies previously suggested or agreed. For example, I suggested that Team A students prepare to coconstruct the unit plan with Lizzie by talking at home about it. I also made suggestions about the time between implementation of strategies and the next team hui (when impacts would be discussed).

Participants also valued attempts to accommodate diverse needs in teams. During hui, I observed individuals during reflection, discussion and negotiation processes, and endeavoured to facilitate hui to meet their preferences and needs. For instance, Briar (Team C) commented "I sometimes have trouble putting my thoughts into words exactly the way I want them" (interview, lines 218-219); therefore, Team C hui began with quiet written reflection. In contrast, Team B preferred to launch directly into discussion.

\section{RESEARCHER FLEXIBILITY AND RESPONSIVENESS IN THE FIELD}

Although I had a plan of how the fieldwork would proceed, the reality often differed from what was envisaged. Within the TBC approach, working collectively with multiple participants, both risks and consequences of unplanned elements disrupting plans were greater. Therefore I found it helpful to be as flexible as possible to meet participants' needs and address circumstances arising. Hui 1 is described as an example, highlighting original plans, situations that arose, and changes to plans.

The purpose of Hui 1 was to introduce FoK and share approaches for teachers to learn about students' FoK utilized in other studies. I envisaged running three Hui 1, one for each group of participants: students, parents and teachers, so I could pitch each 
session appropriately. However due to a range of reasons, including sickness, older students' intolerance with younger ones, and forgetting, twelve (48\%) participants formed alternative groupings for Hui 1 (see Appendix B). Notably, Team E attended Hui 1 as a team, and followed it immediately with Hui 2, in a two-hour session. However, although the number of iterations of Hui 1 and participant groupings differed from my plan, the format remained consistent.

\subsubsection{Ethical dilemmas}

Three ethical dilemmas described here relate specifically to the TBC methodology.

WHEN POTENTIAL PARTICIPANTS DID NOT FIT THE PLANNED TEAM STRUCTURE

In two teams, a dilemma emerged: what to do when willing participants did not form the desired configuration. In addressing these dilemmas, I considered the authentic relationship that was the foundation for teams, and my ethical responsibility towards willing participants. Therefore, I did not allow parent team members to participate without their son or daughter. Team E included three students, one of whom did not identify as Māori or Pasifika. In this case, Toby was invited in the hope that his involvement would make participation attractive to his Māori friend Shea, which indeed happened.

\section{REMINDERS OR COERCION? ABSENCE OR WITHDRAWAL?}

Early in fieldwork, some participants forgot to attend Hui 1, resulting in my scheduling additional Hui 1 events. Due to the potential consequences for me if this became a pattern during fieldwork, I began to provide individual notices on a termby-term basis. As well, I provided reminders closer to the time by email, text message, and voicemail. This strategy seemed largely successful, as illustrated in Appendix B, which summarizes attendance at research events. After Hui 1, very few meetings were rescheduled, and most research events were well attended, with two exceptions: FGs (especially SFGs) and attendance of parents who later withdrew.

As I provided reminders of team events, at times I was troubled by the potential for overstepping ethical boundaries. I wondered at what point providing reminders could be considered harassment, contravening the right to voluntary participation. I reflected on my rationale in my journal:

I'm trying to protect the integrity of the team structure - that's why l'm taking these actions to remind everyone. If they aren't there that's fine, that's their right, but l'm trying to make sure that if someone is absent it's not due to organizational lack on my part. (reflection, 22-06-11)

Some participants said they appreciated notices and reminders, but two parents (Carol and Lorena) who later withdrew stopped responding to these messages, 
giving me the dilemma of how to interpret the silence. In both cases, I made inquiries via text messages and eventually sent a letter asking for clarification, but neither responded. Later Lorena's son Piri confirmed her wish to withdraw.

Absence also created ethical dilemmas for me as the researcher, relating to my ideas about protecting the integrity of the team, and the principle of voluntary participation. When Thor and his mother Carol (Team A) were absent from Hui 3, I was unsure how to interpret this. Carol had stated in Hui 2 Thor was very keen to participate, and Thor himself had verified this. He also mentioned he waited at Carol's work each night to go home with her. Therefore, I wondered whether their absence could have been Carol's decision. Could Carol's wish to withdraw prevent Thor's attendance? Lizzie (Team A's teacher) also tended to this view. She mused "but he really did like to come didn't he, I genuinely got the feeling that he really enjoyed being a part of it" (TFG3, lines 340-342).

My attempts to contact Carol were unsuccessful, and eventually, interpreting this as withdrawal, I stopped trying. However, while attending a Team E event on 8 August (see Section 8.3.1), I unexpectedly saw Thor. I sensed his embarrassment from his body language, so I waited about 30 minutes and then approached him, hoping to ease his discomfort. He stated Carol wanted to withdraw because she was too busy, but he wanted to continue. When I telephoned Carol on 22 August, she confirmed withdrawal, and gave consent for Thor's continued participation. Lizzie subsequently organized TM1b so Thor could share his personal profile as planned.

\section{WITHDRAWAL OF A TEACHER}

Because each team was built around the authentic relationship between a teacher and students, it was particularly problematic when a teacher participant withdrew. This happened in Team D, when Paul decided to withdraw soon after Hui 3. I am grateful Paul permitted me to use data collected and participated in an individual interview, despite withdrawal. This effectively meant that he withdrew from the team, but not the research.

Participants' rights outlined in the information sheet allowed anyone to withdraw any time up to Hui 4, but Paul's withdrawal after Hui 3 posed ethical dilemmas. First and foremost, I was concerned for Piri's and Peter's wellbeing. At the beginning of the project, they had expressed their hope to gain a closer bond with Paul; I was concerned about what his withdrawal might mean to them. Secondly, I was concerned any possible feelings of rejection or anger could affect the classroom relationship and learning. Due to these concerns, I sought advice from my supervisor and a co-chairperson of the Faculty of Education Ethics Committee 
regarding how best to proceed. Consequently, contrary to norms that stipulate it is not appropriate to make continued requests of a participant who has withdrawn, I explained to Paul I wanted to minimize any possible negative impacts on their working relationship. We agreed to hold a TM, where Paul told the students he withdrew due to workload issues, and invited them to continue participation by carrying out the strategy agreed in Hui 3.

I wondered whether I could have avoided teacher withdrawal at such a relatively late stage of the project; however, the ethical principle of voluntary participation means the right of withdrawal is paramount.

\subsubsection{Supportive factors}

\subsubsection{Georgia, the mothership}

At the teacher recruitment meeting, Georgia offered to be my "mothership" at the school site, to support me with organization and communication. Some ways Georgia helped me included:

- acting as a conduit between student participants and me (e.g., giving notices to students);

- acting as a conduit between teacher participants and me (e.g., informing me about workload pressures, which led to shortening TFG2).

\subsubsection{Home base}

Planning the study, I knew that some participants may have negative feelings towards KHS, or schooling in general, and that the research venue would affect team dynamics. I invited venue suggestions, but the only venue suggested was the school. Georgia suggested a school meeting room used for ad hoc bookings, which was always available when I wanted it, and I was entrusted with a key to the building. Thus, for six months, I became like a member of the school community, and it felt like 'our room,' giving a strong message to participants about the high value that KHS placed on the research.

The meeting room's availability inspired me to routinely build in a fifteen minute buffer period before each one-hour research event. For instance, drinks and food were available from $4.45 \mathrm{pm}$ when a research event was scheduled for $5-6 \mathrm{pm}$. This helped me to provide a warm and welcoming environment, promote timely attendance and completion, and help team members develop bonds. 


\subsubsection{Resources for TFGs}

Two elements supported the effectiveness of TFGs. Firstly, in negotiating entry to the school, I stipulated that a condition of participation was school funding for teacher release time, to allow teachers' attendance at three half-day TFGs. Secondly, TFG1 and TFG2 were held in a nearby school, which provided convenience, privacy, and allowed teachers to give their undivided attention.

\subsection{Conclusion}

Common experiences across teams related to the implementation of strategies to learn about students' FoK highlight diverse ways to approach this aim, and benefits and challenges of this work. Therefore, findings suggest considerations for teachers undertaking to learn about their students' FoK, including strategy choice, management, facilitation, and timing. Learning about their Māori and Pasifika students was valuable for these NZ high school teachers and the students themselves, especially those who were previously more marginalized. Although some teachers adapted their pedagogical practice, findings suggest that this was the biggest challenge for teacher participants. 


\section{Chapter 10: Discussion}

In order really to change our education in Vygotsky's sense or actually put his theory into educational practice, a more collectivist interpretation of his ideas is needed. And that would mean, in particular... making the educational process more personal. (Ageyev, 2003, p. 446)

The move to a more participatory form of engagement characterised by an intended mutuality, a disposition to see difference as a potentially creative resource, and more overt commitment to co-construction require quite different relationships and spaces and a quite different conceptual and linguistic schema to frame such aspirations. (Fielding, 2007, p. 307)

A dialectical approach... emphasizes repeatedly that action and construing are inextricably connected... change involves a shift at both levels - action and construing... we need to be wary when there is only evidence of movement in one area and not the other. It is easy enough to talk about things in a different way, at least for a while. However, in order for change to be sustained, shifts in both areas are necessary. (Dallos, cited in Miller, 1996, p. 108)

This chapter concludes the thesis. To begin, the research purpose is restated, linking the study to social justice issues in New Zealand schooling, highlighting gaps in current literature, and identifying areas in which new knowledge is contributed. Secondly, outcomes from the study are presented, within two sections. In Section 10.2, guided by the research questions, findings are examined in relation to other scholarly work in the FoK field. Discussion highlights the complex nature of factors that positively or negatively affected valued outcomes achieved. In Section 10.3, discussion relates to elements of TBC which emerged as relevant to valued outcomes achieved, and the nature and significance of team systems. Following the two sections relating to research outcomes, limitations of the thesis are discussed. Finally, implications for research and teaching practice are presented.

The relevance of literature from other fields became apparent during analysis, including systems theory, student voice, and parent involvement, and these are integrated into discussion. Due to thesis constraints, integration of research from other fields is necessarily limited.

\subsection{Purpose and contribution of the study}

The study explored applicability of the FoK concept for Māori and Pasifika students in a New Zealand secondary school context. This focus was identified because, within the field of FoK, no study had previously related to a New Zealand high school context, and I found only twelve studies internationally which were conducted in high school settings. Also, the prevalence of deficit theorizing, less positive schooling experiences (Bishop et al., 2003), and poorer educational outcomes for Māori and Pasifika students (NZQA, 
2010), highlight their minoritized status within New Zealand's school system. Given the strong focus in government policies regarding national goals of inclusivity and social equity, the need for schools to become more effective for Māori and Pasifika students presents an urgent challenge (Hogg, 2008).

The study expands knowledge in the field of FoK. Results illuminate valued outcomes achieved and factors that support or constrain valued outcomes, when high school teachers learn about minoritized students' FoK. Involvement of student, parent, and teacher participants generated new knowledge highlighting participants' preferred strategies and perceptions of factors that supported or constrained valued outcomes. Although the goal was not identification of students' FoK, findings of diverse FoK emerged.

However, major contributions of this study relate to the application of team-based collaboration (TBC) to select strategies for identifying FoK preferred by Māori and Pasifika students, their parents, and teachers, and outcomes achieved. Results illuminate the potential of TBC to establish a setting in which new initiatives can be explored, within an alternative culture that transcends school norms. Benefits of successful TBC included development of trust and new understandings of each other for members, as well as implementation of strategies endorsed by participants (Hogg, 2013b). Finally, the study contributes a model which utilizes TBC as an approach to application of the FoK concept.

\subsection{Outcomes from teachers learning about Mãori and Pasifika students' FoK}

\subsubsection{Illumination of rich FoK}

Although this was not the focus of the study, results affirmed wide-ranging FoK held by Māori and Pasifika students and families, corroborating other findings of rich and diverse FoK in minoritized groups. Table 10.1 provides a selection of examples.

Table 10.1: Examples of students' FoK

\begin{tabular}{|c|c|c|c|c|}
\hline $\begin{array}{l}\text { Areas of } \\
\text { FoK }\end{array}$ & Sports & Performing Arts & Animals & Technology \\
\hline Examples & $\begin{array}{l}\text { Boxing; } \\
\text { Basketball; } \\
\text { Netball; } \\
\text { Rugby league; } \\
\text { Scootering. }\end{array}$ & $\begin{array}{l}\text { Cook Island } \\
\text { dancing and } \\
\text { drumming; } \\
\text { Kapa haka } \\
\text { performance; } \\
\text { Rock'n'roll } \\
\text { dancing. }\end{array}$ & $\begin{array}{l}\text { Pets (cats, dogs, } \\
\text { turtles, } \\
\text { possums); } \\
\text { Horses. }\end{array}$ & $\begin{array}{l}\text { Online gaming; } \\
\text { Cellphone use; } \\
\text { Powerpoint. }\end{array}$ \\
\hline
\end{tabular}


However, learning about students' FoK was not without challenges for teachers. As found by Rogers et al. (2004), the need for support to help low-SES students to recognize their FoK and its value was apparent. For example, in Team E, Shea was humble about his expertise, and David was unable to take the initiative in conversations about his FoK. Similarly, Team D students struggled to make movies about their own FoK, but finally managed to complete this task collaboratively and describe each other's FoK.

Other challenges to identifying students' FoK suggest benefits from selection of strategies suited to specific individuals and contexts. For instance, when students were quiet, shy, or lacking verbal confidence, it was difficult for teachers to learn about their FoK through verbal presentation or discussion. In a further example, making movies and teaching the class both involved high levels of skill and workload, as well as access to equipment.

Parents' participation provided a fuller perspective of students, and established mutual respect and trust in teacher-parent relationships. When parents participated, they supported students in interaction with the teacher and were a source of information about students' FoK. However, students participating without parents valued their absence, and Andrade (1994) warns against supplanting children's voices, because they "are capable of describing themselves and their lives in candid and lucid fashion" (p. 16).

Results affirm earlier findings from González et al. (2011), that trust has a key role when teachers learn about students' FoK; in this study, trust was also influential in the effectiveness of teacher engagement of team members. For highly marginalized students and participants with negative schooling experiences, trust in the teacher was a pre-requisite for participation. Access to more personal sites and sources of information were supported by increasing levels of trust between participants.

Teacher participants' experience in the study confirm González et al.'s (2011) argument that:

Teachers using the FoK approach need time, space and supportive networks for engaging with community knowledge, working through complexity, and putting their new understandings into action with communities and students. (p. 490)

Teacher participants gained support from three main areas as they explored application of the FoK concept to their work: from their team, each other, and me, the researcher. In TFGs, teachers collaboratively reflected on the meaning and implications of what they were learning about students' FoK, mirroring collective 
teacher reflection in study groups (such as in González et al., 2005). Learning about students' FoK by listening and observing them with new intent was challenging for teachers. Similar to the findings of González et al. (2011), mostly, teachers did not find the time to reflect on or review students' FoK apart from within these forums, suggesting the possible risk of lack of time to "theorize and explore such complexity productively" (González et al., 2011, p. 489). Thus, time constraints affected teachers' learning and valued outcomes achieved. This may account for differences between parents' and students' assessment of teachers' understanding of students' FoK, compared to teachers' self-assessment.

School funding provided teacher release from duties for TFGs for two half-day sessions, but this timeframe allowed collective engagement with only excerpts of material related to students' FoK. The modest resourcing of TFGs requested was manageable within the school budget, and took teachers away from their core business only twice, but the question remains: how would teachers' learning differ if more time was available? González et al.'s (2011) reminder, “understanding complexity - of communities, activities, identities, learning processes, knowledges and contexts - is important" ( $p$. 488) provides a challenge to findings - to what degree did appropriate complexity of knowledge result? González et al. (2011) argue that teacher participants' dismissals of students' FoK and invocation of deficit discourse are indicative of lack of successful engagement with complexity. According to these criteria, the work was unproductive for one out of five teacher participants. Other indicators also suggest that some understanding of complexity was achieved by four teacher participants. These include their statements that the dynamic nature of FoK meant that ongoing learning would be needed, and statements of intention to incorporate into practice greater communication with and learning from - families.

\subsubsection{Teacher-student relationships and effective learning behaviors}

Improved teacher-student relationships when teachers learnt about students' FoK were congruent with findings from other studies (e.g., Amanti, 2005; Riojas-Cortez \& Flores, 2009). The significance of teacher-student relationships is well established in international literature; widespread agreement verifies good teacher-student relationships enhance student motivation (e.g., Davis, 2003; Hullena \& Hullena, 2010; Wentzel, 1997); help create safe, supportive classroom environments (e.g., Lomax, 2007); and promote achievement (e.g., Cornelius-White, 2007; Nieto \& Bode, 2008).

Participants' perceptions of the fundamental importance of teacher-student relationship were consistent with findings related to Māori and Pasifika students and 
other minoritized groups, confirming that "relationships matter" (González et al., 2011 , p. 488). Māori students place a high value on reciprocal relationships with teachers (Bishop \& Berryman, 2006; Bishop et al., 2003; Nelson, Christensen, \& Cleary, 2008), as do Pasifika students in New Zealand (Hawk et al., 2002; Spiller, 2013). The importance students assign to close relationships with teachers, evidenced by stated hopes, affirms the value of teachers investing time in FoK inquiry, to go beyond policy imperatives of decontextualized skill and knowledge development (González et al., 2011), and add weight to calls for relational pedagogy (Sleeter, 2011).

Findings highlight the importance of personal elements within teacher-student interaction for individuals with collectivist values, which are important in the values framework of Māori, Samoan, and Cook Island cultures (Cullen, 2009). Therefore, this study confirms that teacher-student relationship is a potential site for cultural clashes, due to the dominance of White teachers. Depending on the extent that White teachers relate to their originating individualist cultures, they may "seem to be more comfortable with short-term interactions defined solely by social roles, norms, and other constraints of a given social interaction" (Ageyev, 2003, p. 444). Moreover, valued outcomes achieved when teachers learn about students' FoK highlight the need for teachers to break through constraints of task-focused interaction and traditional teacher-student relationship boundaries, to open up the possibility of dislodging deficit discourses through new knowledge gains.

Time and timing were important in relationship building. The study highlighted the value of learning about students' FoK early in their school careers. However, FoK inquiry also created valued outcomes for students near the end of schooling. To gain deep knowledge of students and maintain relevant, meaningful relationships, the dynamic nature of students' FoK requires ongoing investment of time. Inquiry into students' FoK was a labour-intensive process, but teams' strategy choices demonstrate the value participants placed on the work.

Close links between student-teacher relationships and effective learning behaviors evident highlight the importance of emotionality in learning, as postulated in sociocultural learning theory (Vygotsky, 1986). Results corroborate other findings of a positive relationship between students' and teachers' knowledge of each other and their commitment and efforts towards each other (Hensley, 2005; Mclntyre et al., 2001). Students' sense of belonging and participation rates improved when teachers drew on their FoK as a cultural resource for learning, thus attending to students' emotional state by demonstrating interest and care (Camnagian, 2010; Lee \& Quijada Cerecer, 2010; Yoon, 2012). Also, David's (Team E) new practice of 
drawing on FoK in creative writing suggested teacher interest affirmed its value and relevance at school (Comber \& Kamler, 2007).

As Vygotsky (1986) explained, affective, behavioral, and cognitive experiences are essentially inter-connected:

Thought is not begotten by thought; it is engendered by motivation, i.e., by our desires and needs, our interests and emotions. Behind every thought there is an affective-volitional tendency, which holds the answer to the last 'why' in the analysis of thinking. A true and full understanding of another's thought is possible only when we understand its affective-volitional basis. (p. 252)

Therefore, in considering links between learning about FoK, student-teacher relationships, and effective learning behaviors, I would argue when teachers learn about students' FoK - including their passions, activities, skills, and knowledge this effort creates relevant new knowledge about affective, behavioral and cognitive features of students' lifeworld experience.

FoK work resulted in self-reflection for teachers who participated in reciprocal sharing (Hensley, 2005). Team C suggested Georgia share aspects of her FoK with classes, to build connections with students, and as an entrée to activities drawing on their FoK. Learning about students' FoK and sharing his own caused Tom to reflect on himself as a cultural being, raising awareness of assumptions he made (Amanti, 2005) due to his cultural perspective, and reframing aspects of his family situation that were incongruent with his values. Teachers learnt about students' sociocultural context, including family as a source of students' FoK (Cremin et al., 2012; Floyd-Tenery, 2005), extending prior ideas about what is involved in getting to know students well.

Relationships between teachers and minoritized students and their families were supported by team size and structure. Selection of Māori and Pasifika students for participation within small team settings positioned these students so their voices could be heard, not only placing them centre stage, but also avoiding the dominance of middle class students' voices within whole class settings (Fielding, 2001). Inclusion of two or three students in each team, together with parental support, helped students avoid feeling overwhelmed (Levin, 2000). Also, small teams generated a focus on individuals, thus avoiding "presumed homogeneity" (Fielding, 2007, p. 306) regarding what students have to say, and establishing a manageable focus for teachers.

\subsubsection{Applying FoK to pedagogical practice}

Pedagogical applications of FoK relate to all three themes within Rodriguez's (2013) typology (see Table 2.3). One teacher identified the nature of curriculum content in her subject discipline (Classics) as a constraint to scaffolding learning with students' FoK, thus limiting application to Theme 1 . However, two teachers quickly recognized 
the value of integrating learning that drew on students' FoK and learnt how to design activities to achieve this. These substantial units of learning primarily related to students' FoK (Theme 2). Georgia's authentic activity in which students learning Japanese provided information about their FoK to help students in Japan learn about life in New Zealand was an example of a Theme 3 application.

Analysis of what was missing from data collected highlighted other possible pedagogical applications. For example, only one teacher developed a classroom application that sought to be transformative, by increasing the student's ability to effectively use agency in classroom situations, therefore going beyond mandated curriculum requirements. Most pedagogical applications implemented a sociocultural approach to learning. A possible reason was the nature of training; readings that teachers reflected on in TFGs were grounded in sociocultural learning theory. A second missing factor relates to pedagogical applications reported to provide higher levels of challenge to students. Shea stated teachers' knowledge of students' FoK should minimize assignment of "baby work" (interview, line 73). However, in Team $D$, as a result of the study, the students were provided easier work, indicating their teacher had not developed more positive beliefs or expectations for their learning. In Team E, the teacher's hopes related to strengthening personal connections and mutual commitment between teacher and students, and the exclusion of learning activities that drew on students' FoK in valued outcomes reflected this vision.

Mandated curriculum and assessment requirements demanded teachers' attention, threatening teachers' efforts towards pedagogical innovation and influencing ideas about how to relate FoK to class settings. However, as teachers experimented in applying students' FoK to classroom pedagogical practice, they became adept at doing this in ways that were congruent within teaching and learning programs.

In conclusion, results verify potential benefits of application of the FoK concept as a worthwhile approach to multicultural education (Erickson, 2007; Moll \& González, 2004; Nieto \& Bode, 2008; Sleeter, 2005). Pedagogical application of FoK provides a potential model for enacting a sociocultural approach to teaching and learning that addresses students' affective and cognitive needs. Therefore, teacher learning about FoK is a potential approach to achieving respectful, collaborative home-school partnerships with families of minoritized students that can improve the effectiveness of schooling experiences (Allen, 2007; Amatea, 2009; Winn-Tutwiler, 2005). Affectively, it is a way to provide caring, relational pedagogy (Noddings, 1984; Sleeter, 2011; Vygotsky, 1986). Cognitively, it enables students to develop academic skills through teacher encouragement and helps to draw on FoK for completion of meaningful tasks within a program of academic learning (e.g., Bailey, 
2011; Calabrese Barton \& Tan, 2009; Camangian, 2010; Carbone, 2012; Lee, 2001; Pirbhai-Illich, 2010).

A distinctive feature of the FoK approach compared to other approaches to caring or relational pedagogy is a focus on a processual notion of culture, to capture the dynamic complexity of people's lives in today's connected and increasingly shrinking world (González, 2005). Application of a processual view of culture allows identification of wide-ranging areas and sources of knowledge that generate hybridized FoK. Therefore, a point of difference is the theoretical framework which underpins FoK scholarship, as distinct from New Zealand studies which report findings about ways that Māori and Pasifika students learn and approaches which best suit their needs (such as Bishop et al., 2003; Spiller, 2013).

\subsection{Outcomes from the team-based collaboration approach (TBC)}

\subsubsection{Elements of TBC}

The establishment of norms and practices within teams resulted in unique and distinct team milieu, the character of which related to decisions about strategies for implementation and valued outcomes achieved.

\subsubsection{A conceptual map to summarize elements of TBC}

Elements that influenced the nature of each team's milieu included factors related to purpose, participants, and process. Although all teams were charged with the same purpose, each team developed its own culture, with its own norms and practices, as described in Table 9.1. Team norms and practices were a complex interplay between team participants, process, and purpose, as shown in the conceptual map (Figure 10.1) (Hogg, 2013b).

Participant factors related to individuals within teams, including me. These included: identity (student, parent, teacher, researcher); initial relationships between members (nature and quality); FoK (including experiences participating with equal status in open discussion); values (personal principles and priorities); number of members; and beliefs, attitudes and dispositions held.

Process factors related to actions, including all elements of the cyclical process undertaken by participants. Process factors included actions taken by team members and/or experienced by them. Process factors identified in the conceptual map affecting team culture were: researcher role (participant observer role); training (provided by researcher to participants, such as Hui 1, TFGs, and other material and support as requested, and other TPL); facilitation techniques (including aim, 
approach, and who contributed to facilitation); inclusion strategies (words and actions which affirmed members' presence and contributions, and built positive relationships between members); decision-making process (how strategies were agreed); teacher positioning (stance or role the teacher took within the team);

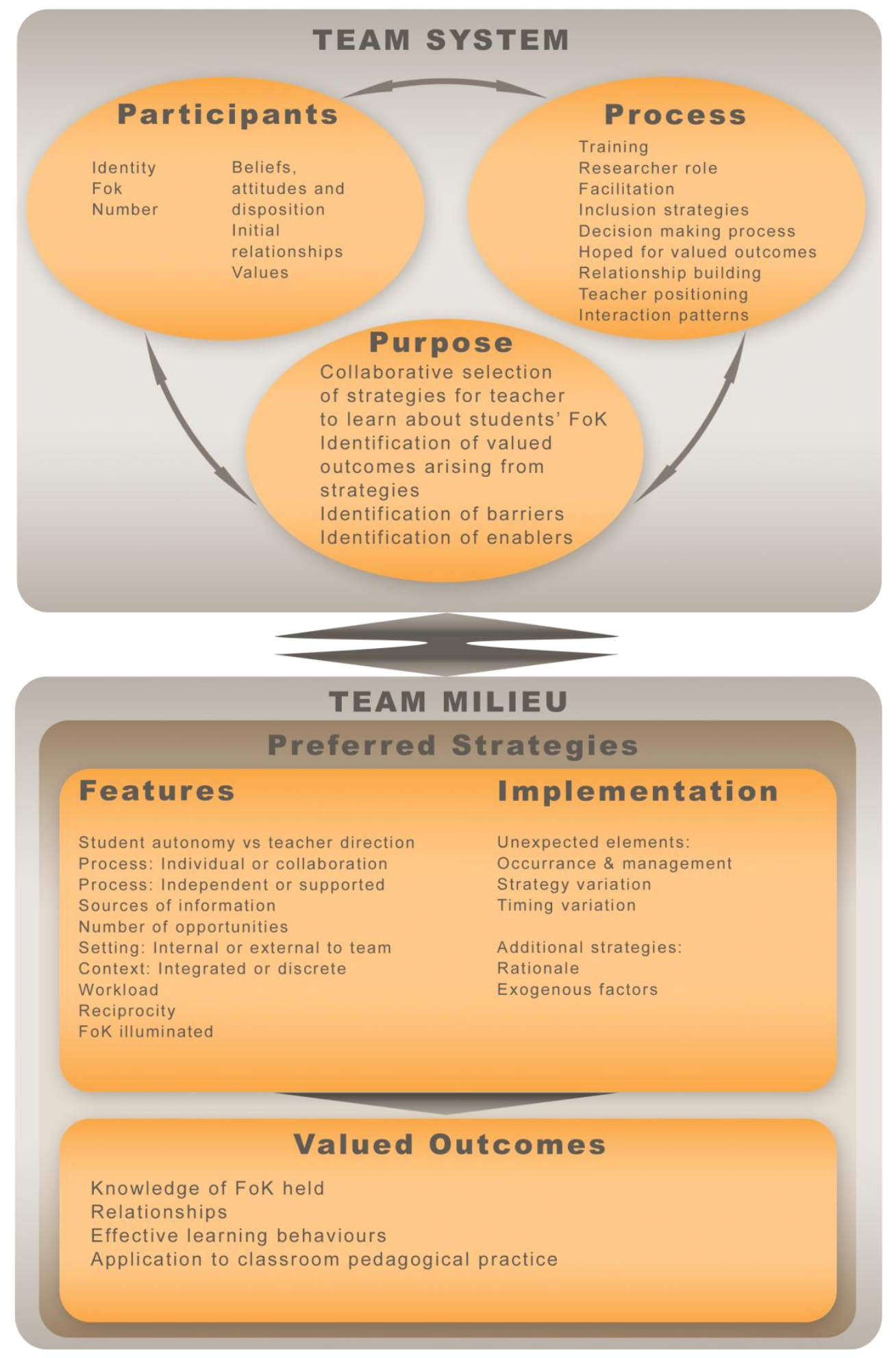

Figure 10.1: Conceptual map: Elements of the TBC approach 
interaction patterns (content and how each member was involved); and hoped-for valued outcomes (recorded by participants after initial training and during the study).

Four factors were identified relating to purpose, associated with the research questions. The first factor was collaborative selection of strategies for the teacher to learn about students' FoK, and other factors related to identifying valued outcomes arising from strategies implemented, and elements that enabled or constrained valued outcomes achieved.

Cross-case analysis suggested that interplay between factors related to participants, process, and purpose generated distinctive team milieu for each team. The character of team milieu impacted on decisions and implementation of strategies as well as valued outcomes achieved.

\subsubsection{Application of conceptual map to Team D}

In this section, the relevance of the conceptual map is demonstrated by its application to discuss Team D findings.

In Team D, various factors combined to create an unhelpful team milieu. Participant factors included Paul's belief that he needed to learn about challenges the students faced at home, so he could develop empathy, negatively affecting his openness to learn about their FoK. Participant factors associated with students that contributed to the ineffective team milieu were their self-management skills and lack of prior experience in open discussion with adults.

Process factors that contributed negatively included the decision-making process, because Hui 2's agreed strategy did not have the students' endorsement - their agreement was more in the nature of a concession. Teacher positioning also played a part: Paul's stance related to his stated lack of agency as a teacher. He suggested he could not allow the students to teach the class something because it would not be a coherent part of the IS teaching and learning program. Also he believed authentic sharing of students' FoK for the purposes of the research should be unsupported. A further process factor was the interaction pattern of things unsaid. Examples included Paul's unstated expectations relating to strategy implementation and avoidance of communication of negative feelings, such as his frustration and uncertainty when the students failed to honour the team agreement. Also, my facilitation included lengthy description of other teams' chosen strategies, limiting time available to talk about the students' preferred option. A further unhelpful factor may have been my practice of directing questions to students, inadvertently placing the burden of argument and agreement unduly on their shoulders. 
Within this team milieu, agreement and implementation of strategies were problematic, and valued outcomes achieved were minimal. The agreed strategy did not have all members' backing. Implementation problems included ongoing delays with the first strategy, and no illumination of FoK from the second. Ultimately, Paul learnt the boys liked to joke and became more tolerant of this. He also started to provide them easier work, highlighting that participation did not result in enhanced ideas about their strengths and skills.

\subsubsection{Teams were systems that transcended school and family cultures}

\subsubsection{Presentation of a model of team systems}

Each team can be understood as a system that overlapped family and school systems (Hogg, 2013b). Findings highlighted similarities with other collaborative groups in which stakeholders formed a partnership with the purpose of collaborative implementation of a strategy (De Shazer, 1982; Miller, 1996). This section therefore describes and discusses key elements and qualities of teams as systems.

Teams formed around me as the researcher, an outsider who brought knowledge of FoK scholarship, university research culture, and the invitation to form teams to explore application of FoK. Figure 10.2 is an adaptation of Miller's (1994) model, showing the relationship between home and school systems prior to the study, and the way team systems overlapped family and school system boundaries.

Similar temporary systems formed around family therapists and client family members for their work's duration (De Shazer, 1982), as well as educational psychologists (EPs) working with teachers, family members, and students with extreme behavior issues (Miller, 1996). The "temporary and overlapping system" (Miller, 1996, p. 106) allowed members to transcend boundaries defined by school culture, because they could "adhere to both sets of norms and values simultaneously, even when these are contradictory" (p. 202). In temporary systems which transcend school and home systems, Miller observed that:

as a result of these new norms it becomes possible to reconstrue children and parents... not only, then, does the new system create a partnership within which it is possible for members to reconstrue each other, thus adding implement a joint strategy, it also creates a level of personal relationship to the strategy itself in the manner Dallos sees as essential for change within a system. (p. 108)

Thus, the team structure and TBC along with teacher learning about students' FoK provided another layer of opportunity for enhanced personal relationships between members. Therefore, benefits enjoyed by students - improvements in teacherstudent relationships and effective learning behaviors - were influenced not only by 
learning about students' FoK, but also by students' increased feelings of relatedness and belonging when teachers valued their ideas (Ostermann, 2000), and gains in self-worth from the opportunity to contribute meaningfully for the good of others (Rudduck, 2007). Similarly, not only learning about students' FoK, but also participating in TBC supported teachers to: develop new perceptions of students' capabilities; see the familiar with 'fresh eyes'; be open to changes in thinking and practice; make concrete plans for improvement; and develop confidence in partnership-style relationships with students (Rudduck, 2007).

Discussion of team elements also must include consideration of the researcher who established the team. Two aspects are discussed here: administrative work and behaviors demonstrating my theories in action (Argyris \& Schön, 1974).

Teacher participants valued administrative support to organize complex logistical aspects of teamwork, so they could simply attend and give attention to the members and task at hand without time-consuming preparation. Logistical tasks included gathering information, scheduling, catering, communication, facilitation, transportation, and organization of materials. Multiple responsibilities and commitments of Māori and Pasifika students, their parents, and their teachers called for flexibility and understanding, as well as shared commitment to achieve effective working teams. Therefore, although four teachers expressed their intention to incorporate aspects of TBC into their future work, I wonder what resourcing might be required to make this approach manageable and sustainable.

Teacher participants reported my attitude and behavior in fieldwork influenced their own actions. My demonstration of care for participants established an ethic of care in teams that teachers stated inspired their commitment. My values and beliefs were also applied in commonalities regarding teams' goals, processes, and participants (illustrated in Figure 10.1), generating overarching norms and values that were divergent from school culture. For example, an overarching value was democratic processes, taught but not embraced in school practice (Rudduck \& Fielding, 2006). TBC provided "a legitimate and valued space within which students can speak" (Cook-Sather, 2002, p. 4), and extended the invitation to their parents.

Systems theory analysis postulates that school organizational cultures create barriers to innovation, regardless of espoused ideals and values enshrined in school goals and policies. Schools have an "orientation towards homeostatis, a tendency to maintain internal stability" (Miller, 1996, p. 99). Schools' preference for the status quo arises from their ongoing need to define, justify, and protect their domain of activity and power (Rice, 1969). For example, although teachers valued the 
democratic framework TBC provided, an aspect of school norms and values that proved hard to shift was teacher-student interaction with teacher as active/ in control and student as passive/ controlled. Therefore, providing "recognition of different voices as well as fair distribution of resources that provide the condition for equal participation (Ranson, 2000, p. 265) was not straightforward.

(a)

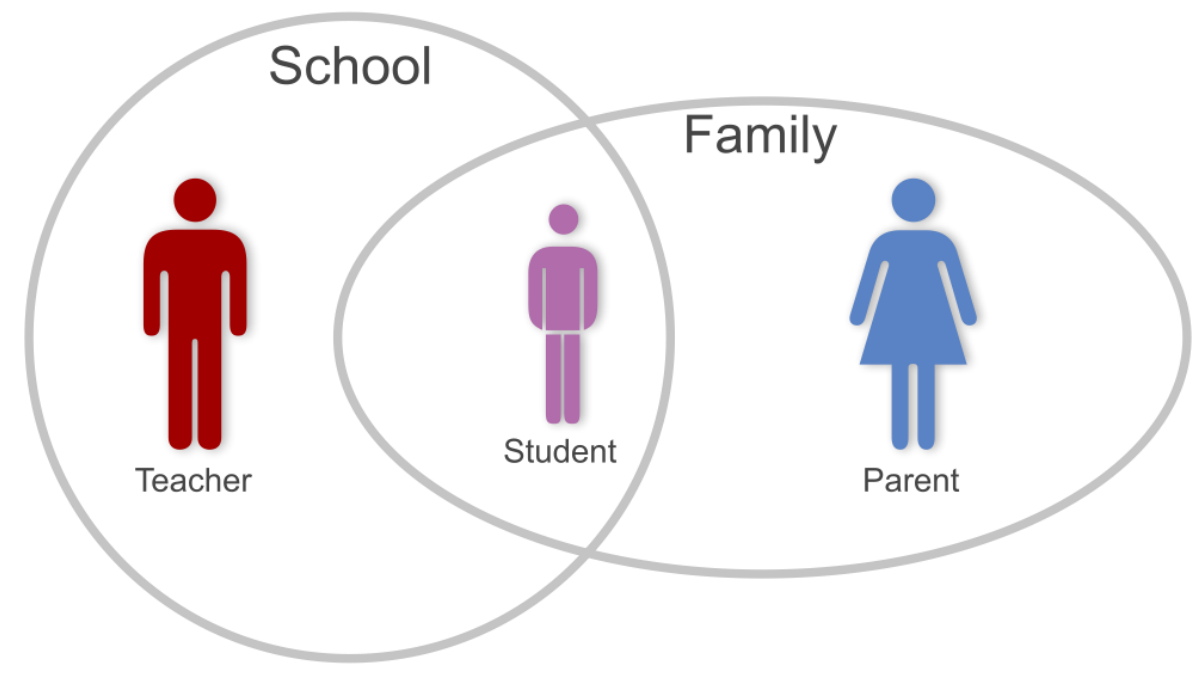

(b)

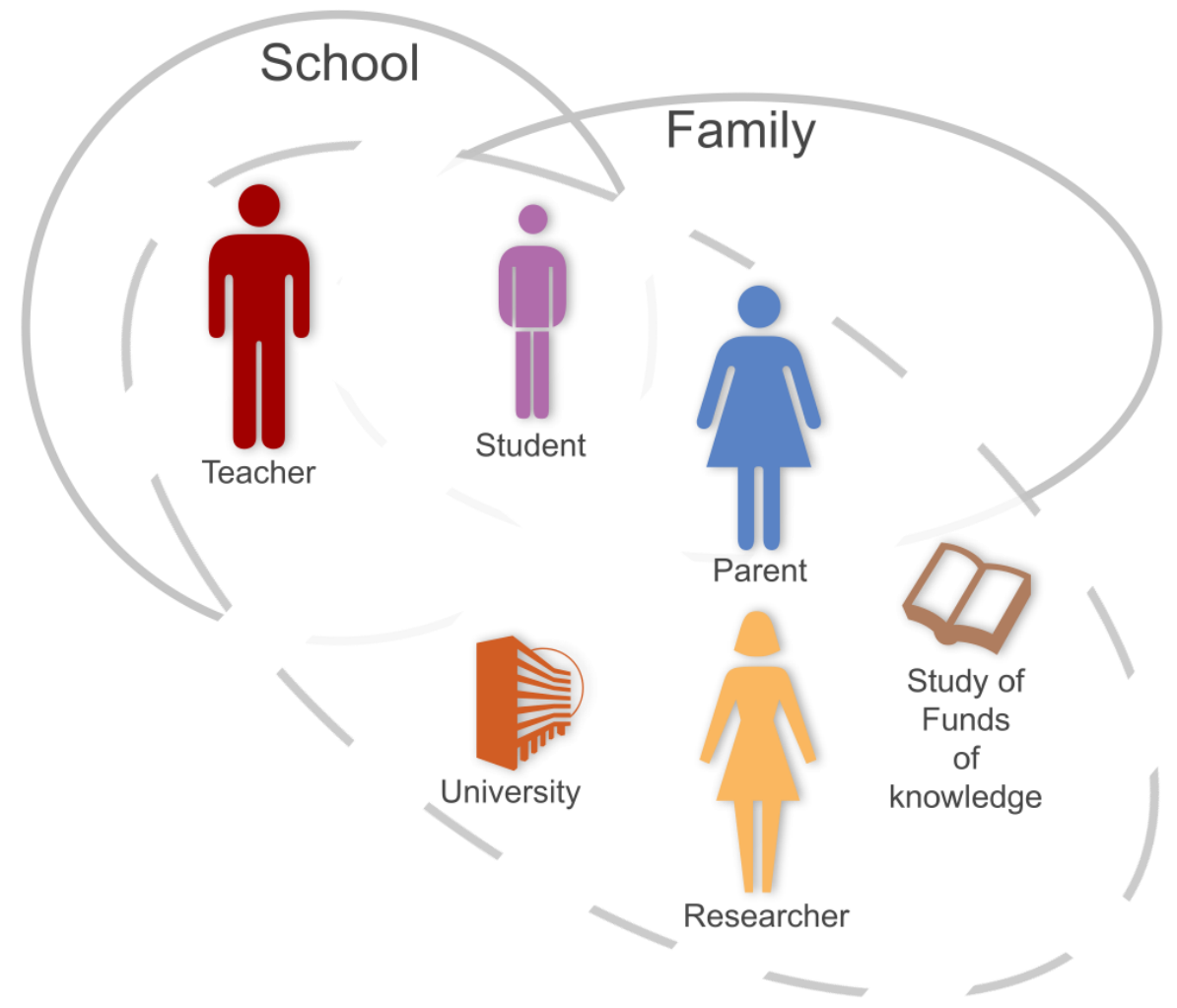

Figure 10.2 The location and nature of the temporary overlapping boundary

(a) The student as a member of the family and school systems;

(b) The introduction of the temporary overlapping system (after Miller, 1994). 
TBC created a small-scale setting beyond school culture in which it was safe to explore a new concept - FoK - and its application in KHS. Within each team, participants progressed their learning according to their zone of proximal development (Vygotsky, 1978), experimented, and built confidence. With collaborative support of team members, teachers could explore ways to apply FoK in their current classroom contexts. Therefore, approaching exploration from teams provided a safety zone that related to teachers' professional context but also bypassed school culture.

Miller (1996) found that, following successful intervention and subsequent dissolution of systems around EPs, school culture constrained widespread implementation. After removal of temporary systems, changes in practice were limited to teachers who participated in temporary systems with EPs, and school cultural norms remained intact regardless of changes achieved within the temporary system. Thus, when interventions were initiated within boundaries of an alternative system, they were not regarded as inconsistent or threatening (Miller, 1996). Thus, application of systems theory analysis suggests that TBC offers the possibility of achieving innovation and transformation by involving teachers in temporary systems offering new norms and practices, established around external agents. However, in this study no data related to events occurring after teams' dissolution. Therefore, I am unable to fully test application of Miller's (1996) theory.

TBC provided a space that allowed participants with an authentic interest in the students' schooling experience to contribute and take ownership of the forum. In centering team discussion around their agreement about how the teacher would learn about student participants' FoK, the discussion frame was tightly contextualized: the focus related to strategies to be implemented with them personally, or for individuals in their family, which may have enhanced students' and parents' confidence to contribute. Authentic relationships between team members allowed a meaningful focus on students and enabled parents to contribute as partners, thus providing an attractive environment for parent involvement (Mapp, 2003), enhanced by the solution-focused task (Hornby, 2000).

The team purpose and task was a supportive factor in establishment of respectful relationships between members. Key features were TBC's reciprocal and exploratory nature, which addressed Fielding's (2007) recommendations regarding engagement of student voice. Fielding's (2007) vision of future student voice scholarship seems to relate to various team features, including focus on dialogue, mutuality, collaboration, and attention to relationships, highlighting the potential of TBC. Fielding warns against "a consensus-based approach that tends to slip too 
easily into a premature resolution of difference" (p. 307), highlighting the importance of decision-making processes which pay due attention to the complexity of multiple perspectives, are democratic, and avoid reflecting traditional teacher-student-parent power relationships. The experience of this challenge highlighted its complexity for participants socialized into specific interaction patterns according to school culture. Despite identification of democratic processes as central, traditional teacher-student power dynamics created a threat to this principle, illuminating potential benefits from additional participant training (Mitra, 2009).

Although initial training was helpful, it was insufficient to address all barriers to valued outcomes. Initial training provided participants with knowledge about the nature of FoK (as defined within the study), and some strategies to learn about FoK. Training provided a foundation from which team members could participate in discussion and negotiation of strategies.

However, provision of relevant background knowledge of FoK was insufficient to ensure equitable participation. Some students were disempowered by lack of language (Mitra, 2009), or lack of related prior experiences:

For many young people who have not participated extensively at home or at school in open discussions or small group conversations,... and as planners and thinking partners, their facility with certain language structures lies dormant. (Heath, 2004, p. 53)

Therefore, qualities such as reticence and lack of verbal confidence constrained participation in TBC, as well as verbal sharing of information about FoK. Future applications of TBC would benefit from training for students to learn how to exercise their agency (Levin, 2000). Also, teachers and researchers would benefit from training to avoid middle class practices that are not inclusive (Bastiani, 1993).

\subsubsection{Skirmishes at the boundary}

Viewing each team as a system offers a possible interpretation of various incidents, which can be understood as system boundary issues, illustrating boundary tensions between school and team systems. Firstly, by vetoing her initiative regarding Troy's daily report, Kate's colleagues can be seen to be preserving homeostatis. Secondly, complaints from other students about Tom's presence at the $\mathrm{Cl}$ group practice can be understood as expression of feelings of individuals who were external to the system and did not share its norms and values. Thirdly, Tom's comment about his willingness to undertake the labour-intensive strategies agreed by Team E, despite his colleague's horror at the time involved, can be understood as acceptable within the boundaries of the temporary team system. Fourthly, this analysis offers an explanation of Team E students' reversion to previous student-teacher interaction 
patterns within the classroom. Also, Paul's frustration and withdrawal can be reframed in this analysis as resulting from differences between his team experience and sense-making of students' FoK compared with others. Conversely, for the other four teachers, shared values possibly strengthened their identity as a reference group of like-minded people, by functioning as a "frame of reference" (Nias, 1985, p. $107)$, as well as the effectiveness of their collaborative reflection.

Furthermore, Miller's (1996) theory offers a new way of looking at Anna's reluctance to share findings with the wider staff. A systems theory lens suggests that this could relate to maintenance of the school status quo, despite Anna's statement that the school needed to know the answers to my research questions.

\subsection{Influence of other contextual factors on findings}

All five cases were located in KHS, NZ, raising questions regarding the influence of school culture and NZ educational culture on findings. Figure 10.4 illustrates philosophical alignment between three key elements of the study - its goal, process, and participants - with elements of school policies and procedures, and national educational policies. Therefore, what was the significance of congruence between espoused values held within KHS, NZ education in general, and the present study? Although data do not address this question, arguably coherence of teachers' own espoused theories with those of the study may have influenced consent to participate. Teacher participants' previous voluntary involvement in TPL to improve Māori students' achievement suggests commitment to this aim. Also, the present study may have benefitted from alignment with national directives, allowing it to be perceived as a possible approach to meet professional requirements. Thus, as noted earlier, TBC provided a safe zone for TPL related to national and local educational goals, which bypassed influences of theories in action (Argyris \& Schön, 1974) evident in school culture.

Two points of similarity and tension were apparent between the present study and school TPL. A distinctive aspect of the FoK concept incongruent with other schoolbased TPL related to notions of culture. Although the E Tipu E Rea TPI program also aimed to improve effective teaching and learning for Māori students, the present study involved an emphasis on knowledge of individual students, rather than qualities, ideas, and needs of Māori students as a group. Therefore, E Tipu E Rea TPL and this study addressed aspirations expressed in Ka Hikitia in different ways. Secondly, the school's practice of drawing on data from target students to inform ongoing teaching decisions also involved learning from students. However, data collected from target students focused on perceptions of pedagogical practice, 


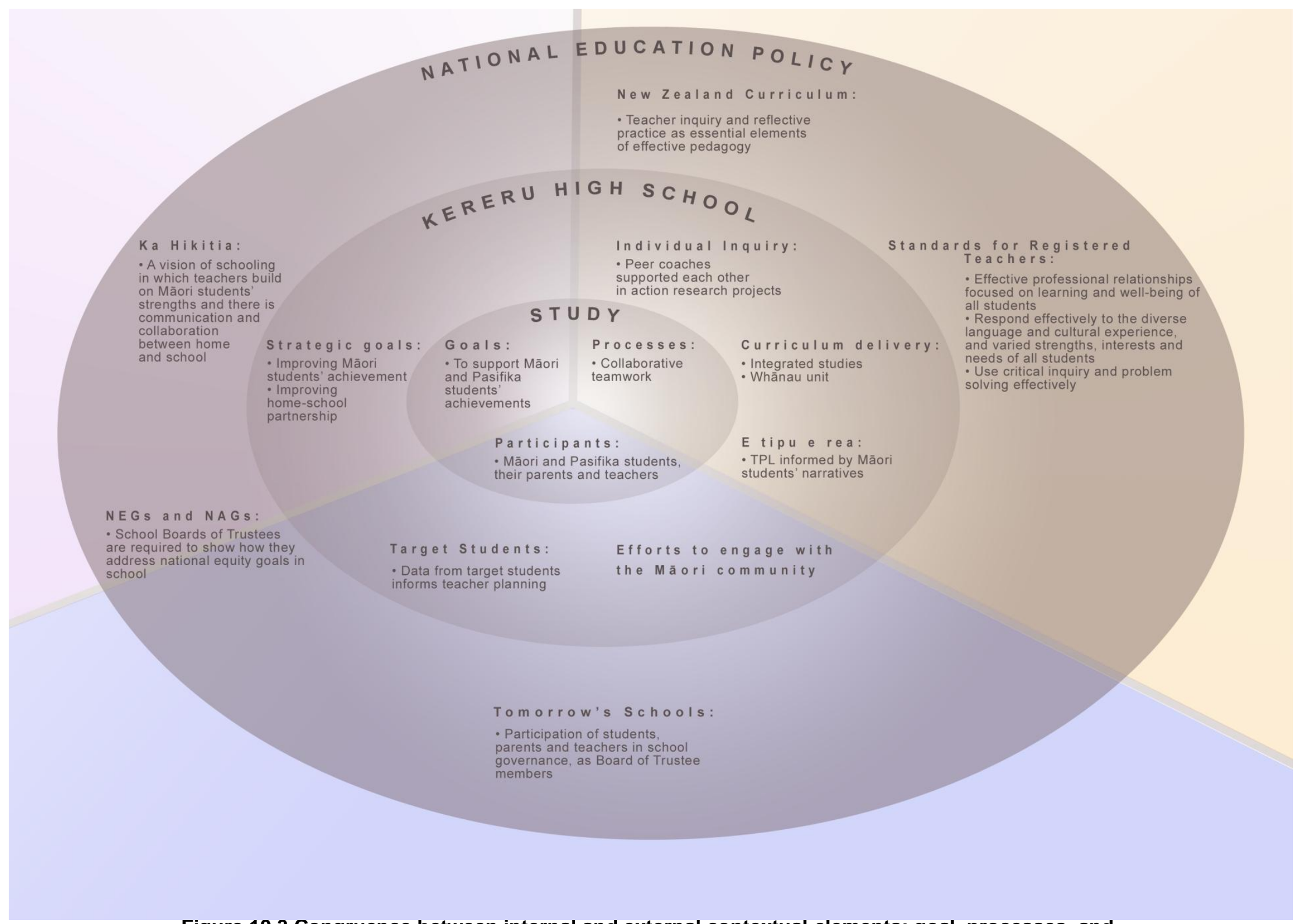

Figure 10.3 Congruence between internal and external contextual elements: goal, processes, and participants 
rather than inquiring into students' FoK. One consequence of the FoK reliance on theorizing from individuals was a concern of teacher and parent participants about the manageability and sustainability of learning about individual students' FoK.

\subsection{Limitations of the study}

As a human research tool, I brought subjectivity to the study, affecting the study design and data collected in fieldwork. A range of processes such as explicit identification of my position, fieldwork procedures, reflexivity, and careful analysis of my role were intended to preserve the study's trustworthiness (see Section 3.10). However, how I approached and enacted the researcher role is relevant to the issue of whether findings would transfer across contexts.

As noted, all five cases were located in KHS, raising questions about the degree to which setting implicated results. The research setting generated a large number of contextual variables (described in Section 1.4), the significance of which remains unclear due to absence of comparative data from another school setting. For example, I wonder how difficult it would have been for students to co-construct a Science unit of learning that was defined differently.

Analysis of participant characteristics suggests limitations arising from the nature of the sample. Four examples are provided here. Firstly, although five cases were examined, the sample was small. Secondly, only one student participant was female. Thirdly, although parent participants were the parents of Māori and Pasifika students, only Team B parents shared their son's/daughter's Māori or Pasifika heritage. Fourthly, teacher participants taught English, Science, Classics, and Japanese. However, there was a lack of teacher participants from other subject disciplines. Also, although the study offers rich description of some classroom applications of FoK in different subject areas, these related to topics being taught within the fieldwork period. Finally, teaching and learning programs for four teams were within an IS framework, possibly influencing findings.

All participants volunteered for the study. As teacher volunteers generally tend to be early implementers of new initiatives (Guskey, 1988), it is improbable that alternative sampling would replicate the findings. Teacher participants used different methods to choose students, with implications for parent participants. Team experiences were affected by team milieu. Therefore an element that makes replication challenging is the exact nature and dynamics of participants within teams. 
Demographic baseline data excluded some possible items (see Table 3.2). Consequently, findings do not highlight potential complexities associated with aspects of identity such as (dis)ability, sexuality, citizenship, or religious affiliation.

Baseline data included demographic information about the student population, aggregated student achievement data and strategic goals, as well as TPL policies, procedures and themes. However, data collection excluded school policy documentation related to other themes, such as student discipline. Moreover, my data collection focus on espoused theory (Argyris \& Schön, 1974) of the school (i.e., policy) did not capture the school culture. Although some data related to school culture, the lack of a strategically planned focus on this contextual information meant that any analysis would be incomplete, and data were insufficient to understand possible links between school culture and results.

Data collection methods did not systematically include primary sources related to classroom practice, such as classroom observations, lesson plans, or teaching materials. Samples of student work were limited. Although team hui and interviews provided rich triangulated data about pedagogical practices related to team agreements and teachers' statements about future intentions, the scope of the study excluded teachers' practices after teamwork concluded.

In fieldwork, although I made some use of team hui, focus groups and interviews to question participants about earlier comments and actions, questions and uncertainties remain concerning meaning of some data. Reasons include: lack of time to ask all possible questions in data collection; and tension between relatively open questions (such as 'Tell me how you felt about what your team did?' And 'How was that for you?') and closed questions (such as 'Why did you decide to interview the students during TMs?' and 'How did you feel when your teacher started to interview you during the TMs?'). I tended to favor open questions which did not lead participants, but the risk was that participants may not express all their thoughts and ideas, as they may not think of them at the time.

As explained in Section 3.8.5, I relied on thematic coding analysis, thematic analysis, and structural analysis to interpret data. However, other approaches such as discourse analysis, or analysis according to Ting-Toomey and Oetzel's (2001) intercultural communication model may yield new insights into team dynamics. To an extent, these decisions were affected by my status as an emerging researcher, including my awareness of available resources. For instance, when I engaged transcribers' services, none asked what technique to apply. Some inconsistency of transcriptions occurred, because some transcribers summarized data they 
considered irrelevant, and others transcribed all spoken text. With the limited time available, I could not complete all transcriptions fully, but I drew on audio- and videorecordings to consider all data.

\subsection{Implications of findings}

\subsubsection{Implications for research}

In conclusion, recommendations for future research include:

- Further study of application of the TBC approach;

- Further investigation of valued outcomes arising from teacher inquiry into students' FoK in diverse high school contexts;

- Further research relating to implications of strategy decision-making and choice on teacher learning about students' FoK;

- Further investigation of support which may help teachers to effectively draw on the FoK concept to enhance their professional practice;

- Investigation of long-term impacts on teacher beliefs and practices arising from learning about students' FoK.

\subsubsection{Implications for teaching practice}

In conclusion, recommendations for secondary teaching practice include:

- Confirmation of the value of inquiry into individual students' FoK, drawing on a processual understanding of culture;

- Undertaking inquiry into students' FoK within small team settings;

- $\quad$ TBC to agree preferred strategies to learn about students' FoK, with involvement of an external researcher;

- Participant training to enhance inclusiveness of TBC;

- Personalization of approaches to learning about students' FoK to suit participants and the teaching context;

- Provision of support, time, and collaborative reflection for teachers involved in TBC and learning about students' FoK;

- Integration of pedagogical strategies which support students to identify and effectively communicate their FoK, as authentic and congruent elements of teaching and learning programs;

- Integration of pedagogical strategies which support students to draw on their FoK to develop new academic knowledge and skills. 


\subsection{Conclusion}

Application of the FoK concept can help teachers break through limited understanding and inaccurate ideas of minoritized students which are prevalent with traditional teacher-student interaction, impacting positively on teacher-student relationships, effective learning behaviors, and pedagogical practice. Barriers that constrain learning about students' FoK include mismatch of selected strategy, participants, and context; need for training and resourcing for teachers undertaking FoK work; and constraints arising from mandated requirements.

Positive valued outcomes from both TBC and teacher learning about students' FoK affirm their worth for application with minoritized students, to progress social justice aims. Results from TBC depend on interplay between purpose, participant, and process factors. Collectively and separately, TBC and teacher learning about students' FoK support enacting both caring and pedagogical elements of sociocultural learning theory. Finally, as Tappan (1998) stressed, in his synthesis of Nodding's (1984) and Vygotsky's (1986) theories, both highlighted:

the enormous value, with respect to cognitive, intellectual, social, emotional, and moral development, that comes from experiencing close relationships and meaningful dialogue with others. (p. 31) 


\section{References}

Adds, P., Hall, M., Higgins, R., \& Higgins, T. R. (2011). Ask the posts of our house: Using cultural spaces to encourage quality learning in higher education. Teaching in Higher Education, 16(5), 541-551.

Ageyev, V. S. (2003). Vygotsky in the mirror of cultural interpretations. In A. Kozulin, B. Gindis, V. S. Ageyev, \& S. M. Miller (Eds.), Vygotsky's educational theory in cultural context (pp. 432-449). Cambridge: Cambridge University Press.

Aguilar, T. E., \& Pohan, C. A. (1996). Using a constructivist approach to challenge pre-service teachers' thinking about diversity education. In F. A. Rios (Ed.), Teacher thinking in cultural contexts (pp. 260-281). New York: State University of New York Press.

Allen, J. B. (2007). Creating welcoming schools: A practical guide to home-school partnerships with diverse families. New York: Teachers College Press.

Altheide, D. L., \& Johnson, J. M. (1994). Criteria for assessing interpretive validity in qualitative research. In N. K. Denzin \& Y. S. Lincoln (Eds.), Handbook of qualitative research (pp. 485-499). Newbury Park, CA: Sage.

Alton-Lee, A. (2003). Quality teaching for diverse students in schooling: Best evidence synthesis. Wellington: Ministry of Education.

Amanti, C. (2005). Beyond a beads and feathers approach. In N. González, L. C. Moll, \& C. Amanti (Eds.), Funds of knowledge: Theorizing practices in households, communities, and classrooms (pp. 131-142). Mahwah, NJ: Lawrence Erlbaum.

Amatea, E. S. (2009). Building culturally responsive family-school relationships. Upper Saddle River, NJ: Pearson.

Andrade, R. (1994). Children's constructive social worlds: Existential lives in the balance, unpublished doctoral dissertation, University of Arizona, Tucson, USA.

Andrews J., \& Yee, W. C. (2006). Children's funds of knowledge and their real life activities: Two minority ethnic children learning in out-of-school contexts in the UK. Educational Review, 58(4), 435-449.

Antrop-González, R., \& De Jesus, A. (2006). Toward a theory of critical care in urban small school reform: Examining structures and pedagogies of caring in two Latino community-based schools. International Journal of Qualitative Studies in Education, 19(4), 409-433.

Argyris, C., \& Schön, D. A. (1974). Theory in practice: Increasing professional effectiveness. San Francisco: Jossey-Bass.

Arksey, H., \& Knight, P. (1999). Interviewing for social scientists. London: Sage.

Ayers, M., Fonseca, J. D., Andrade, R., \& Civil, M. (2001). Creating learning communities: The "Build your dream house" unit. In E. Mclntyre, A. Rosebery, \& N. González (Eds.), Classroom diversity: Connecting curriculum to students' lives (pp. 92-99). Portsmouth, NH: Heinemann.

Bailey, B. (2011). 'When I make a film, it's out of my head': Expressing emotions and healing through digital filmmaking in the classroom. Digital Culture and Education, 3(2), 76-97.

Baker, D., Street, B., \& Tomlin, A. (2001). In J. Winter (Ed.), Understanding home school relations in numeracy. Proceedings of the British Society for Research 
into Learning Mathematics, 21(2), July 2001. Retrieved February 24, 2013, from http://www.bsrlm.org.uk/IPs/ip21-2/BSRLM-IP-21-2-9.pdf.

Ballenger, C. (1999). Teaching other people's children: Literacy and learning in a bilingual classroom. Columbia University: Teachers College Press.

Banks, J. A. (2001). Cultural diversity and education: Foundations, curriculum, and teaching $\left(4^{\text {th }}\right.$ ed.). Needham Heights, MA: Allyn \& Bacon.

Banks, J. A. (2007). Multicultural education: Characteristics and goals. In J. A. Banks \& C. A. M. Banks (Eds.), Multicultural education: Issues and perspectives $\left(6^{\text {th }}\right.$ ed., pp. 3-30). Hoboken, NJ: John Wiley.

Baquedano-López, P., Alexander, R. A., \& Hernández, S. J. (2013). Equity issues in parental and community involvement in schools: What teacher educators need to know. Review of Educational Research, 37, 149-182.

Baskerville, D., \& Bondy, A. (2010). A bicultural approach to teaching and learning for diversity. In V. Green \& S. Cherrington (Eds.), Delving into diversity: An international exploration of issues of diversity in education (pp. 35-44). New York: Nova Science.

Bastiani, J. (1993). Parents as partners: Genuine progress or empty rhetoric? In P. Munn (Ed.), Parents and schools: Customers, managers, or partners? (pp. 101-116). London: Routledge.

Basu, S. J., \& Calabrese Barton, A. (2007). Developing a sustained interest in science among urban minority youth. Journal of Research in Science Teaching, 44(3), 466-489.

Belgrave, F. Z., \& Allison, K. W. (2006). African American psychology: From Africa to America. Thousand Oaks, CA: Sage.

Bhabha, H. K. (1994). The location of culture. New York: Routledge.

Bishop, R. (2005). Pathologizing the lived experiences of the indigenous Māori people of Aotearoa New Zealand. In C. M. Shields, R. Bishop, \& A. E. Mazawi (Eds.), Pathologizing practices: The impact of deficit thinking on education (pp. 55-84). New York: Peter Lang.

Bishop, R., \& Berryman, M. (2006). Culture speaks. Wellington: Huia.

Bishop, R., \& Glynn, T. (1999). Culture counts: Changing power relations in education. Palmerston North: Dunmore Press.

Bishop, R., Berryman, M., Tiakiwai, S., \& Richardson, C. (2003). Te Kotahitanga Phase 1: The experiences of Year 9 and 10 Māori students in mainstream classrooms: Report to the Ministry of Education. Wellington: Learning Media.

Bouillion, L., \& Gomez, L. (2001). Connecting school and community with science learning: Real world problems and school-community partnerships as contextual scaffolds. Journal of Research in Science Teaching, 38(8), 878898.

Bourdieu, P. (1977). Outline of a theory of practice. Cambridge: Cambridge University Press.

Brenden, M. (2005). Funds of knowledge and team ethnography: Reciprocal approaches. In N. González, L. C. Moll, \& C. Amanti (Eds.), Funds of knowledge: Theorizing practices in households, communities, and classrooms (pp. 199-212). Mahwah, NJ: Lawrence Erlbaum. 
Briggs, C. L. (1986). Learning how to ask: A sociolinguistic appraisal of the role of the interview in social science research. Cambridge, UK: Cambridge University Press.

Browning-Aiken, A. (2005). Border crossings: Funds of knowledge within an immigrant household. In N. González, L. C. Moll, \& C. Amanti (Eds.), Funds of knowledge: Theorizing practices in households, communities, and classrooms (pp. 167-182). Mahwah, NJ: Lawrence Erlbaum.

Bryman, A. (1988). Quantity and quality in social research. London: Unwin Hyman.

Calabrese Barton, A., \& Tan, E. (2009). Funds of knowledge and discourses and hybrid space. Journal of Research in Science Teaching, 46(1), 50-73.

Camangian, P. (2010). Starting with self: Teaching autoethnography to foster critically caring literacies. Research in the Teaching of English, 45(2), 179204.

Carbone, P. C. (2012). Teachers' roles in facilitating novice writers from Generation 1.5. In B. Yoon \& H. K. Kim (Eds.), Teachers' roles in second language learning: Classroom applications of sociocultural theory (pp. 135-153). Charlotte, NC: Information Age.

Catsambis, S. (2001). Expanding knowledge of parental involvement in children's secondary education: Connections with high school seniors' academic success. Social Psychology of Education, 5, 149-177.

Civil, M., \& Bernier, E. (2006). Exploring images of parental participation in mathematics education: Challenges and possibilities. Mathematical Thinking and Learning, 8(3), 309-330.

Cohen, L., Manion, L., \& Morrison, K. (2007). Research methods in education ( $^{\text {th }}$ ed.). Abington, Oxon: Routledge.

Comber, B., \& Kamler, B. (2007). Getting out of deficit: Pedagogies of reconnection. Teaching Education, 15(3), 293-310.

Commission of Education in New Zealand. (1962). Report of the Commission on Education in New Zealand (The Currie Report). Wellington: Government Printer.

Conant, F., Rosebery, A., Warren, B., \& Hudicourt-Barnes, J. (2001). The sound of drums. In E. Mclntyre, A. Rosebery, \& N. González (Eds.), Classroom diversity: Connecting curriculum to students' lives (pp. 51-60). Portsmouth, $\mathrm{NH}$ : Heinemann.

Cook-Sather, A. (2002). Authorizing students' perspectives: Towards trust, dialogue, and change in education. Educational Researcher, 31(4), 3-14.

Cornelius-White, J. (2007). Learner-centered teacher-student relationships are effective: A meta-analysis. Review of Educational Research, 77(1), 113-143.

Cremin, T., Mottram, M., Collins, F., Powell, S., \& Drury, R. (2012). Building communities: Teachers researching literacy lives. Improving Schools, 15(2), 101-115.

Cullen, R. (2009). Culture, identity, and information privacy in the age of digital government. Online Information Review, 33(3), 405-421.

Darling-Hammond, L., \& Bransford, J. (2005). Preparing teachers for a changing world: What teachers should learn and be able to do. San Francisco, CA: Jossey-Bass. 
Davies, B., \& Harré, R. (1990). Positioning: The discursive production of selves. Journal for the Theory of Social Behavior, 20, 43-63.

Davis, H. A. (2003). Conceptualizing the role and influence of student-teacher relationships on children's social and cognitive development. Educational Psychologist, 38(4), 207-234.

De Shazer, S. (1982). Patterns of brief family therapy: An ecosystemic approach. New York: Guildford Press.

Delpit, L. (1995). Other people's children: Cultural conflict in the classroom. New York: The New Press.

Denny, S., Clark, T., \& Watson, P. (2004). The health of alternative education students compared to secondary school students: A New Zealand study. Journal of the New Zealand Medical Association, 117, 205. Retrieved from: http://www.nzma.org.nz/journal/117-1205/1147/ on 25 March 2010.

Denzin, N. K. (1989). The research act: $A$ theoretical introduction to sociological methods ( $3^{\text {rd }}$ ed.). Englewood Cliffs, NJ: Prentice-Hall.

Denzin, N. K. \& Lincoln, Y. S. (2000). Introduction: The discipline and practice of qualitative research. In N. K. Denzin \& Y. S. Lincoln (Eds.), Handbook of qualitative research $\left(2^{\text {nd }}\right.$ ed.). pp. 1-28. London: Sage.

Dodson, L., \& Schmalzbauer, L. (2010). Poor mothers and habits of hiding: Participatory methods in poverty research. In W. Luttrell (Ed.), Qualitative educational research: Readings in reflexive methodology and transformative practice (pp. 313-326). New York: Routledge.

Dworin, J. E. (2006). The family stories project: Using funds of knowledge for writing. The Reading Teacher, 59(6), 510-520.

Edwards, P. A., Pleasants, H. M., \& Franklin, S. H. (1999). A path to follow: Learning to listen to parents. Portsmouth, $\mathrm{NH}$ : Heinemann.

Eichelberger, R. T. (1989). Disciplined inquiry: Understanding and doing educational research. New York: Longman.

Eisenhart, M. (2001). Educational ethnography past, present, and future: Ideas to think with. Educational Researcher, 30(8), 16-27.

Erickson, F. (2007). Culture in society and in educational practices. In J. A. Banks \& C. A. M. Banks (Eds.), Multicultural education: Issues and perspectives $\left(6^{\text {th }}\right.$ ed., pp. 33-61). Hoboken, NJ: John Wiley.

Field, J. (2012). Learning from our lives. In P. Jarvis \& M. Watts (Eds.), The Routledge international handbook of learning (pp. 176-183). New York: Routledge.

Fielding, M. (2001). Beyond the rhetoric of student voice: New departures or new constraints in the transformation of $21^{\text {st }}$ century schooling. Forum, 43(2), 100 110.

Fielding, M. (2007). Beyond "voice": New roles, relations, and contexts in researching with young people. Discourse: Studies in the Cultural Politics of Education, 28(3), 301-310.

Fine, M., \& Weis, L. (2002). Writing the 'wrongs of fieldwork: Confronting our own research/writing dilemmas in urban ethnographies. In N. K. Denzin \& Y. S. Lincoln (Eds.), The qualitative inquiry reader (pp. 267-298). Thousand Oaks, CA: Sage. 
Fitts, S. (2009). Exploring third space in a dual-language setting: Opportunities and challenges. Journal of Latinos and Education, 8(2), 87-104.

Flick, U. (1998). The psychology of the social. Cambridge, UK: Cambridge University Press.

Flick, U. (2006). An introduction to qualitative research ( $3^{\text {rd }}$ ed.). London: Sage.

Flores Carmona, J., \& Delgado Bernal, D. (2012). Oral histories in the classroom. In C. E. Sleeter \& E. Soriano (Eds.), Creating solidarity across diverse communities (pp. 114-130). New York: Teachers College Press.

Floyd Tenery, M. (2005). La visita. In N. González, L. C. Moll, \& C. Amanti. (Eds.), Funds of knowledge: Theorizing practices in households, communities and classrooms (pp. 119-130). Mahwah, NJ: Lawrence Erlbaum.

Fordham, S., \& Ogbu, J. U. (1986). Black students' school success: Coping with the "burden of 'acting White." The Urban Review, 18, 176-206.

Fox-Turnbull, W. (2012, June). Funds of knowledge in technology education. Paper presented at the PATT 26 Conference: Technology Education in the $21^{\text {st }}$ Century, Stockholm, Sweden. Retrieved May 21, 2013, from www.ep.liu.se/ecp/073/021/ecp12073021.pdf.

Fraser-Abder, P., Doria, J. A., Yang, J., \& De Jesus, A. (2010). Using funds of knowledge in an ethnically concentrated environment to teach nutrition. Science Activities, 47, 141-150.

Gall, M. D., Gall, J. P., \& Borg, W. R. (2007). Educational research: An introduction $\left(8^{\text {th }}\right.$ ed.). New York: Pearson Education.

Gay, G. (2010). Culturally responsive teaching: Theory, research, and practice. New York: Teachers College Press.

Gee, J. (1996). Social linguistics and literacies: ideology in discourses. London: Falmer Press.

Gee, J. P. (1999). An introduction to discourse analysis: Theory and method. London: Routledge.

González, N. (1995). Processual approaches to multicultural education. Journal of Applied Behavioral Science, 31(2), 234-244.

González, N. (2005). The hybridity of funds of knowledge. In N. González, L. C. Moll, \& C. Amanti. (Eds.), Funds of knowledge: Theorizing practices in households, communities and classrooms (pp. 29-46). Mahwah, NJ: Lawrence Erlbaum.

González, N., \& Moll, L. (2002). Cruzando el Puente: Building bridges to funds of knowledge. Educational Policy, 16, 623-641.

González, N., Andrade, R., Civil, M., \& Moll, L. (2001). Bridging funds of distributed knowledge: Creating zones of practices in mathematics. Journal of Education for Students Placed at Risk, 6(1\&2), 115-132.

González, N., Moll, L., \& Amanti, C. (2005). Introduction: Theorizing practices. In N. González, L. C. Moll, \& C. Amanti. (Eds.), Funds of knowledge: Theorizing practices in households, communities and classrooms (pp. 1-28). Mahwah, NJ: Lawrence Erlbaum.

González, N., Moll, L., Floyd Tenery, M., Rivera, A., Rendon, P., Gonzales, R., \& Amanti, C. (1995). Funds of knowledge for teaching in Latino households. Urban Education, 29(1), 443-470. 
González, N., Wyman, L., \& O'Connor, B. (2011). The past, present, and future of 'funds of knowledge'. In B. A. U. Levinson \& M. Pollock (Eds.), A companion to the anthropology of education (pp. 481-494). Chichester, West Sussex: Wiley-Blackwell.

Good, T. L., \& Brophy, J. E. (1970). Teachers' communication of differential expectations for children's classroom performance: Some behavioral data. Journal of Educational Psychology, 61, 365-374.

Grant, C. A., \& Sleeter, C. E. (2007). Doing multicultural education for achievement and equity. New York: Routledge.

Gregory, A., Skiba, R. J., \& Noguera, P. A. (2010). The achievement gap and the discipline gap: Two sides of the same coin? Educational Researcher, 39, 5968.

Grootveld, C. M. (2013). Critical perspectives on the transformative potential of higher education in Aotearoa New Zealand, unpublished doctoral dissertation, Victoria University of Wellington, NZ.

Grunbaum, N. N. (2007). Identification of ambiguity in the case study research typology: What is a unit of analysis? Qualitative Market Research: An International Journal, 10(1), 78-87.

Grundy, S. (1987). Curriculum: Product or praxis. Lewes: Falmer.

Guba, E. G., \& Lincoln, Y. S. (1989). Fourth generation evaluation. Newbury Park, CA: Sage.

Guskey, T. R. (1988). Teacher efficacy, self-concept, and attitudes towards the implementation of instructional innovation. Teaching and Teacher Education, 4(1), 63-69.

Gutiérrez, K., \& Rogoff, B. (2003). Cultural ways of learning: Individual traits or repertoires of practice. Educational Researcher, 32(5), 19-25.

Gutiérrez, P. S. (2002). In search of bedrock: Organizing for success with diverse needs children in the classroom. Journal of Latinos and Education, 1(1), 4964.

Hager, P. (2012). Informal learning. In P. Jarvis \& M. Watts (Eds.), The Routledge international handbook of learning (pp. 207-215). New York: Routledge.

Hall, C. (2007). Evaluation as a method of educational inquiry. School of Education Victoria University of Wellington (class handout).

Hammond, L. (2001). Notes from California: An anthropological approach to urban science education for minority language families. Journal of Research in Science Teaching, 38(9), 983-999.

Hanson, M. J. (1992). Ethnic, cultural, and language diversity in intervention settings. In E. W. Lynch \& M. J. Hanson (Eds.), Developing cross-cultural competence (pp. 3-18). Baltimore: Paul H. Brookes.

Harding, S. (1993). Rethinking standpoint epistemology: What is "strong objectivity"? In L. Alcoff \& E. Potter (Eds.), Feminist epistemologies (pp. 49-82). New York: Routledge.

Hardy, L. (2006). The future of teaching. American School Board Journal, 193(11), 17.

Harker, R., \& Nash, R. (1990). Cultural reproduction and school achievement: A case for kura kaupapa Māori. ACCESS, 9(2), 26-39. 
Harry, B., Klingner, J. K., \& Hart, J. (2005). African American families under fire: Ethnographic views of family strengths. Remedial and Special Education, 26(2), 101-112

Hattam, R., \& Prosser, B. (2008). Unsettling deficit views of students and their communities. The Australian Educational Researcher, 35(2), 89-106.

Hawk, K., \& Hill, J. (2000). Making a difference in the classroom: effective teaching practice in low decile, multicultural schools. (Report prepared for the Ministry of Education and the AIMHI Forum). Retrieved May 20, 2013, from http://www.aimhi.ac.nz/downloads/makingadifference3311.pdf

Hawk, K., Cowley, E. T., Hill, J., \& Sutherland, S. (2002). The importance of the teacher/student relationship for Māori and Pasifika students. set, 3, 44-49.

Heath, S. B. (1983). Ways with words. Cambridge, UK: Cambridge University Press.

Heath, S. B. (2004). Risks, rules and roles. In A. N. Perret-Clermont, C. Pontecorvo, L. B. Resnick, T. Zittoun, \& B. Burge (Eds.), Joining society (pp. 41-70). Cambridge, UK: Cambridge University Press.

Henderson, D., \& Zipin, L. (2010). Bringing clay to life: Developing student literacy through clay-animation artwork that tells life-based stories. In B. Lucas, B. Prosser, \& A. Reid (Eds.), Connecting lives and learning: Teacher inquiry in the middle years of schooling (pp. 20-41). Adelaide: Wakefield Press, Hawke Research Institute Series.

Hensley, M. (2005). Empowering parents of multicultural backgrounds. In N. González, L. C. Moll, \& C. Amanti (Eds.), Funds of knowledge: Theorizing practices in households, communities, and classrooms (pp. 143-152). Mahwah, NJ: Lawrence Erlbaum.

Hogg, L. (2008). Transcending monocultural life experiences for Pākehā trainee teachers. New Zealand Annual Review of Education, 18, 89-106.

Hogg, L. (2011). Funds of knowledge: An investigation of coherence within the literature. Teaching and Teacher Education, 27(3), 666-677.

Hogg, L. (2012). Funds of knowledge: An examination of theoretical frameworks. New Zealand Annual Review of Education, 41, 47-76.

Hogg, L. (2013a, April). Preferred approaches to identify minority ethnicity students' funds of knowledge: A New Zealand case study. Paper presented at the AERA Annual Meeting, San Francisco, USA.

Hogg, L. (2013b, April). Collaborative team-based research involving students, parents, and teachers: Challenges and opportunities identified in a New Zealand case study. Paper presented at the AERA Annual Meeting, San Francisco, USA.

Hornby, G. (2000). Improving parental involvement. London: Cassell.

Hughes, M., \& Greenhough, P. (2006). Boxes, bags and videotape: Enhancing home-school communication through knowledge exchange activities. Educational Review, 58(4), 471-487.

Hughes, M., \& Pollard, A. (2006). Home-school knowledge exchange in context. Educational Review, 58(4), 385-395.

Hullena, T., \& Hullena, V. (2010). Student-teacher relationships: A pathway for atrisk youth. In V. A. Green \& S. Cherrington (Eds.), Delving into diversity: An international exploration of issues of diversity in education (pp. 9-20). New York: Nova Science. 
Hycner, R. H. (1985). Some guidelines for the phenomenological analysis of interview data. Human Studies, 8, 279-303.

Irvine, J. J. (2003). Educating teachers for diversity: Seeing with a cultural eye. New York: Teachers College Press.

Irvine, J. J., \& York, D. E. (1993). Teacher perspectives: Why do African American, Hispanic, and Vietnamese children fail? In S. W. Rothstein (Ed.), Handbook of schooling in urban America (pp. 161-173). Westport, CT: Greenwood Press.

Janesick, V. J. (2003). The choreography of qualitative research design: Minuets, improvisations, and crystallization. In N. K. Denzin \& Y. S. Lincoln (Eds.), Strategies of qualitative inquiry $\left(2^{\text {nd }}\right.$ ed., pp. 379-399). Thousand Oaks, CA: Sage.

Jorgensen, D. L. (1989). Participant observation: A methodology for human studies. Newbury Park, CA: Sage.

Joyce, B., \& Showers, B. (1980). Improving inservice training: The messages of research. Educational Leadership, 37(5), 379-385.

Kahn, L. H., \& Civil, M. (2001). Unearthing the mathematics of a classroom garden. In E. Mclntyre, A. Rosebery \& N. González (Eds.), Classroom diversity: Connecting curriculum to students' lives (pp. 37-50). Portsmouth, $\mathrm{NH}$ : Heinemann.

KewelRamani, A., Gilbertson, L., Fox, M., \& Provasnik, S. (2007). Status and trends in the education of racial and ethnic minorities (NCES 2007-039). Washington, DC: National Centre for Educational Statistics, Institute of Education Sciences, U.S. Department of Education. Retrieved May 27, 2010, from http://nces.ed.gov/pubs2007/2007039.pdf

King, A. R. (1967). The school at Mopass. New York: Holt, Reinhart, \& Winston.

King, J. E. (2004). Dysconscious racism: Ideology, identity, and the mis-education of teachers. In G. Ladson-Billings \& D. Gillborn (Eds.), The Routledge-Falmer reader in multicultural education (pp. 71-83). London: Routledge-Falmer.

Klenowski, V. (2009). Australian indigenous students: Addressing equity issues in assessment. Teaching Education, 20(1), 77-93.

Kohere, R. (1997). Nga kōrero a Reweti Kohere Mā: He kupu, he pepeha, he whakataukī. Wellington: Victoria University Press. Retrieved October 23, 2013, from http://nzetc.victoria.ac.nz/tm/scholarly/tei-TeoNgak-_N79717.html.

Kumpulainen, K., \& Lipponen, L. (2012). Harnessing funds of knowledge in dialogic inquiry across formal and informal learning environments. In $\mathrm{P}$. Jarvis with $\mathrm{M}$. Watts. (Eds.), The Routledge international handbook of learning (pp. 112125). New York: Routledge.

Lange, D. (1988). Tomorrow's schools: The reform of educational administration in New Zealand. Wellington: Ministry of Education.

Lave, J., \& Wenger, E. (1991). Situated learning: Legitimate peripheral participation. New York: Cambridge University Press.

Lee, C. D. (1998). Culturally responsive pedagogy and performance-based assessment. The Journal of Negro Education, 67(3), 268-279.

Lee, C. D. (2001). Is October Brown Chinese? A cultural modeling activity system for underachieving students. American Educational Research Journal, 38(1), 97-141. 
Lee, T. S., \& Qiujada Cerecer, P. D. (2010). (Re)claiming native youth knowledge: Engaging in socio-culturally responsive teaching and relationships. Multicultural Perspectives, 12(4), 199-205.

Levin, B. (2000). Putting students at the centre in education reform. Journal of Educational Change, 1, 155-172.

Lewis, O. (1966). The culture of poverty. Scientific American, 215(4), 19-25.

Lincoln, Y. S. \& Guba, E. G. (2000). Paradigmatic controversies, contradictions, and emerging confluences. In N. K. Denzin \& Y. S. Lincoln, (Eds.), Handbook of qualitative research $\left(2^{\text {nd }} e d, p p .163-188\right)$. London: Sage.

Lomax, J. W. (2007). Building the foundation. Psychiatry, 70(3), 209-214.

Macias, A. (2013, April). Working-class Latino students and the influence of funds of knowledge on their school engagement. Paper presented at the AERA Annual Meeting, San Francisco, USA.

Maher, S., Epaloose, G., \& Tharp, R. (2001). Connecting cultural traditions. In E. Mclntyre, A. Rosebery \& N. González (Eds.), Classroom diversity: Connecting curriculum to students' lives (pp. 14-26). Portsmouth, $\mathrm{NH}$ : Heinemann.

Mallard, T. (2003). NCEA statistics a milestone for learning. Retrieved March 25, 2010. from http://www.beehive.govt.nz/node/16738.

Mapp, K. L. (2003). Having their say: Parents describe why and how they are engaged in their children's learning. The School Community Journal, 13, 3564.

Marshall, E., \& Toohey, K. (2010). Representing family: Community funds of knowledge, bilingualism, and multimodality. Harvard Educational Review, 80(2), 221-288.

Martin-Jones, M., \& Saxena, M. (2003). Bilingual resources and 'Funds of Knowledge' for teaching and learning in multi-ethnic classrooms in Britain. In A. Creese \& P. Martin (Eds.), Multilingual classroom ecologies: Interrelationships, interactions and ideologies (pp. 107-122). Clevedon, UK: Multilingual Matters.

McIntyre, E., Kyle, D., Moore, G., Sweazy, R. A., \& Greer, S. (2001). Linking home and school through family visits. Language Arts, 78(3), 264-272.

Mercado, C. (2005). Reflections on the study of households in New York City and Long Island: A different route, a common destination. In N. González, L. C. Moll, \& C. Amanti (Eds.), Funds of knowledge: Theorizing practices in households, communities, and classrooms (pp. 233-256). Mahwah, NJ: Lawrence Erlbaum.

Mertens. D. (2005). Research and evaluation in psychology: Integrating diversity with quantitative, qualitative, and mixed methods $\left(2^{\text {nd }}\right.$ ed.). Thousand Oaks, CA: Sage.

Messing, J. (2005). Social reconstructions of schooling: Teacher evaluations of what they learned from participation in the Funds of Knowledge Project. In N. González, L. C. Moll, \& C. Amanti (Eds.), Funds of knowledge: Theorizing practices in households, communities, and classrooms (pp. 183-194). Mahwah, NJ: Lawrence Erlbaum.

Michaels, S. (1985). Hearing the connections in children's oral and written discourse. Journal of Education, 167, 36-56. 
Miles, M., \& Huberman, M. (1984). Qualitative data analysis. Beverley Hills, CA: Sage.

Miller, A. (1994). Staff culture, boundary maintenance and successful behavioural interventions in primary schools. Research Papers in Education, 9(1), 31-51.

Miller, A. (1996). Pupil behaviour and teacher culture. London: Cassell.

Ministry of Education. (2002a). Briefing for the incoming Minister for Education 2002. Wellington: Learning Media.

Ministry of Education. (2002b). Alternative education statistics, December 2002. Wellington: Ministry of Education.

Ministry of Education. (2005). Ministry of Education suspension reduction initiative August 05. Retrieved March 25, 2010, from www.tki.org.nz/r/governance/.../suspensions reduction summary.doc.

Ministry of Education. (2007a). A report on New Zealand student engagement 2006. Retrieved 25 March, 2010, from www.educationcounts.govt.nz > ... > Student Engagement.

Ministry of Education. (2007b). Ka Hikitia - Managing for success: The Māori education strategy 2008-2012. Retrieved May 20, 2013, from http://www.minedu.govt.nz/theMinistry/PolicyAndStrategy/KaHikitia.aspx

Ministry of Education. (2007c). The New Zealand curriculum. Wellington: Learning Media.

Ministry of Education. (2010). Ngā Haeata Mātauranga: The annual report on Māori education 2008/2009. Wellington: Ministry of Education.

Ministry of Education. (n.d.). Deciles. Retrieved from: http://www.minedu.govt.nz/NZEducation/EducationPolicies/Schools/SchoolO perations/Resourcing/OperationalFunding/Deciles.aspx on 14 June 2013.

Mitra, D. L. (2009). Collaborating with students: Building youth-adult partnerships in schools. American Journal of Education, 115(3), 407-436.

Moje, E. B., Ciechanowski, K. M., Kramer, K., Ellis, L., Carrillo, R., \& Collazo, T. (2004). Working toward third space in content area literacy: An examination of everyday funds of knowledge and Discourse. Reading Research Quarterly, 39(1), 38-70.

Moll, L. C. (1992). Bilingual classroom studies and community analysis: Some recent trends. Educational Researcher, 21(2), 20-24.

Moll, L. C. (2005). Reflection and possibilities. In N. González, L. C. Moll, \& C. Amanti (Eds.), Funds of knowledge: Theorizing practices in households, communities, and classrooms (pp. 275-287). Mahwah, NJ: Lawrence Erlbaum.

Moll, L. C., \& Cammarota, J. (2010). Cultivating new funds of knowledge through research and practice. In K. L. Dunsmore \& D. Fisher (Eds.), Bringing literacy home (pp. 289-305). Newark, DE: International Reading Association.

Moll, L. C., \& González, N. (2004). Engaging life: A funds of knowledge approach to multicultural education. In J. Banks \& C. McGee Banks (Eds.), Handbook of research on multicultural education ( $2^{\text {nd }}$ ed., pp. 699-715). New York: JosseyBass.

Moll, L. C., \& Greenberg, J. B. (1990). Creating zones of possibilities: Combining social contexts for instruction. In L. C. Moll (Ed.), Vygotsky and education: 
Instructional implications and applications of sociohistorical psychology (pp. 319-348). Cambridge, UK: Cambridge University Press.

Moll, L. C., \& Whitmore, K. F. (1993). Vygotsky in classroom practice: Moving from individual transmission to social interaction. In E. A. Forman, N. Mininck, \& C. A. Stone (Eds.), Contexts for learning: Sociocultural dynamics in children's development (pp. 19-42). New York: Oxford University Press.

Moll, L. C., Soto-Santiago, S. L., \& Schwartz, L. (2013). Funds of knowledge in changing communities. In K. Hall, T. Cremin, B. Comber, \& L. Moll (Eds.), International handbook of research on children's literacy, learning, and culture (pp. 172-183). Oxford, UK: John Wiley.

Moll, L. C., Vélez-lbáñez, C. G., \& Greenberg, J. (1989). Year one progress report: Community knowledge and classroom practice: Combining resources for literacy instruction. (IARP Subcontract No. L-10). Tucson, AZ: University of Arizona, College of Education and Bureau of Applied Research in Anthropology.

Moll, L. C., Vélez-lbáñez, C. G., Greenberg, J., Whitmore, K., Tapia, J., Saavedra, E., ... \& Andrade, R. (1990). Community knowledge and classroom practice: Combining resources for literacy instruction: Technical report. (OBEMLA Contract No. 300-87-0131). Tucson, AZ: University of Arizona College of Education and Bureau of Applied Research in Anthropology.

Moll, L., Amanti, C., Neff, D., \& González, N. (1992). Funds of knowledge for teaching: Using a qualitative approach to connect homes and classrooms. Theory into Practice, 31(2), 132-141.

Monzo, L., \& Rueda, R. (2003). Shaping education through diverse funds of knowledge: A look at one Latina paraeducator's lived experiences, beliefs, and teaching practice. Anthropology and Education Quarterly, 34(1), 72-95.

Narayan, K. (1993). How native is a "native" anthropologist? American Anthropologist, 95, 671-686.

Nelson, E., Christensen, K., \& Cleary, M. (2008). If we had mean-as (great) teachers we'd have a mean-as (great) school: Māori children in New Zealand. In E. Alerby \& E. Brown (Eds.), Voices from the margins: School experiences of refugee, migrant and indigenous children (pp. 129-143). Rotterdam: Sense.

New Zealand Teachers Council. (2009). Registered teacher criteria. Retrieved 21 May, 2013, from http://www.teacherscouncil.govt.nz/sites/default/files/registered-teachercriteria-eng-2009-10-28.pdf

Nias, J. (1985). Reference groups in primary teachers: Talking, listening, and identity. In S. J. Ball and I. F. Goodson (Eds.), Teachers' lives and careers (pp. 105-119). Lewes: Falmer.

Nicholson, T. (2000). Research focuses on student achievement and learning. New Zealand Education Review, 7(43), 3.

Nieto, S., \& Bode, P. (2008). Affirming diversity: the sociopolitical context of multicultural education ( $5^{\text {th }}$ ed.). New York: Pearson.

Noddings, N. (1984). Caring: A feminine approach to ethics and moral education. Berkeley, CA: University of California Press.

NZQA. (2010). Qualifications by year level and ethnicity. Retrieved 28 May 2010 from http://www.nzqa.govt.nz/qualifications/ssq/statistics/provider-selectedcrystalreport.do. 
Olmedo, I. M. (1997). Family oral histories for multicultural curriculum perspectives. Urban Education, 32, 45-62.

Ostermann, K. (2000). Students' need for belonging in the school community. Review of Educational Research, 70(3), 323-367.

Oughton, H. (2010). Funds of knowledge: A conceptual critique. Studies in the Education of Adults, 42(1), 63-78.

Ovsienko, H., \& Zipin, L. (2007). Making social justice curricular: Exploring ambivalences within teacher professional identity. In P. Jeffrey (Ed.), Proceedings of the 2006 Australian Association for Research in Education Annual Conference, Adelaide. Retrieved February 24, 2013, from http://www.aare.edu.au/06pap/ovs06836.pdf

Patterson, L., Baldwin, S., Gonzales, R., Guadarrama, I., \& Keith, L. (1999). A different approach to family involvement. Networks: An Online Journal for Teacher Research, 2(2). Retrieved February 26, 2013, from http://journals.library.wisc.edu/index.php/networks/article/view/65/68

Patterson, L., \& Baldwin, S. (2001). A different spin on parent involvement: Exploring funds of knowledge within a systems perspective. In W. Goodman (Ed.), Living and teaching in an unjust world: New perspectives on multicultural education (pp. 127-139). Portsmouth, NH: Heinemann.

Penetito, W. (2008). Place-based education: Catering for curriculum, culture, and community. New Zealand Annual Review of Education, 18, 5-29.

Penetito, W. (2010). What's Māori about Māori education? Wellington: Victoria University Press.

Peräkylä (2005). Analyzing talk and text. In N. K. Denzin \& Y. S. Lincoln (Eds.), The Sage handbook of qualitative research ( $3^{\text {rd }}$ ed., pp. 215-255). Thousand Oaks, CA: Sage.

Pirbhai-Illich, F. (2010). Aboriginal students engaging and struggling with critical multiliteracies. Journal of Adolescent and Adult Literacy, 54(4), 257-266.

Ranson, S. (2000). Recognizing the pedagogy of voice in a learning community. Educational Management and Administration, 28(3), 263-279.

Rice, A. K. (1969). Individual, group and inter-group processes. Human Relations, 22(6), 565-584.

Riley, R., Robinson, S., \& Elliott, E. (1993). School engagement and students at risk. New Jersey: National Center for Education Statistics.

Riojas-Cortez, M., \& Flores, B. B. (2009). Sin olvidar a los padres: Families collaborating within school and university partnerships. Journal of Latinos in Education, 8(3), 231-239.

Rios-Aguilar, C. (2010). Measuring funds of knowledge: Contributions to Latina/o academic and non-academic outcomes. Teachers College Record, 112(8), 2209-2257.

Rios-Aguilar, C., Kiyama, J. M., Gravitt, M., \& Moll, L. C. (2011). Funds of knowledge for the poor and forms of capital for the rich? A capital approach to examining funds of knowledge. Theory and Research in Education, 9(2), 163-184.

Rist, R. C. (1974). Race, policy, and schooling. Society, 12(1), 59-64.

Ritchie, J. (1992). Becoming bicultural. Wellington: Huia. 
Robson, C. (2011). Real world research (3rd ed.). West Sussex, UK: John Wiley.

Rodriguez, G. (2013). Power and agency in education: Exploring the pedagogical dimensions of funds of knowledge. Review of Research in Education, 37, 87120.

Rogers, R., Light, R., \& Curtis, L. (2004). "Anyone can be an expert in something": Exploring the complexity of discourse conflict and alignment for two fifth grade students. Journal of Literacy Research, 36, 177-210.

Rogoff, B. (1990). Apprenticeship in thinking: Cognitive development in social context. Oxford, UK: Oxford University Press.

Rosaldo, R. (1989). Culture and truth: The remaking of social analysis. Boston, MA: Beacon.

Rosebery, A., Mclntyre, E., \& González, N. (2001). Connecting students' cultures to instruction. In E. Mclntyre, A. Rosebery, \& N. González (Eds.), Classroom diversity: Connecting curriculum to students' lives (pp. 1-13). Portsmouth, $\mathrm{NH}$ : Heinemann.

Rosenfield, G. (1971). "Shut those thick lips!" A study of slum school failure. New York: Holt, Reinhart, \& Winston.

Rosenthal, R., \& Jacobson, L. (1966). Teachers' expectancies: Determinates of pupils' IQ gains. Psychological Reports, 19, 115-118.

Rowsell, J. (2006). Family literacy experiences: Creating reading and writing experiences that support classroom learning. Ontario, Canada: Pembroke.

Rudduck, J., \& Fielding, M. (2006). Student voice and the perils of popularity. Educational Review, 58(2), 219-231.

Rudduck, J. (2007). Student voice, student engagement, and school reform. In D. Thiessen \& A. Cook-Sather (Eds.), International handbook of student experience in elementary and secondary school (pp. 587-610). Dordrecht, The Netherlands: Springer.

Ryan, W. (1972). Blame the victim. New York: Vintage Books.

Sandoval-Taylor, P. (2005). Home is where the heart is: A funds of knowledgebased curriculum module. In In N. González, L. C. Moll, \& C. Amanti (Eds.), Funds of knowledge: Theorizing practices in households, communities, and classrooms (pp. 153-165). Mahwah, NJ: Lawrence Erlbaum.

Saubich, X., \& Esteban, M. (2011). Bringing funds of family knowledge to school. The Living Morocco Project. Multidisciplinary Journal of Educational Research, 1(1), 79-103.

Savage, C. (2010). Culturally responsive classroom management in New Zealand. In V. Green \& S. Cherrington (Eds.), Delving into diversity: An international exploration of issues of diversity in education (pp. 35-44). New York: Nova Science.

Schmuck, R. A. (2009). Defining action research. In R. A. Schmuck (Ed.), Practical action research: $A$ collection of articles ( $2^{\text {nd }}$ ed., pp. 1-2). Thousand Oaks, $\mathrm{CA}$ : Corwin.

Seiler, G. (2001). Reversing the "standard" direction: Science emerging from the lives of African American students. Journal of Research in Science Teaching, 38(9), 1000-1014. 
Sfard, A. (1998). On two metaphors for learning and the dangers of choosing just one. Educational Researcher, 27(2), 4-13.

Sleeter, C. E. (2005). Un-standardizing curriculum: Multicultural teaching in the standards-based classroom. New York: Teachers College Press.

Sleeter, C. (2008). Preparing white teachers for diverse students. In M. CochranSmith, S. Feiman-Nemser, D. J. Mclntyre, \& K. E. Demers (Eds.), Handbook of research on teacher education: Enduring questions in changing contexts ( $3^{\text {rd }}$ ed., pp. 559-582). New York: Routledge, Taylor \& Francis Group and the Association of Teacher Education.

Sleeter, C. (2011). The quest for social justice in the education of minoritized students. In C. Sleeter (Ed.), Professional development for culturally responsive and relationship-based pedagogy (pp. 1-21). New York: Peter Lang.

Smith, G. H. (1995). Whakaono whānau: New formations of whānau as an innovative intervention into Māori cultural and educational crises. He Pukenga Kōrero, 1(1), 18-36.

Smith, L. T. (2005). On tricky ground: Researching the native in the age of uncertainty. In N. K. Denzin \& Y. S. Lincoln, (Eds.), The Sage handbook of qualitative research (3rd ed.. pp. 85-107). Thousand Oaks, CA: Sage.

Smythe, S., \& Toohey, K. (2009). Investigating sociohistorical contexts and practices through a community scan: A Canadian Punjabi-Sikh example. Language and Education, 23(1), 37-57.

Spiller, L. T. (2013). Teachers' misunderstandings that affect the learning of their Pasifika students. (MEd thesis). Victoria University of Wellington, NZ.

Spindler, G., \& Spindler, L. (1983). Review essay: The "case studies in education and culture: From cradle to grave". Anthropology and Education Quarterly, 14(2), 73-80.

Spradley, J. P. (1980). Participant observation. New York: Rinehart \& Winston.

Stainback, S., \& Stainback, W. (1988). Understanding and conducting qualitative research. Dubuque, IA: Kendall/Hunt.

Stake, R. (1995). The art of case study research. Thousand Oaks, CA: Sage.

Stake, R. (2000). Case studies. In N. K. Denzin \& Y. S. Lincoln, (Eds.), Handbook of qualitative research, $\left(2^{\text {nd }}\right.$ ed., pp. 435-454). London: Sage.

Sugarman, S. (2010). Seeing past fences: Finding funds of knowledge for ethical teaching. The New Educator, 6(2), 96-117.

Tappan, M. B. (1998). Sociocultural psychology and caring pedagogy: Exploring Vygotsky's 'hidden curriculum." Educational Psychologist, 33(1), 23-33.

Tharp, R., \& Gallimore, R. (1993). Rousing minds to life: Teaching, learning and schooling in social context. New York: Cambridge University Press.

Thomson, P., \& Hall, C. (2008). Opportunities missed and/or thwarted? Funds of knowledge meet the English national curriculum. Curriculum Journal, 19(2), 87-103.

Thomson, P., Hall, C., \& Russell, L. (2006). An arts project failed, censored or,,,? A critical incident approach to artist-school partnerships. Changing English, 13(1), 29-44. 
Timperley H. S., \& Robinson, V. M. J. (2002). Achieving school improvement through challenging and changing teachers' schema. Journal of Educational Change, 2, 281-300.

Ting-Toomey, S., \& Oetzel, J. G. (2001). Managing intercultural conflict effectively. Thousand Oaks, CA: Sage.

Upadhyay, B. R. (2005). Using students' lived experiences in an urban science classroom: An elementary school teacher's thinking. Science Education, 90(1), 94-110.

Upadhyay, B. R. (2009). Teaching science for empowerment in an urban classroom: A case study of a Hmong teacher. Equity and Excellence in Education, 42(2), 217-232.

Valencia, R. R. (2010). Dismantling contemporary deficit thinking: Educational thought and practice. New York: Routledge.

Valli, L. (1996). Learning to teach in cross-cultural settings. In F. A. Rios (Ed.), Teacher thinking in cultural contexts (pp. 282-307). New York: State University of New York Press.

Varelas, M., \& Pappas, C. C. (2006). Intertextuality in read-alouds of integrated science-literacy units in urban primary classrooms: Opportunities for the development of thought and language. Cognition and instruction, 24(2), 211259.

Vélez-lbáñez, C. G., \& Greenberg, J. B. (1992). Formation and transformation of funds of knowledge among U.S.-Mexican households. Anthropology and Education Quarterly, 23(4), 313-335.

Vélez-Ibáñez, C. G. (1988). Networks of exchange among Mexicans in the U.S. and Mexico: Local level mediating responses to national and international transformations. Urban Anthropology, 17(1), 27-51.

Vertovec, S. (Ed.). (2009). Migration: Critical concepts in the social sciences. London: Routledge.

Vygotsky, L. (1978). Mind in society. Cambridge, MA: Harvard University Press.

Vygotsky, L. (1986). Thought and language. Cambridge, MA: MIT Press.

Walliman, N. (2005). Your research project ( $2^{\text {nd }}$ ed.). London: Sage.

Ward, M. C. (1971). Them children. New York: Holt, Reinhart, \& Winston.

Warren, B., \& Rosebery, A. (1996). "This question is just too, too easy!": Students perspectives on accountability in science. In L. Schauble \& R. Glaser (Eds.), Innovations in learning: New environments for education (pp. 97-125). Mahwah, NJ: Erlbaum.

Wentzel, K. R. (1997). Student motivation in middle school: The role of perceived pedagogical caring. Journal of Educational Psychology, 89(3), 411-419.

Wink, J., \& Putney, L. (2002). A vision of Vygotsky. Boston, MA: Allyn \& Bacon.

Winn Tutwiler, S. J. (2005). Teachers as collaborative partners: Working with diverse families and communities. Mahwah, $\mathrm{NJ}$ : Lawrence Erlbaum.

Wolcott, H. (1967). A Kwakuitl village and school. New York: Holt, Reinhart, \& Winston. 
Wolcott, H. F. (1992). Posturing in qualitative inquiry. In M. D. LeCompte, W. L. Millroy, \& J. Preissle (Eds.), Handbook of qualitative research in education (pp. 3-52). San Diego, CA: Academic Press.

Wolf, E. (1966). Peasants. Englewood Cliffs, NJ: Prentice-Hall.

Woodrow, K. (2013, April). Bringin' it: Funds of knowledge and the social organization of the middle school classroom. Paper presented at the AERA Annual Meeting, San Francisco, USA.

Yin, R. K. (2009). Case study research: Design and methods ( $4^{\text {th }}$ ed.). Thousand Oaks, CA: Sage.

Yoon, B. (2012). The teacher's cultural inclusivity and English language learners' participation in language and literacy activities. In B. Yoon \& H. K. Kim (Eds.), Teachers' roles in second language learning: Classroom applications of sociocultural theory (pp. 155-169). Charlotte, NC: Information Age.

Yosso, T. J. (2005). Whose culture has capital? A critical race theory discussion of community cultural wealth. Race, Ethnicity, and Education, 8(1), 69-91.

Zipin, L. (2009). Dark funds of knowledge, deep funds of pedagogy: Exploring boundaries between lifeworlds and schools. Discourse: Studies in the Cultural Politics of Education, 30(3), 317-331.

Zipin, L., Sellar, S., \& Hattam, R. (2012). Countering and exceeding 'capital': A 'funds of knowledge' approach to re-imagining community. Discourse: Studies in the Cultural Politics of Education, 33(2), 179-192.

Zuber-Skerrit, O. (1996). Introduction. In O. Zuber-Skerrit (Ed.), New directions in action research (pp. 3-9). London: Falmer. 


\section{Appendices}




\section{Appendix A: Confirmation of ethical approval for the research}

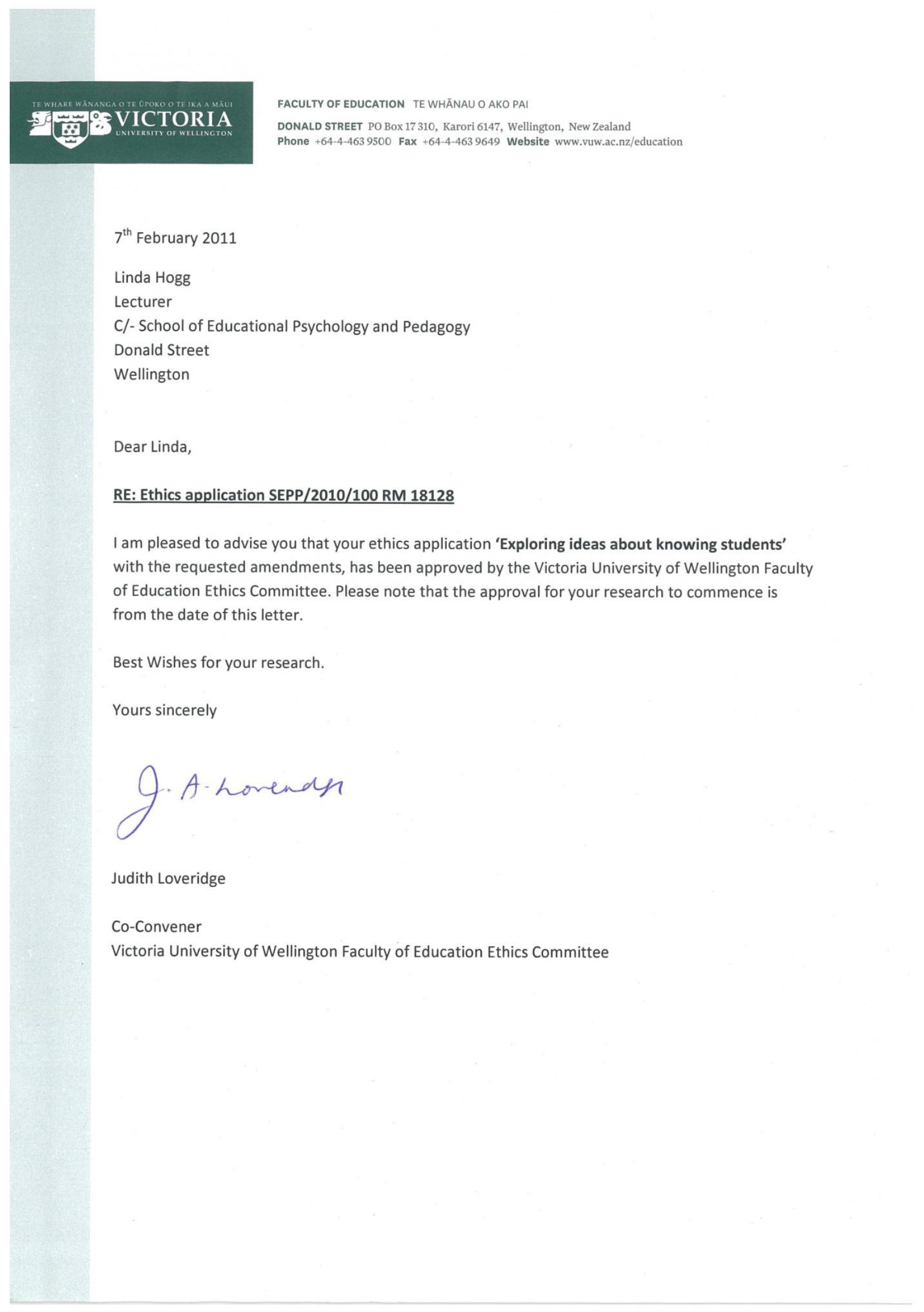


Appendix B: Completion of research events by participants, by team

\begin{tabular}{|c|c|c|c|c|c|c|c|c|c|c|c|c|c|c|c|c|c|c|}
\hline & Name & & Hui 1 & $\begin{array}{c}\text { Hui } 1 \\
\text { Date/ time }\end{array}$ & $\begin{array}{c}\mathrm{H} 1 \\
\mathrm{R}\end{array}$ & $\begin{array}{c}\text { Hui } 2 \\
\text { Date/ } \\
\text { time }\end{array}$ & $\begin{array}{c}\mathrm{H} 2 \\
\mathrm{R}\end{array}$ & $\begin{array}{c}\mathrm{FG} \\
\text { Date/ } \\
\text { time }\end{array}$ & $\begin{array}{c}\mathrm{FG} \\
\mathrm{R}\end{array}$ & $\begin{array}{c}\text { Hui } 3 \\
\text { Date/ } \\
\text { time }\end{array}$ & $\begin{array}{c}\mathrm{H} 3 \\
\mathrm{R}\end{array}$ & $\begin{array}{c}\mathrm{FG}(\mathrm{T}) \\
\text { Date/ } \\
\text { time }\end{array}$ & $\begin{array}{c}\text { Hui } 4 \\
\text { Date/ } \\
\text { time }\end{array}$ & $\begin{array}{c}\mathrm{H} 4 \\
\mathrm{R}\end{array}$ & $\begin{array}{c}\mathrm{FG} \\
\text { Date/ } \\
\text { time }\end{array}$ & FG R & $\begin{array}{l}\text { Ind } \\
\text { Int } \\
\text { Date/ } \\
\text { time }\end{array}$ & $\begin{array}{l}\text { II } \\
\mathrm{R}\end{array}$ \\
\hline \multirow[t]{5}{*}{ A } & Lizzie & $\mathrm{T}$ & $\mathrm{T}$ & $\begin{array}{l}\text { M13/6 } \\
12.20 \mathrm{p}\end{array}$ & 0 & $\begin{array}{l}\text { W22/6 } \\
5 p\end{array}$ & 0 & $\begin{array}{l}\text { M 8/8 } \\
12.40 p\end{array}$ & 0 & Th1/9 5p & 0 & $\begin{array}{l}\text { Fr23/9 } \\
12.40 p\end{array}$ & $\begin{array}{l}\text { T 1/11 } \\
5 p\end{array}$ & 0 & $\begin{array}{l}\text { Th } 10 / 11 \\
2 p\end{array}$ & 0 & $\begin{array}{l}\text { T 17/11 } \\
2.15 p\end{array}$ & $1 \mathrm{a}$ \\
\hline & Sonny Bill & $S$ & $S$ & T21/6 3p & $1 a$ & $\begin{array}{l}\text { W22/6 } \\
5 p\end{array}$ & 0 & $\begin{array}{l}\mathrm{F} 24 / 6 \\
3 p\end{array}$ & 0 & Th1/9 5p & 0 & - & $\begin{array}{l}T 1 / 11 \\
5 p\end{array}$ & 0 & Abs, b & & $\begin{array}{l}\text { W 16/11 } \\
3 p\end{array}$ & 1c \\
\hline & Thor & $S$ & $S$ & T21/6 3p & $1 d$ & $\begin{array}{l}\text { W22/6 } \\
5 p\end{array}$ & 0 & $\begin{array}{l}\mathrm{M} 11 / 7 \\
3 p\end{array}$ & $1 \mathrm{~b}$ & Th1/9 5p & 0 & - & $\begin{array}{l}T 1 / 11 \\
5 p\end{array}$ & 0 & Abs, $d$ & & $\begin{array}{l}\text { T } 15 / 11 \\
1.20 p\end{array}$ & 0 \\
\hline & Ses & $P$ & $P$ & $M 13 / 65 p$ & 0 & $\begin{array}{l}\text { W22/6 } \\
5 p\end{array}$ & 0 & $\begin{array}{l}\text { M11/7 } \\
5 p\end{array}$ & $1 b$ & Th1/9 5p & 0 & - & $\begin{array}{l}\text { T 1/11 } \\
5 p\end{array}$ & 0 & Abs, b & & $\begin{array}{l}\text { T 15/11 } \\
4.30 p\end{array}$ & 0 \\
\hline & Carol & $P$ & abs & abs & $1 d$ & $\begin{array}{l}\text { W22/6 } \\
5 p\end{array}$ & 0 & abs & $2 d, d$ & WD s & - & - & - & - & - & - & - & \\
\hline \multirow[t]{5}{*}{ B } & Kate & $\mathrm{T}$ & $\mathrm{T}$ & $\begin{array}{l}M 13 / 6 \\
12.20 p\end{array}$ & 0 & $\begin{array}{l}M 20 / 6 \\
5 p\end{array}$ & 0 & $\begin{array}{l}M 8 / 8 \\
12.40 p\end{array}$ & & M29/8 5p & 0 & $\begin{array}{l}\text { Fr23/9 } \\
12.40 p\end{array}$ & $\begin{array}{l}M 31 / 10 \\
5 p\end{array}$ & 0 & $\begin{array}{l}\text { Th } 10 / 11 \\
2 p\end{array}$ & 0 & $\begin{array}{l}\text { T 15/11 } \\
11.20 \mathrm{a}\end{array}$ & 0 \\
\hline & Troy & $S$ & Mg1 & M20/6 3p & $1 d$ & Absent & & - & & M29/8 5p & 0 & - & Abs (sports) & 0 & Abs, $d$ & & $\begin{array}{l}\text { T 1/11 } \\
3 p\end{array}$ & 0 \\
\hline & Tash & $P$ & $P$ & $M 13 / 65 p$ & 0 & $\begin{array}{l}M 20 / 6 \\
5 p\end{array}$ & 0 & $\begin{array}{l}M 27 / 6 \\
5 p\end{array}$ & 0 & $M 29 / 85 p$ & 0 & - & $\begin{array}{l}M 31 / 10 \\
5 p\end{array}$ & 0 & $\begin{array}{l}M 7 / 11 \\
5 p\end{array}$ & 0 & $\begin{array}{l}\text { W 16/11 } \\
4.30 p\end{array}$ & 0 \\
\hline & Catherine & $\mathrm{P}$ & $\mathrm{P}$ & $M 13 / 65 p$ & 0 & $\begin{array}{l}\text { M20/6 } \\
5 p\end{array}$ & 0 & $\begin{array}{l}M 27 / 6 \\
5 p\end{array}$ & 0 & M29/8 5p & 0 & - & $\begin{array}{l}M 31 / 10 \\
5 p\end{array}$ & 0 & $\begin{array}{l}M 7 / 11 \\
5 p\end{array}$ & 0 & $\begin{array}{l}\text { F 25/11 } \\
5.30 p\end{array}$ & $1 b$ \\
\hline & Dan & $S$ & Mg1 & M20/6 3p & $1 d$ & Absent & & - & & M29/8 5p & 0 & - & $\begin{array}{l}M 31 / 10 \\
5 p\end{array}$ & 0 & Abs, $d$ & & $\begin{array}{l}F 25 / 11 \\
2 p\end{array}$ & 0 \\
\hline \multirow[t]{5}{*}{ C } & Pearl & $P$ & $\mathrm{Mg} 1$ & $\begin{array}{l}\mathrm{M} 20 / 6 \\
3 p\end{array}$ & $1 \mathrm{c}$ & absent & $2 e$ & $\begin{array}{l}M 11 / 7 \\
5 p\end{array}$ & $1 d$ & Th8/9 5.30p & 0 & - & $\begin{array}{l}\text { W 12/10 } \\
11 a\end{array}$ & 0 & $\begin{array}{l}M 7 / 11 \\
5 p\end{array}$ & 0 & $\begin{array}{l}\text { M 14/11 } \\
4.30 p\end{array}$ & 0 \\
\hline & Jacob & $S$ & Mg1 & $\begin{array}{l}\text { M20/6 } \\
3 p\end{array}$ & $1 \mathrm{c}$ & $\begin{array}{l}\text { Th7/7 } \\
5.30 p\end{array}$ & $2 e$ & $\begin{array}{l}\mathrm{M} 11 / 7 \\
3 p\end{array}$ & $1 d$ & Th8/9 5.30p & 0 & - & $\begin{array}{l}\text { W 12/10 } \\
11 a\end{array}$ & 0 & $\begin{array}{l}\text { Abs, a (stand } \\
\text { down) }\end{array}$ & & $\begin{array}{l}M 14 / 11 \\
3 p\end{array}$ & 0 \\
\hline & Georgia & $\mathrm{T}$ & $\mathrm{T}$ & $\begin{array}{l}\mathrm{M} 13 / 6 \\
12.20 \mathrm{p}\end{array}$ & 0 & $\begin{array}{l}\text { Th7/7 } \\
5.30 p\end{array}$ & $2 e$ & $\begin{array}{l}\text { M 8/8 } \\
12.40 p\end{array}$ & 0 & Th8/9 5.30p & 0 & $\begin{array}{l}\text { abs } \\
\text { (sick) }\end{array}$ & $\begin{array}{l}\text { W 12/10 } \\
11 a\end{array}$ & 0 & $\begin{array}{l}\text { Th } 10 / 11 \\
2 p\end{array}$ & 0 & 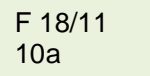 & 0 \\
\hline & Briar & $S$ & $\mathrm{Mp}$ & $\begin{array}{l}\text { W6/7 } \\
1.25 p\end{array}$ & 0 & $\begin{array}{l}\text { Th7/7 } \\
5.30 p\end{array}$ & 0 & $\begin{array}{l}M 11 / 7 \\
3 p\end{array}$ & 0 & Th8/9 5.30p & 0 & - & $\begin{array}{l}\text { W 12/10 } \\
11 a\end{array}$ & 0 & $\begin{array}{l}M 7 / 11 \\
3 p\end{array}$ & 0 & $\begin{array}{l}\text { Th } 17 / 11 \\
1.20 p\end{array}$ & 0 \\
\hline & Sandy & $\mathrm{P}$ & $\mathrm{Mp}$ & $\begin{array}{l}W 6 / 7 \\
1.25 p\end{array}$ & 0 & $\begin{array}{l}\text { Th7/7 } \\
5.30 p\end{array}$ & 0 & $\begin{array}{l}M 11 / 7 \\
5 p\end{array}$ & 0 & Th8/9 5.30p & 0 & - & $\begin{array}{l}\text { W 12/10 } \\
11 a\end{array}$ & 0 & $\begin{array}{l}M 7 / 11 \\
5 p\end{array}$ & 0 & $\begin{array}{l}\text { W 16/11 } \\
12.10 p\end{array}$ & 0 \\
\hline
\end{tabular}




\begin{tabular}{|c|c|c|c|c|c|c|c|c|c|c|c|c|c|c|c|c|c|c|}
\hline & Name & & Hui 1 & $\begin{array}{c}\text { Hui } 1 \\
\text { Date/ time }\end{array}$ & $\begin{array}{c}\mathrm{H} 1 \\
\mathrm{R}\end{array}$ & $\begin{array}{l}\text { Hui } 2 \\
\text { Date/ } \\
\text { time }\end{array}$ & $\begin{array}{c}\mathrm{H} 2 \\
\mathrm{R}\end{array}$ & $\begin{array}{l}\mathrm{FG} \\
\text { Date/ } \\
\text { time }\end{array}$ & $\begin{array}{c}\mathrm{FG} \\
\mathrm{R}\end{array}$ & $\begin{array}{l}\text { Hui } 3 \\
\text { Date/ } \\
\text { time }\end{array}$ & $\begin{array}{c}\mathrm{H} 3 \\
\mathrm{R}\end{array}$ & $\begin{array}{l}\mathrm{FG}(\mathrm{T}) \\
\text { Date/ } \\
\text { time }\end{array}$ & $\begin{array}{l}\text { Hui } 4 \\
\text { Date/ } \\
\text { time }\end{array}$ & $\begin{array}{c}\mathrm{H} 4 \\
\mathrm{R}\end{array}$ & $\begin{array}{l}\mathrm{FG} \\
\text { Date/ } \\
\text { time }\end{array}$ & FG R & $\begin{array}{c}\text { Ind } \\
\text { Int } \\
\text { Date/ } \\
\text { time }\end{array}$ & $\begin{array}{l}\text { II } \\
\mathrm{P}\end{array}$ \\
\hline \multirow[t]{4}{*}{ D } & Paul & $\mathrm{T}$ & $T$ & $\begin{array}{l}M 13 / 6 \\
12.20 p\end{array}$ & 0 & $\begin{array}{l}\text { W22/6 } \\
3 p\end{array}$ & 0 & $\begin{array}{l}M 8 / 8 \\
12.40 p\end{array}$ & 0 & M12/9 1.20p & $1 \mathrm{~b}$ & - & - & - & - & - & $\begin{array}{l}M \text { 14/11 } \\
11.20 a\end{array}$ & 0 \\
\hline & Piri & $S$ & $S$ & $M 13 / 63 p$ & 0 & $\begin{array}{l}\text { W22/6 } \\
3 p\end{array}$ & 0 & - & $\begin{array}{l}3 d, \\
a, a\end{array}$ & M12/9 1.20p & $1 e$ & - & $\begin{array}{l}\text { (not held due } \\
\text { to teacher } \\
\text { WD) }\end{array}$ & & Abs, b & & $\begin{array}{l}F \quad 18 / 111.20 p \\
\& \quad F \quad 25 / 11 \\
1.20 p\end{array}$ & 0 \\
\hline & Lorena & $\mathrm{P}$ & $P$ & $M 13 / 65 p$ & 0 & $\begin{array}{l}\text { W22/6 } \\
3 p\end{array}$ & 0 & abs & $2 b, d$ & WD & - & - & - & - & - & - & - & \\
\hline & Peter & $S$ & $S$ & $M 13 / 63 p$ & 0 & $\begin{array}{l}W 22 / 6 \\
3 p\end{array}$ & 0 & - & $\begin{array}{l}2 \mathrm{~d} \\
\mathrm{~b}, \mathrm{a}\end{array}$ & M12/9 1.20p & $1 e$ & - & $\begin{array}{l}\text { (not held due } \\
\text { to teacher } \\
\text { WD) }\end{array}$ & & Abs, $d$ & & $\begin{array}{l}F \quad 18 / 111.20 p \\
\& \quad F \quad 25 / 11 \\
1.20 p\end{array}$ & 0 \\
\hline \multirow[t]{4}{*}{$E$} & Tom & $T$ & $\mathrm{Tm}$ & $F 17 / 63 p$ & 0 & $\begin{array}{l}F 17 / 6 \\
3 p\end{array}$ & 0 & $\begin{array}{l}M 8 / 8 \\
12.40 p\end{array}$ & 0 & Fr $9 / 93 p$ & $1 \mathrm{e}$ & $\begin{array}{l}\text { Fr23/9 } \\
12.40 p\end{array}$ & $\begin{array}{l}F \quad 18 / 11 \\
3 p\end{array}$ & $1 f$ & Abs, b & & $\begin{array}{l}\text { Th } 17 / 11 \\
8.50 \mathrm{a}\end{array}$ & 0 \\
\hline & David & $S$ & $\mathrm{Tm}$ & $F 17 / 63 p$ & 0 & $\begin{array}{l}\mathrm{F} 17 / 6 \\
3 p\end{array}$ & 0 & $\begin{array}{l}\mathrm{F} 24 / 6 \\
3 p\end{array}$ & 0 & Fr $9 / 93 p$ & $1 e$ & - & $\begin{array}{l}\text { F 18/11 } \\
3 p\end{array}$ & $1 f$ & $\begin{array}{l}M 7 / 11 \\
3 p\end{array}$ & 0 & $\begin{array}{l}F 25 / 11 \\
3 p\end{array}$ & 0 \\
\hline & Shea & $S$ & $\mathrm{Tm}$ & $F 17 / 63 p$ & 0 & $\begin{array}{l}F 17 / 6 \\
3 p\end{array}$ & 0 & $\begin{array}{l}\mathrm{F} 24 / 6 \\
3 p\end{array}$ & 0 & Fr $9 / 93 p$ & $1 \mathrm{~b}$ & - & $\begin{array}{l}F 18 / 11 \\
3 p\end{array}$ & $1 f$ & Abs, b & & $\begin{array}{l}F 25 / 11 \\
3 p\end{array}$ & 0 \\
\hline & Toby & $S$ & $\mathrm{Tm}$ & $F 17 / 63 p$ & 0 & $\begin{array}{l}\mathrm{F} 17 / 6 \\
3 p\end{array}$ & 0 & $\begin{array}{l}\mathrm{F} 24 / 6 \\
3 p\end{array}$ & 0 & Fr $9 / 93 p$ & $1 b$ & - & $\begin{array}{l}F 18 / 11 \\
3 p\end{array}$ & $1 f$ & Abs, b & & $\begin{array}{l}F 25 / 11 \\
3 p\end{array}$ & 0 \\
\hline
\end{tabular}

Key:

S: Students

T: Teachers

Mp: Mixed pair

Mg: Mixed group

R: Reschedule (shows number of reschedules)

P: Parents

Tm: Team

\section{Reasons for reschedule:}

a sickness, absence from school

b another commitment eg tangi, work commitment, family commitment, sports training

forgot hui

d unknown

members

needed more time to complete agreed actions 
Appendix C: Broad themes in findings emerging from data analysis

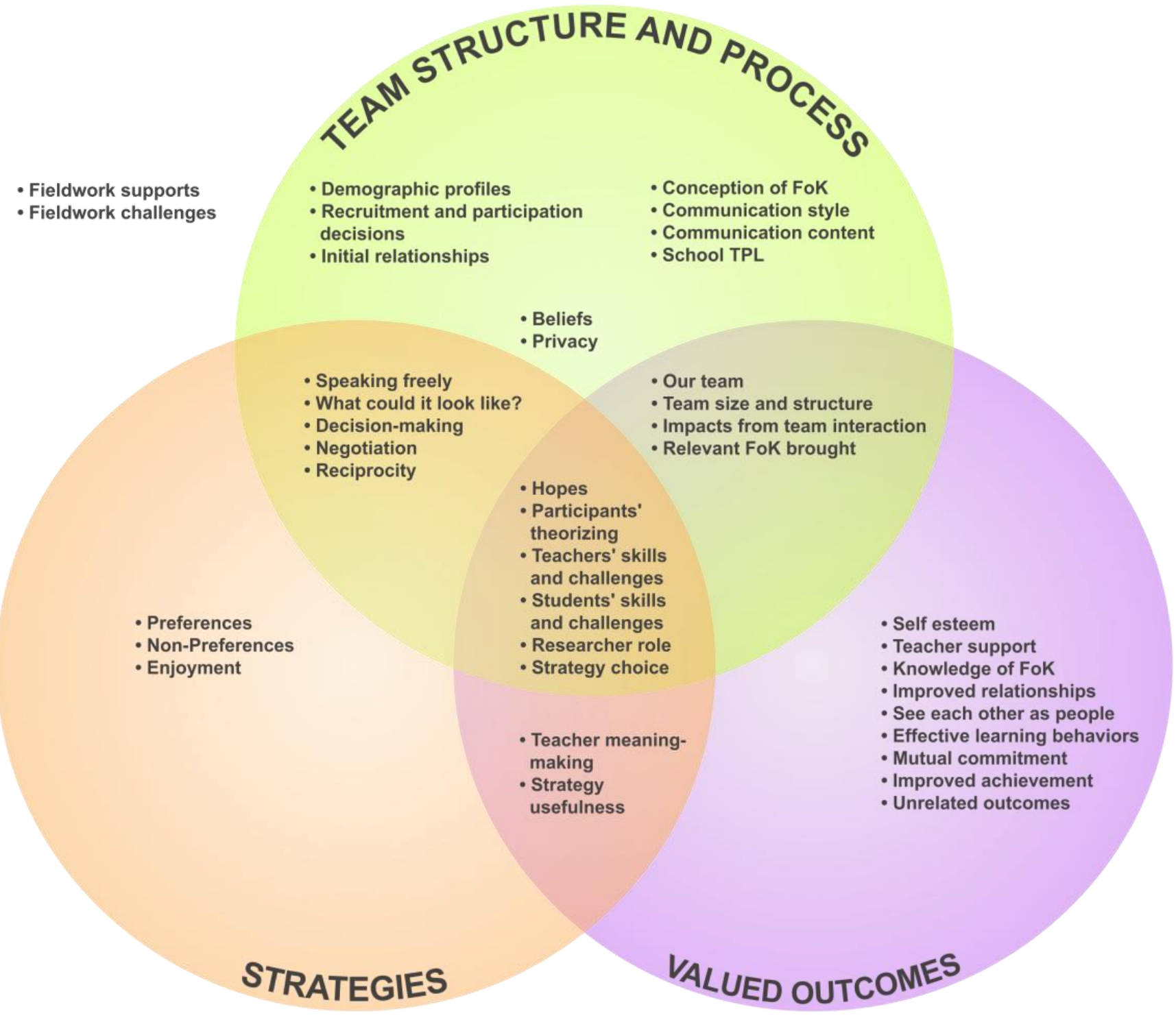

